TRANSACTIONS OF THE

AMERICAN MATHEMATICAL SOCIETY

Volume 365, Number 9, September 2013, Pages 4729-4809

S 0002-9947(2013)05832-1

Article electronically published on February 27, 2013

\title{
WEIGHTED LOCAL ORLICZ-HARDY SPACES ON DOMAINS AND THEIR APPLICATIONS IN INHOMOGENEOUS DIRICHLET AND NEUMANN PROBLEMS
}

\author{
JUN CAO, DER-CHEN CHANG, DACHUN YANG, AND SIBEI YANG
}

ABSTRACT. Let $\Omega$ be either $\mathbb{R}^{n}$ or a strongly Lipschitz domain of $\mathbb{R}^{n}$, and $\omega \in A_{\infty}\left(\mathbb{R}^{n}\right)$ (the class of Muckenhoupt weights). Let $L$ be a second-order divergence form elliptic operator on $L^{2}(\Omega)$ with the Dirichlet or Neumann boundary condition, and assume that the heat semigroup generated by $L$ has the Gaussian property $\left(G_{1}\right)$ with the regularity of their kernels measured by $\mu \in(0,1]$. Let $\Phi$ be a continuous, strictly increasing, subadditive, positive and concave function on $(0, \infty)$ of critical lower type index $p_{\Phi}^{-} \in(0,1]$. In this paper, the authors first introduce the "geometrical" weighted local OrliczHardy spaces $h_{\omega, r}^{\Phi}(\Omega)$ and $h_{\omega, z}^{\Phi}(\Omega)$ via the weighted local Orlicz-Hardy spaces $h_{\omega}^{\Phi}\left(\mathbb{R}^{n}\right)$, and obtain their two equivalent characterizations in terms of the nontangential maximal function and the Lusin area function associated with the heat semigroup generated by $L$ when $p_{\Phi}^{-} \in(n /(n+\mu), 1]$. Second, the authors furthermore establish three equivalent characterizations of $h_{\omega, r}^{\Phi}(\Omega)$ in terms of the grand maximal function, the radial maximal function and the atomic decomposition when the complement of $\Omega$ is unbounded and $p_{\Phi}^{-} \in$ $(0,1]$. Third, as applications, the authors prove that the operators $\nabla^{2} \mathbb{G}_{D}$ are bounded from $h_{\omega, r}^{\Phi}(\Omega)$ to the weighted Orlicz space $L_{\omega}^{\Phi}(\Omega)$, and from $h_{\omega, r}^{\Phi}(\Omega)$ to itself when $\Omega$ is a bounded semiconvex domain in $\mathbb{R}^{n}$ and $p_{\Phi}^{-} \in\left(\frac{n}{n+1}, 1\right]$, and the operators $\nabla^{2} \mathbb{G}_{N}$ are bounded from $h_{\omega, z}^{\Phi}(\Omega)$ to $L_{\omega}^{\Phi}(\Omega)$, and from $h_{\omega, z}^{\Phi}(\Omega)$ to $h_{\omega, r}^{\Phi}(\Omega)$ when $\Omega$ is a bounded convex domain in $\mathbb{R}^{n}$ and $p_{\Phi}^{-} \in\left(\frac{n}{n+1}, 1\right]$, where $\mathbb{G}_{D}$ and $\mathbb{G}_{N}$ denote, respectively, the Dirichlet Green operator and the Neumann Green operator.

\section{Contents}

1. Introduction

2. Preliminaries

2.1. Some properties of the weight class $A_{\infty}\left(\mathbb{R}^{n}\right) \quad 4741$

2.2. The divergence form elliptic operator $L \quad 4743$

2.3. Orlicz functions

Received by the editors July 25, 2011.

2010 Mathematics Subject Classification. Primary 42B35; Secondary 42B30, 42B20, 42B25, 35J25, 42B37, 47B38, 46E30.

Key words and phrases. Strongly Lipschitz domain, semiconvex domain, convex domain, divergence form elliptic operator, Dirichlet Green operator, Neumann Green operator, Dirichlet boundary condition, Neumann boundary condition, weight, local Orlicz-Hardy space, Gaussian property, nontangential maximal function, Lusin area function, atom.

The second author was partially supported by an NSF grant DMS-1203845 and a Hong Kong RGC competitive earmarked research grant \#601410.

The third (corresponding) author was supported by the National Natural Science Foundation of China (Grant No. 11171027) and the Specialized Research Fund for the Doctoral Program of Higher Education of China (Grant No. 20120003110003). 
3. Proof of Theorem 1.4

4. Proof of Theorem 1.7

5. Proof of Theorem 1.8

6. Proof of Theorem 1.9

Acknowledgements

References

\section{INTRODUCTION}

The theory of Hardy spaces on the $n$-dimensional Euclidean space $\mathbb{R}^{n}$ was originally initiated by Stein and Weiss in [77. Later, Fefferman and Stein [30] systematically developed a real-variable theory for the Hardy spaces $H^{p}\left(\mathbb{R}^{n}\right)$ with $p \in(0,1]$, which are designed to behave well under the Calderón-Zygmund operators; see, for example, [19, 67, 75, 76]. In particular, they respect translations, rotations, and dilations. However, there are two shortcomings of the spaces $H^{p}\left(\mathbb{R}^{n}\right)$. It is known that the spaces $H^{p}\left(\mathbb{R}^{n}\right)$ are not closed under compositions with diffeomorphisms nor under multiplication by smooth functions with compact support. In order to overcome these issues, Goldberg [37] developed the theory of the local Hardy spaces $h^{p}\left(\mathbb{R}^{n}\right)$ with $p \in(0,1]$, which plays an important role in partial differential equations and harmonic analysis; see, for example, [12, 37, 74, 78, and their references. In particular, one may prove that pseudo-differential operators of order zero are bounded on the spaces $h^{p}\left(\mathbb{R}^{n}\right)$ with $p \in(0,1]$; see [37. (also [79, 80]). In [12, Bui studied the weighted version $h_{\omega}^{p}\left(\mathbb{R}^{n}\right)$ of the local Hardy space $h^{p}\left(\mathbb{R}^{n}\right)$ with $\omega \in A_{\infty}\left(\mathbb{R}^{n}\right)$, where and in what follows, $A_{q}\left(\mathbb{R}^{n}\right)$ for $q \in[1, \infty]$ denotes the class of Muckenhoupt weights; see, for example, 33 . for their definitions and properties.

Rychkov 74] introduced a class of local weights, denoted by $A_{\infty}^{\text {loc }}\left(\mathbb{R}^{n}\right)$, and studied the weighted Besov-Lipschitz spaces and Triebel-Lizorkin spaces with weights belonging to $A_{\infty}^{\text {loc }}\left(\mathbb{R}^{n}\right)$, which contains $A_{\infty}\left(\mathbb{R}^{n}\right)$ weights as special cases. In particular, Rychkov [74] generalized some of the results of Bui [12] on weighted local Hardy spaces $h_{\omega}^{p}\left(\mathbb{R}^{n}\right)$ to $A_{\infty}^{\text {loc }}\left(\mathbb{R}^{n}\right)$ weights. Very recently, Tang 78 established the weighted atomic decomposition characterization of the weighted local Hardy space $h_{\omega}^{p}\left(\mathbb{R}^{n}\right)$ with $\omega \in A_{\infty}^{\text {loc }}\left(\mathbb{R}^{n}\right)$ via the local grand maximal function.

On the other hand, as a generalization of $L^{p}\left(\mathbb{R}^{n}\right)$, the Orlicz space was introduced by Birnbaum-Orlicz in [11] and Orlicz in 68. Since then, the theory of the Orlicz spaces themselves has been well developed and these spaces have been widely used in probability, statistics, potential theory, and partial differential equations, as well as harmonic analysis and some other fields of mathematics; see, for example, 13 . 45, 59, 70, 71. Moreover, Orlicz-Hardy spaces are also suitable substitutions of the Orlicz spaces in the study of boundedness of operators; see, for example, 46, 48, 49, 51, 82. Recall that Orlicz-Hardy spaces and their dual spaces were studied by Janson [46] on $\mathbb{R}^{n}$ and Viviani [82] on spaces of homogeneous type in the sense of Coifman and Weiss 21] (see also [22]). Let $\Phi$ be a continuous, strictly increasing, subadditive, positive and concave function on $(0, \infty)$ of strictly critical lower type index $p_{\Phi} \in(0,1]$ (see (2.13) below for the definition of $\left.p_{\Phi}\right)$. Based on the works of Rychkov [74] and Tang 78, the weighted local Orlicz-Hardy spaces $h_{\omega}^{\Phi}\left(\mathbb{R}^{n}\right)$ with $\omega \in A_{\infty}^{\text {loc }}\left(\mathbb{R}^{n}\right)$ were introduced and studied in [86]. We point out that the 
assumptions on $p_{\Phi}$ in [86] can be relaxed into the same assumptions on $p_{\Phi}^{-}$; see (2.12) below for the definition of $p_{\Phi}^{-}$and also Remark 2.7 below.

As we mentioned at the beginning, Hardy spaces $H^{p}\left(\mathbb{R}^{n}\right)$ are essentially related to the second-order elliptic operator with constant coefficients,

$$
L:=\sum_{j, k=1}^{n} a_{j k} \frac{\partial^{2}}{\partial x_{i}^{2}},
$$

where $\left\{a_{j k}\right\}_{j, k=1}^{n}$ are constants and $\left(a_{j k}\right)_{n \times n}>0$. In recent years, the research of the real-variable theory of various function spaces associated with different differential operators has inspired great interests; see, for example, 6, 17, 28, 29, 38, 39, 40, 41, 50, 85. Moreover, Orlicz-Hardy spaces associated with some differential operators and their dual spaces were introduced and studied in [48, 49, 51]. In particular, the local Hardy space $h_{L}^{1}\left(\mathbb{R}^{n}\right)$, associated with a linear operator $L$ in $L^{2}\left(\mathbb{R}^{n}\right)$ which generates an analytic semigroup with kernels satisfying an upper bound of Poisson type, was also studied in 52 .

Next, it is natural to develop a theory of Hardy spaces on domains of $\mathbb{R}^{n}$; see, for example, 8, 16, 17, 18, 27, 43, 44, 66, 81]. As we may expect, there are several ways to define Hardy spaces on domains. In particular, the second author of this paper, Krantz and Stein [18] introduced the Hardy spaces $H_{r}^{p}(\Omega)$ and $H_{z}^{p}(\Omega)$ on domains $\Omega$ of $\mathbb{R}^{n}$, respectively, by restricting arbitrary elements of $H^{p}\left(\mathbb{R}^{n}\right)$ to $\Omega$, and restricting elements of $H^{p}\left(\mathbb{R}^{n}\right)$ which are zero outside $\bar{\Omega}$ to $\Omega$, where and in what follows, $\bar{\Omega}$ denotes the closure of $\Omega$ in $\mathbb{R}^{n}$. For these Hardy spaces, atomic decompositions have been obtained in 18 , when $\Omega$ is a special Lipschitz domain or a bounded Lipschitz domain of $\mathbb{R}^{n}$. The second author of this paper, Krantz and Stein [18, also introduced the local Hardy spaces $h_{r}^{p}(\Omega)$ and $h_{z}^{p}(\Omega)$ in a similar way and obtained atomic decompositions for these local Hardy spaces when $\Omega$ is a special Lipschitz domain or a bounded Lipschitz domain of $\mathbb{R}^{n}$. Furthermore, the dual spaces of these Hardy spaces and local Hardy spaces were studied in [15]. Let $\Omega$ be a strongly Lipschitz domain and let $H_{r}^{1}(\Omega)$ and $H_{z}^{1}(\Omega)$ be defined as in [18. Auscher and Russ [8] proved that $H_{r}^{1}(\Omega)$ and $H_{z}^{1}(\Omega)$ are characterized by the nontangential maximal function and the Lusin area function associated with $\left\{e^{-t \sqrt{L}}\right\}_{t \geq 0}$, respectively, under the so-called Dirichlet and Neumann boundary conditions, where $L$ is a second-order divergence form elliptic operator such that for all $t \in(0, \infty)$, the kernel of $e^{-t L}$ has the Gaussian property $\left(G_{\infty}\right)$ (see, for example, 8, Definition 3] or Definition 2.4 below). Let $\Phi$ be a continuous, strictly increasing, subadditive, positive function on $(0, \infty)$ of strictly critical lower type index

$$
p_{\Phi} \in\left(\frac{n}{n+\mu}, 1\right] .
$$

The Orlicz-Hardy spaces $H_{r}^{\Phi}(\Omega)$ and $H_{z}^{\Phi}(\Omega)$ were first introduced, respectively, in [87] and [88. Similar to [86], we point out that the assumptions on $p_{\Phi}$ in [87, 88] can also be relaxed into the same assumptions on $p_{\Phi}^{-}$; see Remark 2.7 below.

Let $h_{r}^{1}(\Omega)$ and $h_{z}^{1}(\Omega)$ be defined as in [18. Auscher and Russ $[8$ ] also showed that $h_{r}^{1}(\Omega)$ and $h_{z}^{1}(\Omega)$ can be characterized by the local nontangential maximal function and the local Lusin area function associated with $\left\{e^{-t \sqrt{L}}\right\}_{t \geq 0}$, respectively, under the Dirichlet and the Neumann boundary conditions. Here $L$ is a second-order divergence form elliptic operator satisfying the Gaussian property $\left(G_{1}\right)$. 
Let $p \in(0,1]$ and $\Omega$ be a proper open subset of $\mathbb{R}^{n}$. Let $H_{r}^{p}(\Omega)$ be defined as in [18]. Miyachi [66 obtained three equivalent characterizations of $H_{r}^{p}(\Omega)$ in terms of the grand maximal function, the radial maximal function and the atomic decomposition. The dual theory of $H_{r}^{p}(\Omega)$ was also obtained in 66 . Let $\Omega$ be a bounded Lipschitz domain of $\mathbb{R}^{n}$. Wang and Yang 84 introduced the weighted local Hardy spaces on $\Omega$ by restricting arbitrary elements of $h_{\omega}^{p}\left(\mathbb{R}^{n}\right)$ to $\Omega$ with $\omega \in A_{\infty}\left(\mathbb{R}^{n}\right)$. They characterized the space $h_{\omega, r}^{p}(\Omega)$ in terms of the grand maximal function, the radial maximal function and the atomic decomposition. They also applied these characterizations to harmonic functions defined on bounded Lipschitz domains.

Let $\Omega$ be a domain of $\mathbb{R}^{n}$. In what follows, we denote by $W^{1,2}(\Omega)$ the usual Sobolev space on $\Omega$ equipped with the norm

$$
\left\{\|f\|_{L^{2}(\Omega)}^{2}+\|\nabla f\|_{L^{2}(\Omega)}^{2}\right\}^{1 / 2}
$$

where $\nabla f$ denotes the distributional gradient of $f$. In what follows, $W_{0}^{1,2}(\Omega)$ stands for the closure of $C_{c}^{\infty}(\Omega)$ in $W^{1,2}(\Omega)$, where $C_{c}^{\infty}(\Omega)$ denotes the set of all $C^{\infty}$ functions on $\Omega$ with compact support.

Now we recall the inhomogeneous Dirichlet problem and Neumann problem on bounded domains of $\mathbb{R}^{n}$. Given an open, bounded subset $\Omega$ of $\mathbb{R}^{n}$ and $f \in C^{\infty}(\bar{\Omega})$, we denote by $\mathbb{G}_{D}(f)$ the unique solution in $W_{0}^{1,2}(\Omega)$ of the inhomogeneous Dirichlet problem

$$
\begin{cases}\Delta u=f, & \text { in } \Omega, \\ u=0, & \text { on } \partial \Omega,\end{cases}
$$

and refer to $\mathbb{G}_{D}$ as the Dirichlet Green operator. Given an open, bounded subset $\Omega$ of $\mathbb{R}^{n}$ and $f \in C^{\infty}(\bar{\Omega})$, we denote by $\mathbb{G}_{N}(f)$ the unique solution in $W^{1,2}(\Omega)$ of the inhomogeneous Neumann problem

$$
\begin{cases}\Delta u=f, & \text { in } \Omega, \\ \partial_{\nu} u=0, & \text { on } \partial \Omega,\end{cases}
$$

where it is assumed that $\int_{\Omega} f(x) d x=0$ and the solution is normalized by requiring that $\int_{\Omega} u(x) d x=0, \nu(x)$ denotes the unit outward normal to $\partial \Omega$ at $x \in \partial \Omega$, and $\partial_{\nu}:=\nabla \cdot \nu$ stands for the normal derivative.

Let $\Omega$ be a bounded smooth domain in $\mathbb{R}^{n}$. The regularity of the operators $\mathbb{G}_{D}$ and $\mathbb{G}_{N}$ on $L^{p}(\Omega)$ spaces, for $p \in(1, \infty)$, is well known; see, for example, [4, 57. More results, including extensions to local Hardy spaces $h_{r}^{p}(\Omega)$ or $h_{z}^{p}(\Omega)$ for $p \in(0,1]$, were obtained in [16, 17, 18. A natural question is to study the regularity of these Green operators on $L^{p}$ spaces for $p \in(1, \infty)$ on a bounded domain $\Omega$ in $\mathbb{R}^{n}$ under weaker smoothness hypotheses on the boundary $\partial \Omega$ of $\Omega$. Similarly, one may ask whether the $L^{p}(\Omega)$ estimates can be replaced by local Hardy spaces, $h_{r}^{p}(\Omega)$ or $h_{z}^{p}(\Omega)$, for $p \in(0,1]$. We give a brief survey of the progress in this direction via (i) through (vi) as follows (see also [26]):

(i) One early result in this line of work obtained by Kadlec [54] is that the mappings

$$
f \mapsto \frac{\partial^{2} \mathbb{G}_{D}(f)}{\partial x_{i} \partial x_{j}}, i, j \in\{1, \cdots, n\},
$$


are well defined and bounded on $L^{2}(\Omega)$ whenever $\Omega$ is a bounded convex domain in $\mathbb{R}^{n}$; see also 1 , 35.

(ii) Assume that $\Omega$ is a bounded convex domain in $\mathbb{R}^{n}$. The mappings in (1.3) were shown to be of weak type $(1,1)$, independently, by Dahlberg et al. 25] and Fromm [31, and to be bounded on a suitable Hardy space by Adolfsson [2]. By interpolation, these mappings are bounded on $L^{p}(\Omega)$ for $p \in(1,2)$.

(iii) The $L^{2}$-boundedness of the mappings,

$$
f \mapsto \frac{\partial^{2} \mathbb{G}_{N}(f)}{\partial x_{i} \partial x_{j}}, i, j \in\{1, \cdots, n\},
$$

has been known since the mid 1970s (see, for example, [35]) when $\Omega$ is a bounded convex domain in $\mathbb{R}^{n}$, but the optimal $L^{p}(\Omega)$ estimates, valid in the range $p \in(1,2]$, have only been proved in 1994 by Adolfsson and Jerison [3]. Their method was to obtain an endpoint estimate for atoms in a suitable Hardy space $H^{1}(\Omega)$, and then to use interpolation with the $L^{2}(\Omega)$ results.

(iv) For $p \in(0,1]$, the regularity of the Green operators $\mathbb{G}_{D}$ and $\mathbb{G}_{N}$ on scales of local Hardy spaces, $h_{r}^{p}(\Omega)$ or $h_{z}^{p}(\Omega)$, have been studied by Mayboroda and Mitrea [60, 61] when $\Omega$ is a bounded Lipschitz domain in $\mathbb{R}^{n}$, and the results were formulated in terms of a pair of Hardy spaces, $h_{r}^{p}(\Omega)$ and $h_{z}^{p}(\Omega)$, for the range $p \in\left(\frac{n}{n+\epsilon}, 1\right)$ for some $\epsilon \in(0,1]$. Recently, Mitrea et al. 63] studied the mapping properties of the mappings in (1.3) on Besov and Triebel-Lizorkin spaces in a bounded Lipschitz domain satisfying a uniform exterior ball condition. In particular, the mappings in (1.3) are bounded from $h_{r}^{p}(\Omega)$ into itself when $p \in\left(\frac{n}{n+1}, 1\right]$.

(v) Very recently, Mitrea et al. [64] proved that these mappings in (1.3) and (1.4) are bounded on $L^{2}(\Omega)$ when $\Omega$ is a bounded semiconvex domain in $\mathbb{R}^{n}$ (which contains bounded convex domains). Moreover, Duong et al. 26] obtained the boundedness of these mappings in (1.3) from the local Hardy spaces $h_{\Delta_{D}}^{p}(\Omega)$ to $L^{p}(\Omega)$ for the range $p \in(0,1]$ and proved that these mappings are also of weak type $(1,1)$, when $\Omega$ is a bounded simply connected semiconvex domain in $\mathbb{R}^{n}$. By interpolation, these mappings are bounded on $L^{p}(\Omega)$ for all $p \in(1,2)$. In [26], Duong et al. also showed that these mappings in (1.4) are bounded from the local Hardy spaces $h_{\Delta_{N}}^{p}(\Omega)$ to $L^{p}(\Omega)$ for the range $p \in(0,1]$ and are of weak type $(1,1)$, when $\Omega$ is a bounded simply connected semiconvex domain in $\mathbb{R}^{n}$. Hence, by interpolation again, these mappings are bounded on $L^{p}(\Omega)$ for $p \in(1,2)$. Based on these results, Duong et al. [26] proved that the mappings in (1.3) are bounded on the local Hardy space $h_{r}^{p}(\Omega)$, for the range $p \in\left(\frac{n}{n+1}, 1\right]$, when $\Omega$ is a bounded simply connected semiconvex domain in $\mathbb{R}^{n}$; and the mappings in (1.4) are bounded from the space $h_{z}^{p}(\Omega)$ to the space $h_{r}^{p}(\Omega)$ when $p \in\left(\frac{n}{n+1}, 1\right]$ and $\Omega$ is a bounded simply connected convex domain in $\mathbb{R}^{n}$.

(vi) In relation to (ii) and (iii) above, it should be mentioned that the aforementioned $L^{p}(\Omega)$ boundedness of the mappings in (1.3) and (1.4) may fail in the class of Lipschitz domains $\Omega$ for any $p \in(1, \infty)$ and in the class of convex domains $\Omega$ for any $p \in(2, \infty)$ (see [2, 3, 24, 47] for counterexamples; recall that every convex domain is a Lipschitz domain).

Let $\Omega$ be either $\mathbb{R}^{n}$ or a strongly Lipschitz domain of $\mathbb{R}^{n}$, and $\omega \in A_{\infty}\left(\mathbb{R}^{n}\right)$. Let $L$ be a second-order divergence form elliptic operator on $L^{2}(\Omega)$ with the Dirichlet or Neumann boundary condition, and assume that the heat semigroup generated by $L$ has the Gaussian property $\left(G_{1}\right)$ with the regularity of their kernels measured 
by $\mu \in(0,1]$. Let $\Phi$ be a continuous, strictly increasing, subadditive, positive and concave function on $(0, \infty)$ of critical lower type index $p_{\Phi}^{-} \in(0,1]$. A typical example of such functions is

$$
\Phi(t):=t^{p} \quad \text { for all } \quad t \in(0, \infty) \text { and } p \in(0,1] .
$$

More examples are given in Section 2.3 below. First, motivated by $8,16,18,49$, 51, 86, we introduce the weighted local Orlicz-Hardy spaces $h_{\omega, r}^{\Phi}(\Omega)$ and $h_{\omega, z}^{\Phi}(\Omega)$, respectively, by restricting arbitrary elements of $h_{\omega}^{\Phi}\left(\mathbb{R}^{n}\right)$ to $\Omega$ or by restricting elements of $h_{\omega}^{\Phi}\left(\mathbb{R}^{n}\right)$, which are zero outside $\bar{\Omega}$, to $\Omega$, where $h_{\omega}^{\Phi}\left(\mathbb{R}^{n}\right)$ denotes the weighted local Orlicz-Hardy space introduced in 86 . Then we establish the atomic decompositions of these spaces by means of the Lusin area function associated with $\left\{e^{-t L}\right\}_{t \geq 0}$. Applying this, we obtain two equivalent characterizations of $h_{\omega, r}^{\Phi}(\Omega)$ and $h_{\omega, z}^{\Phi}(\Omega)$, respectively, in terms of the nontangential maximal function and the Lusin area function associated with $\left\{e^{-t L}\right\}_{t \geq 0}$. Second, motivated by [66, we furthermore establish three equivalent characterizations of the spaces $h_{\omega, r}^{\Phi}(\Omega)$, respectively, in terms of the grand maximal function, the radial maximal function and the atomic decomposition, when the complement of $\Omega$ is unbounded and $p_{\Phi}^{-} \in$ $(0,1]$. Third, as applications, we prove that the operators $\nabla^{2} \mathbb{G}_{D}$ are bounded from $h_{\omega, r}^{\Phi}(\Omega)$ to the weighted Orlicz space $L_{\omega}^{\Phi}(\Omega)$ and from $h_{\omega, r}^{\Phi}(\Omega)$ to itself, when $\Omega$ is a bounded semiconvex domain in $\mathbb{R}^{n}$ and $p_{\Phi}^{-} \in\left(\frac{n}{n+1}, 1\right]$, and the operators $\nabla^{2} \mathbb{G}_{N}$ are bounded from $h_{\omega, z}^{\Phi}(\Omega)$ to $L_{\omega}^{\Phi}(\Omega)$ and from $h_{\omega, z}^{\Phi}(\Omega)$ to $h_{\omega, r}^{\Phi}(\Omega)$, when $\Omega$ is a bounded convex domain in $\mathbb{R}^{n}$ and $p_{\Phi}^{-} \in\left(\frac{n}{n+1}, 1\right]$, where $\mathbb{G}_{D}$ and $\mathbb{G}_{N}$ denote, respectively, the Dirichlet Green operator and the Neumann Green operator.

To state the main results of this paper, we first recall some necessary notions. Throughout the whole paper, we always assume that $\Omega$ is a strongly Lipschitz domain of $\mathbb{R}^{n}$; namely, $\Omega$ is a proper open connected set in $\mathbb{R}^{n}$ whose boundary is a finite union of parts of rotated graphs of Lipschitz maps, at most one of these parts possibly unbounded. It is well known that strongly Lipschitz domains include special Lipschitz domains, bounded Lipschitz domains and exterior domains; see, for example, 8, 10] for their definitions and properties.

Also, throughout the entire paper, for the sake of convenience, we choose the norm on $\mathbb{R}^{n}$ to be the supremum norm; namely, for any $x=\left(x_{1}, x_{2}, \cdots, x_{n}\right) \in \mathbb{R}^{n}$,

$$
|x|:=\max \left\{\left|x_{1}\right|, \cdots,\left|x_{n}\right|\right\},
$$

for which balls determined by this norm are cubes associated with the usual Euclidean norm with sides parallel to the axes.

Remark 1.1. Let $\Omega$ be a strongly Lipschitz domain of $\mathbb{R}^{n}$. Then $\Omega$ is a space of homogeneous type in the sense of Coifman and Weiss [21. Furthermore, as a space of homogeneous type, the collection of all balls of $\Omega$ is given by the set

$$
\left\{Q \cap \Omega \text { : cube } Q \subset \mathbb{R}^{n} \text { satisfying } x_{Q} \in \Omega \text { and } l(Q) \leq 2 \operatorname{diam}(\Omega)\right\},
$$

where $x_{Q}$ denotes the center of $Q, l(Q)$ the sidelength of $Q$ and $\operatorname{diam}(\Omega)$ the diameter of $\Omega$, namely,

$$
\operatorname{diam}(\Omega):=\sup \{|x-y|: x, y \in \Omega\} ;
$$

see, for example, [8].

To introduce the spaces $h_{\omega, r}^{\Phi}(\Omega)$ and $h_{\omega, z}^{\Phi}(\Omega)$, we first recall the definition of the weighted local Orlicz-Hardy space $h_{\omega}^{\Phi}\left(\mathbb{R}^{n}\right)$ introduced in 86. Let $\mathcal{D}\left(\mathbb{R}^{n}\right)$ denote 
the space of all infinitely differentiable functions with compact support in $\mathbb{R}^{n}$ endowed with the inductive topology, and $\mathcal{D}^{\prime}\left(\mathbb{R}^{n}\right)$ its topological dual with the weak-* topology which is called the space of distributions on $\mathbb{R}^{n}$. For all $f \in \mathcal{D}^{\prime}\left(\mathbb{R}^{n}\right)$, let $\mathcal{G}^{\text {loc }}(f)$ denote its local grand maximal function; see [86, Definition 3.1].

Definition 1.2. Let $\Phi$ satisfy Assumption (A) (see Section 2.2 for the definition of Assumption (A)) and $\omega \in A_{\infty}\left(\mathbb{R}^{n}\right)$ (see Definition 2.1 below for the definition of $\left.A_{\infty}\left(\mathbb{R}^{n}\right)\right)$. Define

$$
h_{\omega}^{\Phi}\left(\mathbb{R}^{n}\right):=\left\{f \in \mathcal{D}^{\prime}\left(\mathbb{R}^{n}\right): \int_{\mathbb{R}^{n}} \Phi\left(\mathcal{G}^{\text {loc }}(f)(x)\right) \omega(x) d x<\infty\right\}
$$

and

$$
\|f\|_{h_{\omega}^{\Phi}\left(\mathbb{R}^{n}\right)}:=\inf \left\{\lambda \in(0, \infty): \int_{\mathbb{R}^{n}} \Phi\left(\frac{\mathcal{G}^{\text {loc }}(f)(x)}{\lambda}\right) \omega(x) d x \leq 1\right\} .
$$

In what follows, let $\mathcal{D}(\Omega)$ denote the space of all infinitely differentiable functions with compact support in $\Omega$ endowed with the inductive topology, and $\mathcal{D}^{\prime}(\Omega)$ its topological dual with the weak-* topology which is called the space of distributions on $\Omega$.

Definition 1.3. Let $\Phi$ and $\omega$ be as in Definition 1.2 and $\Omega$ a subdomain of $\mathbb{R}^{n}$. A distribution $f$ on $\Omega$ is said to be in the weighted local Orlicz-Hardy space $h_{\omega, r}^{\Phi}(\Omega)$ if $f$ is the restriction to $\Omega$ of a distribution $F$ in $h_{\omega}^{\Phi}\left(\mathbb{R}^{n}\right)$; namely,

$$
\begin{aligned}
h_{\omega, r}^{\Phi}(\Omega): & =\left\{f \in \mathcal{D}^{\prime}(\Omega): \text { there exists an } F \in h_{\omega}^{\Phi}\left(\mathbb{R}^{n}\right) \text { such that }\left.F\right|_{\Omega}=f\right\} \\
& =h_{\omega}^{\Phi}\left(\mathbb{R}^{n}\right) /\left\{F \in h_{\omega}^{\Phi}\left(\mathbb{R}^{n}\right): F=0 \text { on } \Omega\right\} .
\end{aligned}
$$

Moreover, for all $f \in h_{\omega, r}^{\Phi}(\Omega)$, the norm of $f$ in $h_{\omega, r}^{\Phi}(\Omega)$ is defined by

$$
\|f\|_{h_{\omega, r}^{\Phi}(\Omega)}:=\inf \left\{\|F\|_{h_{\omega}^{\Phi}(\Omega)}: F \in h_{\omega}^{\Phi}\left(\mathbb{R}^{n}\right) \text { and }\left.F\right|_{\Omega}=f\right\},
$$

where the infimum is taken over all $F \in h_{\omega}^{\Phi}\left(\mathbb{R}^{n}\right)$ satisfying $F=f$ on $\Omega$.

The weighted local Orlicz-Hardy space $h_{\omega, z}^{\Phi}(\Omega)$ is defined by

$$
h_{\omega, z}^{\Phi}(\Omega):=\left\{f \in h_{\omega}^{\Phi}\left(\mathbb{R}^{n}\right): f=0 \text { on }(\bar{\Omega})^{\complement}\right\} /\left\{f \in h_{\omega}^{\Phi}\left(\mathbb{R}^{n}\right): f=0 \text { on } \Omega\right\},
$$

where $(\bar{\Omega})^{\complement}$ denotes the set $\mathbb{R}^{n} \backslash \bar{\Omega}$. Moreover, for any $f \in h_{\omega, z}^{\Phi}(\Omega)$, its norm in $h_{\omega, z}^{\Phi}(\Omega)$ is defined by

$$
\|f\|_{h_{\omega, z}^{\Phi}(\Omega)}:=\inf \left\{\|F\|_{h_{\omega}^{\Phi}\left(\mathbb{R}^{n}\right)}: F \in h_{\omega}^{\Phi}\left(\mathbb{R}^{n}\right), F=0 \text { on }(\bar{\Omega})^{\complement} \text { and }\left.F\right|_{\Omega}=f\right\} .
$$

Let $\Omega$ be either $\mathbb{R}^{n}$ or a strongly Lipschitz domain of $\mathbb{R}^{n}$. Let $\Phi$ satisfy Assumption (A), $\omega \in A_{\infty}\left(\mathbb{R}^{n}\right)$ and $L$ be a divergence form elliptic operator on $L^{2}(\Omega)$ with the Dirichlet boundary condition (for simplicity, DBC) or the Neumann boundary condition (for simplicity, NBC) (see (2.7) below for the definition of $L$ and Definition 2.3 below for DBC and NBC). Let the spaces $h_{\mathcal{N}_{h}, \omega}^{\Phi}(\Omega), h_{\widetilde{S}_{h}, \omega}^{\Phi}(\Omega)$ and $h_{S_{h}, \omega}^{\Phi}(\Omega)$ be respectively as in Definitions 3.1 and 3.3 below. Then one of the main results of this paper is as follows.

Theorem 1.4. Let $\Phi$ satisfy Assumption (A), $\omega \in A_{\infty}\left(\mathbb{R}^{n}\right)$ and $L$ be as in (2.7). Let $q_{\omega}, r_{\omega}, \mu, p_{\Phi}^{+}$and $p_{\Phi}^{-}$be respectively as in (2.5), (2.6), (2.9), (2.11) and (2.12). 
Let $\Omega$ be either $\mathbb{R}^{n}$ or a strongly Lipschitz domain of $\mathbb{R}^{n}$. Assume that $q_{\omega}, r_{\omega}, \mu$, $p_{\Phi}^{+}$and $p_{\Phi}^{-}$satisfy the inequalities $\frac{q_{\omega}}{p_{\Phi}^{-}}<\frac{n+\mu}{n}$,

$$
\frac{2 q_{\omega}}{p_{\Phi}^{-}}<\frac{n+1}{n}+\frac{r_{\omega}-1}{p_{\Phi}^{+} r_{\omega}}
$$

and $r_{\omega}>\frac{2}{2-q_{\omega}}$, and the semigroup generated by $L$ has the Gaussian property $\left(G_{1}\right)$.

(i) If $\Omega:=\mathbb{R}^{n}$, then the spaces

$$
h_{\omega}^{\Phi}\left(\mathbb{R}^{n}\right), h_{\mathcal{N}_{h}, \omega}^{\Phi}\left(\mathbb{R}^{n}\right), h_{\widetilde{S}_{h}, \omega}^{\Phi}\left(\mathbb{R}^{n}\right) \text { and } h_{S_{h}, \omega}^{\Phi}\left(\mathbb{R}^{n}\right)
$$

coincide with equivalent quasi-norms.

(ii) Under $D B C$, if $\Omega^{\complement}$ is unbounded, then the spaces

$$
h_{\omega, r}^{\Phi}(\Omega), h_{\mathcal{N}_{h}, \omega}^{\Phi}(\Omega), h_{\widetilde{S}_{h}, \omega}^{\Phi}(\Omega) \text { and } h_{S_{h}, \omega}^{\Phi}(\Omega)
$$

coincide with equivalent quasi-norms.

(iii) Under $N B C$, the spaces

$$
h_{\omega, z}^{\Phi}(\Omega), h_{\mathcal{N}_{h}, \omega}^{\Phi}(\Omega), h_{\widetilde{S}_{h}, \omega}^{\Phi}(\Omega) \text { and } h_{S_{h}, \omega}^{\Phi}(\Omega)
$$

coincide with equivalent quasi-norms.

We first point out that the coincidence between $h_{\omega}^{\Phi}\left(\mathbb{R}^{n}\right)$ and $h_{\mathcal{N}_{h}, \omega}^{\Phi}\left(\mathbb{R}^{n}\right)$, or between $h_{\omega, r}^{\Phi}(\Omega)$ and $h_{\mathcal{N}_{h}, \omega}^{\Phi}(\Omega)$, or between $h_{\omega, z}^{\Phi}(\Omega)$ and $h_{\mathcal{N}_{h}, \omega}^{\Phi}(\Omega)$ of Theorem 1.4 when $\Phi(t):=t$ for all $t \in(0, \infty)$ and $\omega \equiv 1$ was already obtained by Auscher and Russ in [8, Theorems 2 and 20].

The proofs of (i) through (iii) of Theorem 1.4 are similar and can be given by following the main approach used by Auscher and Russ in [8] with some subtle modifications. Here we only give the framework of the proof of Theorem 1.4(ii). The following chains of inequalities give the strategy of the proof of Theorem 1.4(ii). For all $f \in h_{\omega, r}^{\Phi}(\Omega) \cap L^{2}(\Omega)$ and any given $R_{0} \in\left[\frac{1}{2}, \infty\right)$, we have

$$
\begin{aligned}
\|f\|_{h_{\omega}^{\Phi}, r}(\Omega) & \gtrsim\left\|\mathcal{N}_{h}^{\text {loc }, 2 R_{0}}(f)\right\|_{L_{\omega}^{\Phi}(\Omega)} \\
& \gtrsim\left\|\widetilde{S}_{h, R_{0}}^{\text {loc }}(f)\right\|_{L_{\omega}^{\Phi}(\Omega)}+\mathrm{I}(f) \\
& \gtrsim\left\|S_{h, R_{0}}^{\text {loc }}(f)\right\|_{L_{\omega}^{\Phi}(\Omega)}+\mathrm{I}(f) \gtrsim\|f\|_{h_{\omega, r}^{\Phi}(\Omega)},
\end{aligned}
$$

where the implicit constants are independent of $f$, and $\mathcal{N}_{h}^{\text {loc, } 2 R_{0}}(f), \widetilde{S}_{h, R_{0}}^{\text {loc }}(f)$ and $S_{h, R_{0}}^{\text {loc }}(f)$ are respectively as in Definitions 3.1 and 3.3 below, and

$$
\mathrm{I}(f):=\inf \left\{\lambda \in(0, \infty): \sum_{\widetilde{Q}_{k} \in \mathcal{Q}_{\Omega}} \omega\left(\widetilde{Q}_{k} \cap \Omega\right) \Phi\left(\frac{m_{\widetilde{Q}_{k} \cap \Omega}\left(\left|e^{-R_{0}^{2} L}(f)\right|\right)}{\lambda}\right) \leq 1\right\}
$$

with $m_{\widetilde{Q}_{k} \cap \Omega}\left(\left|e^{-R_{0}^{2} L}(f)\right|\right)$ being the average of $\left|e^{-R_{0}^{2} L}(f)\right|$ on $\widetilde{Q}_{k} \cap \Omega$ (see (3.1) below). Moreover, see Section 3 for the definitions of $\mathcal{Q}_{\Omega}$ and $\widetilde{Q}_{k}$. Theorem 1.4 (ii) is deduced from (1.5) and the arbitrariness of $R_{0} \in\left[\frac{1}{2}, \infty\right)$. The proof of the first inequality in (1.5) is standard by applying the atomic decomposition of $h_{\omega}^{\Phi}\left(\mathbb{R}^{n}\right)$ obtained in [86] and the relation between $h_{\omega, r}^{\Phi}(\Omega)$ and $h_{\omega}^{\Phi}\left(\mathbb{R}^{n}\right)$; see Proposition 3.4 below. We prove the second and the third inequalities, respectively, in Propositions 3.8 and 3.12 below. We point out that Proposition 3.8 plays an important role in the proof of Theorem 1.4(ii), and the key step in the proof of Proposition 3.8 
is to establish a "good- $\lambda$ inequality" concerning $\mathcal{N}_{h}^{\text {loc, } 2 R_{0}}(f)$ and $\widetilde{S}_{h, R_{0}}^{\text {loc }}(f)$ (see Lemma 3.10 below), which is a subtle weighted variant on the local nontangential maximal function and the local Lusin-area function of [8, Lemma 9] and even if its special case that $\omega \equiv 1$ also improves [8, Lemma 9] in the sense that [8, Lemma $9]$ presents a "good- $\lambda$ inequality" on the nontangential maximal function and the average of the Lusin-area function, not the Lusin-area function itself (see also [88]). To show the last inequality of (1.5) in Proposition 3.13(i) below, for all $f \in L^{2}(\Omega)$ satisfying $\left\|S_{h, R_{0}}^{\text {loc }}(f)\right\|_{L_{\omega}^{\Phi}(\Omega)}<\infty$, we establish its atomic decomposition by using a local Calderón reproducing formula (see (3.39) below) on $L^{2}(\Omega)$ associated with $L$, the atomic decomposition of functions in the tent space on $\Omega$, and the reflection technology related to Lipschitz domains on $\mathbb{R}^{n}$ which was proved by Auscher and Russ in [8, p. 183] and plays a key role in the proof of Theorem 1.4 (see also Lemma 3.15 below). But, this reflection technology was not necessary in the proof of Theorem 1.4(iii) (see also [8]). We point out that the method used in the proof of the last inequality of (1.5) is also quite different from that used in the proof of [8, Proposition 19] for a similar inequality on $h_{r}^{1}(\Omega)$. Since $h_{\omega, r}^{\Phi}(\Omega)$ when $p_{\Phi}^{-}<1$ is only a quasi-Banach space, not a Banach space, and the norms of elements in quasi-Banach spaces cannot be achieved by duality, the method used in the proof of [8, Proposition 19] cannot be applied to the current situation; see also [88.

Let $p \in\left(\frac{n}{n+1}, 1\right]$. Recall that bounded convex domains and semiconvex domains in $\mathbb{R}^{n}$ are strongly Lipschitz domains of $\mathbb{R}^{n}$. We remark that when $L=-\Delta$ with the Dirichlet boundary condition, $\Omega$ is a bounded semiconvex domain in $\mathbb{R}^{n}, \omega \equiv 1$ and $\Phi(t):=t^{p}$ for all $t \in(0, \infty)$, Theorem 1.4(ii) coincides with [26, Proposition 5.3(ii)]; when $L=-\Delta$ with the Neumann boundary condition, $\Omega$ is a bounded convex domain in $\mathbb{R}^{n}, \omega \equiv 1$ and $\Phi(t):=t^{p}$ for all $t \in(0, \infty)$, Theorem 1.4(iii) was also obtained in [26, Proposition 5.3(i)]. We remark that the approach used in the proofs of [26. Theorem 3.5 and Proposition 5.3] is quite different from that used in the proof of Theorem 1.4. A key tool used in the proofs of [26, Theorem 3.5 and Proposition 5.3] is the atomic characterization closely associated with $-\Delta$ on domains, while in the proof of Theorem 1.4, we fully use the atomic characterization of $h_{\omega}^{\Phi}\left(\mathbb{R}^{n}\right)$ in [86].

To state the second main theorem of this paper, we first recall the following notions of the radial and the grand maximal functions on $\Omega$.

Definition 1.5. Let $\Omega$ be an open subset of $\mathbb{R}^{n}$. Denote the boundary of $\Omega$ by $\partial \Omega$. For all $x \in \Omega$, let $\delta(x):=\operatorname{dist}(x, \partial \Omega)$. Let

$$
\varphi \in \mathcal{D}\left(\mathbb{R}^{n}\right) \text { with } \operatorname{supp}(\varphi) \subset B(0,1) \text { and } \int_{\mathbb{R}^{n}} \varphi(x) d x=1 .
$$

Let $c_{0} \in(1, \infty)$. For any $f \in \mathcal{D}^{\prime}(\Omega)$, the radial maximal function $f_{\varphi, \Omega}^{+}$of $f$ associated to $\varphi$ on $\Omega$ is defined by setting, for all $x \in \Omega$,

$$
f_{\varphi, \Omega}^{+}(x):=\sup _{0<t<\delta(x) / c_{0}}\left|\varphi_{t} * f(x)\right|,
$$

where and in what follows, for all $t \in(0, \infty)$ and $x \in \mathbb{R}^{n}$,

$$
\varphi_{t}(x):=\frac{1}{t^{n}} \varphi\left(\frac{x}{t}\right) .
$$


The grand maximal function $f_{\Omega}^{*}$ of $f$ on $\Omega$ is defined by setting, for all $x \in \Omega$,

$$
f_{\Omega}^{*}(x):=\sup _{0<t<\delta(x) / c_{0}} \sup _{\psi \in \mathcal{F}_{t}(x)}|\langle f, \psi\rangle|,
$$

where for all $x \in \Omega$,

$$
\begin{aligned}
\mathcal{F}_{t}(x):=\left\{\psi \in \mathcal{D}\left(\mathbb{R}^{n}\right): \operatorname{supp}(\psi) \subset B(x, t),\right. \\
\left.\qquad \sup _{y \in \mathbb{R}^{n}}\left|\partial^{\alpha} \psi(y)\right| \leq t^{-|\alpha|-n} \text { for every } \alpha \in \mathbb{Z}_{+}^{n}\right\} .
\end{aligned}
$$

We also need the following notions of local $(\rho, q, s)_{\omega}$-atoms on $\Omega$. Recall that the space $L_{\omega}^{q}(\Omega)$, for $q \in[1, \infty]$ and $\omega \in A_{\infty}\left(\mathbb{R}^{n}\right)$, denotes the weighted Lebesgue space endowed with the norm that, for any $f \in L_{\omega}^{q}(\Omega)$, when $q \in[1, \infty)$,

and

$$
\|f\|_{L_{\omega}^{q}(\Omega)}:=\left\{\int_{\Omega}|f(x)|^{q} \omega(x) d x\right\}^{1 / q},
$$

$$
\|f\|_{L_{\omega}^{\infty}(\omega)}:=\|f\|_{L^{\infty}(\Omega)} .
$$

Definition 1.6. Let $\Omega$ be an open subset of $\mathbb{R}^{n}$ and $\omega \in A_{\infty}\left(\mathbb{R}^{n}\right), q_{\omega}, p_{\Phi}^{-}$and $\rho$ be respectively as in (2.5), (2.12) and (2.14) below. A triplet $(\rho, q, s)_{\omega}$ is called admissible if $q \in\left(q_{\omega}, \infty\right]$ and $s \in \mathbb{Z}_{+}$with $s \geq\left\lfloor n\left(\frac{q_{\omega}}{p_{\Phi}^{-}}-1\right)\right\rfloor$. A function $a$ supported on a cube $Q \subset \Omega$ is called a type (a) local $(\rho, q, s)_{\omega}$-atom if $4 Q \cap \partial \Omega=\emptyset$ with $l(Q)<1$,

and

$$
\|a\|_{L_{\omega}^{q}\left(\mathbb{R}^{n}\right)} \leq[\omega(Q)]^{\frac{1}{q}-1}[\rho(\omega(Q))]^{-1}
$$

$$
\int_{\mathbb{R}^{n}} a(x) x^{\alpha} d x=0
$$

for all $\alpha \in \mathbb{Z}_{+}^{n}$ with $|\alpha| \leq s$.

A function $a$ supported on a cube $Q \subset \Omega$ is called a type (b) local $(\rho, q, s)_{\omega}$-atom if either $l(Q) \geq 1$ or $2 Q \cap \partial \Omega=\emptyset$ and $4 Q \cap \partial \Omega \neq \emptyset$, and

$$
\|a\|_{L_{\omega}^{q}\left(\mathbb{R}^{n}\right)} \leq[\omega(Q)]^{\frac{1}{q}-1}[\rho(\omega(Q))]^{-1} .
$$

Theorem 1.7. Let $\Phi$ satisfy Assumption (A), $\omega \in A_{\infty}\left(\mathbb{R}^{n}\right), \Omega$ be a strongly Lipschitz domain of $\mathbb{R}^{n}$ satisfying that $\Omega^{\complement}$ is unbounded, and $\varphi$ be as in (1.6). Then the following are equivalent:

(i) $f \in h_{\omega, r}^{\Phi}(\Omega)$;

(ii) $f \in \mathcal{D}^{\prime}(\Omega)$ and $f_{\varphi, \Omega}^{+} \in L_{\omega}^{\Phi}(\Omega)$, where $f_{\varphi, \Omega}^{+}$is as in (1.7);

(iii) $f \in \mathcal{D}^{\prime}(\Omega)$ and $f_{\Omega}^{*} \in L_{\omega}^{\Phi}(\Omega)$, where $f_{\Omega}^{*}$ is as in (1.8);

(iv) $f \in \mathcal{D}^{\prime}(\Omega)$ and

$$
f=\sum_{\text {type }(a) \text { atoms }} \lambda_{Q} a_{Q}+\sum_{\text {type }(b) \text { atoms }} \mu_{Q} b_{Q}
$$

in $\mathcal{D}^{\prime}(\Omega)$, where $(\rho, q, s)_{\omega}$ is an admissible triplet, $\left\{a_{Q}\right\}_{Q}$ is a sequence of type $(a)$ local $(\rho, q, s)_{\omega}$-atoms, $\left\{b_{Q}\right\}_{Q}$ is a sequence of type (b) local $(\rho, q, s)_{\omega}$-atoms and $\left\{\lambda_{Q}\right\}_{Q} \cup\left\{\mu_{Q}\right\}_{Q} \subset \mathbb{C}$ satisfying

$$
\sum_{\text {type (a) atoms }} \omega(Q) \Phi\left(\frac{\left|\lambda_{Q}\right|}{\omega(Q) \rho(\omega(Q))}\right)
$$




$$
+\sum_{\text {type (b) atoms }} \omega(Q) \Phi\left(\frac{\left|\lambda_{Q}\right|}{\omega(Q) \rho(\omega(Q))}\right)<\infty
$$

Moreover, define

$$
\begin{array}{r}
\Lambda\left(\left\{\lambda_{Q} a_{Q}\right\}_{Q} \cup\left\{\mu_{Q} b_{Q}\right\}_{Q}\right) \\
:=\inf \left\{\lambda \in(0, \infty): \sum_{\text {type (a) atoms }} \omega(Q) \Phi\left(\frac{\left|\lambda_{Q}\right|}{\lambda \omega(Q) \rho(\omega(Q))}\right)\right. \\
\left.\quad+\sum_{\text {type (b) atoms }} \omega(Q) \Phi\left(\frac{\left|\lambda_{Q}\right|}{\lambda \omega(Q) \rho(\omega(Q))}\right) \leq 1\right\}
\end{array}
$$

and

$$
\|f\|_{h_{\omega}^{\rho, q, s}(\Omega)}:=\inf \left\{\Lambda\left(\left\{\lambda_{Q} a_{Q}\right\}_{Q} \cup\left\{\mu_{Q} b_{Q}\right\}_{Q}\right)\right\},
$$

where the infimum is taken over all the decompositions of $f$ as above.

Furthermore, for all $f \in h_{\omega, r}^{\Phi}(\Omega)$,

$$
\|f\|_{h_{\omega, r}^{\Phi}(\Omega)} \sim\left\|f_{\varphi, \Omega}^{+}\right\|_{L_{\omega}^{\Phi}(\Omega)} \sim\left\|f_{\Omega}^{*}\right\|_{L_{\omega}^{\Phi}(\Omega)} \sim\|f\|_{h_{\omega}^{\rho, q, s}(\Omega)},
$$

where the implicit constants are independent of $f$.

The following outline gives the strategy of the proof of Theorem 1.7 (i) $\Longleftrightarrow$ (iv) $\Longrightarrow$ (iii) $\Longleftrightarrow$ (ii) $\Longrightarrow$ (i). We divide the proof of Theorem 1.7 into the following four steps. Step I: (i) $\Longleftrightarrow$ (iv); Step II: (iii) $\Longleftrightarrow$ (ii); Step III: (iv) $\Longrightarrow$ (iii); Step IV: (ii) $\Longrightarrow$ (i). In Step I, we prove that (i) implies (iv) by using the atomic characterization of $h_{\omega}^{\Phi}\left(\mathbb{R}^{n}\right)$ and the definition of the space $h_{\omega, r}^{\Phi}(\Omega)$; moreover, for any given $f \in \mathcal{D}^{\prime}(\Omega)$ satisfying (iv) of Theorem 1.7 we construct an $F \in h_{\omega}^{\Phi}\left(\mathbb{R}^{n}\right)$ such that $\left.F\right|_{\Omega}=f$ and

$$
\|F\|_{h_{\omega}^{\Phi}\left(\mathbb{R}^{n}\right)} \lesssim\|f\|_{h_{\omega}^{\rho, q, s}(\Omega)}
$$

by using the reflection technology related to Lipschitz domains on $\mathbb{R}^{n}$ from 8 ] (see also Lemma 3.15 below), which completes the proof that (iv) implies (i). In Step II, it is obvious that (iii) implies (ii); by using a useful estimate concerning $f_{\Omega, \varphi}^{+}$and $f_{\Omega}^{*}$, which was established by Miyachi [65] (see also (4.5) below), we prove that (ii) implies (iii). The proof of Step III is standard, which is similar to that of Proposition 3.4(i) below. In Step IV, for any given $f \in \mathcal{D}^{\prime}(\Omega)$ satisfying $f_{\varphi, \Omega}^{+} \in L_{\omega}^{\Phi}(\Omega)$, we construct an $F \in h_{\omega}^{\Phi}\left(\mathbb{R}^{n}\right)$ such that $\left.F\right|_{\Omega}=f$ by using two useful estimates established by Miyachi 66 (see also (4.7) and (4.8) below), which shows that (ii) implies (i). We remark that except for its own interest, Theorem 1.7 is also needed to obtain Theorems 1.8 and 1.9 below.

Theorem 1.8. Let $\Phi, \omega, q_{\omega}, r_{\omega}$ and $p_{\Phi}^{-}$be as in Theorem 1.4. Let $\Omega$ be a bounded, simply connected, semiconvex domain in $\mathbb{R}^{n}$, and $\mathbb{G}_{D}$ the Dirichlet Green operator for the problem (1.1). Then

(i) the operators in (1.3), originally defined on $C^{\infty}(\bar{\Omega})$, can be extended to bounded operators from $h_{\omega, r}^{\Phi}(\Omega)$ to $L_{\omega}^{\Phi}(\Omega)$;

(ii) the operators in (1.3), originally defined on $C^{\infty}(\bar{\Omega})$, can be extended to bounded operators from $h_{\omega, r}^{\Phi}(\Omega)$ to $h_{\omega, r}^{\Phi}(\Omega)$. 
The proof of Theorem 1.8 below follows the approach used in the proofs of Duong et al. 26, Theorems 4.1 and 5.4]. We prove Theorem 1.8(i) by using the atomic characterization of $h_{\omega, r}^{\Phi}(\Omega)$ obtained in Theorem 1.7, some useful estimates about the integral kernel of the Dirichlet Green operator $\mathbb{G}_{D}$ from Fromm [31] and Grüter-Widman 36] (see also Lemma 5.2 below), and the $L^{p}(\Omega)$-boundedness of the mappings in (1.3) from Duong et al. [26, Theorem 4.1] (see also Lemma 5.3 below). Furthermore, we prove Theorem 1.8(ii) by using Theorem 1.8 (i), the radial maximal function characterization of $h_{\omega, r}^{\Phi}(\Omega)$ obtained in Theorem 1.7 and the reflection technology related to Lipschitz domains on $\mathbb{R}^{n}$ from 8 .

Let $p \in(n /(n+1), 1]$. We point out that Theorem [1.8 (i) when $\Phi(t):=t^{p}$ for all $t \in(0, \infty)$ and $\omega \equiv 1$ was obtained in [26. Theorem 4.1]; and Theorem 1.8(ii) completely covers [26, Theorem 5.4] by taking $\Phi(t):=t^{p}$ for all $t \in(0, \infty)$ and $\omega \equiv 1$.

Theorem 1.9. Let $\Phi, \omega, q_{\omega}, r_{\omega}$ and $p_{\Phi}^{-}$be as in Theorem 1.4. Let $\Omega$ be a bounded, simply connected, convex domain in $\mathbb{R}^{n}$. Let $\mathbb{G}_{N}$ be the Neumann Green operator for the problem (1.2). Then

(i) the operators in (1.4), originally defined on

$$
\left\{f \in C^{\infty}(\bar{\Omega}): \int_{\Omega} f(x) d x=0\right\},
$$

can be extended to bounded operators from $h_{\omega, z}^{\Phi}(\Omega)$ to $L_{\omega}^{\Phi}(\Omega)$;

(ii) the operators in (1.4), originally defined on

$$
\left\{f \in C^{\infty}(\bar{\Omega}): \int_{\Omega} f(x) d x=0\right\}
$$

can be extended to bounded operators from $h_{\omega, z}^{\Phi}(\Omega)$ to $h_{\omega, r}^{\Phi}(\Omega)$.

Because the integral kernel of the Neumann Green operator $\mathbb{G}_{N}$ and its gradients do not have good enough size estimates which are similar to those in Lemma 5.3 , the method used in the proof of Theorem 1.8 is not valid in the proof of Theorem 1.9. Motivated by Duong et al. [26, Theorem 3.5 and Proposition 4.11], we establish a new atomic decomposition characterization of $h_{\omega, z}^{\Phi}(\Omega)$ via a class of atoms associated with the operator $L$, where $L$ denotes the Laplace operator on $L^{2}(\Omega)$ with the Neumann boundary condition; see Lemma 6.3 below. By using this auxiliary result and some integral estimates of the kernel $\left\{K_{t}\right\}_{t \geq 0}$ of the semigroup $\left\{e^{-t L}\right\}_{t \geq 0}$ (see Lemma 6.4 below), and the $L^{p}(\Omega)$-boundedness of the mappings in (1.4) from Duong et al. [26, Theorem 4.2] (see also Lemma 6.5] below), we prove Theorem 1.9(i). Furthermore, from the new atomic decomposition characterization of $h_{\omega, z}^{\Phi}(\Omega)$, Theorem 1.9 (i) and the radial maximal function characterization of $h_{\omega, r}^{\Phi}(\Omega)$ obtained in Theorem 1.7, we deduce Theorem 1.9](ii).

Let $p \in\left(\frac{n}{n+1}, 1\right]$. Theorem $1.9(\mathrm{i})$ when $\Phi(t):=t^{p}$ for all $t \in(0, \infty)$ and $\omega \equiv 1$ was obtained in [26, Theorem 4.2 and Proposition 5.3(i)]; also Theorem 1.9)(ii) completely covers [26, Theorem 5.7] by taking $\Phi(t):=t^{p}$ for all $t \in(0, \infty)$ and $\omega \equiv 1$.

By [74, we know that the local weight class $A_{\infty}^{\text {loc }}\left(\mathbb{R}^{n}\right)$ satisfies the exponential growth in the whole space $\mathbb{R}^{n}$, which implies that when $\omega \in A_{\infty}^{\text {loc }}\left(\mathbb{R}^{n}\right)$ but $\omega \notin$ $A_{\infty}\left(\mathbb{R}^{n}\right)$, (3.9) and (3.10) in the proof of Proposition 3.4 and (3.20) in the proof of Proposition 3.8 may not be true, respectively, due to the lack of the compact 
support of the kernel of $e^{-t^{2} L}$ or the exponential growth of $\omega$. Thus, throughout the whole paper, we always assume that $\omega \in A_{\infty}\left(\mathbb{R}^{n}\right)$.

The layout of this paper is as follows. In Section 2, we first recall the definition of the weight class $A_{\infty}\left(\mathbb{R}^{n}\right)$ and some of its properties; then we recall some properties of the divergence form elliptic operator $L$ on $\mathbb{R}^{n}$ or a strongly Lipschitz domain $\Omega$, and then describe some basic assumptions on $L$; finally we describe some basic assumptions on Orlicz functions and present some properties of these functions. In Section 3, we give the proof of Theorem 1.4. Section 4 is devoted to the proof of Theorem 1.7. In Sections 5 and [6] we respectively give the proofs of Theorems 1.8 and 1.9 .

Finally we make some conventions on notation. Throughout the entire paper, $L$ always denotes the second-order divergence form elliptic operator as in (2.7). We denote by $C$ a positive constant which is independent of the main parameters, but it may vary from line to line. We also use $C(\gamma, \beta, \cdots)$ to denote a positive constant depending on the indicated parameters $\gamma, \beta, \cdots$. The symbol $A \lesssim B$ means that $A \leq C B$. If $A \lesssim B$ and $B \lesssim A$, then we write $A \sim B$. The symbol $\lfloor s\rfloor$ for $s \in \mathbb{R}$ denotes the maximal integer not more than $s ; Q(x, t)$ denotes a closed cube in $\mathbb{R}^{n}$ with center $x \in \mathbb{R}^{n}$ and sidelength $l(Q):=t$ and $C Q(x, t):=Q(x, C t)$. For any given normed spaces $\mathcal{A}$ and $\mathcal{B}$ with the corresponding norms $\|\cdot\|_{\mathcal{A}}$ and $\|\cdot\|_{\mathcal{B}}, \mathcal{A} \subset \mathcal{B}$ means that for all $f \in \mathcal{A}$, then $f \in \mathcal{B}$ and $\|f\|_{\mathcal{B}} \lesssim\|f\|_{\mathcal{A}}$. For any subset $G$ of $\mathbb{R}^{n}$, we denote by $G^{\complement}$ the set $\mathbb{R}^{n} \backslash G$, and by $\chi_{G}$ its characteristic function. We also set $\mathbb{N}:=\{1,2, \cdots\}$ and $\mathbb{Z}_{+}:=\mathbb{N} \cup\{0\}$. For any $\theta:=\left(\theta_{1}, \ldots, \theta_{n}\right) \in \mathbb{Z}_{+}^{n}$, let

$$
|\theta|:=\theta_{1}+\cdots+\theta_{n} \quad \text { and } \quad \partial_{x}^{\theta}:=\frac{\partial^{|\theta|}}{\partial x_{1}^{\theta_{1}} \cdots \partial x_{n}^{\theta_{n}}} .
$$

For any sets $E, F \subset \mathbb{R}^{n}$ and $z \in \mathbb{R}^{n}$, let

$$
\operatorname{dist}(E, F):=\inf _{x \in E, y \in F}|x-y| \text { and } \operatorname{dist}(z, E):=\inf _{x \in E}|x-z| .
$$

\section{Preliminaries}

In Subsection 2.1, we first recall some properties of the class of Muckenhoupt weights; in Subsection 2.2, we then recall some properties of the divergence form elliptic operator $L$ on $\mathbb{R}^{n}$ or a strongly Lipschitz domain $\Omega$, and describe some basic assumptions on $L$; in Subsection 2.3. we describe some basic assumptions of Orlicz functions and then present some properties of these functions.

2.1. Some properties of the weight class $A_{\infty}\left(\mathbb{R}^{n}\right)$. In this subsection, we first recall the definitions and some properties of the class of Muckenhoupt weights and the reverse Hölder class; see, for example, [32, 33, 34.

Definition 2.1. (i) Let $p \in(1, \infty)$. The weight class $A_{p}\left(\mathbb{R}^{n}\right)$ is defined to be the set of all nonnegative locally integrable functions $\omega$ on $\mathbb{R}^{n}$ such that

$$
A_{p}(\omega):=\sup _{Q \subset \mathbb{R}^{n}} \frac{1}{|Q|^{p}} \int_{Q} \omega(x) d x\left(\int_{Q}[\omega(x)]^{-\frac{p^{\prime}}{p}} d x\right)^{\frac{p}{p^{\prime}}}<\infty,
$$

where the supremum is taken over all cubes $Q \subset \mathbb{R}^{n}$ and $\frac{1}{p}+\frac{1}{p^{\prime}}=1$. 
When $p=1$, the weight class $A_{1}\left(\mathbb{R}^{n}\right)$ is defined to be the set of all nonnegative locally integrable functions $\omega$ on $\mathbb{R}^{n}$ such that

$$
A_{1}(\omega):=\sup _{Q \subset \mathbb{R}^{n}} \frac{1}{|Q|} \int_{Q} \omega(x) d x\left(\underset{y \in Q}{\operatorname{ess} \sup }[\omega(y)]^{-1}\right)<\infty,
$$

where the supremum is taken over all cubes $Q \subset \mathbb{R}^{n}$.

(ii) Let $r \in(1, \infty)$. The reverse Hölder class $R H_{r}\left(\mathbb{R}^{n}\right)$ is defined to be the set of all nonnegative locally integrable functions $\omega$ on $\mathbb{R}^{n}$ such that

$$
R H_{r}(\omega):=\sup _{Q \subset \mathbb{R}^{n}}\left(\frac{1}{|Q|} \int_{Q}[\omega(x)]^{r} d x\right)^{1 / r}\left[\frac{1}{|Q|} \int_{Q} \omega(x) d x\right]^{-1}<\infty,
$$

where the supremum is taken over all cubes $Q \subset \mathbb{R}^{n}$.

When $r=\infty$, the reverse Hölder class $R H_{\infty}\left(\mathbb{R}^{n}\right)$ is defined to be the set of all nonnegative locally integrable functions $\omega$ on $\mathbb{R}^{n}$ such that

$$
R H_{\infty}(\omega):=\sup _{Q \subset \mathbb{R}^{n}}\left[\operatorname{essup}_{y \in Q} \omega(y)\right]\left[\frac{1}{|Q|} \int_{Q} \omega(x) d x\right]^{-1}<\infty,
$$

where the supremum is taken over all cubes $Q \subset \mathbb{R}^{n}$.

In what follows, for a Lebesgue measurable set $E \subset \mathbb{R}^{n}$ and a weight $\omega \in$ $A_{\infty}\left(\mathbb{R}^{n}\right)$, let

$$
\omega(E):=\int_{E} \omega(x) d x .
$$

Then we recall some properties of the Muckenhoupt weights as follows.

Lemma 2.2. (i) If $1 \leq p_{1}<p_{2}<\infty$, then $A_{p_{1}}\left(\mathbb{R}^{n}\right) \subset A_{p_{2}}\left(\mathbb{R}^{n}\right)$.

(ii) If $\omega \in A_{p}\left(\mathbb{R}^{n}\right)$ with $p \in(1, \infty)$, then there exists $q \in(1, p)$ such that $\omega \in$ $A_{q}\left(\mathbb{R}^{n}\right)$.

(iii) If $\omega \in A_{p}\left(\mathbb{R}^{n}\right)$ with $p \in[1, \infty)$, then there exists a positive constant $C$ such that for any cube $Q \subset \mathbb{R}^{n}$ and any measurable subset $E$ of $Q$,

$$
\frac{\omega(Q)}{\omega(E)} \leq C\left[\frac{|Q|}{|E|}\right]^{p}
$$

(iv) If $1<p_{1} \leq p_{2}<\infty$, then

$$
R H_{\infty}\left(\mathbb{R}^{n}\right) \subset R H_{p_{2}}\left(\mathbb{R}^{n}\right) \subset R H_{p_{1}}\left(\mathbb{R}^{n}\right) .
$$

(v) If $\omega \in R H_{r}\left(\mathbb{R}^{n}\right)$ with $r \in(1, \infty]$, then there exists a positive constant $C$ such that for any cube $Q \subset \mathbb{R}^{n}$ and any measurable subset $E$ of $Q$,

$$
\frac{\omega(E)}{\omega(Q)} \leq C\left[\frac{|E|}{|Q|}\right]^{\frac{r-1}{r}}
$$

(vi)

$$
\bigcup_{1 \leq p<\infty} A_{p}\left(\mathbb{R}^{n}\right)=\bigcup_{1<q \leq \infty} R H_{q}\left(\mathbb{R}^{n}\right) .
$$

(vii) Let $\omega \in A_{p}\left(\mathbb{R}^{n}\right)$ with $p \in(1, \infty), R_{0} \in(0, \infty)$ and $\mathcal{N}_{h}^{\text {loc, } R_{0}}$ be as in Definition 3.1 below. Then $\mathcal{N}_{h}^{\text {loc, } R_{0}}$ is bounded on $L_{\omega}^{p}\left(\mathbb{R}^{n}\right)$. 
Proof. The statements (i) through (vi) of this lemma and their proofs can be found in [33, 34. We omit the details. Now we prove Lemma 2.2(vii). Denote by $M$ the Hardy-Littlewood maximal operator on $\mathbb{R}^{n}$. Let $p \in(1, \infty)$ and $\omega \in A_{p}\left(\mathbb{R}^{n}\right)$. From (2.8) below, we deduce that

$$
\mathcal{N}_{h}^{\text {loc, } R_{0}}(f) \lesssim M(f)
$$

for all $f \in L_{\omega}^{p}\left(\mathbb{R}^{n}\right)$. By this and the well-known fact that $M$ is bounded on $L_{\omega}^{p}\left(\mathbb{R}^{n}\right)$, we know that $\mathcal{N}_{h}^{\text {loc, } R_{0}}$ is bounded on $L_{\omega}^{p}\left(\mathbb{R}^{n}\right)$, which completes the proof of Lemma 2.2 (vii) and hence Lemma 2.2 .

Let

$$
A_{\infty}\left(\mathbb{R}^{n}\right):=\bigcup_{1 \leq p<\infty} A_{p}\left(\mathbb{R}^{n}\right)=\bigcup_{1<q \leq \infty} R H_{q}\left(\mathbb{R}^{n}\right) .
$$

For any given $\omega \in A_{\infty}\left(\mathbb{R}^{n}\right)$, define the critical indexes $q_{\omega}$ and $r_{\omega}$ of $\omega$, respectively, by

$$
q_{\omega}:=\inf \left\{p \in[1, \infty): \omega \in A_{p}\left(\mathbb{R}^{n}\right)\right\}
$$

and

$$
r_{\omega}:=\sup \left\{r \in(1, \infty]: \omega \in R H_{r}\left(\mathbb{R}^{n}\right)\right\} .
$$

Recall that if $q_{\omega} \in(1, \infty)$, then $\omega \notin A_{q_{\omega}}\left(\mathbb{R}^{n}\right)$, and there exists $\omega \notin A_{1}\left(\mathbb{R}^{n}\right)$ such that $q_{\omega}=1$ (see [53]). Similarly, if $r_{\omega} \in(1, \infty)$, then $\omega \notin R H_{r_{\omega}}\left(\mathbb{R}^{n}\right)$, and there exists $\omega \notin R H_{\infty}\left(\mathbb{R}^{n}\right)$ such that $r_{\omega}=\infty$ (see $[23$ ).

2.2. The divergence form elliptic operator $L$. In this subsection, we describe the divergence form elliptic operators considered in this paper and the most typical example is the Laplace operator on the Lipschitz domain of $\mathbb{R}^{n}$ with the Dirichlet boundary condition or the Neumann boundary condition.

For $A: \mathbb{R}^{n} \rightarrow M_{n}(\mathbb{C})$ a measurable function, where $M_{n}(\mathbb{C})$ denotes the set of all $n \times n$ complex-valued matrixes, let

$$
\|A\|_{\infty}:=\operatorname{essiup}_{\substack{x \in \mathbb{R}^{n}, \xi, \eta \in \mathbb{C}^{n},|\xi|=|\eta|=1}}|A(x) \xi \cdot \bar{\eta}|
$$

where $\bar{\eta}$ denotes the conjugate vector of $\eta$. For all $\delta \in(0,1]$, denote by $\mathcal{A}(\delta)$ the class of all measurable functions $A: \mathbb{R}^{n} \rightarrow M_{n}(\mathbb{C})$ satisfying the ellipticity condition; namely, for all $x \in \mathbb{R}^{n}$ and $\xi \in \mathbb{C}^{n}$,

$$
\|A\|_{\infty} \leq \delta^{-1} \text { and } \Re(A(x) \xi \cdot \bar{\xi}) \geq \delta|\xi|^{2},
$$

where and in what follows, $\Re(A(x) \xi \cdot \bar{\xi})$ denotes the real part of $A(x) \xi \cdot \bar{\xi}$. Denote by $\mathcal{A}$ the union of all $\mathcal{A}(\delta)$ for $\delta \in(0,1]$.

When $A \in \mathcal{A}$ and $V$ is a closed subspace of $W^{1,2}(\Omega)$ containing $W_{0}^{1,2}(\Omega)$, denote by $L$ the maximal-accretive operator (see [69, p. 23, Definition 1.46] for the definition) on $L^{2}(\Omega)$ with largest domain $D(L) \subset V$ such that for all $f \in D(L)$ and $g \in V$,

$$
\langle L f, g\rangle=\int_{\Omega} A(x) \nabla f(x) \cdot \overline{\nabla g(x)} d x,
$$

where $\langle\cdot, \cdot\rangle$ denotes the interior product in $L^{2}(\Omega)$. In this sense, for all $f \in D(L)$, we write

$$
L f:=-\operatorname{div}(A \nabla f) .
$$


We recall the following Dirichlet and Neumann boundary conditions of $L$ from [8, p. 152].

Definition 2.3. Let $\Omega$ be either $\mathbb{R}^{n}$ or a strongly Lipschitz domain of $\mathbb{R}^{n}$ and $L$ be as in (2.7). The operator $L$ is said to satisfy the Dirichlet boundary condition (for simplicity, DBC) if $V:=W_{0}^{1,2}(\Omega)$, and the Neumann boundary condition (for simplicity, NBC) if $V:=W^{1,2}(\Omega)$.

Recall that if $\Omega:=\mathbb{R}^{n}$, then

$$
W_{0}^{1,2}(\Omega)=W^{1,2}(\Omega) .
$$

Thus, in this case, DBC and NBC are identical.

Let $L$ be as in (2.7). Then $L$ generates a semigroup $\left\{e^{-t L}\right\}_{t \geq 0}$ of operators that is analytic (namely, it has a holomorphic extension to a complex half cone $z \neq 0$ and $|\arg z|<\mu$ for some $\mu \in(0, \pi / 2)$ ) and contracting on $L^{2}(\Omega)$ (namely, for all $f \in L^{2}(\Omega)$ and $\left.t \in(0, \infty),\left\|e^{-t L} f\right\|_{L^{2}(\Omega)} \leq\|f\|_{L^{2}(\Omega)}\right)$; see, for example, [69, Proposition 1.51 and Theorem 1.52] for the details. Also, $L$ has a unique maximal accretive square root $\sqrt{L}$ such that $-\sqrt{L}$ generates an analytic and $L^{2}(\Omega)$ contracting semigroup $\left\{P_{t}\right\}_{t \geq 0}$ with $P_{t}:=e^{-t \sqrt{L}}$, the Poisson semigroup for $L$; see, for example, 55, for the details.

Now we recall the Gaussian property of $\left\{e^{-t L}\right\}_{t \geq 0}$ on a strongly Lipschitz domain; see, for example, [8, Definition 3] and also [9, 10].

Definition 2.4. Let $\Omega$ be either $\mathbb{R}^{n}$ or a strongly Lipschitz domain of $\mathbb{R}^{n}$, and let $L$ be as in (2.7). Let $\beta \in(0, \infty]$. The semigroup generated by $L$ is said to have the Gaussian property $\left(G_{\beta}\right)$ if the following (i) and (ii) hold:

(i) The kernel of $e^{-t L}$, denoted by $K_{t}$, is a measurable function on $\Omega \times \Omega$ and there exist positive constants $C$ and $\alpha$ such that for all $t \in(0, \beta)$ and all $x, y \in \Omega$,

$$
\left|K_{t}(x, y)\right| \leq \frac{C}{t^{n / 2}} e^{-\alpha \frac{|x-y|^{2}}{t}} .
$$

(ii) For all $x \in \Omega$ and $t \in(0, \beta)$, the functions $y \mapsto K_{t}(x, y)$ and $y \mapsto K_{t}(y, x)$ are Hölder continuous in $\Omega$ and there exist positive constants $C$ and $\mu \in(0,1]$ such that for all $t \in(0, \beta)$ and $x, y_{1}, y_{2} \in \Omega$,

$$
\left|K_{t}\left(x, y_{1}\right)-K_{t}\left(x, y_{2}\right)\right|+\left|K_{t}\left(y_{1}, x\right)-K_{t}\left(y_{2}, x\right)\right| \leq \frac{C}{t^{n / 2}} \frac{\left|y_{1}-y_{2}\right|^{\mu}}{t^{\mu / 2}} .
$$

Remark 2.5. (i) The assumption $\left(G_{\infty}\right)$ is always satisfied if $L$ is the Laplacian or a real operator (under DBC or NBC) on $\mathbb{R}^{n}$ or on Lipschitz domains except under NBC with $\Omega$ bounded; see, for example, [10.

(ii) The assumption $\left(G_{\infty}\right)$ implies that for all $\beta \in(0, \infty),\left(G_{\beta}\right)$ holds. If $\beta$ is finite, by [8, p. 178, Lemma A.1] and the property of semigroups, we know that $\left(G_{\beta}\right)$ and $\left(G_{1}\right)$ are equivalent.

The following well-known fact is a simple corollary of the analyticity of the semigroup $\left\{e^{-t L}\right\}_{t \geq 0}$. We omit the details.

Lemma 2.6. Let $\beta \in(0, \infty]$. Assume that $L$ has the Gaussian property $\left(G_{\beta}\right)$. Then the estimate (2.8) also holds for $t \partial_{t} K_{t}$. 
2.3. Orlicz functions. Let $\Phi$ be a positive function on $\mathbb{R}_{+}:=(0, \infty)$. The function $\Phi$ is said to be of upper type $p$ (resp. lower type $p$ ) for some $p \in[0, \infty)$ if there exists a positive constant $C$ such that for all $t \in[1, \infty)$ (resp. $t \in(0,1])$ and $s \in(0, \infty)$,

$$
\Phi(s t) \leq C t^{p} \Phi(s) .
$$

Obviously, if $\Phi$ is of lower type $p$ for some $p \in(0, \infty)$, then $\lim _{t \rightarrow 0^{+}} \Phi(t)=0$. So, for the sake of convenience, if it is necessary, we may assume that $\Phi(0)=0$. If $\Phi$ is of both upper type $p_{1}$ and lower type $p_{0}$, then $\Phi$ is said to be of type $\left(p_{0}, p_{1}\right)$. Let

$$
\begin{aligned}
p_{\Phi}^{+}:=\inf \{p \in(0, \infty): \text { there exists } C \in(0, \infty) \\
\text { such that (2.10) holds for all } t \in[1, \infty) \text { and } s \in(0, \infty)\}
\end{aligned}
$$

and

$$
\begin{aligned}
p_{\Phi}^{-}:=\sup \{p & \in(0, \infty): \text { there exists } C \in(0, \infty) \\
& \text { such that (2.10) holds for all } t \in(0,1] \text { and } s \in(0, \infty)\} .
\end{aligned}
$$

The function $\Phi$ is said to be of strictly lower type $p$ if for all $t \in(0,1)$ and $s \in(0, \infty), \Phi(s t) \leq t^{p} \Phi(s)$, and we define

$$
\begin{aligned}
p_{\Phi}:=\sup \{p \in(0, \infty): \Phi(s t) \leq & t^{p} \Phi(s) \text { holds } \\
& \quad \text { for all } t \in(0,1) \text { and } s \in(0, \infty)\} .
\end{aligned}
$$

It is easy to see that

$$
p_{\Phi} \leq p_{\Phi}^{-} \leq p_{\Phi}^{+}
$$

for all $\Phi$. In what follows, $p_{\Phi}, p_{\Phi}^{-}$and $p_{\Phi}^{+}$are respectively called the strictly critical lower type index, the critical lower type index and the critical upper type index of $\Phi$. Moreover, it was proved in [49, Remark 2.1] that $\Phi$ is also of strictly lower type $p_{\Phi}$. In other words, $p_{\Phi}$ is attainable.

Throughout the entire paper, we always assume that $\Phi$ satisfies the following assumptions.

Assumption (A). Let $\Phi$ be a positive function defined on $\mathbb{R}_{+}$which is of critical lower type $p_{\Phi}^{-} \in(0,1]$. Also assume that $\Phi$ is continuous, strictly increasing, subadditive and concave.

Remark 2.7. We observe that, via the Aoki-Rolewicz theorem in [5, 72, all results in $\left[86,87,88\right.$, are still true if the assumptions on $p_{\Phi}$ therein are relaxed into the same assumptions on $p_{\Phi}^{-}$.

It is worth noticing that $p_{\Phi}^{-}$and $p_{\Phi}^{+}$may not be attainable. For example, for $p \in(0,1]$, if $\Phi(t):=t^{p}$ for all $t \in(0, \infty)$, then $\Phi$ satisfies Assumption (A) and $p_{\Phi}=p_{\Phi}^{-}=p_{\Phi}^{+}=p$; for $p \in\left[\frac{1}{2}, 1\right]$, if

$$
\Phi(t):=t^{p} / \ln (e+t)
$$

for all $t \in(0, \infty)$, then $\Phi$ satisfies Assumption (A) and $p_{\Phi}^{-}=p=p_{\Phi}^{+}, p_{\Phi}^{-}$is not attainable, but $p_{\Phi}^{+}$is attainable; for $p \in\left(0, \frac{1}{2}\right]$, if

$$
\Phi(t):=t^{p} \ln (e+t)
$$

for all $t \in(0, \infty)$, then $\Phi$ satisfies Assumption $(\mathrm{A})$ and $p_{\Phi}^{-}=p=p_{\Phi}^{+}, p_{\Phi}^{-}$is attainable, but $p_{\Phi}^{+}$is not attainable. 
Notice that if $\Phi$ satisfies Assumption (A), then $\Phi(0)=0$ and $\Phi$ is obviously of upper type 1 . For any concave and positive function $\widetilde{\Phi}$ of lower type $p$, if we set

$$
\Phi(t):=\int_{0}^{t} \frac{\widetilde{\Phi}(s)}{s} d s
$$

for $t \in[0, \infty)$, then by [82, Proposition 3.1], $\Phi$ is equivalent to $\widetilde{\Phi}$; namely, there exists a positive constant $C$ such that

$$
C^{-1} \widetilde{\Phi}(t) \leq \Phi(t) \leq C \widetilde{\Phi}(t)
$$

for all $t \in[0, \infty)$. Moreover, $\Phi$ is a strictly increasing, concave, subadditive and continuous function of lower type $p$. Notice that all our results are invariant on equivalent functions satisfying Assumption (A). From this, we deduce that all results in this paper with $\Phi$ as in Assumption (A) also hold for all concave and positive functions $\widetilde{\Phi}$ of the same critical lower type $p_{\Phi}^{-}$as $\Phi$.

Since $\Phi$ is strictly increasing, we can define the function $\rho(t)$ on $\mathbb{R}_{+}$by setting, for all $t \in(0, \infty)$,

$$
\rho(t):=\frac{t^{-1}}{\Phi^{-1}\left(t^{-1}\right)}
$$

where $\Phi^{-1}$ is the inverse function of $\Phi$. Then the types of $\Phi$ and $\rho$ have the following relation: If $0<p_{0} \leq p_{1} \leq 1$ and $\Phi$ is an increasing function, then $\Phi$ is of type $\left(p_{0}, p_{1}\right)$ if and only if $\rho$ is of type $\left(p_{1}^{-1}-1, p_{0}^{-1}-1\right)$; see [82] for its proof.

\section{Proof of Theorem 1.4}

In this section, we first introduce the Orlicz-Hardy spaces

$$
h_{\mathcal{N}_{h}, \omega}^{\Phi}(\Omega), h_{S_{h}, \omega}^{\Phi}(\Omega) \text { and } h_{\widetilde{S}_{h}, \omega}^{\Phi}(\Omega),
$$

and then give the proof of Theorem 1.4 .

Let $\Omega$ be either $\mathbb{R}^{n}$ or a strongly Lipschitz domain of $\mathbb{R}^{n}$ and $\omega \in A_{\infty}\left(\mathbb{R}^{n}\right)$. Recall that for an Orlicz function $\Phi$ on $(0, \infty)$, a measurable function $f$ on $\Omega$ is said to be in the space $L_{\omega}^{\Phi}(\Omega)$ if

$$
\int_{\Omega} \Phi(|f(x)|) \omega(x) d x<\infty .
$$

Moreover, for any $f \in L_{\omega}^{\Phi}(\Omega)$, define its quasi-norm by

$$
\|f\|_{L_{\omega}^{\Phi}(\Omega)}:=\inf \left\{\lambda \in(0, \infty): \int_{\Omega} \Phi\left(\frac{|f(x)|}{\lambda}\right) \omega(x) d x \leq 1\right\} .
$$

If $p \in(0,1]$ and $\Phi(t):=t^{p}$ for all $t \in(0, \infty)$, we then denote $L_{\omega}^{\Phi}(\Omega)$ simply by $L_{\omega}^{p}(\Omega)$.

Definition 3.1. Let $\Phi$ satisfy Assumption (A), $\omega \in A_{\infty}\left(\mathbb{R}^{n}\right), \Omega$ be either $\mathbb{R}^{n}$ or a strongly Lipschitz domain of $\mathbb{R}^{n}$ and $L$ be as in (2.7). Let $R_{0} \in(0, \infty)$. For all $f \in L^{2}(\Omega)$ and $x \in \Omega$, let

$$
\mathcal{N}_{h}^{\text {loc, } R_{0}}(f)(x):=\sup _{y \in \Omega, t \in\left(0, R_{0}\right],|y-x|<t}\left|e^{-t^{2} L}(f)(y)\right| .
$$


When $R_{0}:=1$, denote $\mathcal{N}_{h}^{\text {loc, } R_{0}}(f)$ simply by $\mathcal{N}_{h}^{\text {loc }}(f)$. A function $f \in L^{2}(\Omega)$ is said to be in $\widetilde{h}_{\mathcal{N}_{h}, \omega}^{\Phi}(\Omega)$ if $\mathcal{N}_{h}^{\text {loc }}(f) \in L_{\omega}^{\Phi}(\Omega)$; moreover, define

$$
\begin{aligned}
\|f\|_{h_{\mathcal{N}_{h}, \omega}^{\text {T. }}(\Omega)} & :=\left\|\mathcal{N}_{h}^{\text {loc }}(f)\right\|_{L_{\omega}^{\Phi}(\Omega)} \\
& :=\inf \left\{\lambda \in(0, \infty): \int_{\Omega} \Phi\left(\frac{\mathcal{N}_{h}^{\text {loc }}(f)(x)}{\lambda}\right) \omega(x) d x \leq 1\right\} .
\end{aligned}
$$

The weighted local Orlicz-Hardy space $h_{\mathcal{N}_{h}, \omega}^{\Phi}(\Omega)$ is defined to be the completion of $\widetilde{h}_{\mathcal{N}_{h}, \omega}^{\Phi}(\Omega)$ in the quasi-norm $\|\cdot\|_{h_{\mathcal{N}_{h}, \omega}^{\Phi}(\Omega)}$.

Remark 3.2. (i) It is easy to see that $\|\cdot\|_{h_{\mathcal{N}_{h}, \omega}^{\text {T }}(\Omega)}$ is a quasi-norm.

(ii) From the Aoki-Rolewicz theorem in [5, 72, it follows that there exist a quasi-norm $\|\cdot\|$ on $\widetilde{h}_{\mathcal{N}_{h}, \omega}(\Omega)$ and $\gamma \in(0,1]$ such that for all $f \in \widetilde{h}_{\mathcal{N}_{h}, \omega}^{\Phi}(\Omega)$,

$$
\|f \mid\| \sim\|f\|_{h_{\mathcal{N}_{h}, \omega}^{\Phi}(\Omega)},
$$

and that for any sequence $\left\{f_{j}\right\}_{j} \subset \widetilde{h}_{\mathcal{N}_{h}, \omega}(\Omega)$,

$$
\left\|\sum_{j} f_{j}\right\|\left\|^{\gamma} \leq \sum_{j} \mid\right\| f_{j} \|^{\gamma} .
$$

By the theorem of completion of Yosida [89, p. 56], it follows that $\left(\widetilde{h}_{\mathcal{N}_{h}, \omega}^{\Phi}(\Omega), \mid\|\cdot\|\right)$ has a completion space $\left(h_{\mathcal{N}_{h}, \omega}^{\Phi}(\Omega),|\|\cdot \mid\|)\right.$; namely, for any $f \in\left(h_{\mathcal{N}_{h}, \omega}^{\Phi}(\Omega),|\|\cdot \mid\|)\right.$, there exists a Cauchy sequence $\left\{f_{k}\right\}_{k=1}^{\infty} \subset\left(\widetilde{h}_{\mathcal{N}_{h}, \omega}^{\Phi}(\Omega),\|\| \cdot \|\right)$ such that

$$
\lim _{k \rightarrow \infty}\left|\left\|f_{k}-f \mid\right\|=0\right. \text {. }
$$

Moreover, if $\left\{f_{k}\right\}_{k=1}^{\infty}$ is a Cauchy sequence in $\left(\widetilde{h}_{\mathcal{N}_{h}, \omega}^{\Phi}(\Omega),|\|\cdot \mid\|)\right.$, then there exists a unique $f \in\left(h_{\mathcal{N}_{h}, \omega}^{\Phi}(\Omega),|\|\cdot \mid\|)\right.$ such that

$$
\lim _{k \rightarrow \infty}\left|\left\|f_{k}-f \mid\right\|=0 .\right.
$$

Furthermore, by $\mid\|f\| \sim\|f\|_{h_{\mathcal{N}_{h}, \omega}^{\Phi}(\Omega)}$ for all $f \in \widetilde{h}_{\mathcal{N}_{h}, \omega}^{\Phi}(\Omega)$, we know that the spaces $\left(h_{\mathcal{N}_{h}, \omega}^{\Phi}(\Omega),\|\cdot\|_{h_{\mathcal{N}_{h}, \omega}^{\Phi}(\Omega)}\right)$ and $\left(h_{\mathcal{N}_{h}, \omega}^{\Phi}(\Omega),|\|\cdot \mid\|)\right.$ coincide with equivalent quasi-norms.

In what follows, $Q(x, t)$ denotes the closed cube of $\mathbb{R}^{n}$ centered at $x$ and of sidelength $t$ with sides parallel to the axes. Similarly, given $Q:=Q(x, t)$ and $\lambda \in$ $(0, \infty)$, we write $\lambda Q$ for the $\lambda$-dilated cube, which is the cube with the same center $x$ and with sidelength $\lambda t$. Let $R_{0} \in(0, \infty)$. For any $f \in L^{2}(\Omega)$ and $x \in \Omega$, the Lusin area functions $S_{h, R_{0}}^{\text {loc }}(f)(x)$ and $\widetilde{S}_{h, R_{0}}^{\text {loc }}(f)(x)$ associated with $\left\{e^{-t^{2} L}\right\}_{t \geq 0}$ are respectively defined by

$$
S_{h, R_{0}}^{\mathrm{loc}}(f)(x):=\left[\int_{\Gamma^{R_{0}(x)}}\left|t^{2} L e^{-t^{2} L}(f)(y)\right|^{2} \frac{d y d t}{t|Q(x, t) \cap \Omega|}\right]^{\frac{1}{2}}
$$

and

$$
\widetilde{S}_{h, R_{0}}^{\mathrm{loc}}(f)(x):=\left[\int_{\Gamma^{R_{0}(x)}}\left|t \nabla e^{-t^{2} L}(f)(y)\right|^{2} \frac{d y d t}{t|Q(x, t) \cap \Omega|}\right]^{\frac{1}{2}}
$$


where $\Gamma^{R_{0}}(x)$ is the cone defined by

$$
\Gamma^{R_{0}}(x):=\left\{(y, t) \in \Omega \times\left(0, R_{0}\right]:|y-x|<t\right\} .
$$

When $R_{0}:=1$, we denote $S_{h, R_{0}}^{\text {loc }}$ and $\widetilde{S}_{h, R_{0}}^{\text {loc }}$ simply, respectively, by $S_{h}^{\text {loc }}$ and $\widetilde{S}_{h}^{\text {loc }}$.

To give the definitions of the weighted local Orlicz-Hardy spaces associated with the Lusin area functions $S_{h}^{\text {loc }}$ and $\widetilde{S}_{h}^{\text {loc }}$, we need the following notation.

In what follows, denote by $\mathcal{Q}$ the set of all unit cubes of $\mathbb{R}^{n}$ whose interiors are disjoint. Let $\Omega$ be either $\mathbb{R}^{n}$ or a strongly Lipschitz domain of $\mathbb{R}^{n}$. For any given $Q \in \mathcal{Q}$ satisfying $Q \cap \Omega \neq \emptyset$, if the center of $Q, x_{Q} \in Q \cap \Omega$, let $\widetilde{Q}:=Q$; if the center of $Q, x_{Q} \in Q \cap \Omega^{\complement}$, take the cube $\widetilde{Q} \subset \mathbb{R}^{n}$ satisfying $l(\widetilde{Q})=2, Q \subset \widetilde{Q}$ and the center of $\widetilde{Q}, x_{\widetilde{Q}} \in \widetilde{Q} \cap \Omega$, where and in what follows, $\Omega^{\complement}$ denotes the complement of $\Omega$ in $\mathbb{R}^{n}$. Denote by $\mathcal{Q}_{\Omega}$ the set of all cubes $\widetilde{Q}$ as above. For any given measurable subset $E$ of $\mathbb{R}^{n}$ satisfying $|E|<\infty$ and $f \in L_{\text {loc }}^{1}\left(\mathbb{R}^{n}\right)$, let

$$
m_{E}(f):=\frac{1}{|E|} \int_{E} f(y) d y
$$

where and in what follows, the space $L_{\text {loc }}^{1}\left(\mathbb{R}^{n}\right)$ denotes the set of all locally integrable functions on $\mathbb{R}^{n}$.

Definition 3.3. Let $\Phi$ satisfy Assumption (A), $\omega \in A_{\infty}\left(\mathbb{R}^{n}\right), \Omega$ be either $\mathbb{R}^{n}$ or a strongly Lipschitz domain of $\mathbb{R}^{n}$ and $L$ be as in (2.7). A function $f \in L^{2}(\Omega)$ is said to be in $\widetilde{h}_{\omega, S_{h}}^{\Phi}(\Omega)$ if $S_{h}^{\text {loc }}(f) \in L_{\omega}^{\Phi}(\Omega)$ and

$$
\sum_{Q \in \mathcal{Q}_{\Omega}} \omega(Q \cap \Omega) \Phi\left(m_{Q \cap \Omega}\left(\left|e^{-L}(f)\right|\right)\right)<\infty .
$$

Furthermore, define

$$
\begin{aligned}
\|f\|_{h_{\omega, S_{h}}^{\Phi}(\Omega)}: & \inf \left\{\lambda \in(0, \infty): \int_{\Omega} \Phi\left(\frac{S_{h}^{\operatorname{loc}}(f)(x)}{\lambda}\right) \omega(x) d x \leq 1\right\} \\
& +\inf \left\{\lambda \in(0, \infty): \sum_{Q \in \mathcal{Q}_{\Omega}} \omega(Q \cap \Omega) \Phi\left(\frac{m_{Q \cap \Omega}\left(\left|e^{-L}(f)\right|\right)}{\lambda}\right) \leq 1\right\} .
\end{aligned}
$$

The weighted local Orlicz-Hardy space $h_{S_{h}, \omega}^{\Phi}(\Omega)$ is defined to be the completion of $\widetilde{h}_{S_{h}, \omega}^{\Phi}(\Omega)$ in the norm $\|\cdot\|_{h_{S_{h}, \omega}^{\Phi}(\Omega)}$.

The weighted local Orlicz-Hardy space $h_{\widetilde{S}_{h}, \omega}^{\Phi}(\Omega)$ is defined via replacing $S_{h}^{\text {loc in }}$ the definition of $h_{S_{h}, \omega}^{\Phi}(\Omega)$ by $\widetilde{S}_{h}^{\text {loc }}$.

In this section, we present the proof of Theorem 1.4. To this end, we need some auxiliary area functions as follows. Let $R_{0} \in(0, \infty), \alpha \in(0, \infty), \epsilon, R \in\left(0, R_{0}\right)$ and $\epsilon<R$. For all given $f \in L^{2}(\Omega)$ and $x \in \Omega$, let

$$
\widetilde{S}_{h, R_{0}}^{\text {loc, } \alpha}(f)(x):=\left[\int_{\Gamma_{\alpha}^{R_{0}(x)}}\left|t \nabla e^{-t^{2} L}(f)(y)\right|^{2} \frac{d y d t}{t|Q(x, t) \cap \Omega|}\right]^{\frac{1}{2}}
$$

and

$$
\widetilde{S}_{h, R_{0}}^{\epsilon, R, \alpha}(f)(x):=\left[\int_{\Gamma_{\alpha}^{\epsilon, R}(x)}\left|t \nabla e^{-t^{2} L}(f)(y)\right|^{2} \frac{d y d t}{t|Q(x, t) \cap \Omega|}\right]^{\frac{1}{2}}
$$


where and in what follows, for all $x \in \Omega, \Gamma_{\alpha}^{R_{0}}(x)$ and $\Gamma_{\alpha}^{\epsilon, R}(x)$ are the local cone and the truncated cone defined, respectively, by

$$
\Gamma_{\alpha}^{R_{0}}(x):=\left\{(y, t) \in \Omega \times\left(0, R_{0}\right]:|y-x|<\alpha t\right\}
$$

and

$$
\Gamma_{\alpha}^{\epsilon, R}(x):=\{(y, t) \in \Omega \times(\epsilon, R]:|y-x|<\alpha t\}
$$

for $\alpha \in(0, \infty)$ and $0<\epsilon<R<R_{0}$. When $\alpha=1$, we denote $\widetilde{S}_{h, R_{0}}^{\text {loc, }}(f), \widetilde{S}_{h, R_{0}}^{\epsilon, R}(f)$ and $\Gamma_{\alpha}^{R_{0}}(x)$ simply, respectively, by $\widetilde{S}_{h, R_{0}}^{\text {loc }}(f), \widetilde{S}_{h, R_{0}}^{\epsilon, R}(f)$ and $\Gamma^{R_{0}}(x)$. To show Theorem 1.4, we first establish the following Proposition 3.4.

Proposition 3.4. Let $\Phi$ satisfy Assumption $(A), \omega \in A_{\infty}\left(\mathbb{R}^{n}\right), R_{0} \in(0, \infty)$ and $L$ be as in (2.7). Let $q_{\omega}, \mu$ and $p_{\Phi}^{-}$be respectively as in (2.5), (2.9) and (2.12). Let $\Omega$ be either $\mathbb{R}^{n}$ or a strongly Lipschitz domain of $\mathbb{R}^{n}$. Assume that $q_{\omega}, \mu$ and $p_{\Phi}^{-}$ satisfy the inequality $q_{\omega} / p_{\Phi}^{-}<(n+\mu) / n$, and that the semigroup generated by $L$ has the Gaussian property $\left(G_{1}\right)$.

(i) If $\Omega:=\mathbb{R}^{n}$, then there exists a positive constant $C$ such that for all $f \in$ $h_{\omega}^{\Phi}\left(\mathbb{R}^{n}\right) \cap L^{2}\left(\mathbb{R}^{n}\right)$,

$$
\left\|\mathcal{N}_{h}^{\text {loc, } R_{0}}(f)\right\|_{L_{\omega}^{\Phi}\left(\mathbb{R}^{n}\right)} \leq C\|f\|_{h_{\omega}^{\Phi}\left(\mathbb{R}^{n}\right)} .
$$

(ii) Under DBC, there exists a positive constant $C$ such that for all $f \in h_{\omega, r}^{\Phi}(\Omega) \cap$ $L^{2}(\Omega)$,

$$
\left\|\mathcal{N}_{h}^{\mathrm{loc}, R_{0}}(f)\right\|_{L_{\omega}^{\Phi}(\Omega)} \leq C\|f\|_{h_{\omega, r}^{\Phi}(\Omega)} .
$$

(iii) Under NBC, there exists a positive constant $C$ such that for all $f \in h_{\omega, z}^{\Phi}(\Omega) \cap$ $L^{2}(\Omega)$,

$$
\left\|\mathcal{N}_{h}^{\mathrm{loc}, R_{0}}(f)\right\|_{L_{\omega}^{\Phi}(\Omega)} \leq C\|f\|_{h_{\omega, z}^{\Phi}(\Omega)} .
$$

To show Proposition 3.4, we need the atomic decomposition characterization of the weighted local Orlicz-Hardy space $h_{\omega}^{\Phi}\left(\mathbb{R}^{n}\right)$ established in [86]. To state this, we begin with the notions of $(\rho, q, s)_{\omega}$-atoms and the weighted atomic local OrliczHardy space $h_{\omega}^{\rho, q, s}\left(\mathbb{R}^{n}\right)$.

Definition 3.5. Let $\Phi$ satisfy Assumption (A), $\omega \in A_{\infty}\left(\mathbb{R}^{n}\right), p_{\Phi}^{-}, q_{\omega}$ and $\rho$ be respectively as in (2.12), (2.5) and (2.14). A triplet $(\rho, q, s)_{\omega}$ is called admissible if $q \in\left(q_{\omega}, \infty\right]$ and $s \in \mathbb{Z}_{+}$with

$$
s \geq\left\lfloor n\left(\frac{q_{\omega}}{p_{\Phi}^{-}}-1\right)\right\rfloor .
$$

A function $a$ on $\mathbb{R}^{n}$ is called a $(\rho, q, s)_{\omega}$-atom if there exists a cube $Q \subset \mathbb{R}^{n}$ such that

(i) $\operatorname{supp}(a) \subset Q$;

(ii)

$$
\|a\|_{L_{\omega}^{q}\left(\mathbb{R}^{n}\right)} \leq[\omega(Q)]^{\frac{1}{q}-1}[\rho(\omega(Q))]^{-1} ;
$$

(iii) for all $\alpha \in \mathbb{Z}_{+}^{n}$ with $|\alpha| \leq s$, when $l(Q)<1$,

$$
\int_{\mathbb{R}^{n}} a(x) x^{\alpha} d x=0 .
$$


Definition 3.6. Let $\Phi$ satisfy Assumption (A), $\omega \in A_{\infty}\left(\mathbb{R}^{n}\right), q_{\omega}, \rho$ be respectively as in (2.5) and (2.14), and $(\rho, q, s)_{\omega}$ admissible. The weighted atomic local OrliczHardy space $h_{\omega}^{\rho, q, s}\left(\mathbb{R}^{n}\right)$ is defined to be the set of all $f \in \mathcal{D}^{\prime}\left(\mathbb{R}^{n}\right)$ satisfying that

$$
f=\sum_{i=1}^{\infty} \lambda_{i} a_{i}
$$

in $\mathcal{D}^{\prime}\left(\mathbb{R}^{n}\right)$, where $\left\{a_{i}\right\}_{i \in \mathbb{N}}$ are $(\rho, q, s)_{\omega}$-atoms with $\operatorname{supp}\left(a_{i}\right) \subset Q_{i},\left\{\lambda_{i}\right\}_{i \in \mathbb{N}} \subset \mathbb{C}$, and

$$
\sum_{i=1}^{\infty} \omega\left(Q_{i}\right) \Phi\left(\frac{\left|\lambda_{i}\right|}{\omega\left(Q_{i}\right) \rho\left(\omega\left(Q_{i}\right)\right)}\right)<\infty
$$

Moreover, letting

$$
\Lambda\left(\left\{\lambda_{i} a_{i}\right\}_{i \in \mathbb{N}}\right):=\inf \left\{\lambda \in(0, \infty): \sum_{i=1}^{\infty} \omega\left(Q_{i}\right) \Phi\left(\frac{\left|\lambda_{i}\right|}{\lambda \omega\left(Q_{i}\right) \rho\left(\omega\left(Q_{i}\right)\right)}\right) \leq 1\right\},
$$

the quasi-norm of $f \in h_{\omega}^{\rho, q, s}\left(\mathbb{R}^{n}\right)$ is defined by

$$
\|f\|_{h_{\omega}^{\rho, q, s}\left(\mathbb{R}^{n}\right)}:=\inf \left\{\Lambda\left(\left\{\lambda_{i} a_{i}\right\}_{i \in \mathbb{N}}\right)\right\},
$$

where the infimum is taken over all the decompositions of $f$ as above.

The $(\rho, q, s)_{\omega}$-atom and the weighted atomic local Orlicz-Hardy space $h_{\omega}^{\rho, q, s}\left(\mathbb{R}^{n}\right)$ were introduced in [86, in which the following Lemma 3.7 was also obtained (see [86, Theorem 5.6]).

Lemma 3.7. Let $\Phi$ satisfy Assumption (A), $\omega \in A_{\infty}\left(\mathbb{R}^{n}\right), q_{\omega}, p_{\Phi}^{-}$and $\rho$ be respectively as in (2.5), (2.12) and (2.14). If $q \in\left(q_{\omega}, \infty\right]$ and integer $s$ satisfies $s \geq\left\lfloor n\left(\frac{q_{\omega}}{p_{\Phi}^{-}}-1\right)\right\rfloor$, then

$$
h_{\omega}^{\rho, q, s}\left(\mathbb{R}^{n}\right)=h_{\omega}^{\Phi}\left(\mathbb{R}^{n}\right)
$$

with equivalent quasi-norms.

We point out that in [86. Theorem 5.6], the assumption $p_{\Phi}^{-} \in(0,1]$ was replaced by the assumption $p_{\Phi} \in(0,1]$, which, however, can be relaxed; see Remark 2.7

Now we prove Proposition 3.4 by applying Lemma 3.7

Proof of Proposition 3.4. We first prove Proposition 3.4(i). To this end, it suffices to prove that for any $(\rho, q, 0)$-atom $a$ supported in $Q_{0}:=Q\left(x_{0}, r_{0}\right)$ with some $q \in\left(q_{\omega}, \infty\right]$ and any $\lambda \in \mathbb{C}$,

$$
\int_{\mathbb{R}^{n}} \Phi\left(\mathcal{N}_{h}^{\text {loc, } R_{0}}(\lambda a)(x)\right) \omega(x) d x \lesssim \omega\left(Q_{0}\right) \Phi\left(\frac{|\lambda|}{\omega\left(Q_{0}\right) \rho\left(\omega\left(Q_{0}\right)\right)}\right) .
$$

Indeed, if (3.2) holds, by Lemma 3.7 and an argument in [86, p. 43], we know that for any $f \in h_{\omega}^{\Phi}\left(\mathbb{R}^{n}\right) \cap L^{2}\left(\mathbb{R}^{n}\right)$, there exist $\left\{\lambda_{i}\right\}_{i} \subset \mathbb{C}$ and a sequence $\left\{a_{i}\right\}_{i}$ of $(\rho, q, 0)$-atoms such that

$$
f=\sum_{i} \lambda_{i} a_{i} \text { in } L^{2}\left(\mathbb{R}^{n}\right)
$$


and

$$
\begin{aligned}
\Lambda\left(\left\{\lambda_{i} a_{i}\right\}_{i}\right) & :=\inf \left\{\lambda \in(0, \infty): \sum_{i} \omega\left(Q_{i}\right) \Phi\left(\frac{\left|\lambda_{i}\right|}{\lambda \omega\left(Q_{i}\right) \rho\left(\omega\left(Q_{i}\right)\right)}\right) \leq 1\right\} \\
& \lesssim\|f\|_{h_{\omega}^{\Phi}\left(\mathbb{R}^{n}\right)}
\end{aligned}
$$

where for each $i, \operatorname{supp}\left(a_{i}\right) \subset Q_{i}$. By (3.3), we conclude that for all $x \in \mathbb{R}^{n}$,

$$
\mathcal{N}_{h}^{\text {loc, } R_{0}}(f)(x) \leq \sum_{i} \mathcal{N}_{h}^{\text {loc, } R_{0}}\left(\lambda_{i} a_{i}\right)(x),
$$

which, together with the assumptions that $\Phi$ is strictly increasing, subadditive and continuous, and that for any $\lambda \in(0, \infty)$ and each $i$,

$$
\mathcal{N}_{h}^{\text {loc, } R_{0}}(f / \lambda)=\mathcal{N}_{h}^{\text {loc }, R_{0}}(f) / \lambda, \quad \mathcal{N}_{h}^{\text {loc }, R_{0}}\left(a_{i} / \lambda\right)=\mathcal{N}_{h}^{\text {loc }, R_{0}}\left(a_{i}\right) / \lambda
$$

and (3.2), implies that for any $\lambda \in(0, \infty)$,

$$
\begin{aligned}
& \int_{\mathbb{R}^{n}} \Phi\left(\frac{\mathcal{N}_{h}^{\text {loc }, R_{0}}(f)(x)}{\lambda}\right) \omega(x) d x \\
& \quad=\int_{\mathbb{R}^{n}} \Phi\left(\mathcal{N}_{h}^{\text {loc }, R_{0}}\left(\frac{f}{\lambda}\right)(x)\right) \omega(x) d x \\
& \quad \leq \sum_{i} \int_{\mathbb{R}^{n}} \Phi\left(\mathcal{N}_{h}^{\text {loc }, R_{0}}\left(\frac{\lambda_{i} a_{i}}{\lambda}\right)(x)\right) \omega(x) d x \\
& \quad \lesssim \sum_{i} \omega\left(Q_{i}\right) \Phi\left(\frac{\left|\lambda_{i}\right|}{\lambda \omega\left(Q_{i}\right) \rho\left(\omega\left(Q_{i}\right)\right)}\right) .
\end{aligned}
$$

From this and (3.4), we deduce that

which is as desired.

$$
\left\|\mathcal{N}_{h}^{\text {loc, } R_{0}}(f)\right\|_{L_{\omega}^{\Phi}\left(\mathbb{R}^{n}\right)} \lesssim\|f\|_{h_{\omega}^{\Phi}\left(\mathbb{R}^{n}\right)},
$$

Now we prove (3.2). Let $\widetilde{Q}_{0}:=4\left(R_{0}+1\right) Q_{0}$. Then we write

$$
\begin{aligned}
\int_{\mathbb{R}^{n}} \Phi\left(\mathcal{N}_{h}^{\text {loc }, R_{0}}(\lambda a)(x)\right) \omega(x) d x \\
\quad=\int_{\widetilde{Q}_{0}} \Phi\left(\mathcal{N}_{h}^{\text {loc }, R_{0}}(\lambda a)(x)\right) \omega(x) d x+\int_{\widetilde{Q}_{0}^{\mathrm{c}}} \cdots=: \mathrm{I}_{1}+\mathrm{I}_{2} .
\end{aligned}
$$

We first estimate $\mathrm{I}_{1}$. From the assumption that $\frac{q_{\omega}}{p_{\Phi}^{-}}<\frac{n+\mu}{n}$, we deduce that there exist $q \in\left(q_{\omega}, \infty\right), p_{0} \in\left(0, p_{\Phi}^{-}\right)$and $\widetilde{\mu} \in(0, \mu)$ such that $\Phi$ is of lower type $p_{0}$ and

$$
\frac{q}{p_{0}}<\frac{n+\widetilde{\mu}}{n}
$$

Since $q \in\left(q_{\omega}, \infty\right)$, by the definition of $q_{\omega}$, we know that $\omega \in A_{q}\left(\mathbb{R}^{n}\right)$. Since $\Phi$ is concave, by Jensen's inequality, Hölder's inequality, (vii) and (iii) of Lemma 2.2. we have

$$
\begin{aligned}
\mathrm{I}_{1} & \leq \omega\left(\widetilde{Q}_{0}\right) \Phi\left(\frac{1}{\omega\left(\widetilde{Q}_{0}\right)} \int_{\widetilde{Q}_{0}} \mathcal{N}_{h}^{\text {loc, } R_{0}}(\lambda a)(x) \omega(x) d x\right) \\
& \leq \omega\left(\widetilde{Q}_{0}\right) \Phi\left(\frac{1}{\left[\omega\left(\widetilde{Q}_{0}\right)\right]^{\frac{1}{q}}}\left\{\int_{\widetilde{Q}_{0}}\left[\mathcal{N}_{h}^{\text {loc, } R_{0}}(\lambda a)(x)\right]^{q} \omega(x) d x\right\}^{\frac{1}{q}}\right)
\end{aligned}
$$




$$
\begin{aligned}
& \lesssim \omega\left(\widetilde{Q}_{0}\right) \Phi\left(\frac{1}{\left[\omega\left(\widetilde{Q}_{0}\right)\right]^{\frac{1}{q}}}|\lambda|\|a\|_{L_{\omega}^{q}\left(\mathbb{R}^{n}\right)}\right) \\
& \lesssim \omega\left(\widetilde{Q}_{0}\right) \Phi\left(\frac{|\lambda|}{\omega\left(Q_{0}\right) \rho\left(\omega\left(Q_{0}\right)\right)}\right) \lesssim \omega\left(Q_{0}\right) \Phi\left(\frac{|\lambda|}{\omega\left(Q_{0}\right) \rho\left(\omega\left(Q_{0}\right)\right)}\right)
\end{aligned}
$$

which is as desired.

Now we deal with $\mathrm{I}_{2}$ by considering the following two cases for $Q_{0}$.

Case 1) $l\left(Q_{0}\right)<1$. In this case, let $x \in\left(\widetilde{Q}_{0}\right)^{\complement}, t \in\left(0, R_{0}\right]$ and $y \in \mathbb{R}^{n}$ satisfy $|x-y|<t$. Let $\widetilde{\mu}$ be as in (3.6). By an argument similar to that in [87, p. 18], we know that for all $z \in Q_{0}$,

$$
\left|K_{t^{2}}(y, z)-K_{t^{2}}\left(y, x_{0}\right)\right| \lesssim \frac{\left|z-x_{0}\right|^{\widetilde{\mu}}}{\left|x-x_{0}\right|^{n+\widetilde{\mu}}},
$$

which, together with the moment condition of $a$, Hölder's inequality and (2.1), implies that

$$
\begin{aligned}
\left|e^{-t^{2} L}(\lambda a)(y)\right| & =\left|\int_{\mathbb{R}^{n}} \lambda\left[K_{t^{2}}(y, z)-K_{t^{2}}\left(y, x_{0}\right)\right] a(z) d z\right| \\
& \lesssim \int_{\mathbb{R}^{n}} \frac{|\lambda|\left|z-x_{0}\right|^{\widetilde{\mu}}}{\left|x-x_{0}\right|^{n+\widetilde{\mu}}}|a(z)| d z \\
& \lesssim|\lambda|\left|Q_{0}\right|^{\widetilde{\mu} / n}|| a \|_{L_{\omega}^{q}\left(\mathbb{R}^{n}\right)}\left\{\int_{Q_{0}}[\omega(x)]^{-q^{\prime} / q} d x\right\}^{\frac{1}{q^{\prime}}}\left|x-x_{0}\right|^{-(n+\widetilde{\mu})} \\
& \lesssim|\lambda|\left|Q_{0}\right|^{\frac{\tilde{\mu}}{n}}\|a\|_{L_{\omega}^{q}\left(\mathbb{R}^{n}\right)} \frac{\left|Q_{0}\right|}{\left[\omega\left(Q_{0}\right)\right]^{\frac{1}{q}}}\left|x-x_{0}\right|^{-(n+\widetilde{\mu})} \\
& \lesssim \frac{\left|\lambda \| Q_{0}\right|^{\frac{n+\widetilde{\mu}}{n}}}{\omega\left(Q_{0}\right) \rho\left(\omega\left(Q_{0}\right)\right)}\left|x-x_{0}\right|^{-(n+\widetilde{\mu})}
\end{aligned}
$$

where and in what follows, $\frac{1}{q}+\frac{1}{q^{\prime}}=1$. From this, it further follows that for all $x \in\left(\widetilde{Q}_{0}\right)^{\complement}$,

$$
\mathcal{N}_{h}^{\text {loc, } R_{0}}(\lambda a)(x) \lesssim \frac{|\lambda|\left|Q_{0}\right|^{\frac{n+\widetilde{\mu}}{n}}}{\omega\left(Q_{0}\right) \rho\left(\omega\left(Q_{0}\right)\right)}\left|x-x_{0}\right|^{-(n+\widetilde{\mu})},
$$

which, together with the lower type $p_{0}$ and upper type 1 properties of $\Phi$, Lemma 2.2 (iii) and (3.6), implies that

$$
\begin{aligned}
\mathrm{I}_{2} & \lesssim \int_{\left(\widetilde{Q}_{0}\right)^{\mathrm{C}}} \Phi\left(\frac{|\lambda|\left|Q_{0}\right|^{(n+\widetilde{\mu}) / n}}{\omega\left(Q_{0}\right) \rho\left(\omega\left(Q_{0}\right)\right)}\left|x-x_{0}\right|^{-(n+\widetilde{\mu})}\right) \omega(x) d x \\
& \lesssim \sum_{k=2}^{\infty} \int_{2^{k+1} Q_{0} \backslash 2^{k} Q_{0}} \Phi\left(2^{-k(n+\widetilde{\mu})}|\lambda|\left[\omega\left(Q_{0}\right) \rho\left(\omega\left(Q_{0}\right)\right)\right]^{-1}\right) \omega(x) d x \\
& \lesssim \sum_{k=2}^{\infty} 2^{-k\left[(n+\widetilde{\mu}) p_{0}-n q\right]} \omega\left(Q_{0}\right) \Phi\left(\frac{|\lambda|}{\omega\left(Q_{0}\right) \rho\left(\omega\left(Q_{0}\right)\right)}\right) \\
& \lesssim \omega\left(Q_{0}\right) \Phi\left(\frac{|\lambda|}{\omega\left(Q_{0}\right) \rho\left(\omega\left(Q_{0}\right)\right)}\right)
\end{aligned}
$$


Case 2) $l\left(Q_{0}\right) \geq 1$. In this case, let $x \in\left(\widetilde{Q}_{0}\right)^{\complement}, t \in\left(0, R_{0}\right]$ and $y \in \mathbb{R}^{n}$ satisfy $|x-y|<t$. Then for all $z \in Q_{0}$,

$$
\begin{aligned}
|y-z| & \geq|x-z|-|x-y| \geq\left|x-x_{0}\right|-\left|x_{0}-z\right|-|x-y| \\
& \geq\left|x-x_{0}\right|-\frac{1}{4\left(R_{0}+1\right)}\left|x-x_{0}\right|-\frac{R_{0}}{2\left(R_{0}+1\right)}\left|x-x_{0}\right|>\frac{1}{2}\left|x-x_{0}\right|,
\end{aligned}
$$

which, together with (2.8), Hölder's inequality and (2.1), implies that

$$
\begin{aligned}
\left|e^{-t^{2} L}(\lambda a)(y)\right| & =\left|\int_{Q_{0}} \lambda K_{t^{2}}(y, z) a(z) d z\right| \lesssim \int_{Q_{0}} \frac{|\lambda| t}{|y-z|^{n+1}}|a(z)| d z \\
& \lesssim \frac{|\lambda|}{\left|x-x_{0}\right|^{n+1}} \int_{Q_{0}}|a(z)| d z \lesssim|\lambda||| a \|_{L_{\omega}^{q}\left(\mathbb{R}^{n}\right)} \frac{\left|Q_{0}\right|}{\left[\omega\left(Q_{0}\right)\right]^{\frac{1}{q}}} \frac{1}{\left|x-x_{0}\right|^{n+1}} \\
& \lesssim|\lambda|\left|Q_{0}\right|\left[\omega\left(Q_{0}\right) \rho\left(\omega\left(Q_{0}\right)\right)\right]^{-1}\left|x-x_{0}\right|^{-(n+1)},
\end{aligned}
$$

where we omitted some computations similar to (3.8). From this, we infer that for all $x \in\left(\widetilde{Q}_{0}\right)^{\complement}$,

$$
\mathcal{N}_{h}^{\text {loc, } R_{0}}(\lambda a)(x) \lesssim|\lambda|\left|Q_{0}\right|\left[\omega\left(Q_{0}\right) \rho\left(\omega\left(Q_{0}\right)\right)\right]^{-1}\left|x-x_{0}\right|^{-(n+1)},
$$

which, together with the lower type $p_{0}$ and upper type 1 properties of $\Phi$, Lemma 2.2 (iii), $l\left(Q_{0}\right) \geq 1$ and (3.6), implies that

$$
\begin{aligned}
\mathrm{I}_{2} & \lesssim \int_{\left(\widetilde{Q}_{0}\right)^{\mathrm{C}}} \Phi\left(|\lambda|\left|Q_{0}\right|\left[\omega\left(Q_{0}\right) \rho\left(\omega\left(Q_{0}\right)\right)\right]^{-1}\left|x-x_{0}\right|^{-(n+1)}\right) \omega(x) d x \\
& \lesssim \sum_{k=2}^{\infty} \int_{2^{k+1} Q_{0} \backslash 2^{k} Q_{0}} \Phi\left(2^{-k(n+1)}|\lambda|\left[\omega\left(Q_{0}\right) \rho\left(\omega\left(Q_{0}\right)\right)\right]^{-1}\right) \omega(x) d x \\
& \lesssim \sum_{k=2}^{\infty} 2^{-k\left[(n+1) p_{0}-n q\right]} \omega\left(Q_{0}\right) \Phi\left(\frac{|\lambda|}{\omega\left(Q_{0}\right) \rho\left(\omega\left(Q_{0}\right)\right)}\right) \\
& \lesssim \omega\left(Q_{0}\right) \Phi\left(\frac{|\lambda|}{\omega\left(Q_{0}\right) \rho\left(\omega\left(Q_{0}\right)\right)}\right) .
\end{aligned}
$$

Thus, by (3.5), (3.7), (3.9) and (3.10), we conclude that

$$
\int_{\mathbb{R}^{n}} \Phi\left(\mathcal{N}_{h}^{\text {loc, } R_{0}}(\lambda a)(x)\right) \omega(x) d x \lesssim \omega\left(Q_{0}\right) \Phi\left(\frac{|\lambda|}{\omega\left(Q_{0}\right) \rho\left(\omega\left(Q_{0}\right)\right)}\right),
$$

which implies that (3.2) holds and hence completes the proof of Proposition 3.4(i).

Now we prove Proposition 3.4(ii). Let $f \in h_{\omega, r}^{\Phi}(\Omega) \cap L^{2}(\Omega)$. By the definition of $h_{\omega, r}^{\Phi}(\Omega)$, we know that there exists $\widetilde{f} \in h_{\omega}^{\Phi}\left(\mathbb{R}^{n}\right)$ such that $\left.\widetilde{f}\right|_{\Omega}=f$ and

$$
\|\widetilde{f}\|_{h_{\omega}^{\Phi}\left(\mathbb{R}^{n}\right)} \lesssim\|f\|_{h_{\omega, r}^{\Phi}(\Omega)} .
$$

Let $q$ be as in (3.6). To show Proposition 3.4(ii), we only need to prove that for any $(\rho, q, 0)_{\omega}$-atom $a$ supported in $Q_{0}:=Q\left(x_{0}, r_{0}\right)$ and $\lambda \in \mathbb{C}$,

$$
\int_{\Omega} \Phi\left(\mathcal{N}_{h}^{\text {loc, } R_{0}}(\lambda a)(x)\right) \omega(x) d x \lesssim \omega\left(Q_{0}\right) \Phi\left(\frac{|\lambda|}{\omega\left(Q_{0}\right) \rho\left(\omega\left(Q_{0}\right)\right)}\right) .
$$

Indeed, since $\tilde{f} \in h_{\omega}^{\Phi}\left(\mathbb{R}^{n}\right)$, by Lemma 3.7, there exist $\left\{\lambda_{i}\right\}_{i} \subset \mathbb{C}$ and a sequence $\left\{a_{i}\right\}_{i}$ of $(\rho, q, 0)$-atoms such that $\tilde{f}=\sum_{i} \lambda_{i} a_{i}$ in $\mathcal{D}^{\prime}\left(\mathbb{R}^{n}\right)$ and

$$
\Lambda\left(\left\{\lambda_{i} a_{i}\right\}_{i}\right) \sim\|\widetilde{f}\|_{h_{\omega}^{\Phi}\left(\mathbb{R}^{n}\right)} .
$$


Moreover, by the proof of [86, Theorem 5.1], we know that the supports of $\left\{a_{i}\right\}_{i}$ are of finite intersection property. By this, $f \in L^{2}(\Omega)$,

$$
\tilde{f}=\sum_{i} \lambda_{i} a_{i}
$$

in $\mathcal{D}^{\prime}\left(\mathbb{R}^{n}\right)$ and $\left.\widetilde{f}\right|_{\Omega}=f$, we see that $f=\sum_{i} \lambda_{i} a_{i}$ almost everywhere on $\Omega$, which further implies that

$$
\int_{\Omega} K_{t^{2}}(x, y) f(y) d y=\sum_{i} \lambda_{i} \int_{\Omega} K_{t^{2}}(x, y) a_{i}(y) d y .
$$

From this, we deduce that for all $x \in \Omega$,

$$
\mathcal{N}_{h}^{\text {loc, } R_{0}}(f)(x) \leq \sum_{i} \mathcal{N}_{h}^{\text {loc, } R_{0}}\left(\lambda_{i} a_{i}\right)(x),
$$

which, together with the assumptions that $\Phi$ is strictly increasing, subadditive and continuous, and that for any $\lambda \in(0, \infty)$ and each $i$,

$$
\mathcal{N}_{h}^{\text {loc, } R_{0}}(f / \lambda)=\mathcal{N}_{h}^{\text {loc }, R_{0}}(f) / \lambda, \quad \mathcal{N}_{h}^{\text {loc }, R_{0}}\left(a_{i} / \lambda\right)=\mathcal{N}_{h}^{\text {loc }, R_{0}}\left(a_{i}\right) / \lambda
$$

and (3.12), implies that for any $\lambda \in(0, \infty)$,

$$
\begin{aligned}
& \int_{\Omega} \Phi\left(\frac{\mathcal{N}_{h}^{\mathrm{loc}, R_{0}}(f)(x)}{\lambda}\right) \omega(x) d x \\
& \quad=\int_{\Omega} \Phi\left(\mathcal{N}_{h}^{\mathrm{loc}, R_{0}}\left(\frac{f}{\lambda}\right)(x)\right) \omega(x) d x \\
& \quad \leq \sum_{i} \int_{\Omega} \Phi\left(\mathcal{N}_{h}^{\mathrm{loc}, R_{0}}\left(\frac{\lambda_{i} a_{i}}{\lambda}\right)(x)\right) \omega(x) d x \\
& \quad \lesssim \sum_{i} \omega\left(Q_{i}\right) \Phi\left(\frac{\left|\lambda_{i}\right|}{\lambda \omega\left(Q_{i}\right) \rho\left(\omega\left(Q_{i}\right)\right)}\right),
\end{aligned}
$$

where for each $i, \operatorname{supp}\left(a_{i}\right) \subset Q_{i}$. From this, Lemma 3.7 and 3.11), we deduce that

$$
\left\|\mathcal{N}_{h}^{\text {loc, } R_{0}}(f)\right\|_{L_{\omega}^{\Phi}(\Omega)} \lesssim \Lambda\left(\left\{\lambda_{i} a_{i}\right\}_{i}\right) \sim\|\tilde{f}\|_{h_{\omega}^{\Phi}\left(\mathbb{R}^{n}\right)} \lesssim\|f\|_{h_{\omega, r}^{\Phi}(\Omega)},
$$

which, together with the arbitrariness of $f \in h_{\omega, r}^{\Phi}(\Omega) \cap L^{2}(\Omega)$, implies the conclusions of Proposition 3.4(ii).

It is easy to see that for all $x \in \Omega$,

$$
e^{-t^{2} L}(\lambda a)(x)=\int_{Q_{0} \cap \Omega} \lambda K_{t^{2}}(x, y) a(y) d y .
$$

Now we show (3.12) by considering the following three cases for $Q_{0}$.

Case i) $Q_{0} \cap \Omega=\emptyset$. In this case, by (3.13), we know that $\mathcal{N}_{h}^{\text {loc, } R_{0}}(\lambda a)(x)=0$ for all $x \in \Omega$. From this, it follows that (3.12) holds.

Case ii) $Q_{0} \subset \Omega$. In this case, the proof of (3.12) is similar to that of (3.2). We omit the details.

Case iii) $Q_{0} \cap \partial \Omega \neq \emptyset$. In this case, recall that for any $x \in \Omega, t \in(0, \infty)$ and $y \in \partial \Omega, K_{t}(x, y)=0$ (see, for example, [8, p. 156]). Take $y_{0} \in Q_{0} \cap \partial \Omega$. Then we 
see that for any $x \in \Omega$ and $t \in\left(0, R_{0}\right], K_{t^{2}}\left(x, y_{0}\right)=0$, which further implies that for any $x \in \Omega$,

$$
e^{-t^{2} L}(\lambda a)(x)=\int_{Q_{0} \cap \Omega} \lambda\left[K_{t^{2}}(x, y)-K_{t^{2}}\left(x, y_{0}\right)\right] a(y) d y
$$

provided $l\left(Q_{0}\right)<1$. The remaining estimates are similar to those of Proposition 3.4(i). We omit the details, which completes the proof of Proposition 3.4(ii).

Finally we give the proof of Proposition 3.4(iii). Let $f \in h_{\omega, z}^{\Phi}(\Omega) \cap L^{2}(\Omega)$ and $\widetilde{f}$ be the zero extension out of $\Omega$ of $f$. Then $\tilde{f} \in h_{\omega, z}^{\Phi}\left(\mathbb{R}^{n}\right) \cap L^{2}\left(\mathbb{R}^{n}\right)$. For all $t \in\left(0, R_{0}\right], x \in \Omega$ and $y \in \Omega$, define

$$
F_{x, t}(y):=t^{n}\left(1+\frac{|x-y|}{t}\right)^{n+1} K_{t^{2}}(x, y) .
$$

It was proved in [8, p. 156] that $F_{x, t}$ can be extended to a bounded Hölder continuous function on $\mathbb{R}^{n}$ with the Hölder index $\widetilde{\mu} \in(0, \mu)$. We denote this extension by $\widetilde{F}_{x, t}$. For all $t \in\left(0, R_{0}\right], x \in \Omega$ and $y \in \mathbb{R}^{n}$, define

$$
\widetilde{K}_{t^{2}}(x, y):=t^{n}\left(1+\frac{|x-y|}{t}\right)^{-(n+1)} \widetilde{F}_{x, t}(y) .
$$

It was also proved in [8, p. 157] that $\widetilde{K}_{t^{2}}$ satisfies (2.8) and (2.9) with $\mu$ replaced by any $\widetilde{\mu} \in(0, \mu)$. Obviously, for all $t \in(0, \infty)$ and all $x, y \in \Omega$,

$$
K_{t^{2}}(x, y)=\widetilde{K}_{t^{2}}(x, y) .
$$

By Lemma 3.7 and an argument in 86, p. 43], we know that there exist $\left\{\lambda_{i}\right\}_{i} \subset \mathbb{C}$ and a sequence $\left\{a_{i}\right\}_{i}$ of $(\rho, q, 0)_{\omega}$-atoms, with $q \in\left(q_{\omega}, \infty\right]$, such that

$$
\tilde{f}=\sum_{i} \lambda_{i} a_{i}
$$

in $L^{2}\left(\mathbb{R}^{n}\right)$. Thus, for all $t \in\left(0, R_{0}\right]$ and $x \in \Omega$,

$$
\begin{aligned}
e^{-t^{2} L}(f)(x) & =\int_{\Omega} K_{t^{2}}(x, y) f(y) d y \\
& =\int_{\mathbb{R}^{n}} \widetilde{K}_{t^{2}}(x, y) \tilde{f}(y) d y=\sum_{i} \int_{\mathbb{R}^{n}} \lambda_{i} \widetilde{K}_{t^{2}}(x, y) a_{i}(y) d y,
\end{aligned}
$$

which implies that

$$
\mathcal{N}_{h}^{\text {loc }, R_{0}}(f)(x) \leq \sum_{i} \mathcal{N}_{h}^{\text {loc }, R_{0}}\left(\lambda_{i} a_{i}\right)(x)
$$

The remainder of the proof is similar to that of Proposition 3.4(i). We omit the details. This finishes the proof of Proposition 3.4

To show Theorem 1.4, we need the following key proposition.

Proposition 3.8. Let $\Phi, \Omega, \omega$ and $L$ be as in Proposition 3.4, and $R_{0} \in(0, \infty)$. Then there exists a positive constant $C$ such that for all $f \in L^{2}(\Omega)$ satisfying

$$
\begin{gathered}
\left\|\mathcal{N}_{h}^{\text {loc, } 2 R_{0}}(f)\right\|_{L_{\omega}^{\Phi}(\Omega)}<\infty \\
\left\|\widetilde{S}_{h, R_{0}}^{\text {loc }}(f)\right\|_{L_{\omega}^{\Phi}(\Omega)} \leq C\left\|\mathcal{N}_{h}^{\text {loc, } 2 R_{0}}(f)\right\|_{L_{\omega}^{\Phi}(\Omega)} .
\end{gathered}
$$


To show Proposition 3.8, we need the following Lemmas 3.9 through 3.11 .

In [8, p. 183], Auscher and Russ proved the following geometric property of strongly Lipschitz domains, which plays an important role in this paper.

Lemma 3.9. Let $\Omega$ be a strongly Lipschitz domain of $\mathbb{R}^{n}$. Then there exists a constant $C \in(0,1]$ such that for all cubes $Q$ centered in $\Omega$ with

$$
l(Q) \in(0, \infty) \cap(0,2 \operatorname{diam}(\Omega)],
$$

$|Q \cap \Omega| \geq C|Q|$.

In what follows, we denote by $B((z, \tau), r)$ the ball in $\mathbb{R}^{n} \times(0, \infty)$ with center $(z, \tau)$ and radius $r$; namely,

$$
B((z, \tau), r):=\left\{(x, t) \in \mathbb{R}^{n} \times(0, \infty): \max (|x-z|,|t-\tau|)<r\right\} .
$$

Lemma 3.10. Let $\Phi$ satisfy Assumption $(A), \omega \in A_{\infty}\left(\mathbb{R}^{n}\right), R_{0} \in(0, \infty)$ and $L$ be as in (2.7). Let $\Omega$ be either $\mathbb{R}^{n}$ or a strongly Lipschitz domain of $\mathbb{R}^{n}$. Assume that the semigroup generated by $L$ has the Gaussian property $\left(G_{1}\right)$. Then there exist $\epsilon_{0} \in(0, \infty)$ and a positive constant $C$ such that for all $\gamma \in(0,1], \lambda \in(0, \infty)$, $\epsilon, R \in\left(0, R_{0}\right)$ with $\epsilon<R$ and $f \in L^{2}(\Omega)$ satisfying $\left\|\mathcal{N}_{h}^{\text {loc, } 2 R_{0}}(f)\right\|_{L_{\omega}^{\Phi}(\Omega)}<\infty$,

$$
\begin{aligned}
& \omega\left(\left\{x \in \Omega: \widetilde{S}_{h, R_{0}}^{\epsilon, R, \frac{1}{20}}(f)(x)>2 \lambda, \mathcal{N}_{h}^{\text {loc }, 2 R_{0}}(f)(x) \leq \gamma \lambda\right\}\right) \\
& \quad \leq C \gamma^{\epsilon_{0}} \omega\left(\left\{x \in \Omega: \widetilde{S}_{h, R_{0}}^{\epsilon, R}(f)(x)>\lambda\right\}\right) .
\end{aligned}
$$

We point out that in the proof of Proposition 3.8. Lemma 3.10 plays a key role. The inequality (3.14) is usually called the "good- $\lambda$ inequality" concerning the maximal function $\mathcal{N}_{h}^{\text {loc, } 2 R_{0}}(f)$ and the truncated area functions $\widetilde{S}_{h, R_{0}}^{\epsilon, R, \frac{1}{20}}(f)$ and $\widetilde{S}_{h, R_{0}}^{\epsilon, R, \frac{1}{2}}(f)$.

Proof of Lemma 3.10. To prove this lemma, we borrow some ideas from [7] and [8]. Fix $0<\epsilon<R<R_{0}, \gamma \in(0,1]$ and $\lambda \in(0, \infty)$. Let $f \in L^{2}(\Omega)$ satisfy

$$
\left\|\mathcal{N}_{h}^{\text {loc, } 2 R_{0}}(f)\right\|_{L_{\omega}^{\Phi}(\Omega)}<\infty
$$

and

$$
O:=\left\{x \in \Omega: \widetilde{S}_{h, R_{0}}^{\epsilon, \frac{1}{2}}(f)(x)>\lambda\right\} .
$$

It is easy to show that $O$ is an open subset of $\Omega$.

Now we show (3.14) by considering the following two cases for $O$.

Case 1) $O \neq \Omega$. In this case, let

$$
O=\bigcup_{k}\left(Q_{k} \cap \Omega\right)
$$

be the Whitney decomposition of $O$, where $\left\{Q_{k}\right\}_{k}$ are dyadic cubes of $\mathbb{R}^{n}$ with disjoint interiors and $2 Q_{k} \cap \Omega \subset O \subset \Omega$, but

$$
4 Q_{k} \cap \Omega \cap(\Omega \backslash O) \neq \emptyset \text {. }
$$


To show (3.14), by (3.15) and the disjoint property of $\left\{Q_{k}\right\}_{k}$, it suffices to show that for all $k$,

$$
\begin{aligned}
& \omega\left(\left\{x \in Q_{k} \cap \Omega: \widetilde{S}_{h, R_{0}}^{\epsilon, R} \frac{1}{20}(f)(x)>2 \lambda, \mathcal{N}_{h}^{\text {loc }, 2 R_{0}}(f)(x) \leq \gamma \lambda\right\}\right) \\
& \quad \lesssim \gamma^{\epsilon_{0}} \omega\left(Q_{k} \cap \Omega\right) .
\end{aligned}
$$

From now on, we fix $k$ and denote by $l_{k}$ the sidelength of $Q_{k}$.

If $x \in Q_{k} \cap \Omega$, then

$$
\widetilde{S}_{h, R_{0}}^{\max \left\{10 l_{k}, \epsilon\right\}, R, \frac{1}{20}}(f)(x) \leq \lambda .
$$

Indeed, pick $x_{k} \in 4 Q_{k} \cap \Omega$ with $x_{k} \notin O$. For any $(y, t) \in \Omega \times\left(0, R_{0}\right]$, if $|x-y|<\frac{t}{20}$ and $t \geq \max \left\{10 l_{k}, \epsilon\right\}$, then

$$
\left|x_{k}-y\right| \leq\left|x_{k}-x\right|+|x-y|<4 l_{k}+\frac{t}{20}<\frac{t}{2},
$$

which implies that

$$
\Gamma_{\frac{1}{20}}^{\max \left\{10 l_{k}, \epsilon\right\}, R}(x) \subset \Gamma_{\frac{1}{2}}^{\max \left\{10 l_{k}, \epsilon\right\}, R}\left(x_{k}\right) .
$$

By this, we obtain that

$$
\widetilde{S}_{h, R_{0}}^{\max \left\{10 l_{k}, \epsilon\right\}, R, \frac{1}{20}}(f)(x) \leq \widetilde{S}_{h, R_{0}}^{\max \left\{10 l_{k}, \epsilon\right\}, R, \frac{1}{2}}(f)\left(x_{k}\right) \leq \lambda .
$$

Thus, the claim (3.17) holds.

If $\epsilon \geq 10 l_{k}$, by (3.17), we see that (3.16) trivially holds. If $\epsilon<10 l_{k}$, to show (3.16), by the fact that

$$
\widetilde{S}_{h, R_{0}}^{\epsilon, R, \frac{1}{20}}(f) \leq \widetilde{S}_{h, R_{0}}^{\epsilon, 10 l_{k}, \frac{1}{20}}(f)+\widetilde{S}_{h, R_{0}}^{10 l_{k}, R, \frac{1}{20}}(f)
$$

and (3.17), it remains to show that

$$
\omega\left(\left\{x \in Q_{k} \cap F: g(x)>\lambda\right\}\right) \lesssim \gamma^{\epsilon_{0}} \omega\left(Q_{k} \cap \Omega\right),
$$

where $g:=\widetilde{S}_{h, R_{0}}^{\epsilon, 10 l_{k}, \frac{1}{20}}(f)$ and

$$
F:=\left\{x \in \Omega: \mathcal{N}_{h}^{\text {loc }, 2 R_{0}}(f)(x) \leq \gamma \lambda\right\} .
$$

Now we prove (3.18). Similar to the proof of [87, (3.18)], we have

$$
\left|\left\{x \in \Omega \cap Q_{k}: g(x)>\lambda\right\}\right| \lesssim \gamma^{2}\left|Q_{k} \cap \Omega\right| .
$$

By $\omega \in A_{\infty}\left(\mathbb{R}^{n}\right)$, Lemma 2.2 (vi), we know that there exists $r \in(1, \infty]$ such that $\omega \in R H_{r}\left(\mathbb{R}^{n}\right)$, which, together with Lemma 2.2(v), Lemma 3.9 and (3.19), implies that

$$
\begin{aligned}
\frac{\omega\left(\left\{x \in Q_{k} \cap F: g(x)>\lambda\right\}\right)}{\omega\left(Q_{k}\right)} & \lesssim\left(\frac{\left|\left\{x \in Q_{k} \cap F: g(x)>\lambda\right\}\right|}{\left|Q_{k}\right|}\right)^{\frac{r-1}{r}} \\
& \lesssim\left(\frac{\gamma^{2}\left|Q_{k} \cap \Omega\right|}{\left|Q_{k}\right|}\right)^{\frac{r-1}{r}} \sim \gamma^{\frac{2(r-1)}{r}} .
\end{aligned}
$$

Take $\epsilon_{0}:=2(r-1) / r$. Then from (3.20) and Lemmas 3.9 and $2.2($ iii), we deduce that

$$
\omega\left(\left\{x \in Q_{k} \cap F: g(x)>\lambda\right\}\right) \lesssim \gamma^{\epsilon_{0}} \omega\left(Q_{k}\right) \sim \gamma^{\epsilon_{0}} \omega\left(Q_{k} \cap \Omega\right),
$$

which implies that (3.18) holds. Thus, (3.14) holds in the case that $O \neq \Omega$. 
Case 2) $O=\Omega$. In this case, similar to the proof of [87, Lemma 3.5], we know that $\Omega$ is bounded.

Also, similar to the proof of [87, Lemma 3.5], we know that there exists a positive constant $C_{1}$ such that for all $R \in\left(R_{0} / 2, R_{0}\right)$ and $x \in \Omega$,

$$
\widetilde{S}_{h, R_{0}}^{R_{0} / 2, R, \frac{1}{20}}(f)(x) \leq C_{1} \mathcal{N}_{h}^{\text {loc, } 2 R_{0}}(f)(x) .
$$

Now we continue the proof of Lemma 3.10 by using (3.21). Without loss of generality, we may assume that $R \geq R_{0} / 2$. Otherwise, we replace $R$ just by $R_{0} / 2$ in (3.15). If $\gamma \geq \frac{1}{C_{1}}$, then

$$
\begin{aligned}
& \omega\left(\left\{x \in \Omega: \widetilde{S}_{h, R_{0}}^{\epsilon, R} \frac{1}{20}(f)(x)>2 \lambda, \mathcal{N}_{h}^{\text {loc }, 2 R_{0}}(f)(x) \leq \gamma \lambda\right\}\right) \\
& \quad \leq \omega(\Omega) \leq C_{1}^{\epsilon_{0}} \gamma^{\epsilon_{0}} \omega(O) \lesssim \gamma^{\epsilon_{0}} \omega(O),
\end{aligned}
$$

which shows (3.14) in the case that $O=\Omega$ and $\gamma \geq \frac{1}{C_{1}}$.

If $\gamma<\frac{1}{C_{1}}$, by the fact that $\mathcal{N}_{h}^{\text {loc, } 2 R_{0}}(f)(x) \leq \gamma \lambda$ for all $x \in F$ and (3.21), we conclude that for any $R \geq R_{0} / 2$ and $x \in F$,

$$
\widetilde{S}_{h, R_{0}}^{R_{0} / 2, R, \frac{1}{20}}(f)(x) \leq C_{1} \mathcal{N}_{h}^{\text {loc }, 2 R_{0}}(f)(x)<\frac{1}{\gamma} \gamma \lambda=\lambda,
$$

which implies that

$$
\begin{aligned}
& \left\{x \in \Omega: \widetilde{S}_{h, R_{0}}^{\epsilon, R, \frac{1}{20}}(f)(x)>2 \lambda, \mathcal{N}_{h}^{\text {loc }, 2 R_{0}}(f)(x) \leq \gamma \lambda\right\} \\
& \quad \subset\left\{x \in \Omega: \widetilde{S}_{h, R_{0}}^{\epsilon, R_{0} / 2, \frac{1}{20}}(f)(x)>\lambda, \mathcal{N}_{h}^{\text {loc }, 2 R_{0}}(f)(x) \leq \gamma \lambda\right\} .
\end{aligned}
$$

Thus, to finish the proof of (3.14) in this case, it suffices to show that

$$
\left|\left\{x \in \Omega: \widetilde{S}_{h, R_{0}}^{\epsilon, R_{0} / 2, \frac{1}{20}}(f)(x)>\lambda, \mathcal{N}_{h}^{\text {loc, } 2 R_{0}}(f)(x) \leq \gamma \lambda\right\}\right| \lesssim \gamma^{2}|O| .
$$

Indeed, if (3.23) holds, by Lemma 3.9 and the assumption that $\Omega$ is bounded, we know that there exists a cube $Q_{0} \subset \mathbb{R}^{n}$ such that $\Omega \subset Q_{0}$ and $\left|Q_{0}\right| \sim|\Omega|$. From this, $O=\Omega$, (3.22), (3.23), and (iii) and (v) of Lemma 2.2 we infer that

$$
\begin{aligned}
& \frac{\omega\left(\left\{x \in \Omega: \widetilde{S}_{h, R_{0}}^{\epsilon, R, \frac{1}{20}}(f)(x)>2 \lambda, \mathcal{N}_{h}^{\text {loc }, 2 R_{0}}(f)(x) \leq \gamma \lambda\right\}\right)}{\omega\left(Q_{0}\right)} \\
& \leq \frac{\omega\left(\left\{x \in \Omega: \widetilde{S}_{h, R_{0}}^{\epsilon, R_{0} / 2, \frac{1}{20}}(f)(x)>\lambda, \mathcal{N}_{h}^{\text {loc }, 2 R_{0}}(f)(x) \leq \gamma \lambda\right\}\right)}{\omega\left(Q_{0}\right)} \\
& \quad \lesssim\left\{\frac{\left|\left\{x \in \Omega: \widetilde{S}_{h, R_{0}}^{\epsilon, R_{0} / 2, \frac{1}{20}}(f)(x)>\lambda, \mathcal{N}_{h}^{\text {loc }, 2 R_{0}}(f)(x) \leq \gamma \lambda\right\}\right|}{\left|Q_{0}\right|}\right\}^{\frac{r-1}{r}} \\
& \quad \lesssim\left\{\frac{\gamma^{2}|\Omega|}{\left|Q_{0}\right|}\right\}^{\frac{r-1}{r}} \sim \gamma^{\epsilon_{0}} .
\end{aligned}
$$

By this, the fact that $\left|Q_{0}\right| \sim|\Omega|$, (iii) and (v) of Lemma 2.2 and $O=\Omega$, we conclude that

$\omega\left(\left\{x \in \Omega: \widetilde{S}_{h, R_{0}}^{\epsilon, R, \frac{1}{20}}(f)(x)>2 \lambda, \mathcal{N}_{h}^{\text {loc }, 2 R_{0}}(f)(x) \leq \gamma \lambda\right\}\right) \lesssim \gamma^{\epsilon_{0}} \omega\left(Q_{0}\right) \sim \gamma^{\epsilon_{0}} \omega(O)$,

which shows (3.14) in the case that $O=\Omega$ and $\gamma<\frac{1}{C_{1}}$. 
Finally, we point out that the proof of (3.23) is similar to that of (3.19) with $10 l_{k}$ and $Q_{k} \cap F$, respectively, replaced by $R_{0} / 2$ and $\Omega$. We omit the details, which completes the proof of Lemma 3.10 .

Lemma 3.11. Let $\Phi, \Omega$ and $L$ be as in Proposition 3.4. Let $\omega \in A_{\infty}\left(\mathbb{R}^{n}\right)$ and $R_{0} \in(0, \infty)$. For all $\alpha, \beta \in(0, \infty), 0<\epsilon<R<R_{0}$ and all $f \in L^{2}(\Omega)$,

$$
\int_{\Omega} \Phi\left(\widetilde{S}_{h, R_{0}}^{\epsilon, R}(f)(x)\right) \omega(x) d x \sim \int_{\Omega} \Phi\left(\widetilde{S}_{h, R_{0}}^{\epsilon, R}(f)(x)\right) \omega(x) d x
$$

where the implicit constants are independent of $\epsilon, R$ and $f$.

The proof of Lemma 3.11 is similar to that of 20, Proposition 4]. We omit the details.

Now we show Proposition 3.8 by virtue of Lemmas 3.10 and 3.11

Proof of Proposition 3.8. Let $f \in L^{2}(\Omega)$ satisfy $\left\|\mathcal{N}_{h}^{\text {loc, } 2 R_{0}}(f)\right\|_{L_{\omega}^{\Phi}(\Omega)}<\infty$. By the upper type 1 and the lower type $p_{0}$ properties of $\Phi$, where $p_{0} \in\left(0, p_{\Phi}^{-}\right)$, we know that for all $t \in(0, \infty)$,

$$
\Phi(t) \sim \int_{0}^{t} \frac{\Phi(s)}{s} d s .
$$

From this, Fubini's theorem and Lemma 3.10, it follows that for all $\epsilon, R \in\left(0, R_{0}\right]$ with $\epsilon<R$ and $\gamma \in(0,1]$,

$$
\begin{aligned}
\int_{\Omega} & \Phi\left(\widetilde{S}_{h, R_{0}}^{\epsilon, R, \frac{1}{20}}(f)(x)\right) \omega(x) d x \\
& \sim \int_{\Omega}\left\{\int_{0}^{\widetilde{S}_{h, R_{0}}^{\epsilon, R}(f)(x)} \frac{\Phi(t)}{t} d t\right\} \omega(x) d x \sim \int_{0}^{\infty} \frac{\Phi(t)}{t} \sigma_{\widetilde{S}_{h, R_{0}}^{\epsilon, R, \frac{1}{20}}(f)}(t) d t \\
& \lesssim \int_{0}^{\infty} \frac{\Phi(t)}{t} \sigma_{\mathcal{N}_{h}^{\text {loc, } 2 R_{0}}(f)}(\gamma t) d t+\gamma^{\epsilon} \int_{0}^{\infty} \frac{\Phi(t)}{t} \sigma_{\widetilde{S}_{h, R_{0}}^{\epsilon, R, \frac{1}{2}}(f)}(t / 2) d t \\
& \lesssim \frac{1}{\gamma} \int_{0}^{\infty} \frac{\Phi(t)}{t} \sigma_{\mathcal{N}_{h}^{\text {loc }, 2 R_{0}}(f)}(t) d t+\gamma^{\epsilon_{0}} \int_{0}^{\infty} \frac{\Phi(t)}{t} \sigma_{\widetilde{S}_{h, R_{0}}^{\epsilon, \frac{1}{2}}(f)}(t) d t \\
& \sim \frac{1}{\gamma} \int_{\Omega} \Phi\left(\mathcal{N}_{h}^{\text {loc, } 2 R_{0}}(f)(x)\right) \omega(x) d x+\gamma^{\epsilon} \int_{\Omega} \Phi\left(\widetilde{S}_{h, R_{0}}^{\epsilon, R, \frac{1}{2}}(f)(x)\right) \omega(x) d x
\end{aligned}
$$

where

$$
\sigma_{\widetilde{S}_{h, R_{0}}^{\epsilon, R, \frac{1}{20}(f)}}(t):=\omega\left(\left\{x \in \Omega: \widetilde{S}_{h, R_{0}}^{\epsilon, R, \frac{1}{20}}(f)(x)>t\right\}\right)
$$

and $\epsilon_{0}$ is as in Lemma 3.10 .

Furthermore, by Lemma 3.11, (3.24) and $\widetilde{S}_{h, R_{0}}^{\epsilon, R, \frac{1}{2}}(f) \leq \widetilde{S}_{h, R_{0}}^{\epsilon, R}(f)$, we know that for all $\epsilon, R \in\left(0, R_{0}\right]$ with $\epsilon<R$ and $\gamma \in(0,1]$,

$$
\begin{aligned}
\int_{\Omega} \Phi\left(\widetilde{S}_{h, R_{0}}^{\epsilon, R}(f)(x)\right) \omega(x) d x & \sim \int_{\Omega} \Phi\left(\widetilde{S}_{h, R_{0}}^{\epsilon, R} \frac{1}{20}(f)(x)\right) \omega(x) d x \\
\lesssim & \frac{1}{\gamma} \int_{\Omega} \Phi\left(\mathcal{N}_{h}^{\text {loc, } 2 R_{0}}(f)(x)\right) \omega(x) d x \\
& +\gamma^{\epsilon_{0}} \int_{\Omega} \Phi\left(\widetilde{S}_{h, R_{0}}^{\epsilon, R}(f)(x)\right) \omega(x) d x
\end{aligned}
$$


which, together with the facts that for all $\lambda \in(0, \infty)$,

$$
\widetilde{S}_{h, R_{0}}^{\epsilon, R}(f / \lambda)=\widetilde{S}_{h, R_{0}}^{\epsilon, R}(f) / \lambda \quad \text { and } \quad \mathcal{N}_{h}^{\text {loc, } 2 R_{0}}(f / \lambda)=\mathcal{N}_{h}^{\text {loc, } 2 R_{0}}(f) / \lambda,
$$

implies that there exists a positive constant $C_{2}$ such that

$$
\begin{aligned}
\int_{\Omega} \Phi & \left(\frac{\widetilde{S}_{h, R_{0}}^{\epsilon, R}(f)(x)}{\lambda}\right) \omega(x) d x \\
\leq & C_{2}\left\{\frac{1}{\gamma} \int_{\Omega} \Phi\left(\frac{\mathcal{N}_{h}^{\text {loc }, 2 R_{0}}(f)(x)}{\lambda}\right) \omega(x) d x\right. \\
& \left.+\gamma^{\epsilon_{0}} \int_{\Omega} \Phi\left(\frac{\widetilde{S}_{h, R_{0}}^{\epsilon, R}(f)(x)}{\lambda}\right) \omega(x) d x\right\} .
\end{aligned}
$$

Take $\gamma \in(0,1]$ such that $C_{2} \gamma^{\epsilon_{0}}=\frac{1}{2}$. Then from (3.25), it follows that for all $\lambda \in(0, \infty)$,

$$
\int_{\Omega} \Phi\left(\frac{\widetilde{S}_{h, R_{0}}^{\epsilon, R}(f)(x)}{\lambda}\right) \omega(x) d x \lesssim \int_{\Omega} \Phi\left(\frac{\mathcal{N}_{h}^{\text {loc }, 2 R_{0}}(f)(x)}{\lambda}\right) \omega(x) d x .
$$

By the Fatou lemma and letting $\epsilon \rightarrow 0$ and $R \rightarrow R_{0}$, we see that for any $\lambda \in(0, \infty)$,

$$
\int_{\Omega} \Phi\left(\frac{\widetilde{S}_{h, R_{0}}^{\text {loc }}(f)(x)}{\lambda}\right) \omega(x) d x \lesssim \int_{\Omega} \Phi\left(\frac{\mathcal{N}_{h}^{\text {loc }, 2 R_{0}}(f)(x)}{\lambda}\right) \omega(x) d x,
$$

which implies that

$$
\left\|\widetilde{S}_{h, R_{0}}^{\text {loc }}(f)\right\|_{L_{\omega}^{\Phi}(\Omega)} \lesssim\left\|\mathcal{N}_{h}^{\text {loc, } 2 R_{0}}(f)\right\|_{L_{\omega}^{\Phi}(\Omega)} .
$$

This finishes the proof of Proposition 3.8

Proposition 3.12. Let $\Phi, \Omega, \omega$ and $L$ be as in Proposition 3.4 and $R_{0} \in(0, \infty)$. Then there exists a positive constant $C$ such that for all $f \in L^{2}(\Omega)$,

$$
\left\|S_{h, R_{0}}^{\text {loc }}(f)\right\|_{L_{\omega}^{\Phi}(\Omega)} \leq C\left\|\widetilde{S}_{h, R_{0}}^{\text {loc }}(f)\right\|_{L_{\omega}^{\Phi}(\Omega)} .
$$

Proof. Let $f \in L^{2}(\Omega), x \in \Omega$ and $p_{0} \in\left(0, p_{\Phi}^{-}\right)$. Then by the definition of $p_{\Phi}^{-}$, we know that $\Phi$ is of lower type $p_{0}$. Similar to the proof of [87, (3.38)], we see that there exists a positive constant $C_{3}$, independent of $f$ and $x$, such that for all $\varepsilon \in(0,1)$

$$
S_{h, R_{0}}^{\mathrm{loc}}(f)(x) \leq \frac{C_{3}}{\varepsilon} \widetilde{S}_{h, R_{0}}^{\mathrm{loc}, \frac{3}{2}}(f)(x)+\varepsilon S_{h, R_{0}}^{\mathrm{loc}, 2}(f)(x) .
$$

Also, similar to the proof of Lemma 3.11, we conclude that there exists a positive constant $C_{4}$ such that for all $g \in L^{2}(\Omega)$,

$$
\int_{\Omega} \Phi\left(S_{h, R_{0}}^{\mathrm{loc}, 2}(g)(y)\right) \omega(y) d y \leq C_{4} \int_{\Omega} \Phi\left(S_{h, R_{0}}^{\mathrm{loc}}(g)(y)\right) \omega(y) d y .
$$

From this, (3.26), the lower type $p_{0}$ and the upper type 1 properties of $\Phi$, it follows that there exists a positive constant $\widetilde{C}$ such that

$$
\int_{\Omega} \Phi\left(S_{h, R_{0}}^{\mathrm{loc}}(f)(x)\right) \omega(x) d x
$$




$$
\begin{aligned}
\leq & \int_{\Omega} \Phi\left(\frac{C_{3}}{\varepsilon} \widetilde{S}_{h, R_{0}}^{\mathrm{loc}, \frac{3}{2}}(f)(x)\right) \omega(x) d x+\int_{\Omega} \Phi\left(\varepsilon S_{h, R_{0}}^{\mathrm{loc}, 2}(f)(x)\right) \omega(x) d x \\
\leq & \widetilde{C}\left\{\frac{C_{3}}{\varepsilon} \int_{\Omega} \Phi\left(\widetilde{S}_{h, R_{0}}^{\frac{3}{2}}(f)(x)\right) \omega(x) d x\right. \\
& \left.+C_{4} \varepsilon^{p_{0}} \int_{\Omega} \Phi\left(S_{h, R_{0}}^{\mathrm{loc}}(f)(x)\right) \omega(x) d x\right\} .
\end{aligned}
$$

Take $\varepsilon \in(0,1)$ small enough such that $\widetilde{C} C_{4} \varepsilon^{p_{0}} \leq \frac{1}{2}$. By this, (3.27) and Lemma 3.11, we see that

$$
\int_{\Omega} \Phi\left(S_{h, R_{0}}^{\mathrm{loc}}(f)(x)\right) \omega(x) d x \lesssim \int_{\Omega} \Phi\left(\widetilde{S}_{h, R_{0}}^{\mathrm{loc}}(f)(x)\right) \omega(x) d x
$$

which, together with the facts that for all $\lambda \in(0, \infty)$,

$$
S_{h, R_{0}}^{\text {loc }}(f / \lambda)=S_{h, R_{0}}^{\text {loc }}(f) / \lambda \quad \text { and } \quad \widetilde{S}_{h, R_{0}}^{\text {loc }}(f / \lambda)=\widetilde{S}_{h, R_{0}}^{\text {loc }}(f) / \lambda,
$$

implies that

$$
\int_{\Omega} \Phi\left(\frac{S_{h, R_{0}}^{\mathrm{loc}}(f)(x)}{\lambda}\right) \omega(x) d x \lesssim \int_{\Omega} \Phi\left(\frac{\widetilde{S}_{h, R_{0}}^{\mathrm{loc}}(f)(x)}{\lambda}\right) \omega(x) d x .
$$

From this, the desired conclusion follows, which completes the proof of Proposition 3.12 .

To complete the proof of Theorem 1.4, we need the following key proposition.

Proposition 3.13. Let $\Phi, \Omega, L, \omega, p_{\Phi}^{-}, p_{\Phi}^{+}$and $r_{\omega}$ be as in Theorem 1.4, and $R_{0} \in(0, \infty)$. Assume that the semigroup generated by $L$ has the Gaussian property $\left(G_{1}\right)$.

(i) If $\Omega:=\mathbb{R}^{n}$, then there exists a positive constant $C$ such that for all $f \in L^{2}\left(\mathbb{R}^{n}\right)$ satisfying $\left\|S_{h, R_{0}}^{\text {loc }}(f)\right\|_{L_{\omega}^{\Phi}\left(\mathbb{R}^{n}\right)}<\infty$,

$$
\begin{aligned}
\|f\|_{h_{\omega}^{\Phi}\left(\mathbb{R}^{n}\right)} \leq C & {\left[\left\|S_{h, R_{0}}^{\text {loc }}(f)\right\|_{L_{\omega}^{\Phi}\left(\mathbb{R}^{n}\right)}\right.} \\
& \left.+\inf \left\{\lambda \in(0, \infty): \sum_{Q_{k} \in \mathcal{Q}} \omega\left(Q_{k}\right) \Phi\left(\frac{m_{Q_{k}}\left(\left|e^{-R_{0}^{2} L}(f)\right|\right)}{\lambda}\right) \leq 1\right\}\right] .
\end{aligned}
$$

(ii) Under $D B C$, if $\Omega^{\complement}$ is unbounded, then there exists a positive constant $C$ such that for all $f \in L^{2}(\Omega)$ satisfying $\left\|S_{h, R_{0}}^{\text {loc }}(f)\right\|_{L_{\omega}^{\Phi}(\Omega)}<\infty$,

$$
\begin{aligned}
\|f\|_{h_{\omega, r}^{\Phi}(\Omega)} \leq C\left[\left\|S_{h, R_{0}}^{\mathrm{loc}}(f)\right\|_{L_{\omega}^{\Phi}(\Omega)}+\inf \{\lambda \in(0, \infty):\right. \\
\left.\qquad \sum_{\widetilde{Q}_{k} \in \mathcal{Q}_{\Omega}} \omega\left(\widetilde{Q}_{k} \cap \Omega\right) \Phi\left(\frac{m_{\widetilde{Q}_{k} \cap \Omega}\left(\left|e^{-R_{0}^{2} L}(f)\right|\right)}{\lambda}\right) \leq 1\right\} .
\end{aligned}
$$


(iii) Under $N B C$, there exists a positive constant $C$ such that for all $f \in L^{2}(\Omega)$ satisfying $\left\|S_{h, R_{0}}^{\text {loc }}(f)\right\|_{L_{\omega}^{\Phi}(\Omega)}<\infty$,

$$
\begin{aligned}
\|f\|_{h_{\omega, r}^{\Phi}(\Omega)} \leq C\left[\left\|S_{h, R_{0}}^{\mathrm{loc}}(f)\right\|_{L_{\omega}^{\Phi}(\Omega)}+\inf \{\lambda \in(0, \infty):\right. \\
\left.\qquad \sum_{\widetilde{Q}_{k} \in \mathcal{Q}_{\Omega}} \omega\left(\widetilde{Q}_{k} \cap \Omega\right) \Phi\left(\frac{m_{\widetilde{Q}_{k} \cap \Omega}\left(\left|e^{-R_{0}^{2} L}(f)\right|\right)}{\lambda}\right) \leq 1\right\} .
\end{aligned}
$$

Let $\Omega$ be either $\mathbb{R}^{n}$ or a strongly Lipschitz domain of $\mathbb{R}^{n}$. To show Proposition 3.13. we need the atomic decomposition of functions in the local tent space on $\Omega$. Now we recall some definitions and notation about the tent space, which was initially introduced by Coifman, Meyer and Stein $\left[20\right.$ on $\mathbb{R}^{n}$, and then generalized by Russ 73 to spaces of homogeneous type in the sense of Coifman and Weiss 21, 22. Recently, Carbonaro, McIntosh and Morris 14 developed the theory of the local tent space on locally doubling metric measure spaces. Recall that it is well known that the strongly Lipschitz domain $\Omega$ is a space of homogeneous type.

Let $R_{0} \in(0, \infty)$ and $\omega \in A_{\infty}\left(\mathbb{R}^{n}\right)$. For all measurable functions $g$ on $\Omega \times\left(0, R_{0}\right]$ and $x \in \Omega$, define

$$
\mathcal{A}^{\mathrm{loc}, R_{0}}(g)(x):=\left[\int_{\Gamma^{R_{0}(x)}}|g(x, t)|^{2} \frac{d y}{|Q(x, t) \cap \Omega|} \frac{d t}{t}\right]^{\frac{1}{2}},
$$

where

$$
\Gamma^{R_{0}}(x):=\left\{(y, t) \in \Omega \times\left(0, R_{0}\right]:|y-x|<t\right\} .
$$

In what follows, we denote by $T_{\Phi, \omega}^{\text {loc, } R_{0}}(\Omega)$ the space of all measurable functions $g$ on $\Omega \times\left(0, R_{0}\right]$ such that $\mathcal{A}^{\text {loc, } R_{0}}(g) \in L_{\omega}^{\Phi}(\Omega)$ and for any $g \in T_{\Phi, \omega}^{\text {loc, } R_{0}}(\Omega)$, define its norm by

$$
\begin{aligned}
\|g\|_{T_{\Phi, \omega}^{\text {loc, } R_{0}}(\Omega)} & :=\left\|\mathcal{A}^{\mathrm{loc}, R_{0}}(g)\right\|_{L_{\omega}^{\Phi}(\Omega)} \\
& :=\inf \left\{\lambda \in(0, \infty): \int_{\Omega} \Phi\left(\frac{\mathcal{A}^{\mathrm{loc}, R_{0}}(g)(x)}{\lambda}\right) \omega(x) d x \leq 1\right\} .
\end{aligned}
$$

A function $a$ on $\Omega \times\left(0, R_{0}\right]$ is called a $T_{\Phi, \omega}^{\text {loc, } R_{0}}(\Omega)$-atom if

(i) there exists a cube $Q:=Q\left(x_{Q}, l(Q)\right) \subset \mathbb{R}^{n}$ with $x_{Q} \in \Omega$ and

$$
l(Q) \in(0, \infty) \cap(0, \operatorname{diam}(\Omega)]
$$

such that $\operatorname{supp}(a) \subset \widehat{Q \cap \Omega}$, where and in what follows,

$$
\widehat{Q \cap \Omega}:=\left\{(y, t) \in \Omega \times\left(0, R_{0}\right]:\left|y-x_{Q}\right|<\frac{l(Q)}{2}-t\right\} ;
$$

(ii)

$$
\|a\|_{T_{2}^{2}\left(\Omega \times\left(0, R_{0}\right]\right)}^{2}:=\int_{\widehat{Q \cap \Omega}}|a(y, t)|^{2} \frac{d y d t}{t} \leq|Q \cap \Omega|[\omega(Q \cap \Omega) \rho(\omega(Q \cap \Omega))]^{-2} .
$$

Since $\Phi$ is concave, by Jensen's inequality, it is easy to see that for all $T_{\Phi, \omega}^{\mathrm{loc}, R_{0}}(\Omega)$ atoms $a$, we have

$$
\|a\|_{T_{\Phi, \omega}^{\mathrm{loc}, R_{0}}(\Omega)} \leq 1
$$


see [4]. By a slight modification on the proof of [48, Theorem 3.1], we have the following atomic decomposition for functions in $T_{\Phi, \omega}^{\text {loc, } R_{0}}(\Omega)$. We omit the details.

Lemma 3.14. Let $\Phi$ satisfy Assumption $(A), \Omega$ be either $\mathbb{R}^{n}$ or a strongly Lipschitz domain of $\mathbb{R}^{n}$ and $\omega \in A_{\infty}\left(\mathbb{R}^{n}\right)$. Then for all $f \in T_{\Phi, \omega}^{\text {loc, } R_{0}}(\Omega)$, there exist a sequence $\left\{a_{j}\right\}_{j}$ of $T_{\Phi, \omega}^{\text {loc, } R_{0}}(\Omega)$-atoms and a sequence $\left\{\lambda_{j}\right\}_{j} \subset \mathbb{C}$ such that for almost every $(x, t) \in \Omega \times\left(0, R_{0}\right]$,

$$
f(x, t)=\sum_{j} \lambda_{j} a_{j}(x, t) .
$$

Moreover, there exists a positive constant $C$ such that for all $f \in T_{\Phi, \omega}^{\mathrm{loc}, R_{0}}(\Omega)$,

$$
\begin{aligned}
& \Lambda\left(\left\{\lambda_{j} a_{j}\right\}_{j}\right) \\
& \quad:=\inf \left\{\lambda \in(0, \infty): \sum_{j} \omega\left(Q_{j} \cap \Omega\right) \Phi\left(\frac{\left|\lambda_{j}\right|}{\lambda \omega\left(Q_{j} \cap \Omega\right) \rho\left(\omega\left(Q_{j} \cap \Omega\right)\right)}\right) \leq 1\right\} \\
& \quad \leq C\|f\|_{T_{\Phi, \omega}^{\text {loc, } R_{0}(\Omega)},}
\end{aligned}
$$

where for each $j, Q_{j} \cap \Omega$ appears in the support of $a_{j}$.

In [8, p. 183], Auscher and Russ showed the following property of strongly Lipschitz domains, which plays an important role in the proof of Proposition 3.13 .

Lemma 3.15. Let $\Omega$ be a strongly Lipschitz domain of $\mathbb{R}^{n}$. Then there exists $\gamma_{\Omega} \in(0, \infty)$ such that for any cube $Q$ satisfying $l(Q)<\gamma_{\Omega}$ and $2 Q \subset \Omega$ but $4 Q \cap \partial \Omega \neq \emptyset$, where $\partial \Omega$ denotes the boundary of $\Omega$, there exists a cube $\widetilde{Q} \subset \Omega^{\complement}$ such that $l(\widetilde{Q})=l(Q)$ and the distance from $\widetilde{Q}$ to $Q$ is comparable to $l(Q)$. Furthermore, $\gamma_{\Omega}=\infty$ if $\Omega^{\complement}$ is unbounded.

Now we show Proposition 3.13 by applying Lemmas 3.7, 3.14 and 3.15.

Proof of Proposition 3.13. We first prove Proposition 3.13(i) by borrowing some ideas from the proof of [22, p. 594, Theorem C] (see also [42] and [51]). Let $a$ be a $T_{\Phi, \omega}^{\text {loc, } R_{0}}\left(\mathbb{R}^{n}\right)$-atom, $\operatorname{supp}(a) \subset \widehat{Q}$ with $Q:=Q\left(x_{0}, r_{0}\right)$, and

$$
\alpha:=8 \int_{0}^{R_{0}} t^{2} L e^{-t^{2} L}(a) \frac{d t}{t} .
$$

Set $R_{k}(Q):=2^{k+1} Q \backslash 2^{k} Q$ when $k \in \mathbb{N}$ and $R_{0}(Q):=2 Q$. For $k \in \mathbb{Z}_{+}$, let $\chi_{k}:=\chi_{R_{k}(Q)}, \tilde{\chi}_{k}:=\left|R_{k}(Q)\right|^{-1} \chi_{k}$,

$$
m_{k}:=\int_{R_{k}(Q)} \alpha(x) d x
$$

and $M_{k}:=\alpha \chi_{k}-m_{k} \widetilde{\chi}_{k}$. Then we have

$$
\alpha=\sum_{k=0}^{\infty} M_{k}+\sum_{k=0}^{\infty} m_{k} \tilde{\chi}_{k} .
$$

For $j \in \mathbb{Z}_{+}$, let

$$
N_{j}:=\sum_{k=j}^{\infty} m_{k} .
$$


By [8, Lemma A.5(a)], we see that

$$
\int_{\mathbb{R}^{n}} \alpha(x) d x=0
$$

which, together with (3.29), yields that

$$
\alpha=\sum_{k=0}^{\infty} M_{k}+\sum_{k=0}^{\infty} N_{k+1}\left(\widetilde{\chi}_{k+1}-\tilde{\chi}_{k}\right) .
$$

Obviously, for all $k \in \mathbb{Z}_{+}$,

$$
\int_{\mathbb{R}^{n}} M_{k}(x) d x=0
$$

In what follows, if (2.10) holds with $p_{\Phi}^{+}$for all $t \in[1, \infty)$ and $s \in(0, \infty)$, then we choose $\widetilde{p}_{\Phi}:=p_{\Phi}^{+}$; otherwise, since $\Phi$ is concave, we know $p_{\Phi}^{+}<1$ and we choose $\widetilde{p}_{\Phi} \in\left(p_{\Phi}^{+}, 1\right)$ to be close enough to $p_{\Phi}^{+}$. Then we know that $\Phi$ has the upper type $\widetilde{p}_{\Phi}$ property. From the hypotheses

$$
\frac{q_{\omega}}{p_{\Phi}^{-}}<\frac{n+\mu}{n}, \frac{2 q_{\omega}}{p_{\Phi}^{-}}<\frac{n+1}{n}+\frac{r_{\omega}-1}{p_{\Phi}^{+} r_{\omega}} \text { and } r_{\omega}>\frac{2}{2-q_{\omega}},
$$

we deduce that there exist $p_{0} \in\left(0, p_{\Phi}^{-}\right), r_{0} \in\left(1, r_{\omega}\right)$, and $q_{1}, q_{2}, q_{3} \in\left(q_{\omega}, 2\right)$ such that

$$
\frac{q_{1}}{p_{0}}<\frac{n+\mu}{n}, \frac{2 q_{2}}{p_{0}}<\frac{n+1}{n}+\frac{r_{0}-1}{\widetilde{p}_{\Phi} r_{0}} \text { and } \frac{2}{2-q_{3}}<r_{0} .
$$

Take $q:=\min \left\{q_{1}, q_{2}, q_{3}\right\}$. Then $q \in\left(q_{\omega}, 2\right)$,

$$
\frac{q}{p_{0}}<\frac{n+\mu}{n}, \frac{2 q}{p_{0}}<\frac{n+1}{n}+\frac{r_{0}-1}{\widetilde{p}_{\Phi} r_{0}} \text { and } \frac{2}{2-q}<r_{0} .
$$

By the third inequality in (3.32), we know that $\omega \in R H_{2 /(2-q)}\left(\mathbb{R}^{n}\right)$. Moreover, similar to the proof of [87, (3.51)], we know that

$$
\|\alpha\|_{L^{2}\left(\mathbb{R}^{n}\right)} \lesssim\|a\|_{T_{2}^{2}\left(\mathbb{R}^{n} \times\left(0, R_{0}\right]\right)} .
$$

Then from this, $\omega \in R H_{2 /(2-q)}\left(\mathbb{R}^{n}\right)$, Hölder's inequality, (2.1), (2.3) and Lemma 2.2(iii), we infer that when $k=0$, there exists a positive constant $C_{5}$ such that

$$
\begin{aligned}
\left\|M_{0}\right\|_{L_{\omega}^{q}\left(\mathbb{R}^{n}\right)} \leq & \|\alpha\|_{L_{\omega}^{q}(2 Q)}+\left|m_{0}\right| \frac{[\omega(2 Q)]^{\frac{1}{q}}}{|2 Q|} \\
\leq & \|\alpha\|_{L_{\omega}^{q}(2 Q)}+\left\{\int_{2 Q}|\alpha(x)|^{q} \omega(x) d x\right\}^{\frac{1}{q}} \\
& \times\left\{\int_{2 Q}[\omega(x)]^{-\frac{q^{\prime}}{q}} d x\right\}^{\frac{1}{q^{\prime}}} \frac{[\omega(2 Q)]^{\frac{1}{q}}}{|2 Q|} \\
\lesssim & \|\alpha\|_{L_{\omega}^{q}(2 Q)} \lesssim\|\alpha\|_{L^{2}(2 Q)}\left\{\int_{2 Q}[\omega(x)]^{\frac{2}{2-q}} d x\right\}^{\frac{1}{q}-\frac{1}{2}} \\
\lesssim & \|a\|_{T_{2}^{2}\left(\mathbb{R}^{n} \times\left(0, R_{0}\right]\right)} \frac{[\omega(2 Q)]^{\frac{1}{q}}}{|2 Q|^{\frac{1}{2}}} \leq C_{5}[\omega(2 Q)]^{\frac{1}{q}-1}[\rho(\omega(2 Q))]^{-1}
\end{aligned}
$$


which, together with (3.31) and $\operatorname{supp}\left(M_{0}\right) \subset 2 Q$, implies that $M_{0} / C_{5}$ is a $(\rho, q, 0)_{\omega^{-}}$ atom. When $k \geq 1$, similar to the proof of [87, (3.53)], we know that for all $x \in R_{k}(Q)$,

$$
|\alpha(x)| \lesssim 2^{-k(n+1)}|Q|^{-\frac{1}{2}}\|a\|_{T_{2}^{2}\left(\mathbb{R}^{n} \times\left(0, R_{0}\right]\right)} .
$$

Thus, for all $k \in \mathbb{N}$, from (3.34), Hölder's inequality, (2.1), Lemma 2.2(iii), the upper type $\frac{1}{p_{0}}-1$ property of $\rho$ and the first inequality of (3.32), it follows that there exists a positive constant $C_{6}$ such that

$$
\begin{aligned}
\left\|M_{k}\right\|_{L_{\omega}^{q}\left(\mathbb{R}^{n}\right)} \leq & \|\alpha\|_{L_{\omega}^{q}\left(R_{k}(Q)\right)}+\left|m_{k}\right| \frac{\left[\omega\left(R_{k}(Q)\right)\right]^{\frac{1}{q}}}{\left|R_{k}(Q)\right|} \\
\leq & \|\alpha\|_{L_{\omega}^{q}\left(R_{k}(Q)\right)} \\
& +\|\alpha\|_{L_{\omega}^{q}\left(R_{k}(Q)\right)}\left\{\int_{R_{k}(Q)}[\omega(x)]^{-q^{\prime} / q} d x\right\}^{\frac{1}{q^{\prime}}} \frac{\left[\omega\left(R_{k}(Q)\right)\right]^{\frac{1}{q}}}{\left|R_{k}(Q)\right|} \\
\lesssim & \|\alpha\|_{L_{\omega}^{q}\left(R_{k}(Q)\right)} \lesssim 2^{-k(n+1)}[\omega(Q) \rho(\omega(Q))]^{-1}\left[\omega\left(2^{k+1} Q\right)\right]^{\frac{1}{q}} \\
\lesssim & 2^{-k(n+1)} 2^{k n q}\left[\omega\left(2^{k+1} Q\right)\right]^{\frac{1}{q}-1}\left[\rho\left(2^{-k n q} \omega\left(2^{k+1} Q\right)\right)\right]^{-1} \\
\lesssim & C_{6} 2^{-k n\left(\frac{n+1}{n}-\frac{q}{p_{0}}\right)}\left[\omega\left(2^{k+1} Q\right)\right]^{\frac{1}{q}-1}\left[\rho\left(\omega\left(2^{k+1} Q\right)\right)\right]^{-1},
\end{aligned}
$$

which, together with (3.31) and the fact that for each $k \in \mathbb{N}, \operatorname{supp}\left(M_{k}\right) \subset 2^{k+1} Q$, implies that for each $k \in \mathbb{N}, 2^{k n\left(\frac{n+1}{n}-\frac{q}{p_{0}}\right)} M_{k} / C_{6}$ is a $(\rho, q, 0)_{\omega}$-atom. Moreover, by an argument similar to that used in [87, p. 44], we know that $\sum_{k=0}^{\infty} M_{k}$ converges in $L^{2}\left(\mathbb{R}^{n}\right)$. Let

$$
\lambda_{1, k}:=C_{6} 2^{-k n\left(\frac{n+1}{n}-\frac{q}{p_{0}}\right)} \text { and } a_{1, k}:=2^{k n\left(\frac{n+1}{n}-\frac{q}{p_{0}}\right)} M_{k} / C_{6}
$$

when $k \in \mathbb{N}, \lambda_{1,0}:=C_{5}$ and $a_{1,0}:=M_{0} / C_{5}$. Then $\left\{a_{1, k}\right\}_{k=0}^{\infty}$ is a sequence of $(\rho, q, 0)_{\omega}$-atoms. Furthermore, by the definitions of $\left\{\lambda_{1, k}\right\}_{k=0}^{\infty}$,

$$
\omega \in A_{q}\left(\mathbb{R}^{n}\right) \cap R H_{r_{0}}\left(\mathbb{R}^{n}\right),
$$

(iii) and (v) of Lemma 2.2, the lower type $\frac{1}{\widetilde{p}_{\Phi}}-1$ property of $\rho$ and the second inequality in (3.32), we know that for all $\lambda \in(0, \infty)$,

$$
\begin{aligned}
& \sum_{k=0}^{\infty} \omega\left(2^{k+1} Q\right) \Phi\left(\frac{\left|\lambda_{1, k}\right|}{\lambda \omega\left(2^{k+1} Q\right) \rho\left(\omega\left(2^{k+1} Q\right)\right)}\right) \\
& \quad \lesssim \sum_{k=0}^{\infty} 2^{(k+1) n q} \omega(Q) \Phi\left(\frac{2^{-k n\left(\frac{n+1}{n}-\frac{q}{p_{0}}\right)}}{\lambda \omega\left(2^{k+1} Q\right) \rho\left(\omega\left(2^{k+1} Q\right)\right)}\right) \\
& \quad \lesssim \sum_{k=0}^{\infty} 2^{(k+1) n q} \omega(Q) \Phi\left(\frac{2^{-k n\left(\frac{n+1}{n}-\frac{q}{p_{0}}\right)} 2^{-\frac{k n\left(r_{0}-1\right)}{\tilde{p}_{\Phi} r_{0}}}}{\lambda \omega(Q) \rho(\omega(Q))}\right) \\
& \quad \lesssim \sum_{k=0}^{\infty} 2^{-k n\left[\frac{(n+1) p_{0}}{n}+\frac{p_{0}\left(r_{0}-1\right)}{\tilde{p}_{\Phi} r_{0}}-2 q\right]} \omega(Q) \Phi\left(\frac{1}{\lambda \omega(Q) \rho(\omega(Q))}\right) \\
& \quad \lesssim \omega(Q) \Phi\left(\frac{1}{\lambda \omega(Q) \rho(\omega(Q))}\right),
\end{aligned}
$$


which, together with Lemma 3.7 implies that

$$
\sum_{k=0}^{\infty} M_{k} \in h_{\omega}^{\Phi}\left(\mathbb{R}^{n}\right)
$$

To deal with the second sum in (3.32), by Hölder's inequality,

$$
\omega \in A_{q}\left(\mathbb{R}^{n}\right) \cap R H_{\frac{2}{2-q}}\left(\mathbb{R}^{n}\right),
$$

Lemma 2.2(v), (3.34), $\left|\widetilde{\chi}_{k+1}-\tilde{\chi}_{k}\right| \lesssim\left|2^{k} Q\right|^{-1}$, Lemma 2.2(iii) and the upper type $\frac{1}{p_{0}}-1$ property of $\rho$, we know that there exists a positive constant $C_{7}$ such that for all $k \in \mathbb{Z}_{+}$,

$$
\begin{aligned}
& \left\|N_{k+1}\left(\tilde{\chi}_{k+1}-\tilde{\chi}_{k}\right)\right\|_{L_{\omega}^{q}\left(\mathbb{R}^{n}\right)} \\
& \quad \leq\left\|N_{k+1}\left(\widetilde{\chi}_{k+1}-\tilde{\chi}_{k}\right)\right\|_{L^{2}\left(2^{k+1} Q\right)}\left\{\int_{2^{k+1} Q}[\omega(x)]^{\frac{2}{2-q}} d x\right\}^{\frac{1}{q}-\frac{1}{2}} \\
& \quad \leq\left|2^{k+1} Q\right|^{-\frac{1}{2}}\left|N_{k+1}\right| \frac{\left[\omega\left(2^{k+1} Q\right)\right]^{\frac{1}{q}}}{\left|2^{k+1} Q\right|^{\frac{1}{2}}} \\
& \quad \lesssim\left|2^{k+1} Q\right|^{-\frac{1}{2}}\left(\sum_{j=k+1}^{\infty} 2^{-j}\right)|Q|^{\frac{1}{2}}\|a\|_{T_{2}^{2}\left(\mathbb{R}^{n} \times\left(0, R_{0}\right]\right) \frac{\left[\omega\left(2^{k+1} Q\right)\right]^{\frac{1}{q}}}{\left|2^{k+1} Q\right|^{\frac{1}{2}}}} \\
& \quad \lesssim 2^{-k(n+1)} \frac{\left[\omega\left(2^{k+1} Q\right)\right]^{\frac{1}{q}}}{\omega(Q) \rho(\omega(Q))} \lesssim 2^{-k(n+1)} 2^{k n q} \frac{\left[\omega\left(2^{k+1} Q\right)\right]^{\frac{1}{q}}}{\omega\left(2^{k+1} Q\right) \rho\left(2^{-k n q} \omega\left(2^{k+1} Q\right)\right)} \\
& \lesssim C_{7} 2^{-k n\left(\frac{n+1}{n}-\frac{q}{p_{0}}\right)}\left[\omega\left(2^{k+1} Q\right)\right]^{\frac{1}{q}-1}\left[\rho\left(\omega\left(2^{k+1} Q\right)\right)\right]^{-1} .
\end{aligned}
$$

This, combined with

$$
\int_{\mathbb{R}^{n}}\left[\widetilde{\chi}_{k+1}(x)-\widetilde{\chi}_{k}(x)\right] d x=0
$$

and $\operatorname{supp}\left(\widetilde{\chi}_{k+1}-\widetilde{\chi}_{k}\right) \subset 2^{k+1} Q$, yields that for each $k \in \mathbb{Z}_{+}$,

$$
2^{-k n\left(\frac{n+1}{n}-\frac{q}{p_{0}}\right)} N_{k+1}\left(\widetilde{\chi}_{k+1}-\widetilde{\chi}_{k}\right) / C_{7}
$$

is a $(\rho, q, 0)_{\omega}$-atom. Moreover, by an argument similar to that used in [87, p. 44], we know that

$$
\sum_{k=0}^{\infty} N_{k+1}\left(\tilde{\chi}_{k+1}-\tilde{\chi}_{k}\right)
$$

converges in $L^{2}\left(\mathbb{R}^{n}\right)$. For all $k \in \mathbb{Z}_{+}$, let

$$
\lambda_{2, k}:=C_{7} 2^{-k n\left(\frac{n+1}{n}-\frac{q}{p_{0}}\right)} \text { and } a_{2, k}:=2^{k n\left(\frac{n+1}{n}-\frac{q}{p_{0}}\right)} N_{k+1}\left(\widetilde{\chi}_{k+1}-\widetilde{\chi}_{k}\right) / C_{7} .
$$

Then $\left\{a_{2, k}\right\}_{k=0}^{\infty}$ is a sequence of $(\rho, q, 0)_{\omega}$-atoms. Similar to the proof of (3.36), we also see that for all $\lambda \in(0, \infty)$,

$$
\sum_{k=0}^{\infty} \omega\left(2^{k+1} Q\right) \Phi\left(\frac{\left|\lambda_{2, k}\right|}{\lambda \omega\left(2^{k+1} Q\right) \rho\left(\omega\left(2^{k+1} Q\right)\right)}\right) \lesssim \omega(Q) \Phi\left(\frac{1}{\lambda \omega(Q) \rho(\omega(Q))}\right),
$$

which, together with Lemma 3.7, implies that

$$
\sum_{k=0}^{\infty} N_{k+1}\left(\widetilde{\chi}_{k+1}-\tilde{\chi}_{k}\right) \in h_{\omega}^{\Phi}\left(\mathbb{R}^{n}\right)
$$


Let $f \in L^{2}\left(\mathbb{R}^{n}\right)$ satisfy $\left\|S_{h, R_{0}}^{\text {loc }}(f)\right\|_{L_{\omega}^{\Phi}\left(\mathbb{R}^{n}\right)}<\infty$. It is easy to see that for all $z \in \mathbb{C}$ satisfying $z \neq 0$ and $|\arg z| \in(0, \pi / 2)$,

$$
8 \int_{0}^{R_{0}}\left(t^{2} z e^{-t^{2} z}\right)\left(t^{2} z e^{-t^{2} z}\right) \frac{d t}{t}+\left(2 R_{0}^{2} z+1\right) e^{-2 R_{0}^{2} z}=1
$$

which, together with the $H^{\infty}$-functional calculus for $L$ (see, for example, 62]), implies that

$$
\begin{aligned}
f & =8 \int_{0}^{R_{0}}\left(t^{2} L e^{-t^{2} L}\right)\left(t^{2} L e^{-t^{2} L}\right)(f) \frac{d t}{t}+\left[2 R_{0}^{2} L e^{-2 R_{0}^{2} L}(f)+e^{-2 R_{0}^{2} L}(f)\right] \\
& =: f_{1}+f_{2} .
\end{aligned}
$$

From the assumption $\left\|S_{h, R_{0}}^{\text {loc }}(f)\right\|_{L_{\omega}^{\Phi}\left(\mathbb{R}^{n}\right)}<\infty$, we deduce that

$$
t^{2} L e^{-t^{2} L}(f) \in T_{\Phi, \omega}^{\text {loc, } R_{0}}\left(\mathbb{R}^{n}\right)
$$

and

$$
\left\|S_{h, R_{0}}^{\text {loc }}(f)\right\|_{L_{\omega}^{\Phi}\left(\mathbb{R}^{n}\right)}=\left\|t^{2} L e^{-t^{2} L}(f)\right\|_{T_{\Phi, \omega}^{\text {loc, } R_{0}}\left(\mathbb{R}^{n}\right)} .
$$

Then by Lemma 3.14 we know that there exist $\left\{\mu_{j}\right\}_{j} \subset \mathbb{C}$ and a sequence $\left\{a_{j}\right\}_{j}$ of $T_{\Phi, \omega}^{\text {loc, } R_{0}}\left(\mathbb{R}^{n}\right)$-atoms such that for almost every $(x, t) \in \mathbb{R}^{n} \times\left(0, R_{0}\right]$,

$$
t^{2} L e^{-t^{2} L}(f)(x)=\sum_{j} \mu_{j} a_{j}(x, t) .
$$

For each $j$, let

$$
\alpha_{j}:=8 \int_{0}^{R_{0}} t^{2} L e^{-t^{2} L}\left(a_{j}\right) \frac{d t}{t} .
$$

Then by (3.39) and (3.40), similar to the proof of [49, Proposition 4.2], we see that

$$
f_{1}=\sum_{j} \mu_{j} \alpha_{j}
$$

in $L^{2}\left(\mathbb{R}^{n}\right)$. Replacing $\alpha$ in (3.28) by $\alpha_{j}$, consequently, we then denote $M_{k}, N_{k}$ and $\tilde{\chi}_{k}$ in (3.30), $\lambda_{1, k}, \lambda_{2, k}, a_{1, k}$ and $a_{2, k}$, respectively, by $M_{j, k}, N_{j, k}, \tilde{\chi}_{j, k}, \lambda_{1, j, k}$, $\lambda_{2, j, k}, a_{1, j, k}$ and $a_{2, j, k}$. Repeating the above procedure, we obtain

$$
\begin{aligned}
f_{1} & =\sum_{j} \sum_{k=0}^{\infty} \mu_{j} M_{j, k}+\sum_{j} \sum_{k=0}^{\infty} \mu_{j} N_{j, k+1}\left(\tilde{\chi}_{j, k+1}-\tilde{\chi}_{j, k}\right) \\
& =: \sum_{j} \sum_{k=0}^{\infty} \mu_{j} \lambda_{1, j, k} a_{1, j, k}+\sum_{j} \sum_{k=0}^{\infty} \mu_{j} \lambda_{2, j, k} a_{2, j, k},
\end{aligned}
$$

where for each $j$,

$$
\left\{\lambda_{1, j, k}\right\}_{k \in \mathbb{Z}_{+}} \cup\left\{\lambda_{1, j, k}\right\}_{k \in \mathbb{Z}_{+}} \subset \mathbb{C}
$$

and, $\left\{a_{1, j, k}\right\}_{k \in \mathbb{Z}_{+}}$and $\left\{a_{2, j, k}\right\}_{k \in \mathbb{Z}_{+}}$are sequences of $(\rho, q, 0)_{\omega}$-atoms and both summations hold in $L^{2}\left(\mathbb{R}^{n}\right)$, and hence in $\mathcal{D}^{\prime}\left(\mathbb{R}^{n}\right)$. Moreover, from (3.36) with $Q$ and $\lambda_{1, k}$ replaced by $Q_{j}$ and $\lambda_{1, j, k}$, (3.38) with $Q$ and $\lambda_{2, k}$ replaced by $Q_{j}$ and $\lambda_{2, j, k}$, and Lemma 3.14, we deduce that

$$
\begin{aligned}
\left.\Lambda\left(\left\{\mu_{j} \lambda_{1, j, k} a_{1, j, k}\right\}_{j, k}\right)+\Lambda\left(\left\{\mu_{j} \lambda_{1, j, k} a_{1, j, k}\right)\right\}_{j, k}\right) & \lesssim \Lambda\left(\left\{\mu_{j} a_{j}\right\}_{j}\right) \\
& \lesssim\left\|S_{h, R_{0}}^{\text {loc }}(f)\right\|_{L_{\omega}^{\Phi}\left(\mathbb{R}^{n}\right)} .
\end{aligned}
$$


This, combined with Lemma 3.7. implies that $f_{1} \in h_{\omega}^{\Phi}\left(\mathbb{R}^{n}\right)$ and

$$
\left\|f_{1}\right\|_{h_{\omega}^{\Phi}\left(\mathbb{R}^{n}\right)} \lesssim\left\|S_{h, R_{0}}^{\text {loc }}(f)\right\|_{L_{\omega}^{\Phi}\left(\mathbb{R}^{n}\right)} .
$$

Now we deal with $f_{2}$. Denote by $\widetilde{K}_{R_{0}}$ the kernel of $\left(2 R_{0}^{2} L+1\right) e^{-R_{0}^{2} L}$. Then by the mean value theorem for integrals, we know that for all $x \in \mathbb{R}^{n}$,

$$
\begin{aligned}
f_{2}(x) & =\int_{\mathbb{R}^{n}} \widetilde{K}_{R_{0}}(x, y) e^{-R_{0}^{2} L}(f)(y) d y=\sum_{Q_{k} \in \mathcal{Q}} \int_{Q_{k}} \widetilde{K}_{R_{0}}(x, y) e^{-R_{0}^{2} L}(f)(y) d y \\
& =\sum_{Q_{k} \in \mathcal{Q}}\left|Q_{k}\right| m_{Q_{k}}\left(e^{-R_{0}^{2} L}(f)\right) \widetilde{K}_{R_{0}}\left(x, y_{k}\right),
\end{aligned}
$$

where $\mathcal{Q}$ denotes the set of all unit cubes of $\mathbb{R}^{n}$ whose interiors are disjoint, and for each $k \in \mathbb{N}, y_{k} \in Q_{k}$ may depend on $x$. For each $k$, we have

$$
\widetilde{K}_{R_{0}}\left(x, y_{k}\right)=\sum_{i=0}^{\infty} \widetilde{K}_{R_{0}}\left(x, y_{k}\right) \chi_{S_{i}\left(Q_{k}\right)}=: \sum_{i=0}^{\infty} H_{k, i},
$$

where $S_{0}\left(Q_{k}\right):=2 Q_{k}$ and for each $i \in \mathbb{N}, S_{i}\left(Q_{k}\right):=2^{i+1} Q_{k} \backslash 2^{i} Q_{k}$. For each $k$, from (2.8), it follows that

$$
\left|\widetilde{K}_{R_{0}}\left(x, y_{k}\right)\right| \lesssim \frac{1}{\left(1+\left|x-y_{k}\right|\right)^{n+1}} .
$$

By this, we conclude that there exists a positive constant $C_{8}$ such that

$$
\left\|H_{k, 0}\right\|_{L_{\omega}^{q}\left(\mathbb{R}^{n}\right)} \lesssim\left\{C_{8} \omega\left(2 Q_{k}\right) \rho\left(\omega\left(2 Q_{k}\right)\right)\right\}\left[\omega\left(2 Q_{k}\right)\right]^{\frac{1}{q}-1}\left[\rho\left(\omega\left(2 Q_{k}\right)\right)\right]^{-1} .
$$

Thus, $\left\{C_{8} \omega\left(2 Q_{k}\right) \rho\left(\omega\left(2 Q_{k}\right)\right)\right\}^{-1} H_{k, 0}$ is a $(\rho, q, 0)_{\omega}$-atom. For all $i \in \mathbb{N}$, from (3.43), it follows that there exists a positive constant $C_{9}$ such that

$$
\begin{aligned}
\left\|H_{k, i}\right\|_{L_{\omega}^{q}\left(\mathbb{R}^{n}\right)} \lesssim & \left\{\int_{S_{i}\left(Q_{k}\right)} \frac{\omega(x)}{\left[2^{i} l\left(Q_{k}\right)\right]^{(n+1) q}} d x\right\}^{\frac{1}{q}} \\
\lesssim & \left\{C_{9}\left|2^{i} Q_{k}\right|^{-\frac{n+1}{n}} \omega\left(2^{i+1} Q_{k}\right) \rho\left(\omega\left(2^{i+1} Q_{k}\right)\right)\right\} \\
& \times\left[\omega\left(2^{i+1} Q_{k}\right)\right]^{\frac{1}{q}-1}\left[\rho\left(\omega\left(2^{i+1} Q_{k}\right)\right)\right]^{-1},
\end{aligned}
$$

which implies that $C_{9}^{-1}\left|2^{i} Q_{k}\right|^{\frac{n+1}{n}}\left[\omega\left(2^{i+1} Q_{k}\right) \rho\left(\omega\left(2^{i+1} Q_{k}\right)\right)\right]^{-1} H_{k, i}$ is a $(\rho, q, 0)_{\omega^{-}}$ atom. Let

$$
\lambda_{3, k, i}:=C_{9}\left|Q_{k}\right| m_{Q_{k}}\left(e^{-R_{0}^{2} L}(f)\right)\left|2^{i} Q_{k}\right|^{-\frac{n+1}{n}} \omega\left(2^{i+1} Q_{k}\right) \rho\left(\omega\left(2^{i+1} Q_{k}\right)\right)
$$

and

$$
a_{3, k, i}:=C_{9}^{-1}\left|2^{i} Q_{k}\right|^{\frac{n+1}{n}}\left[\omega\left(2^{i+1} Q_{k}\right) \rho\left(\omega\left(2^{i+1} Q_{k}\right)\right)\right]^{-1} H_{k, i}
$$

for $i \in \mathbb{N}$,

$$
\lambda_{3, k, 0}:=C_{8}\left|Q_{k}\right| m_{Q_{k}}\left(e^{-R_{0}^{2} L}(f)\right) \omega\left(2 Q_{k}\right) \rho\left(\omega\left(2 Q_{k}\right)\right)
$$

and

$$
a_{3, k, 0}:=\left\{C_{8} \omega\left(2 Q_{k}\right) \rho\left(\omega\left(2 Q_{k}\right)\right)\right\}^{-1} H_{k, 0} .
$$

Then

$$
f_{2}=\sum_{k} \sum_{i=0}^{\infty} \lambda_{3, k, i} a_{3, k, i}
$$


and $\left\{a_{3, k, i}\right\}_{k, i \in \mathbb{Z}_{+}}$is a sequence of $(\rho, q, 0)_{\omega}$-atoms. From this, (3.44), (3.45), $l\left(Q_{k}\right)=1$, the lower type $p_{0}$ property of $\Phi$ and the first inequality in (3.32), we deduce that for all $\lambda \in(0, \infty)$,

$$
\begin{aligned}
& \sum_{Q_{k} \in \mathcal{Q}} \sum_{i=0}^{\infty} \omega\left(2^{i+1} Q_{k}\right) \Phi\left(\frac{\left|\lambda_{3, k}\right|}{\lambda \omega\left(2^{i+1} Q_{k}\right) \rho\left(\omega\left(2^{i+1} Q_{k}\right)\right)}\right) \\
& \lesssim \sum_{Q_{k} \in \mathcal{Q}} \sum_{i=0}^{\infty} \omega\left(2^{i+1} Q_{k}\right) \Phi\left(\frac{2^{-i(n+1)} m_{Q_{k}}\left(\left|e^{-R_{0}^{2} L}(f)\right|\right)}{\lambda}\right) \\
& \lesssim \sum_{Q_{k} \in \mathcal{Q}} \sum_{i=0}^{\infty} 2^{-i n q} 2^{-(n+1) p_{0}} \omega\left(Q_{k}\right) \Phi\left(\frac{m_{Q_{k}}\left(\left|e^{-R_{0}^{2} L}(f)\right|\right)}{\lambda}\right) \\
& \lesssim \sum_{Q_{k} \in \mathcal{Q}} \omega\left(Q_{k}\right) \Phi\left(\frac{m_{Q_{k}}\left(\left|e^{-R_{0}^{2} L}(f)\right|\right)}{\lambda}\right),
\end{aligned}
$$

which, together with Lemma 3.7 implies that $f_{2} \in h_{\omega}^{\Phi}\left(\mathbb{R}^{n}\right)$ and

$$
\left\|f_{2}\right\|_{h_{\omega}^{\Phi}\left(\mathbb{R}^{n}\right)} \lesssim \inf \left\{\lambda \in(0, \infty): \sum_{Q_{k} \in \mathcal{Q}} \omega\left(Q_{k}\right) \Phi\left(\frac{m_{Q_{k}}\left(\left|e^{-R_{0}^{2} L}(f)\right|\right)}{\lambda}\right) \leq 1\right\} .
$$

From this, (3.42) and (3.46), we infer that $f \in h_{\omega}^{\Phi}\left(\mathbb{R}^{n}\right)$ and

$$
\begin{aligned}
\|f\|_{h \omega}\left(\mathbb{R}^{n}\right) \lesssim & \left\|S_{h, R_{0}}^{\text {loc }}(f)\right\|_{L_{\omega}^{\Phi}\left(\mathbb{R}^{n}\right)} \\
& +\inf \left\{\lambda \in(0, \infty): \sum_{Q_{k} \in \mathcal{Q}} \omega\left(Q_{k}\right) \Phi\left(\frac{m_{Q_{k}}\left(\left|e^{-R_{0}^{2} L}(f)\right|\right)}{\lambda}\right) \leq 1\right\},
\end{aligned}
$$

which completes the proof of Proposition 3.13(i).

Now we prove Proposition 3.13(ii). Let $f \in L^{2}(\Omega)$ satisfy $\left\|S_{h, R_{0}}^{\text {loc }}(f)\right\|_{L_{\omega}^{\Phi}(\Omega)}<\infty$. Similar to the proof of (3.39), we know that (3.39) also holds in this case. Let $f_{1}$ and $f_{2}$ be as in (3.39).

We first deal with $f_{1}$. Similar to the proof of (3.41), we know that

$$
f_{1}=\sum_{j} \mu_{j} \alpha_{j}
$$

in $L^{2}(\Omega)$, where $\left\{\mu_{j}\right\}_{j} \subset \mathbb{C}$ and for each $j$,

$$
\alpha_{j}:=8 \int_{0}^{R_{0}} t^{2} L e^{-t^{2} L}\left(a_{j}\right) \frac{d t}{t}
$$

and $a_{j}$ is a $T_{\Phi, \omega}^{\text {loc, } R_{0}}(\Omega)$-atom. For any $T_{\Phi, \omega}^{\text {loc, } R_{0}}(\Omega)$-atom $a$ supported in $\widehat{Q \cap \Omega}$, let

$$
\alpha:=8 \int_{0}^{R_{0}} t^{2} L e^{-t^{2} L}(a) \frac{d t}{t} .
$$

To show $f_{1} \in h_{\omega, r}^{\Phi}(\Omega)$, it suffices to show that there exist a function $\widetilde{\alpha}$ on $\mathbb{R}^{n}$, a sequence $\left\{\lambda_{i}\right\}_{i}$ of numbers and a sequence $\left\{b_{i}\right\}_{i}$ of $(\rho, q, 0)_{\omega}$-atoms such that

$$
\begin{gathered}
\left.\widetilde{\alpha}\right|_{\Omega}=\alpha, \\
\widetilde{\alpha}=\sum_{i} \lambda_{i} b_{i} \text { in } L^{2}\left(\mathbb{R}^{n}\right)
\end{gathered}
$$


and for all $\lambda \in(0, \infty)$,

$$
\sum_{i} \omega\left(Q_{i}\right) \Phi\left(\frac{\left|\lambda_{i}\right|}{\lambda \omega\left(Q_{i}\right) \rho\left(\omega\left(Q_{i}\right)\right)}\right) \lesssim \omega(Q \cap \Omega) \Phi\left(\frac{1}{\lambda \omega(Q \cap \Omega) \rho(\omega(Q \cap \Omega))}\right),
$$

where for each $i, \operatorname{supp}\left(b_{i}\right) \subset Q_{i}$ and $Q \cap \Omega$ appears in the support of $a$. Indeed, if (3.47) and (3.48) hold, then by (3.47), we know that for each $j$, there exists a function $\widetilde{\alpha}_{j}$ on $\mathbb{R}^{n}$ such that $\left.\widetilde{\alpha}_{j}\right|_{\Omega}=\alpha_{j}$. Let

$$
\widetilde{f}_{1}:=\sum_{j} \mu_{j} \widetilde{\alpha}_{j}
$$

Then $\left.\widetilde{f}_{1}\right|_{\Omega}=f_{1}$. Furthermore, from (3.48), we deduce that there exist $\left\{\lambda_{j, i}\right\}_{j, i} \subset \mathbb{C}$ and a sequence $\left\{b_{j, i}\right\}_{j, i}$ of $(\rho, q, 0)_{\omega}$-atoms such that

$$
\tilde{f}_{1}=\sum_{j} \sum_{i} \mu_{j} \lambda_{j, i} b_{j, i}
$$

and for all $\lambda \in(0, \infty)$,

$$
\begin{aligned}
& \sum_{j, i} \omega\left(Q_{j, i}\right) \Phi\left(\frac{\left|\mu_{j} \lambda_{j, i}\right|}{\lambda \omega\left(Q_{j, i}\right) \rho\left(\omega\left(Q_{j, i}\right)\right)}\right) \\
& \quad \lesssim \sum_{j} \omega\left(Q_{j} \cap \Omega\right) \Phi\left(\frac{\left|\mu_{j}\right|}{\lambda \omega\left(Q_{j} \cap \Omega\right) \rho\left(\omega\left(Q_{j} \cap \Omega\right)\right)}\right),
\end{aligned}
$$

which, together with Lemmas 3.7 and 3.14, implies that

$$
\left\|\widetilde{f}_{1}\right\|_{h_{\omega}^{\Phi}\left(\mathbb{R}^{n}\right)} \sim\left\|\widetilde{f}_{1}\right\|_{h_{\omega}^{\rho, q, 0}\left(\mathbb{R}^{n}\right)} \lesssim\left\|S_{h, R_{0}}^{\text {loc }}(f)\right\|_{L_{\omega}^{\Phi}(\Omega)} .
$$

From this and Definition 1.3, we deduce that $\widetilde{f}_{1} \in h_{\omega, r}^{\Phi}(\Omega)$ and

$$
\left\|f_{1}\right\|_{h_{\omega, r}^{\Phi}(\Omega)} \lesssim\left\|S_{h, R_{0}}^{\text {loc }}(f)\right\|_{L_{\omega}^{\Phi}(\Omega)} .
$$

Let $Q:=Q\left(x_{0}, r_{0}\right)$. Now we show (3.47) and (3.48) by considering the following two cases for $Q$ which appears in the support of $a$.

Case 1) $8 Q \cap \Omega^{\complement} \neq \emptyset$. In this case, let

$$
R_{k}(Q):=\left(2^{k+1} Q \backslash 2^{k} Q\right) \cap \Omega
$$

if $k \geq 3$ and $R_{0}(Q):=8 Q \cap \Omega$. Let

$$
J_{\Omega}:=\left\{k \in \mathbb{N}: k \geq 3,\left|R_{k}(Q)\right|>0\right\} .
$$

For $k \in J_{\Omega} \cup\{0\}$, let $\chi_{k}:=\chi_{R_{k}(Q)}, \widetilde{\chi}_{k}:=\left|R_{k}(Q)\right|^{-1} \chi_{k}$ and

$$
m_{k}:=\int_{R_{k}(Q)} \alpha(x) d x .
$$

Then we have

$$
\alpha=\alpha \chi_{0}+\sum_{k \in J_{\Omega}} \alpha \chi_{k}
$$

almost everywhere and also in $L^{2}(\Omega)$. Take the cube $\widetilde{Q} \subset \mathbb{R}^{n}$ such that the center of $\widetilde{Q}, x_{\widetilde{Q}} \in \Omega^{\complement}, l(\widetilde{Q})=l(Q)$ and $\operatorname{dist}(Q, \widetilde{Q}) \sim l(Q)$. Then there exists a cube $Q_{0}^{*}$ such that $(8 Q \cup \widetilde{Q}) \subset Q_{0}^{*}$ and

$$
l\left(Q_{0}^{*}\right) \sim l(Q) .
$$


Let

$$
H_{0}:=\alpha \chi_{0}-\frac{1}{\left|\widetilde{Q} \cap \Omega^{\complement}\right|}\left\{\int_{R_{0}(Q)} \alpha(x) d x\right\} \chi_{\widetilde{Q} \cap \Omega^{\mathrm{C}}} .
$$

Then

$$
\int_{\mathbb{R}^{n}} H_{0}(x) d x=0
$$

and $\operatorname{supp}\left(H_{0}\right) \subset Q_{0}^{*}$. Similar to the proof of [87, (3.51)], we conclude that

$$
\|\alpha\|_{L^{2}(\Omega)} \lesssim\|a\|_{T_{2}^{2}\left(\Omega \times\left(0, R_{0}\right]\right)}
$$

By the assumption that $\Omega^{\complement}$ is an unbounded strongly Lipschitz domain and Lemma 3.10, we know that $\left|\widetilde{Q} \cap \Omega^{\complement}\right| \sim|\widetilde{Q}|$. From this, Hölder's inequality,

$$
\omega \in A_{q}\left(\mathbb{R}^{n}\right) \cap R H_{2 /(2-q)}\left(\mathbb{R}^{n}\right),
$$

(2.3), (3.50), (3.51) and, (iii) and (v) of Lemma 2.2, it follows that there exists a positive constant $C_{10}$ such that

$$
\begin{aligned}
\left\|H_{0}\right\|_{L_{\omega}^{q}\left(\mathbb{R}^{n}\right)} & \leq\left\|H_{0}\right\|_{L^{2}\left(Q_{0}^{*}\right)}\left\{\int_{Q_{0}^{*}}[\omega(x)]^{2 /(2-q)} d x\right\}^{\frac{1}{q}-\frac{1}{2}} \\
& \lesssim\left\|H_{0}\right\|_{L^{2}\left(Q_{0}^{*}\right)} \frac{\left[\omega\left(Q_{0}^{*}\right)\right]^{\frac{1}{q}}}{\left|Q_{0}^{*}\right|^{\frac{1}{2}}} \\
& \lesssim\left\{\|\alpha\|_{L^{2}(\Omega)}+\frac{1}{\left|\widetilde{Q} \cap \Omega^{\complement}\right|^{\frac{1}{2}}}\left(\int_{R_{0}(Q)}|\alpha(x)|^{2} d x\right)^{\frac{1}{2}}|Q \cap \Omega|^{\frac{1}{2}}\right\} \frac{\left[\omega\left(Q_{0}^{*}\right)\right]^{\frac{1}{q}}}{\left|Q_{0}^{*}\right|^{\frac{1}{2}}} \\
& \lesssim \frac{\left[\omega\left(Q_{0}^{*}\right)\right]^{\frac{1}{q}}}{\left|Q_{0}^{*}\right|^{\frac{1}{2}}}\|\alpha\|_{L^{2}(\Omega)} \lesssim \frac{\left[\omega\left(Q_{0}^{*}\right)\right]^{\frac{1}{q}}}{\left|Q_{0}^{*}\right|^{\frac{1}{2}}}\|a\|_{T_{2}^{2}\left(\Omega \times\left(0, R_{0}\right]\right)} \\
& \lesssim \frac{\left[\omega\left(Q_{0}^{*}\right)\right]^{\frac{1}{q}}|Q \cap \Omega|^{\frac{1}{2}}}{\left|Q_{0}^{*}\right|^{\frac{1}{2}} \omega(Q \cap \Omega) \rho(\omega(Q \cap \Omega))} \lesssim C_{10}\left[\omega\left(Q_{0}^{*}\right)\right]^{\frac{1}{q}-1}\left[\rho\left(\omega\left(Q_{0}^{*}\right)\right)\right]^{-1} .
\end{aligned}
$$

Let $\lambda_{0}:=C_{10}$ and $b_{0}:=H_{0} / C_{10}$. Then $H_{0}=\lambda_{0} b_{0}$ and $b_{0}$ is a $(\rho, q, 0)_{\omega}$-atom.

To finish the proof in this case, we need the following Fact 1, whose proof is similar to the usual Whitney decomposition of an open set in $\mathbb{R}^{n}$; see, for example, [76]. We omit the details.

Fact 1 . For all $k \in J_{\Omega}$, there exists the Whitney decomposition $\left\{Q_{k, i}\right\}_{i}$ of $R_{k}(Q)$ about $\partial \Omega$, where $\left\{Q_{k}, i\right\}_{i}$ are dyadic cubes of $\mathbb{R}^{n}$ with disjoint interiors and for each $i, 2 Q_{k, i} \subset \Omega$ but $4 Q_{k, i} \cap \partial \Omega \neq \emptyset$.

Let $\left\{Q_{k, i}\right\}_{k \in J_{\Omega}, i}$ be as in Fact 1. Then for each $k \in J_{\Omega}$,

$$
\alpha \chi_{R_{k}(Q)}=\sum_{i} \alpha \chi_{Q_{k, i}}
$$

almost everywhere. Similar to the proof of [87, (3.53)], we know that for all $x \in$ $R_{k}(Q)$

$$
|\alpha(x)| \lesssim 2^{-k(n+1)}|Q \cap \Omega|^{-\frac{1}{2}}\|a\|_{T_{2}^{2}\left(\Omega \times\left(0, R_{0}\right]\right)}
$$


Moreover, by Lemma 3.15 we see that for each $k$ and $i$, there exists a cube $\widetilde{Q}_{k, i} \subset$ $\Omega^{\complement}$ such that $l\left(\widetilde{Q}_{k, i}\right)=l\left(Q_{k, i}\right)$ and

$$
\operatorname{dist}\left(\widetilde{Q}_{k, i}, Q_{k, i}\right) \sim l\left(Q_{k, i}\right) .
$$

Then for each $k$ and $i$, there exists a cube $Q_{k, i}^{*}$ such that $\left(Q_{k, i} \cup \widetilde{Q}_{k, i}\right) \subset Q_{k, i}^{*}$ and $l\left(Q_{k, i}^{*}\right) \sim l\left(Q_{k, i}\right)$. For each $k$ and $i$, let

$$
H_{k, i}:=\alpha \chi_{Q_{k, i}}-\frac{1}{\left|\widetilde{Q}_{k, i}\right|}\left\{\int_{Q_{k, i}} \alpha(x) d x\right\} \chi_{\widetilde{Q}_{k, i}} .
$$

Then

$$
\int_{\mathbb{R}^{n}} H_{k, i}(x) d x=0 \quad \text { and } \quad \operatorname{supp}\left(H_{k, i}\right) \subset Q_{k, i}^{*} .
$$

Furthermore, from Hölder's inequality, $\omega \in R H_{2 /(2-q)}\left(\mathbb{R}^{n}\right)$, (2.3), (3.52) and

$$
l\left(Q_{k, i}^{*}\right) \sim l\left(Q_{k, i}\right),
$$

we infer that there exists a positive constant $C_{11}$ such that

$$
\begin{aligned}
\left\|H_{k, i}\right\|_{L_{\omega}^{q}\left(\mathbb{R}^{n}\right)} & \leq\|\alpha\|_{L^{2}\left(Q_{k, i}^{*}\right)}\left\{\int_{Q_{k, i}^{*}}[\omega(x)]^{2 /(2-q)} d x\right\}^{\frac{1}{q}-\frac{1}{2}} \\
& \lesssim 2^{-k(n+1)}|Q \cap \Omega|^{-\frac{1}{2}}\left|Q_{k, i}^{*}\right|^{\frac{1}{2}}\|a\|_{T_{2}^{2}\left(\Omega \times\left(0, R_{0}\right]\right)} \frac{\left[\omega\left(Q_{k, i}^{*}\right)\right]^{\frac{1}{q}}}{\left|Q_{k, i}^{*}\right|^{\frac{1}{2}}} \\
& \lesssim C_{11} 2^{-k(n+1)}\left[\omega\left(Q_{k, i}^{*}\right)\right]^{\frac{1}{q}}[\omega(Q \cap \Omega) \rho(\omega(Q \cap \Omega))]^{-1}
\end{aligned}
$$

For each $k$ and $j$, let

$$
\lambda_{k, j}:=\frac{C_{11} 2^{-k(n+1)} \omega\left(Q_{k, i}^{*}\right) \rho\left(\omega\left(Q_{k, i}^{*}\right)\right)}{\omega(Q \cap \Omega) \rho(\omega(Q \cap \Omega))}
$$

and

$$
b_{k, j}:=\frac{2^{k(n+1)} H_{k, j} \omega(Q \cap \Omega) \rho(\omega(Q \cap \Omega))}{C_{11} \omega\left(Q_{k, i}^{*}\right) \rho\left(\omega\left(Q_{k, i}^{*}\right)\right)} .
$$

Then for each $k$ and $j, b_{k, j}$ is a $(\rho, q, 0)_{\omega}$-atom and $H_{k, j}:=\lambda_{k, j} b_{k, j}$. Let

$$
\widetilde{\alpha}:=H_{0}+\sum_{k \in J_{\Omega}} \sum_{i} H_{k, i}=\lambda_{0} b_{0}+\sum_{k \in J_{\Omega}} \sum_{i} \lambda_{k, i} b_{k, j} .
$$

Then by the constructions of $H_{0}$ and $\left\{H_{k, i}\right\}_{k \in J_{\Omega}, i}$, we know that $\left.\widetilde{\alpha}\right|_{\Omega}=\alpha$. Similar to [87. (3.55)], we know that

$$
\widetilde{\alpha}=\lambda_{0} b_{0}+\sum_{k \in J_{\Omega}} \sum_{i} \lambda_{k, i} b_{k, j}
$$

in $L^{2}\left(\mathbb{R}^{n}\right)$. Moreover, by the definitions of $\lambda_{0}$ and $\lambda_{k, i}$, the lower type $p_{0}$ property of $\Phi$, Lemma 2.2 (iii) and the first inequality in (3.32), we know that for all $\lambda \in(0, \infty)$,

$$
\begin{aligned}
& \omega\left(Q_{0}^{*}\right) \Phi\left(\frac{\lambda_{0}}{\lambda \omega\left(Q_{0}^{*}\right) \rho\left(\omega\left(Q_{0}^{*}\right)\right)}\right)+\sum_{k \in J_{\Omega}} \sum_{i} \omega\left(Q_{k, i}^{*}\right) \Phi\left(\frac{\lambda_{k, i}}{\lambda \omega\left(Q_{k, i}^{*}\right) \rho\left(\omega\left(Q_{k, i}^{*}\right)\right)}\right) \\
& \quad \lesssim \omega(Q \cap \Omega) \Phi\left(\frac{1}{\lambda \omega(Q \cap \Omega) \rho(\omega(Q \cap \Omega))}\right)
\end{aligned}
$$




$$
\begin{aligned}
& +\sum_{k \in J_{\Omega}} \sum_{i} \omega\left(Q_{k, i}\right) \Phi\left(\frac{2^{-k(n+1)}}{\lambda \omega(Q \cap \Omega) \rho(\omega(Q \cap \Omega))}\right) \\
\lesssim & \omega(Q \cap \Omega) \Phi\left(\frac{1}{\lambda \omega(Q \cap \Omega) \rho(\omega(Q \cap \Omega))}\right) \\
& +\sum_{k=3}^{\infty} \omega\left(2^{k+1} Q \cap \Omega\right) \Phi\left(\frac{2^{-k(n+1)}}{\lambda \omega(Q \cap \Omega) \rho(\omega(Q \cap \Omega))}\right) \\
\lesssim & \omega(Q \cap \Omega) \Phi\left(\frac{1}{\lambda \omega(Q \cap \Omega) \rho(\omega(Q \cap \Omega))}\right)\left\{1+\sum_{k=3}^{\infty} 2^{-\left[k(n+1) p_{0}-k q n\right]}\right\} \\
\lesssim & \omega(Q \cap \Omega) \Phi\left(\frac{1}{\omega(Q \cap \Omega) \rho(\omega(Q \cap \Omega))}\right),
\end{aligned}
$$

which implies that (3.48) holds in Case 1).

Case 2) $8 Q \subset \Omega$. In this case, let $k_{0} \in \mathbb{N}$ be such that $2^{k_{0}} Q \subset \Omega$ but

$$
2^{k_{0}+1} Q \cap \partial \Omega \neq \emptyset \text {. }
$$

Then $k_{0} \geq 3$. Let

$$
R_{k}(Q):=\left(2^{k+1} Q \backslash 2^{k} Q\right) \cap \Omega
$$

for $k \in \mathbb{N}$, and $R_{0}(Q):=2 Q$. Let

$$
J_{\Omega, k_{0}}:=\left\{k \in \mathbb{N}: k \geq k_{0}+1,\left|R_{k}(Q)\right|>0\right\} .
$$

For $k \in \mathbb{Z}_{+}$, let $\chi_{k}:=\chi_{R_{k}(Q)}, \tilde{\chi}_{k}:=\left|R_{k}(Q)\right|^{-1} \chi_{k}$,

$$
m_{k}:=\int_{R_{k}(Q)} \alpha(x) d x, \quad M_{k}:=\alpha \chi_{k}-m_{k} \tilde{\chi}_{k}
$$

and $\widetilde{M}_{k}:=\alpha \chi_{k}$. Then

$$
\alpha=\sum_{k=0}^{k_{0}} M_{k}+\sum_{k \in J_{\Omega}, k_{0}} \widetilde{M}_{k}+\sum_{k=0}^{k_{0}} m_{k} \widetilde{\chi}_{k} .
$$

For $k \in\left\{0,1, \cdots, k_{0}\right\}$, by the definition of $M_{k}$, we know that

$$
\int_{\mathbb{R}^{n}} M_{k}(x) d x=0
$$

and $\operatorname{supp}\left(M_{k}\right) \subset 2^{k+1} Q$. Moreover, similar to the estimates of (3.33) and (3.35), we see that there exists a positive constant $C_{12}$ such that for all $k \in\left\{0,1, \cdots, k_{0}\right\}$,

$$
\left\|M_{k}\right\|_{L_{\omega}^{q}\left(\mathbb{R}^{n}\right)} \leq C_{12} 2^{-k n\left(\frac{n+1}{n}-\frac{q}{p_{0}}\right)}\left[\omega\left(2^{k+1} Q\right)\right]^{\frac{1}{q}-1}\left[\rho\left(\omega\left(2^{k+1} Q\right)\right)\right]^{-1} .
$$

For each $k \in\left\{0, \cdots, k_{0}\right\}$, let

$$
\lambda_{1, k}:=C_{12} 2^{-k n\left(\frac{n+1}{n}-\frac{q}{p_{0}}\right)} \text { and } b_{1, k}:=C_{12}^{-1} 2^{k n\left(\frac{n+1}{n}-\frac{q}{p_{0}}\right)} M_{k} .
$$

Thus, for each $k \in\left\{0, \cdots, k_{0}\right\}, b_{1, k}$ is a $(\rho, q, 0)_{\omega}$-atom and $M_{k}=\lambda_{1, k} b_{1, k}$. Moreover, similar to the estimate of (3.36), we know that for all $\lambda \in(0, \infty)$,

$$
\begin{aligned}
& \sum_{k=0}^{k_{0}} \omega\left(2^{k+1} Q\right) \Phi\left(\frac{\lambda_{1, k}}{\lambda \omega\left(2^{k+1} Q\right) \rho\left(\omega\left(2^{k+1} Q\right)\right)}\right) \\
& \quad \lesssim \omega(Q) \Phi\left(\frac{1}{\lambda \omega(Q) \rho(\omega(Q))}\right) .
\end{aligned}
$$


For each $k \in J_{\Omega, k_{0}}$, by Fact 1 , there exists the Whitney decomposition $\left\{Q_{k, i}\right\}_{i}$ of $R_{k}(Q)$ about $\partial \Omega$ such that $\bigcup_{i} Q_{k, i}=R_{k}(Q)$ and for each $i, Q_{i}$ satisfies $2 Q_{k, i} \subset \Omega$ and $4 Q_{k, i} \cap \partial \Omega \neq \emptyset$. Then

$$
\widetilde{M}_{k}=\sum_{i} \alpha \chi_{Q_{k, i}}
$$

almost everywhere. Moreover, by Lemma 3.15, for each $k$ and $i$, there exists a cube $\widetilde{Q}_{k, i} \subset \Omega^{\complement}$ such that $l\left(\widetilde{Q}_{k, i}\right)=l\left(Q_{k, i}\right)$ and $\operatorname{dist}\left(\widetilde{Q}_{k, i}, Q_{k, i}\right) \sim l\left(Q_{k, i}\right)$. Then for each $k$ and $i$, there exists a cube $Q_{k, i}^{*}$ such that $Q_{k, i} \cup \widetilde{Q}_{k, i} \subset Q_{k, i}^{*}$ and $l\left(Q_{k, i}^{*}\right) \sim$ $l\left(Q_{k, i}\right)$. For each $k$ and $i$, let

$$
H_{k, i}:=\alpha \chi_{Q_{k, i}}-\frac{1}{\left|\widetilde{Q}_{k, i}\right|}\left\{\int_{Q_{k, i}} \alpha(x) d x\right\} \chi_{\widetilde{Q}_{k, i}} .
$$

Then

$$
\int_{\mathbb{R}^{n}} H_{k, i}(x) d x=0
$$

and $\operatorname{supp}\left(H_{k, i}\right) \subset Q_{k, i}^{*}$. Furthermore, similar to the proof of (3.53), we conclude that there exists a positive constant $C_{13}$ such that for each $k \in J_{\Omega, k_{0}}$ and $i$,

$$
\left\|H_{k, i}\right\|_{L_{\omega}^{q}\left(\mathbb{R}^{n}\right)} \leq C_{13} 2^{-k(n+1)}\left[\omega\left(Q_{k, i}^{*}\right)\right]^{\frac{1}{q}}[\omega(Q \cap \Omega) \rho(\omega(Q \cap \Omega))]^{-1} .
$$

For each $k$ and $j$, let

$$
\lambda_{k, j}:=\frac{C_{13} 2^{-k(n+1)} \omega\left(Q_{k, i}^{*}\right) \rho\left(\omega\left(Q_{k, i}^{*}\right)\right)}{\omega(Q \cap \Omega) \rho(\omega(Q \cap \Omega))}
$$

and

$$
b_{k, j}:=\frac{2^{k(n+1)} H_{k, j} \omega(Q \cap \Omega) \rho(\omega(Q \cap \Omega))}{C_{13} \omega\left(Q_{k, i}^{*}\right) \rho\left(\omega\left(Q_{k, i}^{*}\right)\right)} .
$$

Then for each $k$ and $j, b_{k, j}$ is a $(\rho, q, 0)_{\omega}$-atom and $H_{k, j}:=\lambda_{k, j} b_{k, j}$. Furthermore, similar to the proof of (3.54), we see that for all $\lambda \in(0, \infty)$,

$$
\begin{gathered}
\sum_{k \in J_{\Omega, k_{0}}} \sum_{i} \omega\left(Q_{k, i}^{*}\right) \Phi\left(\frac{\lambda_{k, i}}{\lambda \omega\left(Q_{k, i}^{*}\right) \rho\left(\omega\left(Q_{k, i}^{*}\right)\right)}\right) \\
\lesssim \omega(Q \cap \Omega) \Phi\left(\frac{1}{\lambda \omega(Q \cap \Omega) \rho(\omega(Q \cap \Omega))}\right) .
\end{gathered}
$$

For $j \in\left\{0,1, \cdots, k_{0}\right\}$, let

$$
N_{j}:=\sum_{k=j}^{k_{0}} m_{k}
$$

It is easy to see that

$$
\sum_{k=0}^{k_{0}} m_{k} \tilde{\chi}_{k}=\sum_{k=1}^{k_{0}}\left(\widetilde{\chi}_{k}-\tilde{\chi}_{k-1}\right) N_{k}+N_{0} \tilde{\chi}_{0} .
$$

Similar to the proof of (3.37), we know that there exists a positive constant $C_{14}$ such that for each $k \in\left\{1,2, \cdots, k_{0}\right\}$,

$$
\begin{aligned}
& \left\|N_{k}\left(\tilde{\chi}_{k}-\widetilde{\chi}_{k-1}\right)\right\|_{L_{\omega}^{q}\left(\mathbb{R}^{n}\right)} \\
& \quad \leq C_{14} 2^{-k n\left(\frac{n+1}{n}-\frac{q}{p_{0}}\right)}\left[\omega\left(2^{k} Q\right)\right]^{\frac{1}{q}-1}\left[\rho\left(\omega\left(2^{k} Q\right)\right)\right]^{-1} .
\end{aligned}
$$


For all $k \in\left\{1,2, \cdots, k_{0}\right\}$, let

$$
\lambda_{2, k}:=C_{14} 2^{-k n\left(\frac{n+1}{n}-\frac{q}{p_{0}}\right)} \text { and } b_{2, k}:=C_{14}^{-1} 2^{k n\left(\frac{n+1}{n}-\frac{q}{p_{0}}\right)} N_{k}\left(\widetilde{\chi}_{k}-\widetilde{\chi}_{k-1}\right) .
$$

Then from (3.57),

$$
\int_{\mathbb{R}^{n}}\left[\widetilde{\chi}_{k}(x)-\widetilde{\chi}_{k-1}(x)\right] d x=0
$$

and $\operatorname{supp}\left(\widetilde{\chi}_{k}-\widetilde{\chi}_{k-1}\right) \subset 2^{k} Q$, we deduce that for each $k \in\left\{1,2, \cdots, k_{0}\right\}, b_{2, k}$ is a $(\rho, q, 0)_{\omega}$-atom. Similar to the proof of (3.36), we know that for all $\lambda \in(0, \infty)$,

$$
\sum_{k=1}^{k_{0}} \omega\left(2^{k} Q\right) \Phi\left(\frac{\lambda_{2, k}}{\lambda \omega\left(2^{k} Q\right) \rho\left(\omega\left(2^{k} Q\right)\right)}\right) \lesssim \omega(Q) \Phi\left(\frac{1}{\lambda \omega(Q) \rho(\omega(Q))}\right) .
$$

Finally we deal with $N_{0} \widetilde{\chi}_{0}$. By

$$
2^{k_{0}-1} r_{0}<\operatorname{dist}\left(x_{0}, \partial \Omega\right) \leq 2^{k_{0}} r_{0},
$$

we conclude that there exist a positive integer $M$ and a sequence $\left\{Q_{0, i}\right\}_{i=1}^{M}$ of cubes such that

(i) $M \sim 2^{k_{0}}$;

(ii) for all $i \in\{1,2, \cdots, M\}, l\left(Q_{0, i}\right)=2 r_{0}$ and $Q_{0, i} \subset \Omega$;

(iii) for all $i \in\{1,2, \cdots, M-1\}, Q_{0, i} \cap Q_{0, i+1} \neq \emptyset$ and

$$
\operatorname{dist}\left(Q_{0, i}, \partial \Omega\right) \geq \operatorname{dist}\left(Q_{0, i+1}, \partial \Omega\right) \text {; }
$$

(iv) $2 Q_{0,} \cap \partial \Omega \neq \emptyset$.

Then by Lemma 3.15, there exists a cube $Q_{0, M+1} \subset \Omega^{\complement}$ such that $l\left(Q_{0, M+1}\right)=r_{0}$ and

$$
\operatorname{dist}\left(Q_{0, M}, Q_{0, M+1}\right) \sim r_{0} \text {. }
$$

Let

and

$$
H_{0,1}:=N_{0} \widetilde{\chi}_{0}-\frac{N_{0}}{\left|Q_{0,1}\right|} \chi_{Q_{0,1}}
$$

$$
H_{0, i}:=\frac{N_{0}}{\left|Q_{0, i-1}\right|} \chi_{Q_{0, i-1}}-\frac{N_{0}}{\left|Q_{0, i}\right|} \chi_{Q_{0, i}}
$$

with $i \in\{2, \cdots, M+1\}$. Obviously, for all $i \in\{1,2, \cdots, M+1\}$, by the definition of $H_{0, i}$, we see that

$$
\int_{\mathbb{R}^{n}} H_{0, i}(x) d x=0
$$

and there exists a cube $Q_{0, i}^{*} \subset \mathbb{R}^{n}$ such that $\operatorname{supp}\left(H_{0, i}\right) \subset Q_{0, i}^{*}$ and

$$
l\left(Q_{0, i}^{*}\right) \sim l(Q) .
$$

Similar to the proof of [87, (3.66)], we know that

$$
\left|N_{0}\right| \lesssim 2^{-k_{0}(n+1) / n}|Q|^{\frac{1}{2}}\|a\|_{T_{2}^{2}\left(\Omega \times\left(0, R_{0}\right]\right)} \cdot
$$

For each $i \in\{1,2, \cdots, M+1\}$, from Hölder's inequality, $\omega \in R H_{\frac{2}{2-q}}\left(\mathbb{R}^{n}\right)$, (2.3), the definition of $H_{0, i},(3.59)$ and (3.60), it follows that there exists a positive constant $C_{15}$ such that

$$
\left\|H_{0, i}\right\|_{L_{\omega}^{q}\left(\mathbb{R}^{n}\right)} \leq\left\|H_{0, i}\right\|_{L^{2}\left(\mathbb{R}^{n}\right)}\left\{\int_{Q_{0, i}^{*}}[\omega(x)]^{\frac{2}{2-q}} d x\right\}^{\frac{1}{q}-\frac{1}{2}}
$$




$$
\begin{aligned}
& \lesssim\left|N_{0}\right||Q|^{-\frac{1}{2}} \frac{\left[\omega\left(Q_{0, i}^{*}\right)\right]^{\frac{1}{q}}}{\left|Q_{0, i}^{*}\right|^{\frac{1}{2}}} \\
& \lesssim 2^{-k_{0}(n+1) / n} \frac{\left[\omega\left(Q_{0, i}^{*}\right)\right]^{\frac{1}{q}}}{\left|Q_{0, i}^{*}\right|^{\frac{1}{2}}}\|a\|_{T_{2}^{2}\left(\Omega \times\left(0, R_{0}\right]\right)} \\
& \lesssim C_{15} 2^{-k_{0}(n+1) / n}\left[\omega\left(Q_{0, i}^{*}\right)\right]^{\frac{1}{q}}[\omega(Q) \rho(\omega(Q))]^{-1} .
\end{aligned}
$$

For each $i \in\{1, \cdots, M+1\}$, let

$$
\lambda_{3, i}:=C_{15} 2^{-k_{0}(n+1) / n} \frac{\omega\left(Q_{0, i}^{*}\right) \rho\left(\omega\left(Q_{0, i}^{*}\right)\right)}{\omega(Q) \rho(\omega(Q))}
$$

and

$$
b_{3, i}:=\frac{2^{k_{0}(n+1) / n} \omega(Q) \rho(\omega(Q)) H_{0, i}}{C_{15} \omega\left(Q_{0, i}^{*}\right) \rho\left(\omega\left(Q_{0, i}^{*}\right)\right)} .
$$

Then for any $i \in\{1, \cdots, M+1\}$, from (3.61),

$$
\int_{\mathbb{R}^{n}} H_{0, i}(x) d x=0
$$

and $\operatorname{supp}\left(H_{0, i}\right) \subset Q_{0, i}^{*}$, we infer that $b_{3, i}$ is a $(\rho, q, 0)_{\omega}$-atom. By the construction of $\left\{Q_{0, i}\right\}_{i=1}^{M+1}$, we know that there exists a positive constant $C_{16}$ such that

$$
\bigcup_{i=1}^{M+1} Q_{0, i} \subset C_{16} 2^{k_{0}} Q
$$

By this, the lower $p_{0}$ property of $\Phi$, Lemma 2.2(iii) and the first inequality in (3.32), we conclude that for all $\lambda \in(0, \infty)$,

$$
\begin{aligned}
& \sum_{i=1}^{M+1} \omega\left(Q_{0, i}^{*}\right) \Phi\left(\frac{\lambda_{3, i}}{\lambda \omega\left(Q_{0, i}^{*}\right) \rho\left(\omega\left(Q_{0, i}^{*}\right)\right)}\right) \\
& \quad \lesssim \sum_{i=1}^{M+1} \omega\left(Q_{0, i}\right) \Phi\left(\frac{2^{-k_{0}(n+1) / n}}{\lambda \omega(Q) \rho(\omega(Q))}\right) \\
& \quad \lesssim \omega\left(C_{16} 2^{k_{0}} Q\right) 2^{-\frac{k_{0}(n+1) p_{0}}{n}} \Phi\left(\frac{1}{\lambda \omega(Q) \rho(\omega(Q))}\right) \\
& \quad \lesssim 2^{k_{0}\left[n q-(n+1) p_{0} / n\right]} \omega(Q) \Phi\left(\frac{1}{\lambda \omega(Q) \rho(\omega(Q))}\right) \\
& \quad \lesssim \omega(Q) \Phi\left(\frac{1}{\lambda \omega(Q) \rho(\omega(Q))}\right) .
\end{aligned}
$$

Let

$$
\widetilde{\alpha}:=\sum_{i=1}^{k_{0}} M_{k}+\sum_{k \in J_{\Omega, k_{0}}} \sum_{i} b_{k, i}+\sum_{k=1}^{k_{0}}\left(\tilde{\chi}_{k}-\tilde{\chi}_{k-1}\right) N_{k}+\sum_{i=1}^{M+1} H_{0, i} .
$$

Similar to the proof of [87, (3.55)], we see that the above equality holds in $L^{2}\left(\mathbb{R}^{n}\right)$. By the definition of $\widetilde{\alpha}$, we know that $\left.\widetilde{\alpha}\right|_{\Omega}=\alpha$. Furthermore, from (3.55), (3.56), (3.58) and (3.62), it follows that $\widetilde{\alpha} \in h_{\omega}^{\Phi}\left(\mathbb{R}^{n}\right)$ and (3.48) holds in Case 2). 
Finally, we deal with $f_{2}$. Denote by $\widetilde{K}_{R_{0}}$ the kernel of $\left(2 R_{0}^{2}+1\right) L e^{-R_{0}^{2} L}$. Then by the mean value theorem for integrals, we know that

$$
\begin{aligned}
f_{2} & =\int_{\Omega} \widetilde{K}_{R_{0}}(x, y) e^{-R_{0}^{2} L}(f)(y) d y \\
& =\sum_{Q_{k} \in \mathcal{Q}, Q_{k} \cap \Omega \neq \emptyset} \int_{Q_{k} \cap \Omega} \widetilde{K}_{R_{0}}(x, y) e^{-R_{0}^{2} L}(f)(y) d y \\
& =\sum_{Q_{k} \in \mathcal{Q}, Q_{k} \cap \Omega \neq \emptyset}\left|Q_{k} \cap \Omega\right| m_{Q_{k} \cap \Omega}\left(e^{-R_{0}^{2} L}(f)\right) \widetilde{K}_{R_{0}}\left(x, y_{k}\right),
\end{aligned}
$$

where for each $k \in \mathbb{N}, y_{k} \in Q_{k} \cap \Omega$ may depend on $x$. For each $k$, we have

$$
\widetilde{K}_{R_{0}}\left(x, y_{k}\right)=\sum_{i=0}^{\infty} \widetilde{K}_{R_{0}}\left(x, y_{k}\right) \chi_{S_{i}\left(Q_{k}\right)}:=\sum_{i=0}^{\infty} H_{k, i},
$$

where $S_{0}\left(Q_{k}\right):=2 Q_{k} \cap \Omega$ and for each $i \in \mathbb{N}$,

$$
S_{i}\left(Q_{k}\right):=\left(2^{i+1} Q_{k} \backslash 2^{i} Q_{k}\right) \cap \Omega .
$$

For each $k$, by (2.8), we see that for all $x \in \Omega$,

$$
\left|\widetilde{K}_{R_{0}}\left(x, y_{k}\right)\right| \lesssim \frac{1}{\left(1+\left|x-y_{k}\right|\right)^{n+1}} .
$$

From this, we infer that there exists a positive constant $C_{17}$ such that

$$
\left\|H_{k, 0}\right\|_{L_{\omega}^{q}\left(\mathbb{R}^{n}\right)} \lesssim\left[\omega\left(2 Q_{k}\right)\right]^{\frac{1}{q}} \lesssim C_{17} \omega\left(2 Q_{k}\right) \rho\left(\omega\left(2 Q_{k}\right)\right)\left[\omega\left(2 Q_{k}\right)\right]^{\frac{1}{q}-1}\left[\rho\left(\omega\left(2 Q_{k}\right)\right)\right]^{-1} .
$$

Thus, $\left\{C_{17} \omega\left(2 Q_{k}\right) \rho\left(\omega\left(2 Q_{k}\right)\right)\right\}^{-1} b_{k, 0}$ is a $(\rho, q, 0)_{\omega}$-atom. For all $i \in \mathbb{N}$, by (3.63), we conclude that there exists a positive constant $C_{18}$ such that

$$
\begin{aligned}
\left\|H_{k, i}\right\|_{L_{\omega}^{q}\left(\mathbb{R}^{n}\right)} \lesssim & \left\{\int_{S_{i}\left(Q_{k}\right)} \frac{\omega(x)}{\left[2^{i} l\left(Q_{k}\right)\right]^{(n+1) q}} d x\right\}^{\frac{1}{q}} \\
\lesssim & \left|2^{i} Q_{k}\right|^{-\frac{n+1}{n}}\left[\omega\left(2^{i+1} Q_{k}\right)\right]^{1 / q} \\
\lesssim & \left\{C_{18}\left|2^{i} Q_{k}\right|^{-\frac{n+1}{n}} \omega\left(2^{i+1} Q_{k}\right) \rho\left(\omega\left(2^{i+1} Q_{k}\right)\right)\right\} \\
& \times\left[\omega\left(2^{i+1} Q_{k}\right)\right]^{\frac{1}{q}-1}\left[\rho\left(\omega\left(2^{i+1} Q_{k}\right)\right)\right]^{-1},
\end{aligned}
$$

which implies that

$$
\left|2^{i} Q_{k}\right|^{(n+1) / n}\left[\omega\left(2^{i+1} Q_{k}\right) \rho\left(\omega\left(2^{i+1} Q_{k}\right)\right)\right]^{-1} H_{k, i} / C_{18}
$$

is a $(\rho, q, 0)_{\omega}$-atom. Let

$\lambda_{3, k, i}:=C_{18}\left|Q_{k} \cap \Omega\right| m_{Q_{k} \cap \Omega}\left(e^{-R_{0}^{2} L}(f)\right)\left|2^{i} Q_{k}\right|^{-(n+1) / n} \omega\left(2^{i+1} Q_{k}\right) \rho\left(\omega\left(2^{i+1} Q_{k}\right)\right)$

and

$$
a_{3, k, i}:=C_{18}^{-1}\left|2^{i} Q_{k}\right|^{\frac{n+1}{n}}\left[\omega\left(2^{i+1} Q_{k}\right) \rho\left(\omega\left(2^{i+1} Q_{k}\right)\right)\right]^{-1} H_{k, i}
$$

when $i \in \mathbb{N}$,

$$
\lambda_{3, k, 0}:=C_{17}\left|Q_{k} \cap \Omega\right| m_{Q_{k} \cap \Omega}\left(e^{-R_{0}^{2} L}(f)\right) \omega\left(2 Q_{k}\right) \rho\left(\omega\left(2 Q_{k}\right)\right)
$$

and

$$
a_{3, k, 0}:=\left\{C_{17} \omega\left(2 Q_{k}\right) \rho\left(\omega\left(2 Q_{k}\right)\right)\right\}^{-1} H_{k, 0}
$$


Then

$$
f_{2}=\sum_{k} \sum_{i=0}^{\infty} \lambda_{3, k, i} a_{3, k, i}
$$

and $\left\{a_{3, k}, i\right\}_{k, i \in \mathbb{Z}_{+}}$is a sequence of $(\rho, q, 0)_{\omega}$-atoms. From this, (3.44), (3.45) and $l\left(Q_{k}\right)=1$, Lemma 2.2 (iii) and the first inequality in (3.32), we deduce that for all $\lambda \in(0, \infty)$,

$$
\begin{aligned}
& \sum_{Q_{k} \in \mathcal{Q}, Q_{k} \cap \Omega \neq \emptyset} \sum_{i=0}^{\infty} \omega\left(2^{i+1} Q_{k}\right) \Phi\left(\frac{\left|\lambda_{3, k, i}\right|}{\lambda \omega\left(2^{i+1} Q_{k}\right) \rho\left(\omega\left(2^{i+1} Q_{k}\right)\right)}\right) \\
& \lesssim \sum_{Q_{k} \in \mathcal{Q}, Q_{k} \cap \Omega \neq \emptyset} \sum_{i=0}^{\infty} \omega\left(2^{i+1} Q_{k}\right) \Phi\left(\frac{2^{-i(n+1)} m_{Q_{k} \cap \Omega}\left(\left|e^{-R_{0}^{2} L}(f)\right|\right)}{\lambda}\right) \\
& \lesssim \sum_{\widetilde{Q}_{k} \in \mathcal{Q}_{\Omega}} \sum_{i=0}^{\infty} 2^{-i n q} 2^{-(n+1) p_{0}} \omega\left(\widetilde{Q}_{k}\right) \Phi\left(\frac{m_{\widetilde{Q}_{k} \cap \Omega}\left(\left|e^{-R_{0}^{2} L}(f)\right|\right)}{\lambda}\right) \\
& \lesssim \sum_{\widetilde{Q}_{k} \in \mathcal{Q}_{\Omega}} \omega\left(\widetilde{Q}_{k} \cap \Omega\right) \Phi\left(\frac{m_{\widetilde{Q}_{k} \cap \Omega}\left(\left|e^{-R_{0}^{2} L}(f)\right|\right)}{\lambda}\right),
\end{aligned}
$$

where for each $k, \widetilde{Q}_{k}$ is as in Definition 3.3, which, together with Lemma 3.7, implies that $f_{2} \in h_{\omega, z}^{\Phi}(\Omega)$ and hence $f_{2} \in h_{\omega, r}^{\Phi}(\Omega)$, and

$$
\begin{aligned}
\left\|f_{2}\right\|_{h_{\omega, r}^{\Phi}(\Omega)} \leq\left\|f_{2}\right\|_{h_{\omega}^{\Phi}, z}(\Omega) & \\
\lesssim \inf \{\lambda \in(0, \infty): & \sum_{\widetilde{Q}_{k} \in \mathcal{Q}_{\Omega}} \omega\left(\widetilde{Q}_{k} \cap \Omega\right) \\
& \left.\times \Phi\left(\frac{m_{\widetilde{Q}_{k} \cap \Omega}\left(\left|e^{-R_{0}^{2} L}(f)\right|\right)}{\lambda}\right) \leq 1\right\} .
\end{aligned}
$$

From (3.39), (3.49) and (3.65), we infer that $f \in h_{\omega, r}^{\Phi}(\Omega)$ and

$$
\begin{aligned}
&\|f\|_{h_{\omega, r}^{\Phi}(\Omega) \lesssim\left\|S_{h, R_{0}}^{\text {loc }}(f)\right\|_{L_{\omega}^{\Phi}(\Omega)}+\inf \{\lambda} \in(0, \infty): \sum_{\widetilde{Q}_{k} \in \mathcal{Q}_{\Omega}} \omega\left(\widetilde{Q}_{k} \cap \Omega\right) \\
&\left.\times \Phi\left(\frac{m_{\widetilde{Q}_{k} \cap \Omega}\left(\left|e^{-R_{0}^{2} L}(f)\right|\right)}{\lambda}\right) \leq 1\right\},
\end{aligned}
$$

which completes the proof of Proposition 3.13(ii).

Now we prove Proposition 3.13(iii). Let $f \in L^{2}(\Omega)$ satisfy

$$
\left\|S_{h, R_{0}}(f)\right\|_{L_{\omega}^{\Phi}(\Omega)}<\infty .
$$

By the proof of (3.39), we know that (3.39) also holds in this case. Let $f_{1}$ and $f_{2}$ be as in (3.39). Denote the zero extensions out of $\Omega$ of $f_{1}$ and $f_{2}$ respectively by $\widetilde{f}_{1}$ and $\widetilde{f}_{2}$. Similar to the proof of $f_{1} \in h_{\omega}^{\Phi}\left(\mathbb{R}^{n}\right)$ in Proposition 3.13 (i), we conclude 
that $\widetilde{f}_{1} \in h_{\omega}^{\Phi}\left(\mathbb{R}^{n}\right)$, and hence $f_{1} \in h_{\omega, z}^{\Phi}(\Omega)$ and

$$
\left\|f_{1}\right\|_{h_{\omega, z}^{\Phi}(\Omega)}=\left\|\widetilde{f}_{1}\right\|_{h_{\omega}^{\Phi}\left(\mathbb{R}^{n}\right)} \lesssim\left\|S_{h, R_{0}}^{\text {loc }}(f)\right\|_{L_{\omega}^{\Phi}(\Omega)} .
$$

Similar to the proof of $f_{2} \in h_{\omega}^{\Phi}\left(\mathbb{R}^{n}\right)$ in Proposition 3.13 (ii), we know that $\tilde{f}_{2} \in$ $h_{\omega}^{\Phi}\left(\mathbb{R}^{n}\right)$, and hence $f_{2} \in h_{\omega, z}^{\Phi}(\Omega)$ and

$$
\begin{aligned}
&\left\|f_{2}\right\|_{h_{\omega, z}^{\Phi}(\Omega)}=\left\|\tilde{f}_{2}\right\|_{h_{\omega}^{\Phi}\left(\mathbb{R}^{n}\right)} \\
& \lesssim \inf \left\{\lambda \in(0, \infty): \sum_{\widetilde{Q}_{k} \in \mathcal{Q}_{\Omega}} \omega\left(\widetilde{Q}_{k} \cap \Omega\right)\right. \\
&\left.\quad \times \Phi\left(\frac{m_{\widetilde{Q}_{k} \cap \Omega}\left(\left|e^{-R_{0}^{2} L}(f)\right|\right)}{\lambda}\right) \leq 1\right\} .
\end{aligned}
$$

Let $\tilde{f}:=\widetilde{f}_{1}+\widetilde{f}_{2}$. Then $\tilde{f}$ is the zero extension out of $\Omega$ of $f$. By the above argument, we know that $\widetilde{f} \in h_{\omega}^{\Phi}\left(\mathbb{R}^{n}\right)$ and hence $f \in h_{\omega, z}^{\Phi}(\Omega)$. Furthermore,

$$
\begin{aligned}
\|f\|_{h_{\omega}^{\Phi}, z}(\Omega) \lesssim\left\|S_{h, R_{0}}^{\mathrm{loc}}(f)\right\|_{L_{\omega}^{\Phi}(\Omega)}+\inf \{\lambda & \in(0, \infty): \sum_{\widetilde{Q}_{k} \in \mathcal{Q}_{\Omega}} \omega\left(\widetilde{Q}_{k} \cap \Omega\right) \\
& \left.\times \Phi\left(\frac{m_{\widetilde{Q}_{k} \cap \Omega}\left(\left|e^{-R_{0}^{2} L}(f)\right|\right)}{\lambda}\right) \leq 1\right\},
\end{aligned}
$$

which completes the proof of Proposition 3.13 (iii) and hence Proposition 3.13.

Now we prove Theorem 1.4 by using Propositions 3.4, 3.8, 3.12 and 3.13

Proof of Theorem 1.4. We first show Theorem 1.4(i). Let $f \in h_{\omega}^{\Phi}\left(\mathbb{R}^{n}\right) \cap L^{2}\left(\mathbb{R}^{n}\right)$, $R_{0} \in\left[\frac{1}{2}, \infty\right)$ and $Q \in \mathcal{Q}$. Then

$$
m_{Q}\left(\left|e^{-R_{0}^{2} L}(f)\right|\right) \leq \inf _{x \in Q} \mathcal{N}_{h}^{\text {loc, } 2 R_{0}}(f)(x),
$$

which implies that for all $\lambda \in(0, \infty)$,

$$
\begin{aligned}
\sum_{Q_{k} \in \mathcal{Q}} \omega\left(Q_{k}\right) \Phi\left(\frac{m_{Q_{k}}\left(\left|e^{-R_{0}^{2} L}(f)\right|\right)}{\lambda}\right) & \leq \sum_{Q_{k} \in \mathcal{Q}} \int_{Q_{k}} \Phi\left(\frac{\mathcal{N}_{h}^{\mathrm{loc}, 2 R_{0}}(f)(x)}{\lambda}\right) \omega(x) d x \\
& \lesssim \int_{\mathbb{R}^{n}} \Phi\left(\frac{\mathcal{N}_{h}^{\mathrm{loc}, 2 R_{0}}(f)(x)}{\lambda}\right) \omega(x) d x
\end{aligned}
$$

From this, together with Propositions 3.4(i), 3.8, 3.12 and 3.13(i), we deduce that

$$
\begin{aligned}
\|f\|_{h_{\omega}^{\Phi}\left(\mathbb{R}^{n}\right)} & \sim\left\|\mathcal{N}_{h}^{\text {loc, } 2 R_{0}}(f)(x)\right\|_{L_{\omega}^{\Phi}\left(\mathbb{R}^{n}\right)} \\
& \sim\left\|\widetilde{S}_{h, R_{0}}^{\text {loc }}(f)\right\|_{L_{\omega}^{\Phi}\left(\mathbb{R}^{n}\right)} \\
& +\inf \left\{\lambda \in(0, \infty): \sum_{Q_{k} \in \mathcal{Q}} \omega\left(Q_{k}\right) \Phi\left(\frac{m_{Q_{k}}\left(\left|e^{-R_{0}^{2} L}(f)\right|\right)}{\lambda}\right) \leq 1\right\}
\end{aligned}
$$




$$
\begin{aligned}
\sim & \left\|S_{h, R_{0}}^{\text {loc }}(f)\right\|_{L_{\omega}^{\Phi}\left(\mathbb{R}^{n}\right)} \\
& +\inf \left\{\lambda \in(0, \infty): \sum_{Q_{k} \in \mathcal{Q}} \omega\left(Q_{k}\right) \Phi\left(\frac{m_{Q_{k}}\left(\left|e^{-R_{0}^{2} L}(f)\right|\right)}{\lambda}\right) \leq 1\right\},
\end{aligned}
$$

which, together with the arbitrariness of $R_{0} \in\left[\frac{1}{2}, \infty\right)$ and $f \in h_{\omega}^{\Phi}\left(\mathbb{R}^{n}\right)$, implies that

$$
\begin{aligned}
h_{\omega}^{\Phi}\left(\mathbb{R}^{n}\right) \cap L^{2}\left(\mathbb{R}^{n}\right) & =h_{\mathcal{N}_{h}, \omega}^{\Phi}\left(\mathbb{R}^{n}\right) \cap L^{2}\left(\mathbb{R}^{n}\right) \\
& =h_{\widetilde{S}_{h}, \omega}^{\Phi}\left(\mathbb{R}^{n}\right) \cap L^{2}\left(\mathbb{R}^{n}\right)=h_{S_{h}, \omega}^{\Phi}\left(\mathbb{R}^{n}\right) \cap L^{2}\left(\mathbb{R}^{n}\right)
\end{aligned}
$$

with equivalent quasi-norms.

To finish the proof of Theorem 1.4 (i), we claim that $h_{\omega}^{\Phi}\left(\mathbb{R}^{n}\right) \cap L^{2}\left(\mathbb{R}^{n}\right)$ is dense in $h_{\omega}^{\Phi}\left(\mathbb{R}^{n}\right)$. We now prove the claim. For any $q \in\left(2 q_{\omega}, \infty\right]$ and $s \in \mathbb{Z}_{+}$satisfying $s \geq\left\lfloor n\left(q_{\omega} / p_{\Phi}^{-}-1\right)\right\rfloor$, denote the vector space of all finite linear combinations of $(\rho, q, s)_{\omega}$-atoms by $h_{\omega, \text { fin }}^{\rho, q, s}\left(\mathbb{R}^{n}\right)$. By Lemma 3.7 and the definition of $h_{\omega, \text { fin }}^{\rho, q, s}\left(\mathbb{R}^{n}\right)$, we know that $h_{\omega, \text { fin }}^{\rho, q}\left(\mathbb{R}^{n}\right)$ is a dense subspace of $h_{\omega}^{\Phi}\left(\mathbb{R}^{n}\right)$. For any $f \in h_{\omega, \text { fin }}^{\rho, s}\left(\mathbb{R}^{n}\right)$, let

$$
g:=\sum_{i=1}^{N} \lambda_{i} a_{i},
$$

where $N \in \mathbb{N}$, and for each $i \in\{1, \cdots, N\}, \lambda_{i} \in \mathbb{C}$ and $a_{i}$ is a $(\rho, q, s)_{\omega}$-atom. For each $i \in\{1, \cdots, N\}$, let $\operatorname{supp}\left(a_{i}\right) \subset Q_{i}$. By $q \in\left(2 q_{\omega}, \infty\right]$ and the definition of $q_{\omega}$, we see that $\omega \in A_{q / 2}\left(\mathbb{R}^{n}\right)$. From this and Hölder's inequality, we deduce that for each $i \in\{1, \cdots, N\}$,

$$
\begin{aligned}
\left\|a_{i}\right\|_{L^{2}\left(\mathbb{R}^{n}\right)} & \leq\left\|a_{i}\right\|_{L_{\omega}^{q}\left(\mathbb{R}^{n}\right)}\left\{\int_{Q_{i}}[\omega(x)]^{-\frac{2}{q-2}}\right\}^{\frac{1}{2}-\frac{1}{q}} \\
& \lesssim\left[\omega\left(Q_{i}\right)\right]^{\frac{1}{q}-1}\left[\rho\left(\omega\left(Q_{i}\right)\right)\right]^{-1} \frac{\left|Q_{i}\right|^{\frac{1}{2}}}{\left[\omega\left(Q_{i}\right)\right]^{\frac{1}{q}}} \lesssim \frac{\left|Q_{i}\right|^{\frac{1}{2}}}{\omega\left(Q_{i}\right) \rho\left(\omega\left(Q_{i}\right)\right)},
\end{aligned}
$$

which implies that $a_{i} \in L^{2}\left(\mathbb{R}^{n}\right)$. By this and the definition of $f$, we conclude that $f \in h_{\omega}^{\Phi}\left(\mathbb{R}^{n}\right) \cap L^{2}\left(\mathbb{R}^{n}\right)$, which, together with the fact that $h_{\omega, \text { fin }}^{\rho, q, s}\left(\mathbb{R}^{n}\right)$ is dense in $h_{\omega}^{\Phi}\left(\mathbb{R}^{n}\right)$, implies that $h_{\omega}^{\Phi}\left(\mathbb{R}^{n}\right) \cap L^{2}\left(\mathbb{R}^{n}\right)$ is dense in $h_{\omega}^{\Phi}\left(\mathbb{R}^{n}\right)$. Thus, the claim holds. From this, (3.64), the fact that

$$
h_{\mathcal{N}_{h}, \omega}^{\Phi}\left(\mathbb{R}^{n}\right) \cap L^{2}\left(\mathbb{R}^{n}\right), h_{\widetilde{S}_{h}, \omega}^{\Phi}\left(\mathbb{R}^{n}\right) \cap L^{2}\left(\mathbb{R}^{n}\right) \text { and } h_{S_{h}, \omega}^{\Phi}\left(\mathbb{R}^{n}\right) \cap L^{2}\left(\mathbb{R}^{n}\right)
$$

are, respectively, dense in $h_{\mathcal{N}_{h}, \omega}^{\Phi}\left(\mathbb{R}^{n}\right), h_{\widetilde{S}_{h}, \omega}^{\Phi}\left(\mathbb{R}^{n}\right)$ and $h_{S_{h}, \omega}^{\Phi}\left(\mathbb{R}^{n}\right)$, and a density argument, we deduce that the spaces $h_{\omega}^{\Phi}\left(\mathbb{R}^{n}\right), h_{\mathcal{N}_{h}, \omega}^{\Phi}\left(\mathbb{R}^{n}\right), h_{\widetilde{S}_{h}, \omega}^{\Phi}\left(\mathbb{R}^{n}\right)$ and $h_{S_{h}, \omega}^{\Phi}\left(\mathbb{R}^{n}\right)$ coincide with equivalent quasi-norms. This finishes the proof of Theorem 1.4(i).

From the definitions of the spaces $h_{\omega, r}^{\Phi}(\Omega)$ and $h_{\omega, z}^{\Phi}(\Omega)$ and the fact that $h_{\omega}^{\Phi}\left(\mathbb{R}^{n}\right) \cap$ $L^{2}\left(\mathbb{R}^{n}\right)$ is dense in $h_{\omega}^{\Phi}\left(\mathbb{R}^{n}\right)$, we deduce that $h_{\omega, r}^{\Phi}(\Omega) \cap L^{2}(\Omega)$ and $h_{\omega, z}^{\Phi}(\Omega) \cap L^{2}(\Omega)$ are, respectively, dense in $h_{\omega, r}^{\Phi}(\Omega)$ and $h_{\omega, z}^{\Phi}(\Omega)$. The remainder of the proofs of (ii) and (iii) of Theorem 1.4 is similar to that of Theorem 1.4(i). We omit the details. This finishes the proof of Theorem 1.4 


\section{Proof of Theorem 1.7}

In this section, we give the proof of Theorem 1.7 .

Proof of Theorem 1.7. We borrow some ideas from [66, 65] and [84. We prove Theorem 1.7 by using the following strategy: first, we show that (i) and (iv) are equivalent; then we prove the equivalence between (ii) and (iii); finally, we show that (ii) implies (i), which, together with the standard proof of the implication (iv) $\Longrightarrow$ (iii), completes the proof of Theorem 1.7. Thus, we divide the whole proof into the following four steps.

Step I. (i) $\Longleftrightarrow$ (iv). First we prove that (i) implies (iv). Let $f \in h_{\omega, r}^{\Phi}(\Omega)$. Then there exists $F \in h_{\omega}^{\Phi}\left(\mathbb{R}^{n}\right)$ such that $\left.F\right|_{\Omega}=f$ and

$$
\|F\|_{h_{\omega}^{\Phi}\left(\mathbb{R}^{n}\right)} \sim\|f\|_{h_{\omega, r}^{\Phi}(\Omega)} .
$$

From Lemma 3.7, it follows that there exist a sequence $\left\{a_{i}\right\}_{i}$ of $(\rho, \infty, s)_{\omega}$-atoms and $\left\{\lambda_{i}\right\}_{i} \subset \mathbb{C}$ such that $F=\sum_{i} \lambda_{i} a_{i}$ in $\mathcal{D}^{\prime}\left(\mathbb{R}^{n}\right)$ and

$$
\|F\|_{h_{\omega}^{\Phi}\left(\mathbb{R}^{n}\right)} \sim \Lambda\left(\left\{\lambda_{i} a_{i}\right\}_{i}\right),
$$

where $\Lambda\left(\left\{\lambda_{i} a_{i}\right\}_{i}\right)$ is as in Definition 3.6 .

For any $(\rho, \infty, s)_{\omega}$-atom $a$, let $\operatorname{supp}(a) \subset Q$. Since $f=\left.F\right|_{\Omega}$, we only need to consider the case that $Q \cap \Omega \neq \emptyset$. If $2 Q \subset \Omega$ and $4 Q \cap \partial \Omega=\emptyset$, then $a$ is a type (a) local $(\rho, \infty, s)_{\omega}$-atom. If $2 Q \subset \Omega$ and $4 Q \cap \partial \Omega \neq \emptyset$, then $a$ is a type $(b)$ local $(\rho, \infty, s)_{\omega}$-atom. If $2 Q \cap \partial \Omega \neq \emptyset$, by the Whitney decomposition over $Q \cap \Omega$ with $\partial \Omega$, we know that there exists a family of cubes, $\left\{Q_{j}\right\}_{j}$, with disjoint interiors such that $2 Q_{j} \subset \Omega, 4 Q_{j} \cap \partial \Omega \neq \emptyset$ and

$$
Q \cap \Omega=\bigcup_{j} Q_{j}
$$

Thus, $\left.a\right|_{\Omega}=\sum_{j} a \chi_{Q_{j}}$. For each $j$, let

$$
b_{Q_{j}}:=\frac{\omega(Q) \rho(\omega(Q))}{\omega\left(Q_{j}\right) \rho\left(\omega\left(Q_{j}\right)\right)} a \chi_{Q_{j}}
$$

and

$$
\mu_{j}:=\frac{\omega\left(Q_{j}\right) \rho\left(\omega\left(Q_{j}\right)\right)}{\omega(Q) \rho(\omega((Q))} .
$$

Then $b_{Q_{j}}$ is a type $(b) \operatorname{local}(\rho, \infty, s)_{\omega}$-atom, $\left.a\right|_{\Omega}=\sum_{j} \mu_{j} b_{Q_{j}}$ and for all $\lambda \in(0, \infty)$,

$$
\begin{aligned}
\sum_{j} \omega\left(Q_{j}\right) \Phi\left(\frac{\mu_{j}}{\lambda \omega\left(Q_{j}\right) \rho\left(\omega\left(Q_{j}\right)\right)}\right) & \leq \sum_{j} \omega\left(Q_{j}\right) \Phi\left(\frac{1}{\lambda \omega(Q) \rho(\omega(Q))}\right) \\
& \leq \omega(Q) \Phi\left(\frac{1}{\lambda \omega(Q) \rho(\omega(Q))}\right) .
\end{aligned}
$$

Thus, from the above observation, we deduce that

$$
f=\sum_{k} \mu_{1, k} b_{1, k}+\sum_{j} \mu_{2, j} b_{2, j}
$$

in $\mathcal{D}^{\prime}\left(\mathbb{R}^{n}\right)$, where $\left\{b_{1, k}\right\}_{k}$ is a sequence of type $(a)$ local $(\rho, \infty, s)_{\omega}$-atoms, $\left\{b_{2, j}\right\}_{j}$ a sequence of type $(b)$ local $(\rho, \infty, s)_{\omega}$-atoms, and $\left\{\mu_{1, k}\right\}_{k} \cup\left\{\mu_{2, j}\right\}_{j} \subset \mathbb{C}$. Moreover, 
by (4.3), we know that for all $\lambda \in(0, \infty)$,

$$
\begin{aligned}
& \sum_{k} \omega\left(Q_{1, k}\right) \Phi\left(\frac{\left|\mu_{1, k}\right|}{\lambda \omega\left(Q_{1, k}\right) \rho\left(\omega\left(Q_{1, k}\right)\right)}\right)+\sum_{j} \omega\left(Q_{2, j}\right) \Phi\left(\frac{\left|\mu_{2, j}\right|}{\lambda \omega\left(Q_{2, j}\right) \rho\left(\omega\left(Q_{2, j}\right)\right)}\right) \\
& \quad \leq \sum_{i} \omega\left(Q_{i}\right) \Phi\left(\frac{\left|\lambda_{i}\right|}{\lambda \omega\left(Q_{i}\right) \rho\left(\omega\left(Q_{i}\right)\right)}\right),
\end{aligned}
$$

where for each $k, j$ and $i, \operatorname{supp}\left(b_{1, k}\right) \subset Q_{1, k}, \operatorname{supp}\left(b_{2, j}\right) \subset Q_{2, j}$ and $\operatorname{supp}\left(a_{i}\right) \subset$ $Q_{i}$. This, combined with (4.1) and (4.2), implies that

$$
\|f\|_{h_{\omega}^{\rho, \infty, s}(\Omega)} \lesssim\|F\|_{h_{\omega}^{\rho, \infty, s}\left(\mathbb{R}^{n}\right)} \sim\|F\|_{h_{\omega}^{\Phi}\left(\mathbb{R}^{n}\right)} \sim\|f\|_{h_{\omega, r}^{\Phi}(\Omega)} .
$$

Thus, we prove that (i) implies (iv).

Now we prove that (iv) implies (i). Let $(\rho, q, s)_{\omega}$ be an admissible triplet and $f \in \mathcal{D}^{\prime}(\Omega)$ such that

$$
f=\sum_{i} \lambda_{Q_{1, i}} a_{Q_{1, i}}+\sum_{j} \lambda_{Q_{2, j}} a_{Q_{2, j}}
$$

where for each $i, a_{Q_{1, i}}$ is a type $(a)$ local $(\rho, q, s)_{\omega}$-atom supported in the cube $Q_{1, i}, \lambda_{Q_{1, i}} \in \mathbb{C}$, and for each $j, a_{Q_{2, j}}$ is a type $(b)$ local $(\rho, q, s)_{\omega}$-atom supported in the cube $Q_{2, j}$ and $\lambda_{Q_{2, j}} \in \mathbb{C}$.

To finish the proof of this case, we first construct an $F \in h_{\omega}^{\Phi}\left(\mathbb{R}^{n}\right)$ such that $\left.F\right|_{\Omega}=f$. For all $i$ and $j$, let $A_{Q_{1, i}}:=a_{Q_{1, i}}$, and if $l\left(Q_{2, j}\right) \geq 1$, let $A_{Q_{2, j}}:=a_{Q_{2, j}}$. Then both $A_{Q_{1, i}}$ and $A_{Q_{2, j}}$ are $(\rho, q, s)_{\omega}$-atoms.

Now we consider the case when $l\left(Q_{2, j}\right)<1$. It is known that for all $N \in \mathbb{N} \cup\{0\}$, there exist $\left\{\varphi_{\alpha}\right\}_{\alpha} \subset C_{c}^{\infty}(B(0,1))$ such that

$$
\int_{\mathbb{R}^{n}} x^{\beta} \varphi_{\alpha}(x) d x=\delta_{\alpha, \beta},
$$

where $\alpha, \beta \in \mathbb{Z}_{+}^{n}$ such that $|\alpha|,|\beta| \leq N$, and $\delta_{\alpha, \beta}=1$ when $\alpha=\beta$, and $\delta_{\alpha, \beta}=0$ when $\alpha \neq \beta$; see, for example, 76. Since $\Omega^{\complement}$ is unbounded, from Lemma 3.15, it follows that for any cube $Q \subset \Omega$ such that $2 Q \subset \Omega$ and $4 Q \cap \partial \Omega \neq \emptyset$, there exist $\widetilde{Q}$ and $Q^{*} \subset \mathbb{R}^{n}$ such that $Q \cup Q^{*} \subset \widetilde{Q}, Q^{*} \subset(\bar{\Omega})^{\complement}$ and

$$
l(Q)=l\left(Q^{*}\right) \sim l(\widetilde{Q}) .
$$

Let $N:=s \geq\left\lfloor n\left(\frac{q_{\omega}}{p_{\Phi}^{-}}-1\right)\right\rfloor$. For all $x \in \mathbb{R}^{n}$ and $Q_{2, j}$ satisfying $l\left(Q_{2, j}\right)<1$, let

$$
A_{Q_{2, j}}(x):=a_{Q_{2, j}}(x)-\sum_{|\alpha| \leq s} b_{\alpha} \varphi_{\alpha}\left(\frac{x-x_{Q_{2, j}^{*}}}{l\left(Q_{2, j}^{*}\right)}\right),
$$

where for each $\alpha \in \mathbb{Z}_{+}^{n}, b_{\alpha}$ is a constant which will be determined later and, for each $j, x_{Q_{2, j}^{*}}$ denotes the center of $Q_{2, j}^{*}$. For all $\alpha \in \mathbb{Z}_{+}^{n}$ with $0 \leq|\alpha| \leq s$, to show that

$$
\int_{\mathbb{R}^{n}} A_{Q_{2, j}}(x) x^{\alpha} d x=0,
$$

we set

$$
b_{\alpha}:=\frac{1}{\left[l\left(Q_{2, j}^{*}\right)\right]^{|\alpha|+n}} \int_{\mathbb{R}^{n}} a_{Q_{2, j}}(x)\left(x-x_{Q_{2, j}^{*}}\right)^{\alpha} d x .
$$


Then, for all $\alpha \in \mathbb{Z}_{+}^{n}$ with $0 \leq|\alpha| \leq s$, we see that

$$
\begin{aligned}
\int_{\mathbb{R}^{n}} A_{Q_{2, j}}(x) x^{\alpha} d x= & \int_{\mathbb{R}^{n}} a_{Q_{2, j}}(x) x^{\alpha} d x-\sum_{|\beta| \leq s} b_{\beta} \int_{\mathbb{R}^{n}} \varphi_{\beta}\left(\frac{x-x_{Q_{2, j}^{*}}}{l\left(Q_{2, j}^{*}\right)}\right) x^{\alpha} d x \\
= & \int_{\mathbb{R}^{n}} a_{Q_{2, j}}(x) x^{\alpha} d x \\
& -\sum_{|\beta| \leq s} b_{\beta}\left[l\left(Q_{2, j}^{*}\right)\right]^{n} \int_{\mathbb{R}^{n}} \varphi_{\beta}(x)\left(l\left(Q_{2, j}^{*}\right) x+x_{Q_{2, j}^{*}}\right)^{\alpha} d x=0 .
\end{aligned}
$$

Moreover, by (4.4) and Lemma 2.2(iii), we conclude that

$$
\begin{aligned}
\left\|A_{Q_{2, j}}\right\|_{L_{\omega}^{q}\left(\mathbb{R}^{n}\right)} \leq & \left\|a_{Q_{2, j},}\right\|_{L_{\omega}^{q}\left(\mathbb{R}^{n}\right)}+\sum_{|\alpha| \leq s}\left|b_{\alpha}\right|\left\|\varphi_{\alpha}\left(\frac{-x_{Q_{2, j}^{*}}}{l\left(Q_{2, j}^{*}\right)}\right)\right\|_{L_{\omega}^{q}\left(\mathbb{R}^{n}\right)} \\
\lesssim & \left\|a_{Q_{2, j},}\right\|_{L_{\omega}^{q}\left(\mathbb{R}^{n}\right)} \\
& +\sum_{|\alpha| \leq s}\left|Q_{2, j}\right|^{-\frac{n+|\alpha|}{n}}\left|\int_{\mathbb{R}^{n}} a_{Q_{2, j}}(x)\left(x-x_{Q_{2, j}^{*}}\right)^{\alpha} d x\right|\left[\omega\left(Q_{2, j}^{*}\right)\right]^{\frac{1}{q}} \\
\lesssim & {\left[\omega\left(Q_{2, j}\right)\right]^{\frac{1}{q}-1}\left[\rho\left(\omega\left(Q_{2, j}\right)\right)\right]^{-1} } \\
& +\left|Q_{2, j}\right|^{-1}\left\|a_{Q_{2, j}}\right\|_{L_{\omega}^{q}\left(\mathbb{R}^{n}\right)} \frac{\left|Q_{2, j}\right|}{\left[\omega\left(Q_{2, j}\right)\right]^{\frac{1}{q}}}\left[\omega\left(Q_{2, j}^{*}\right)\right]^{\frac{1}{q}} \\
\lesssim & {\left[\omega\left(Q_{2, j}\right)\right]^{\frac{1}{q}-1}\left[\rho\left(\omega\left(Q_{2, j}\right)\right)\right]^{-1} \sim\left[\omega\left(\widetilde{Q}_{2, j}\right)\right]^{\frac{1}{q}-1}\left[\rho\left(\omega\left(\widetilde{Q}_{2, j}\right)\right)\right]^{-1} . }
\end{aligned}
$$

Let

$$
F:=\sum_{i} \lambda_{Q_{1, i}} A_{Q_{1, i}}+\sum_{j} \lambda_{Q_{2, j}} A_{Q_{2, j}} .
$$

We then see that $F \in h_{\omega}^{\Phi}\left(\mathbb{R}^{n}\right),\left.F\right|_{\Omega}=f$ and

$$
\begin{aligned}
\|F\|_{h_{\omega}^{\Phi}\left(\mathbb{R}^{n}\right)} \lesssim & \inf \left\{\lambda \in(0, \infty): \sum_{i} \omega\left(\widetilde{Q}_{1, i}\right) \Phi\left(\frac{\left|\lambda_{Q_{1, i}}\right|}{\lambda \omega\left(\widetilde{Q}_{1, i}\right) \rho\left(\omega\left(\widetilde{Q}_{1, i}\right)\right)}\right)\right. \\
& \left.+\sum_{j} \omega\left(\widetilde{Q}_{2, j}\right) \Phi\left(\frac{\left|\lambda_{Q_{2, j}}\right|}{\lambda \omega\left(\widetilde{Q}_{2, j}\right) \rho\left(\omega\left(\widetilde{Q}_{2, j}\right)\right)}\right) \leq 1\right\} \lesssim\|f\|_{h_{\omega}^{p, q, s}(\Omega)} .
\end{aligned}
$$

From this and the definition of $h_{\omega, r}^{\Phi}(\Omega)$, we deduce that $f \in h_{\omega, r}^{\Phi}(\Omega)$ and

$$
\|f\|_{h_{\omega, r}^{\Phi}(\Omega)} \lesssim\|f\|_{h_{\omega}^{p, q, s}(\Omega)} .
$$

Thus, (vi) implies (i). This finishes the proof of Step I.

Step II. (ii) $\Longleftrightarrow$ (iii). Obviously, (ii) implies (iii). We now prove that (iii) implies (ii). Let $f \in \mathcal{D}^{\prime}(\Omega)$ such that $f_{\Omega, \varphi}^{+} \in L_{\omega}^{\Phi}(\Omega)$. Let

$$
\left[f_{\Omega, \varphi}^{+}\right]^{e}(x):= \begin{cases}f_{\Omega, \varphi}^{+}(x), & x \in \Omega, \\ 0, & x \notin \Omega .\end{cases}
$$

In what follows, for all $g \in L_{\text {loc }}^{q}\left(\mathbb{R}^{n}\right)$ with $q \in(0, \infty)$ and $x \in \mathbb{R}^{n}$, let

$$
\mathcal{M}_{q}(g)(x):=\sup _{t>0}\left[\frac{1}{|B(x, t)|} \int_{B(x, t)}|g(x)|^{q} d y\right]^{\frac{1}{q}},
$$


and denote $\mathcal{M}_{1}(g)$ simply by $\mathcal{M}(g)$, which is just the Hardy-Littlewood maximal function. Miyachi [65] proved that for all $x \in \Omega$,

$$
f_{\Omega}^{*}(x) \lesssim \mathcal{M}_{\gamma}\left(\left[f_{\Omega, \varphi}^{+}\right]^{e}\right)(x),
$$

where $\gamma \in(0,1]$ such that $\gamma<\frac{p_{\Phi}^{-}}{q_{\omega}}$. Similar to the proof of [86, (3.15)], we know that for any given $q \in\left(q_{\omega}, \infty\right)$, and all $g \in L_{\mathrm{loc}}^{1}\left(\mathbb{R}^{n}\right)$ and $\alpha \in(0, \infty)$,

$$
\omega\left(\left\{x \in \mathbb{R}^{n}: \mathcal{M}(g)(x)>2 \alpha\right\}\right) \lesssim \frac{1}{\alpha^{q}} \int_{\left\{x \in \mathbb{R}^{n}:|g(x)|>\alpha\right\}}|g(x)|^{q} \omega(x) d x .
$$

From this, it follows that for all $\alpha \in(0, \infty)$,

$$
\begin{aligned}
& \omega\left(\left\{x \in \mathbb{R}^{n}: \mathcal{M}_{\gamma}\left(\left[f_{\Omega, \varphi}^{+}\right]^{e}\right)(x)>2 \alpha\right\}\right) \\
& \quad \lesssim \frac{1}{\alpha^{\gamma q}} \int_{\left\{x \in \mathbb{R}^{n}:\left(\left[f_{\Omega, \varphi}^{+}\right]^{e}(x)\right)^{\gamma}>\frac{\alpha \gamma}{2}\right\}}\left(\left[f_{\Omega, \varphi}^{+}\right]^{e}(x)\right)^{\gamma q} \omega(x) d x \\
& \sim \sigma_{\left[f_{\Omega, \varphi}^{+}\right]^{e}}\left(\frac{\alpha}{2^{1 / \gamma}}\right)+\frac{1}{\alpha^{\gamma q}} \int_{\frac{\alpha}{2^{1 / \gamma}}}^{\infty} \gamma q s^{\gamma q-1} \sigma_{\left[f_{\Omega, \varphi}^{+}\right]^{e}}(s) d s,
\end{aligned}
$$

where and in what follows,

$$
\sigma_{\left[f_{\Omega, \varphi}^{+}\right]^{e}}(t):=\omega\left(\left\{x \in \mathbb{R}^{n}:\left[f_{\Omega, \varphi}^{+}\right]^{e}(x)>t\right\}\right) .
$$

Choose $q \in\left(q_{\omega}, \infty\right)$ and $p_{0} \in\left(0, p_{\Phi}^{-}\right)$such that $\gamma q<p_{0}$. Then $\Phi$ is of lower type $p_{0}$ and $\omega \in A_{q}\left(\mathbb{R}^{n}\right)$. From the assumption that $\Phi$ is of upper type 1 and of lower type $p_{0}$, we infer that

$$
\Phi(t) \sim \int_{0}^{t} \frac{\Phi(s)}{s} d s
$$

for all $t \in(0, \infty)$. By this, (4.5), (4.6), and the upper type 1 and the lower type $p_{0}$ properties of $\Phi$, we conclude that

$$
\begin{aligned}
\int_{\Omega} \Phi\left(f_{\Omega}^{*}(x)\right) \omega(x) d x & \sim \int_{\Omega}\left\{\int_{0}^{f_{\Omega}^{*}(x)} \frac{\Phi(t)}{t} d t\right\} \omega(x) d x \\
& \sim \int_{0}^{\infty} \frac{\Phi(t)}{t} \widetilde{\sigma}_{f_{\Omega}^{*}(t)} d t \lesssim \int_{0}^{\infty} \frac{\Phi(t)}{t} \sigma_{\mathcal{M}_{\gamma}\left(\left[f_{\Omega, \varphi}^{+}\right]^{e}\right)}(t) d t \\
\lesssim & \int_{0}^{\infty} \frac{\Phi(t)}{t}\left\{\sigma_{\left[f_{\Omega, \varphi}^{+}\right]^{e}}\left(\frac{t}{2^{1 / \gamma}}\right) d t+\frac{1}{t \gamma q} \int_{\frac{t}{2^{1 / \gamma}}}^{\infty} \gamma q s^{\gamma q-1} \sigma_{\left[f_{\Omega, \varphi}^{+}\right]^{e}}(s)\right\} d t \\
\lesssim & \int_{0}^{\infty} \frac{\Phi(t)}{t} \sigma_{\left[f_{\Omega, \varphi}^{+}\right]^{e}}(t) d t \\
& +\int_{0}^{\infty} \gamma q s^{\gamma q-1} \sigma_{\left[f_{\Omega, \varphi}^{+}\right]^{e}}(s) \Phi\left(2^{1 / \gamma} s\right)\left\{\int_{0}^{2^{1 / \gamma} s}\left(\frac{t}{2^{1 / \gamma} s}\right)^{p_{0}} \frac{1}{t^{\gamma q+1}} d t\right\} d s \\
& \sim \int_{0}^{\infty} \frac{\Phi(t)}{t} \sigma_{\left[f_{\Omega, \varphi}^{+}\right]^{e}}(t) d t \sim \int_{0}^{\infty} \frac{\Phi(t)}{t} \omega\left(\left\{x \in \Omega:\left|f_{\Omega, \varphi}^{+}(x)\right|>t\right\}\right) d t \\
& \sim \int_{\Omega} \Phi\left(f_{\Omega, \varphi}^{+}(x)\right) \omega(x) d x,
\end{aligned}
$$


where

$$
\widetilde{\sigma}_{f_{\Omega}^{*}}(t):=\omega\left(\left\{x \in \Omega: f_{\Omega}^{*}(x)>t\right\}\right) .
$$

From this and the facts that for all $\lambda \in(0, \infty)$,

$$
(f / \lambda)_{\Omega}^{*}=f_{\Omega}^{*} / \lambda \text { and }(f / \lambda)_{\Omega, \varphi}^{+}=f_{\Omega, \varphi}^{+} / \lambda
$$

we deduce that for all $\lambda \in(0, \infty)$,

$$
\int_{\Omega} \Phi\left(f_{\Omega}^{*}(x) / \lambda\right) \omega(x) d x \lesssim \int_{\Omega} \Phi\left(f_{\Omega, \varphi}^{+}(x) / \lambda\right) \omega(x) d x,
$$

which implies that

$$
\left\|f_{\Omega}^{*}\right\|_{L_{\omega}^{\Phi}(\Omega)} \lesssim\left\|f_{\Omega, \varphi}^{+}\right\|_{L_{\omega}^{\Phi}(\Omega)} .
$$

This finishes the proof of (ii) $\Longrightarrow$ (iii) and hence Step II.

Step III. (vi) $\Longrightarrow$ (iii). The proof of Step III is similar to that of Proposition $3.4(\mathrm{i})$. We omit the details.

Step IV. (ii) $\Longrightarrow$ (i). Let $f \in \mathcal{D}^{\prime}(\Omega)$ such that $f_{\Omega, \varphi}^{+} \in L_{\omega}^{\Phi}(\Omega)$. First, we show that there exists $F \in h_{\omega}^{\Phi}\left(\mathbb{R}^{n}\right)$ such that $\left.F\right|_{\Omega}=f$ and

$$
\|F\|_{h_{\omega}^{\Phi}\left(\mathbb{R}^{n}\right)} \lesssim\left\|f_{\Omega, \varphi}^{+}\right\|_{L_{\omega}^{\Phi}(\Omega)} .
$$

For the strongly Lipschitz domain $\Omega$, let the family of cubes, $\left\{Q_{k}\right\}_{k}$, be the Whitney decomposition over $\Omega$; namely, there exist a positive constant $c_{1} \in(1,5 / 4)$ and a family of cubes, $\left\{Q_{k}\right\}_{k}$, such that the $\left\{Q_{k}\right\}_{k}$ have disjoint interiors, $\bigcup_{k} Q_{k}$ $=\Omega$,

$$
\operatorname{diam}\left(Q_{k}\right) \leq \operatorname{dist}\left(Q_{k}, \Omega^{\complement}\right) \leq 4 \operatorname{diam}\left(Q_{k}\right)
$$

and $\left\{c_{1} Q_{k}\right\}_{k}$ have the bounded intersection property. In the remainder of the proof of this step, for each $k$, let $Q_{k}^{*}:=\frac{1+c_{1}}{2} Q_{k}$, and $\left\{\varphi_{k}\right\}_{k}$ be a partition of unity associated to $\left\{Q_{k}^{*}\right\}_{k}$, that is, for all $k \in \mathbb{N}, \varphi_{k} \in C_{c}^{\infty}\left(Q_{k}^{*}\right), 0 \leq \varphi_{k} \leq 1$ and $\varphi_{k} \equiv 1$ on $Q_{k}$. For each $k$, let $P_{Q_{k}} \in \mathcal{P}_{s}\left(\mathbb{R}^{n}\right)$ such that for all $P \in \mathcal{P}_{s}\left(\mathbb{R}^{n}\right)$,

$$
\left\langle f \varphi_{k}-P_{Q_{k}} \chi_{Q_{k}^{*}}, P\right\rangle=0
$$

where $\mathcal{P}_{s}\left(\mathbb{R}^{n}\right)$ denotes the linear space of polynomials in $n$ variables of degrees no more than $s$. Let

$$
g(x):= \begin{cases}f(x)-\sum_{k} \chi_{Q_{k}^{*}}(x) P_{Q_{k}}(x), & x \in \Omega, \\ 0, & x \notin \Omega .\end{cases}
$$

Now, we prove that $g \in h_{\omega}^{\Phi}\left(\mathbb{R}^{n}\right)$ and

$$
\|g\|_{h_{\omega}^{\Phi}\left(\mathbb{R}^{n}\right)} \lesssim\left\|f_{\Omega, \varphi}^{+}\right\|_{L_{\omega}^{\Phi}(\Omega)} .
$$

Recall that Miyachi [66, Lemma 1 and (3.25)] proved that for all $f \in \mathcal{D}^{\prime}(\Omega), k \in \mathbb{N}$ and $\psi \in C_{c}^{\infty}\left(Q_{k}^{*}\right)$,

$$
|\langle f, \psi\rangle| \lesssim\left|Q_{k}\right| \sum_{|\alpha| \leq s+1} \sup _{y \in \Omega}\left|\partial_{y}^{\alpha}\left(\psi\left(x_{Q_{k}}+l\left(Q_{k}\right) y\right)\right)\right| \inf _{x \in Q_{k}^{*}} f_{\Omega}^{*}(x)
$$

and

$$
\left\|P_{Q_{k}} \chi_{Q_{k}^{*}}\right\|_{L^{\infty}\left(Q_{k}^{*}\right)} \lesssim \inf _{x \in Q_{k}^{*}} f_{\Omega}^{*}(x)
$$


Let $\psi \in C_{c}^{\infty}(B(0,1))$ and

$$
\int_{\mathbb{R}^{n}} \psi(y) d y=1
$$

To prove $g \in h_{\omega}^{\Phi}\left(\mathbb{R}^{n}\right)$, we consider two cases as follows.

Case (i) $x \in \Omega$. In this case, from the definition of $g$, we deduce that (4.9) $\left|\psi_{t} * g(x)\right|$

$$
\begin{aligned}
= & \left|\sum_{k} \int_{B(x, t)}\left[f(y) \varphi_{k}(y)-P_{Q_{k}}(y) \chi_{Q_{k}^{*}}(y)\right] \psi_{t}(x-y) d y\right| \\
\leq & \sum_{\left\{k:\left|x-x_{Q_{k}}\right|<\frac{c_{1}}{2} l\left(Q_{k}\right)\right\}} \int_{B(x, t)}\left|\left[f(y) \varphi_{k}(y)-P_{Q_{k}}(y) \chi_{Q_{k}^{*}}(y)\right] \psi_{t}(x-y)\right| d y \\
& +\sum_{\left\{k:\left|x-x_{Q_{k}}\right| \geq \frac{c_{1}}{2} l\left(Q_{k}\right)\right\}} \mid \int_{Q_{k}^{*}}\left[f(y) \varphi_{k}(y)-P_{Q_{k}}(y) \chi_{Q_{k}^{*}}(y)\right] \\
& \times\left[\psi_{t}(x-y)-\sum_{|\alpha| \leq s} \partial^{\alpha}\left(\psi_{t}\right)\left(x-x_{Q_{k}}\right)\left(y-x_{Q_{k}}\right)^{\alpha}\right] d y \mid=: \mathrm{I}_{1}+\mathrm{I}_{2} .
\end{aligned}
$$

For $\mathrm{I}_{1}$, by (4.8), we know that

$$
\begin{aligned}
\mathrm{I}_{1} \lesssim & \sum_{\left\{k:\left|x-x_{Q_{k}}\right|<\frac{c_{1}}{2} l\left(Q_{k}\right)\right\}} f_{\Omega}^{*}(x) \chi_{\left\{z:\left|z-x_{Q_{k}}\right|<\frac{c_{1}}{2} l\left(Q_{k}\right)\right\}}(x) \\
& +\left\{\inf _{y \in Q_{k}^{*}} f_{\Omega}^{*}(y)\right\} \chi_{\left\{z:\left|z-x_{Q_{k}}\right|<\frac{c_{1}}{2} l\left(Q_{k}\right)\right\}}(x) .
\end{aligned}
$$

Now we estimate $\mathrm{I}_{2}$. By Taylor's remainder theorem, we conclude that

$$
\begin{aligned}
\mathrm{I}_{2} \leq & \sum_{\left\{k:\left|x-x_{Q_{k}}\right| \geq \frac{c_{1}}{2} l\left(Q_{k}\right)\right\}} \mid \int_{Q_{k}^{*}} f(y) \varphi_{k}(y) \sum_{|\alpha|=s+1} \partial^{\alpha}\left(\psi_{t}\right)(x-\xi) \\
& \times\left(y-x_{Q_{k}}\right)^{\alpha} d y\left|+\sum_{\left\{k:\left|x-x_{Q_{k}}\right| \geq \frac{c_{1}}{2} l\left(Q_{k}\right)\right\}} \int_{Q_{k}^{*}}\right| \chi_{Q_{k}^{*}}(y) P_{Q_{k}}(y) \\
& \times \sum_{|\alpha|=s+1} \partial^{\alpha}\left(\psi_{t}\right)(x-\xi)\left(y-x_{Q_{k}}\right)^{\alpha} \mid d y=: A_{t}(x)+B_{t}(x),
\end{aligned}
$$

where $\xi:=\theta y+(1-\theta) x_{Q_{k}}$ for some $\theta \in(0,1)$. We now estimate $A_{t}(x)$ and $B_{t}(x)$. From $\left|x-x_{Q_{k}}\right| \geq \frac{c_{1}}{2} l\left(Q_{k}\right)$ and $t>|x-\xi|$, we infer that

$$
t>|x-\xi| \geq\left|x-x_{Q_{k}}\right|-\theta\left|y-x_{Q_{k}}\right|>\left|x-x_{Q_{k}}\right|-\frac{1+c_{1}}{4} l\left(Q_{k}\right) .
$$

Thus,

$$
t \gtrsim\left|x-x_{Q_{k}}\right| \gtrsim l\left(Q_{k}\right) .
$$

Therefore, from (4.7) and (4.12), we deduce that

$$
A_{t}(x) \lesssim \sum_{\left\{k:\left|x-x_{Q_{k}}\right| \geq \frac{c_{1}}{2} l\left(Q_{k}\right)\right\}}\left\{\inf _{y \in Q_{k}^{*}} f_{\Omega}^{*}(y)\right\}\left|Q_{k}\right| \frac{\left[l\left(Q_{k}\right)\right]^{s+1}}{t^{n+s+1}}
$$




$$
\lesssim \sum_{\left\{k:\left|x-x_{Q_{k}}\right| \geq \frac{c_{1}}{2} l\left(Q_{k}\right)\right\}}\left\{\inf _{y \in Q_{k}^{*}} f_{\Omega}^{*}(y)\right\}\left[1+\frac{\left|x-x_{Q_{k}}\right|}{l\left(Q_{k}\right)}\right]^{-(n+s+1)} .
$$

For $B_{t}(x)$, by (4.8) and (4.12), and an estimate similar to $A_{t}(x)$, we know that

$$
B_{t}(x) \lesssim \sum_{\left\{k:\left|x-x_{Q_{k}}\right| \geq \frac{c_{1}}{2} l\left(Q_{k}\right)\right\}}\left\{\inf _{y \in Q_{k}^{*}} f_{\Omega}^{*}(y)\right\}\left[1+\frac{\left|x-x_{Q_{k}}\right|}{l\left(Q_{k}\right)}\right]^{-(n+s+1)} .
$$

Thus, combining (4.9), (4.10), (4.11), (4.13) and (4.14), we conclude that

$$
\begin{aligned}
\psi^{+}(g)(x): & =\sup _{0<t \leq 1}\left|\psi_{t} * g(x)\right| \\
\lesssim & f_{\Omega}^{*}(x)+\sum_{\left\{k:\left|x-x_{Q_{k}}\right| \geq \frac{c_{1}}{2} l\left(Q_{k}\right)\right\}}\left\{\inf _{y \in Q_{k}^{*}} f_{\Omega}^{*}(y)\right\} \\
& \times\left[1+\frac{\left|x-x_{Q_{k}}\right|}{l\left(Q_{k}\right)}\right]^{-(n+s+1)} .
\end{aligned}
$$

Case (ii) $x \notin \Omega$. In this case, we have

$$
\begin{aligned}
\psi_{t} * g(x) & =\int_{B(x, t) \cap \Omega} \sum_{k}\left[f(y) \varphi_{k}(y)-\chi_{Q_{k}^{*}}(y) P_{Q_{k}}(y)\right] \psi_{t}(x-y) d y \\
& =\int_{B(x, t) \cap \Omega} \sum_{\left\{k: Q_{k}^{*} \cap B(x, t) \neq \emptyset\right\}}\left[f(y) \varphi_{k}(y)-\chi_{Q_{k}^{*}}(y) P_{Q_{k}}(y)\right] \psi_{t}(x-y) d y .
\end{aligned}
$$

By an argument similar to Case (i), we see that

$$
\psi^{+}(g)(x) \lesssim \sum_{\left\{k: Q_{k}^{*} \cap B(x, t) \neq \emptyset\right\}} \inf _{y \in Q_{k}^{*}} f_{\Omega}^{*}(y)\left[1+\frac{\left|x-x_{Q_{k}}\right|}{l\left(Q_{k}\right)}\right]^{-(n+s+1)} .
$$

Furthermore, by $s \geq\left\lfloor n\left(q_{\omega} / p_{\Phi}^{-}-1\right)\right\rfloor$, we know that $(n+s+1) p_{\Phi}^{-}>n q_{\omega}$, which, together with the definitions of $q_{\omega}$ and $p_{\Phi}^{-}$, implies that there exist $p_{0} \in\left(0, p_{\Phi}^{-}\right)$and $q \in\left(q_{\omega}, \infty\right)$ such that $\omega \in A_{q}\left(\mathbb{R}^{n}\right), \Phi$ is of lower type $p_{0}$ and $(n+s+1) p_{0}>n q$. From this and Lemma 2.2(iii), we deduce that

$$
\int_{\mathbb{R}^{n}}\left[1+\frac{\left|x-x_{Q_{k}}\right|}{l\left(Q_{k}\right)}\right]^{-(n+s+1) p_{0}} \omega(x) d x \lesssim \omega\left(Q_{k}\right) .
$$

By this, (4.15), (4.16), the lower type $p_{0}$ property of $\Phi$ and the equivalence between (ii) and (iii) established in Step II, we conclude that

$$
\begin{aligned}
& \int_{\mathbb{R}^{n}} \Phi\left(\psi^{+}(g)(x)\right) \omega(x) d x \\
& \lesssim \int_{\Omega} \Phi\left(f_{\Omega}^{*}(x)\right) \omega(x) d x \\
& \quad+\int_{\mathbb{R}^{n}} \sum_{k} \Phi\left(\left\{\inf _{y \in Q_{k}^{*}} f_{\Omega}^{*}(y)\right\}\left[1+\frac{\left|x-x_{Q_{k}}\right|}{l\left(Q_{k}\right)}\right]^{-(n+s+1)}\right) \omega(x) d x \\
& \quad \lesssim \int_{\Omega} \Phi\left(f_{\Omega}^{*}(x)\right) \omega(x) d x+\sum_{k} \Phi\left(\inf _{y \in Q_{k}^{*}} f_{\Omega}^{*}(y)\right)
\end{aligned}
$$




$$
\begin{aligned}
& \times \int_{\mathbb{R}^{n}}\left[1+\frac{\left|x-x_{Q_{k}}\right|}{l\left(Q_{k}\right)}\right]^{-(n+s+1) p_{0}} \omega(x) d x \\
\lesssim & \int_{\Omega} \Phi\left(f_{\Omega}^{*}(x)\right) \omega(x) d x+\sum_{k} \Phi\left(\inf _{y \in Q_{k}^{*}} f_{\Omega}^{*}(y)\right) \omega\left(Q_{k}\right) \\
\lesssim & \int_{\Omega} \Phi\left(f_{\Omega}^{*}(x)\right) \omega(x) d x \lesssim \int_{\Omega} \Phi\left(f_{\Omega, \varphi}^{+}(x)\right) \omega(x) d x,
\end{aligned}
$$

which implies that $g \in h_{\omega}^{\Phi}\left(\mathbb{R}^{n}\right)$ and

$$
\|g\|_{h_{\omega}^{\Phi}\left(\mathbb{R}^{n}\right)} \lesssim\left\|f_{\Omega, \varphi}^{+}\right\|_{L_{\omega}^{\Phi}(\Omega)} .
$$

For all $x \in \mathbb{R}^{n}$, let

$$
R(x):=\sum_{k} P_{Q_{k}}(x) \chi_{Q_{k}^{*}}(x) .
$$

Let $q \in\left(q_{\omega}, \infty\right], s \in \mathbb{Z}_{+}$and $s \geq\left\lfloor n\left(\frac{q_{\omega}}{p_{\Phi}^{-}}-1\right)\right\rfloor$. For all $k \in \mathbb{N}$, let

$$
\lambda_{k}:=\frac{\omega\left(Q_{k}^{*}\right) \rho\left(\omega\left(Q_{k}^{*}\right)\right)}{\left[\omega\left(Q_{k}^{*}\right)\right]^{\frac{1}{q}}}\left\|P_{Q_{k}}\right\|_{L_{\omega}^{q}\left(Q_{k}^{*}\right)}
$$

and

Then, obviously,

$$
a_{k}:=\frac{\left[\omega\left(Q_{k}^{*}\right)\right]^{\frac{1}{q}}}{\omega\left(Q_{k}^{*}\right) \rho\left(\omega\left(Q_{k}^{*}\right)\right)} \frac{P_{Q_{k}} \chi_{Q_{k}^{*}}}{\left\|P_{Q_{k}}\right\|_{L_{\omega}^{q}\left(Q_{k}^{*}\right)}} .
$$

$$
R=\sum_{k} \lambda_{k} a_{k}
$$

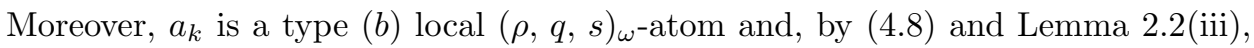
we further conclude that

$$
\begin{aligned}
& \sum_{k} \omega\left(Q_{k}^{*}\right) \Phi\left(\frac{\left|\lambda_{k}\right|}{\omega\left(Q_{k}^{*}\right) \rho\left(\omega\left(Q_{k}^{*}\right)\right)}\right) \\
& \quad \leq \sum_{k} \omega\left(Q_{k}^{*}\right) \Phi\left(\frac{\left\|P_{Q_{k}}\right\|_{L_{\omega}^{q}\left(Q_{k}^{*}\right)}}{\left[\omega\left(Q_{k}^{*}\right)\right]^{\frac{1}{q}}}\right) \leq \sum_{k} \omega\left(Q_{k}^{*}\right) \Phi\left(\left\|P_{Q_{k}}\right\|_{L^{\infty}\left(Q_{k}^{*}\right)}\right) \\
& \quad \lesssim \sum_{k} \omega\left(Q_{k}^{*}\right) \Phi\left(\inf _{y \in Q_{k}^{*}} f_{\Omega}^{*}(y)\right) \lesssim \sum_{k} \omega\left(Q_{k}\right) \Phi\left(\inf _{y \in Q_{k}^{*}} f_{\Omega}^{*}(y)\right) \\
& \quad \lesssim \int_{\Omega} \Phi\left(f_{\Omega}^{*}(y)\right) \omega(y) d y .
\end{aligned}
$$

From this, it follows that

$$
\|R\|_{h_{\omega}^{\rho, q, s}(\Omega)} \lesssim\left\|f_{\Omega}^{*}\right\|_{L_{\omega}^{\Phi}(\Omega)} .
$$

By this and the equivalence between (i) and (iv) established in Step I, we see that there exists $\widetilde{R} \in h_{\omega}^{\Phi}\left(\mathbb{R}^{n}\right)$ such that $\left.\widetilde{R}\right|_{\Omega}=R$ and

$$
\|\widetilde{R}\|_{h_{\omega}^{\Phi}\left(\mathbb{R}^{n}\right)} \lesssim\|R\|_{h_{\omega, r}^{\Phi}(\Omega)} \sim\|R\|_{h_{\omega}^{\rho, q, s}(\Omega)} \lesssim\left\|f_{\Omega}^{*}\right\|_{L_{\omega}^{\Phi}(\Omega)} \lesssim\left\|f_{\Omega, \varphi}^{+}\right\|_{L_{\omega}^{\Phi}(\Omega)} .
$$

Let $F:=g+\widetilde{R}$. By (4.17) and (4.18), we have $F \in h_{\omega}^{\Phi}\left(\mathbb{R}^{n}\right),\left.F\right|_{\Omega}=f$ and

$$
\|F\|_{h_{\omega}^{\Phi}\left(\mathbb{R}^{n}\right)} \lesssim\|g\|_{h_{\omega}^{\Phi}\left(\mathbb{R}^{n}\right)}+\|\widetilde{R}\|_{h_{\omega}^{\Phi}\left(\mathbb{R}^{n}\right)} \lesssim\left\|f_{\Omega, \varphi}^{+}\right\|_{L_{\omega}^{\Phi}(\Omega)},
$$

which completes the proof of Step VI and hence Theorem 1.7 


\section{Proof of Theorem 1.8}

In this section, we give the proof of Theorem 1.8. In what follows, we always assume that $\Omega$ is a bounded, simply connected, semiconvex domain in $\mathbb{R}^{n}$, and $\mathbb{G}_{D}$ the Dirichlet Green operator for the problem (1.1). Denote the integral kernel of $\mathbb{G}_{D}$ by $G_{D}$. We first recall the notion of semiconvex domains in $\mathbb{R}^{n}$ and some useful estimates for $G_{D}$; see [26].

Definition 5.1. (i) Let $O$ be an open set in $\mathbb{R}^{n}$. The collection of semiconvex functions on $O$ consists of continuous functions $u: O \mapsto \mathbb{R}$ with the property that there exists a positive constant $C$ such that for all $x, h \in \mathbb{R}^{n}$ with the ball $B(x,|h|) \subset O$,

$$
2 u(x)-u(x+h)-u(x-h) \leq C|h|^{2} .
$$

The best constant $C$ above is referred to as the semiconvexity constant of $u$.

(ii) A nonempty, proper open subset $\Omega$ of $\mathbb{R}^{n}$ is called semiconvex provided there exist $b, c \in(0, \infty)$ with the property that for every $x_{0} \in \partial \Omega$, there exist an $(n-1)$-dimensional affine variety $H \subset \mathbb{R}^{n}$ passing through $x_{0}$, a choice $N$ of the unit normal to $H$, and an open set

$$
\mathcal{C}:=\left\{\widetilde{x}+t N: \widetilde{x} \in H,\left|\widetilde{x}-x_{0}\right|<b,|t|<c\right\},
$$

called a coordinate cylinder near $x_{0}$ (with axis along $N$ ), such that for some semiconvex function $\varphi: H \rightarrow \mathbb{R}$ satisfying

$$
\begin{aligned}
\mathcal{C} \cap \Omega & =\mathcal{C} \cap\{\widetilde{x}+t N: \widetilde{x} \in H, t>\varphi(\widetilde{x})\}, \\
\mathcal{C} \cap \partial \Omega & =\mathcal{C} \cap\{\widetilde{x}+t N: \widetilde{x} \in H, t=\varphi(\widetilde{x})\}, \\
\mathcal{C} \cap \bar{\Omega}^{\complement} & =\mathcal{C} \cap\{\widetilde{x}+t N: \widetilde{x} \in H, t<\varphi(\widetilde{x})\},
\end{aligned}
$$

we have

$$
\varphi\left(x_{0}\right)=0 \text { and }|\varphi(\widetilde{x})|<c / 2 \text { if }\left|\widetilde{x}-x_{0}\right| \leq b .
$$

The following Lemma 5.2 was established in [36, 31]. Recall that for all $y \in \Omega$,

$$
\delta(y):=\operatorname{dist}(y, \partial \Omega) .
$$

Lemma 5.2. Let $\Omega$ and $\mathbb{G}_{D}$ be as in Theorem 1.8, Denote the integral kernel of $\mathbb{G}_{D}$ by $G_{D}$. Then there exists a positive constant $C$ such that for all $x, y \in \Omega$ with $x \neq y$,

(i) $0 \leq G_{D}(x, y) \leq C|x-y|^{2-n}$;

(ii) $\left|\nabla_{x} G_{D}(x, y)\right| \leq C|x-y|^{1-n}$;

(iii) $\left|\nabla_{x} G_{D}(x, y)\right| \leq C \delta(y)|x-y|^{-n}$;

(iv) $\left|\nabla_{y} G_{D}(x, y)\right| \leq C|x-y|^{1-n}$;

(v) $\left|\nabla_{x} \nabla_{y} G_{D}(x, y)\right| \leq C|x-y|^{-n}$.

The following Lemma 5.3 is just [26, Theorem 4.1].

Lemma 5.3. Let $\Omega$ and $\mathbb{G}_{D}$ be as in Theorem 1.8. Then the operators in (1.3), originally defined on $C^{\infty}(\bar{\Omega})$, can be extended to bounded operators on $L^{p}(\Omega)$ for $p \in(1,2]$.

Now we prove Theorem 1.8 by using Lemmas 5.2 and 5.3 and Theorem 1.7 
Proof of Theorem 1.8. We first prove Theorem 1.8(i). Fix $i, j \in\{1, \cdots, n\}$ and denote by $T$ the operator $\frac{\partial^{2} \mathbb{G}_{D}}{\partial x_{i} \partial x_{j}}$. First let $f \in h_{\omega, r}^{\Phi}(\Omega) \cap L^{2}(\Omega)$. By the assumption that $r_{\omega}>\frac{2}{2-q_{\omega}}$, we know that $\frac{r_{\omega} q_{\omega}}{r_{\omega}-1}<2$. Take $q \in\left(\frac{r_{\omega} q_{\omega}}{r_{\omega}-1}, 2\right]$ and $q_{1} \in\left(\frac{r_{\omega}}{r_{\omega}-1}, \frac{2}{q_{\omega}}\right]$ such that $\frac{q}{q_{1}}>q_{\omega}$. Then

$$
\frac{2}{2-q_{\omega}} \leq q_{1}^{\prime}<r_{\omega}
$$

where and in what follows, $\frac{1}{q_{1}}+\frac{1}{q_{1}^{\prime}}=1$. Thus, $\omega \in R H_{q_{1}^{\prime}}\left(\mathbb{R}^{n}\right)$ and $\omega \in A_{q / q_{1}}\left(\mathbb{R}^{n}\right)$. By Theorem 1.7, we conclude that

$$
f=\sum_{\text {type }(a) \text {-atoms }} \lambda_{1, k} a_{1, k}+\sum_{\text {type }(b) \text {-atoms }} \lambda_{2, m} a_{2, m},
$$

where $\left\{a_{1, k}\right\}_{k}$ and $\left\{a_{2, m}\right\}_{m}$ are respectively sequences of type $(a)$ local $(\rho, q, 0)_{\omega^{-}}$ atoms and type $(b)$ local $(\rho, q, 0)_{\omega}$-atoms, and $\left(\left\{\lambda_{1, k}\right\}_{k} \cup\left\{\lambda_{2, m}\right\}_{m}\right) \subset \mathbb{C}$. Moreover,

$$
\Lambda\left(\left\{\lambda_{1, k} a_{1, k}\right\}_{k} \cup\left\{\lambda_{2, m} a_{2, m}\right\}_{m}\right) \sim\|f\|_{h_{\omega, r}^{\Phi}(\Omega)} .
$$

To finish the proof of Theorem 1.8(i), we only need to show that for any type (a) local $(\rho, q, 0)_{\omega}$-atom or any type $(b)$ local $(\rho, q, 0)_{\omega}$-atom $a$ supported in the cube $Q_{0}$ and any $\lambda \in \mathbb{C}$,

$$
\int_{\Omega} \Phi(T(\lambda a)(x)) \omega(x) d x \lesssim \omega\left(Q_{0}\right) \Phi\left(\frac{|\lambda|}{\omega\left(Q_{0}\right) \rho\left(\omega\left(Q_{0}\right)\right)}\right) .
$$

Indeed, if (5.3) holds, then by (5.1) and the assumption that $\Phi$ is subadditive, we know that for all $\lambda \in(0, \infty)$,

$$
\begin{aligned}
\int_{\Omega} & \Phi\left(T\left(\frac{f}{\lambda}\right)(x)\right) \omega(x) d x \\
\leq & \sum_{k} \int_{\Omega} \Phi\left(T\left(\frac{\lambda_{1, k} a_{1, k}}{\lambda}\right)(x)\right) \omega(x) d x \\
& +\sum_{m} \int_{\Omega} \Phi\left(T\left(\frac{\lambda_{2, m} a_{2, m}}{\lambda}\right)(x)\right) \omega(x) d x \\
\lesssim & \sum_{k} \omega\left(Q_{1, k}\right) \Phi\left(\frac{\left|\lambda_{1, k}\right|}{\lambda \omega\left(Q_{1, k}\right) \rho\left(\omega\left(Q_{1, k}\right)\right)}\right) \\
& +\sum_{m} \omega\left(Q_{2, m}\right) \Phi\left(\frac{\left|\lambda_{2, m}\right|}{\lambda \omega\left(Q_{2, m}\right) \rho\left(\omega\left(Q_{2, m}\right)\right)}\right),
\end{aligned}
$$

where for each $k$ and $m, \operatorname{supp}\left(a_{1, k}\right) \subset Q_{1, k}$ and $\operatorname{supp}\left(a_{2, m}\right) \subset Q_{2, m}$, which, together with Theorem 1.7 and (5.2), implies that

$$
\|T(f)\|_{L_{\omega}^{\Phi}(\Omega)} \lesssim\|f\|_{h_{\omega, r}^{\Phi}(\Omega)} .
$$

From this, the fact that $h_{\omega, r}^{\Phi}(\Omega) \cap L^{2}(\Omega)$ is dense in $h_{\omega, r}^{\Phi}(\Omega)$ and a density argument, we deduce that Theorem 1.8(i) holds.

Now we prove (5.3) by considering the following three cases for $Q_{0}$.

Case i) $l\left(Q_{0}\right) \geq 1$. In this case, by

$$
\omega \in R H_{q_{1}^{\prime}}\left(\mathbb{R}^{n}\right) \cap A_{q / q_{1}}\left(\mathbb{R}^{n}\right),
$$


Jensen's inequality, Hölder's inequality, Lemma 5.3, (iii) and (v) of Lemma 2.2 the assumptions that $\Omega$ is bounded and $l\left(Q_{0}\right) \geq 1$, we conclude that

$$
\begin{aligned}
& \int_{\Omega} \Phi(T(\lambda a)(x)) \omega(x) d x \\
& \quad \leq \omega(\Omega) \Phi\left(\frac{1}{\omega(\Omega)}\left\{\int_{\Omega}|T(\lambda a)(x)|^{q_{1}} d x\right\}^{\frac{1}{q_{1}}}\left\{\int_{\Omega}[\omega(x)]^{q_{1}^{\prime}} d x\right\}^{\frac{1}{q_{1}^{\prime}}}\right) \\
& \quad \lesssim \omega(\Omega) \Phi\left(\frac{1}{|\Omega|^{\frac{1}{q_{1}}}}\|\lambda a\|_{L^{q_{1}}\left(Q_{0}\right)}\right) \lesssim \omega(\Omega) \Phi\left(\frac{|\lambda|\left|Q_{0}\right|^{\frac{1}{q_{1}}}}{|\Omega|^{\frac{1}{q_{1}}}\left[\omega\left(Q_{0}\right)\right]^{\frac{1}{q}}}\|a\|_{L_{\omega}^{q}\left(Q_{0}\right)}\right) \\
& \quad \lesssim \omega\left(Q_{0}\right) \Phi\left(\frac{|\lambda|}{\omega\left(Q_{0}\right) \rho\left(\omega\left(Q_{0}\right)\right)}\right) .
\end{aligned}
$$

Case ii) $l\left(Q_{0}\right)<1,2 Q_{0} \subset \Omega$ and $4 Q_{0} \cap \partial \Omega \neq \emptyset$. In this case, we have

$$
\begin{aligned}
\int_{\Omega} \Phi(T(\lambda a)(x)) \omega(x) d x= & \int_{4 Q_{0} \cap \Omega} \Phi(T(\lambda a)(x)) \omega(x) d x \\
& +\sum_{j=2}^{\infty} \int_{\left(2^{j+1} Q_{0} \backslash 2^{j} Q_{0}\right) \cap \Omega} \cdots=: \mathrm{I}_{1}+\mathrm{I}_{2} .
\end{aligned}
$$

Similar to the proof of (5.4), we have

$$
\mathrm{I}_{1} \lesssim \omega\left(Q_{0}\right) \Phi\left(\frac{|\lambda|}{\omega\left(Q_{0}\right) \rho\left(\omega\left(Q_{0}\right)\right)}\right)
$$

Now we deal with $\mathrm{I}_{2}$. For all $j \in \mathbb{N}$ with $j \geq 2$, let

$$
R_{j}\left(Q_{0}\right):=\left(2^{j+1} Q_{0} \backslash 2^{j} Q_{0}\right) \cap \Omega .
$$

Similar to the proof of [26, (5.34)] (or [83, p. 346, (8)]), we know that

$$
\begin{aligned}
\int_{R_{j}\left(Q_{0}\right)}|T(\lambda a)(x)| \omega(x) d x & \lesssim \frac{|\lambda| l\left(Q_{0}\right)}{\left[2^{j} l\left(Q_{0}\right)\right]^{n+1}}\|a\|_{L^{1}\left(Q_{0}\right)} \omega\left(R_{j}\left(Q_{0}\right)\right) \\
& \lesssim 2^{-j(n+1)}|\lambda| \frac{\omega\left(R_{j}\left(Q_{0}\right)\right)}{\left[\omega\left(Q_{0}\right)\right]^{\frac{1}{q}}}\|a\|_{L_{\omega}^{q}\left(Q_{0}\right)} \\
& \lesssim 2^{-j(n+1)}|\lambda| \frac{\omega\left(R_{j}\left(Q_{0}\right)\right)}{\omega\left(Q_{0}\right) \rho\left(\omega\left(Q_{0}\right)\right)}
\end{aligned}
$$

By the assumption that $n q_{\omega}<(n+1) p_{\Phi}^{-}$, we know that there exist $q_{0} \in\left(q_{\omega}, \infty\right)$ and $p_{0} \in\left(0, p_{\Phi}^{-}\right)$such that $\Phi$ is of lower type $p_{0}$ and $n q_{0}<(n+1) p_{0}$. Moreover, by the definition of $q_{\omega}$, we see that $\omega \in A_{q_{0}}\left(\mathbb{R}^{n}\right)$. Then, from Jensen's inequality, Hölder's inequality, (iii) and (v) of Lemma 2.2, the lower type $p_{0}$ property of $\Phi$, (5.7) and $n q_{0}<(n+1) p_{0}$, it follows that

$$
\begin{aligned}
\mathrm{I}_{2} & \leq \sum_{j=2}^{\infty} \omega\left(R_{j}\left(Q_{0}\right)\right) \Phi\left(\frac{1}{\omega\left(R_{j}\left(Q_{0}\right)\right)} \int_{R_{j}\left(Q_{0}\right)}|T(\lambda a)(x)| \omega(x) d x\right) \\
& \lesssim \sum_{j=2}^{\infty} \omega\left(R_{j}\left(Q_{0}\right)\right) \Phi\left(2^{-k(n+1)} \frac{|\lambda|}{\omega\left(Q_{0}\right) \rho\left(\omega\left(Q_{0}\right)\right)}\right) \\
& \lesssim \sum_{j=2}^{\infty} 2^{-j\left[(n+1) p_{0}-n q_{0}\right]} \omega\left(Q_{0}\right) \Phi\left(\frac{|\lambda|}{\omega\left(Q_{0}\right) \rho\left(\omega\left(Q_{0}\right)\right)}\right)
\end{aligned}
$$




$$
\lesssim \omega\left(Q_{0}\right) \Phi\left(\frac{|\lambda|}{\omega\left(Q_{0}\right) \rho\left(\omega\left(Q_{0}\right)\right)}\right) .
$$

Thus, by (5.5), (5.6) and (5.8), we conclude that (5.3) holds in this case.

Case iii) $l\left(Q_{0}\right)<1$ and $4 Q_{0} \subset \Omega$. In this case, we have

$$
\begin{aligned}
\int_{\Omega} & \Phi(T(\lambda a)(x)) \omega(x) d x \\
& =\int_{2 Q_{0}} \Phi(T(\lambda a)(x)) \omega(x) d x+\sum_{j=1}^{\infty} \int_{\left(2^{j+1} Q_{0} \backslash 2^{j} Q_{0}\right) \cap \Omega} \cdots=: \mathrm{J}_{0}+\sum_{j=1}^{\infty} \mathrm{J}_{j} .
\end{aligned}
$$

For $j \in\{0,1,2\}$, similar to the estimate of (5.6), we know that

$$
\mathrm{J}_{j} \lesssim \omega\left(Q_{0}\right) \Phi\left(\frac{|\lambda|}{\omega\left(Q_{0}\right) \rho\left(\omega\left(Q_{0}\right)\right)}\right) .
$$

Now we deal with $\mathrm{J}_{j}$ for $j \in \mathbb{N}$ with $j \geq 3$. Pick $\varphi \in C_{c}^{\infty}\left(\mathbb{R}_{+}\right)$satisfying $\varphi(t) \equiv 0$ if $t \leq \frac{1}{2}$ or $t \geq 4$, and $\varphi(t) \equiv 1$ if $1 \leq t \leq 2$. Furthermore, for each $j \geq 3$, let

$$
\varphi_{j}(x):=\varphi\left(\left|x-x_{Q_{0}}\right| /\left(2^{j} l\left(Q_{0}\right)\right)\right)
$$

for all $x \in \mathbb{R}^{n}$, where $x_{Q_{0}}$ denotes the center of $Q_{0}$. Similar to the proof of $[26$, p. 49, (5.29)], we have

$$
\begin{aligned}
\int_{R_{j}\left(Q_{0}\right)}|T(\lambda a)(x)| \omega(x) d x \lesssim & \int_{R_{j}\left(Q_{0}\right)}\left|\mathbb{G}_{D}(\lambda a)(x) \Delta \varphi_{j}(x)\right| \omega(x) d x \\
& +\int_{R_{j}\left(Q_{0}\right)}\left|\nabla_{x} \mathbb{G}_{D}(\lambda a)(x) \cdot \nabla \varphi_{j}(x)\right| \omega(x) d x
\end{aligned}
$$

In this case, we know that $a$ is a type local $(a)(\rho, q, 0)_{\omega}$-atom, and hence

$$
\int_{\Omega} a(x) d x=0 .
$$

From this, Lemma 5.2 (iv) and Hölder's inequality, it follows that for all $x \in R_{j}\left(Q_{0}\right)$,

$$
\begin{aligned}
\left|\mathbb{G}_{D}(\lambda a)(x)\right| & =\left|\lambda \int_{Q_{0}} \mathbb{G}_{D}(x, y) a(y) d y\right| \\
& =\left|\lambda \int_{Q_{0}}\left[\mathbb{G}_{D}(x, y)-\mathbb{G}_{D}\left(x, x_{Q_{0}}\right)\right] a(y) d y\right| \\
& \leq|\lambda| \int_{Q_{0}}\left|\nabla_{y} \mathbb{G}_{D}\left(x, y_{1}\right)\right|\left|y-x_{Q_{0}}\right||a(y)| d y \\
& \lesssim|\lambda| \int_{Q_{0}} \frac{\left|y-x_{Q_{0}}\right|}{\left|x-y_{1}\right|^{n-1}}|a(y)| d y \lesssim \frac{|\lambda| l\left(Q_{0}\right)}{\left|x-x_{Q_{0}}\right|^{n-1}}\|a\|_{L^{1}\left(\mathbb{R}^{n}\right)} \\
& \lesssim \frac{|\lambda| l\left(Q_{0}\right)}{\left|x-x_{Q_{0}}\right|^{n-1}} \frac{\left|Q_{0}\right|}{\left[\omega\left(Q_{0}\right)\right]^{\frac{1}{q}}}\|a\|_{L_{\omega}^{q}\left(Q_{0}\right)},
\end{aligned}
$$

where $y_{1} \in Q_{0}$ depends on $y$ and $x_{Q_{0}}$, and in the penultimate inequality we used the fact that $\left|x-y_{1}\right| \sim\left|x-x_{Q_{0}}\right|$, which, together with the fact that for all $x \in \mathbb{R}^{n}$, $\left|\Delta \varphi_{j}(x)\right| \lesssim\left[2^{j} l\left(Q_{0}\right)\right]^{-2}$, implies that

$$
\int_{\Omega}\left|\mathbb{G}_{D}(\lambda a)(x) \Delta \varphi_{j}(x)\right| \omega(x) d x \lesssim 2^{-j(n+1)} \frac{|\lambda| \omega\left(R_{j}\left(Q_{0}\right)\right)}{\omega\left(Q_{0}\right) \rho\left(\omega\left(Q_{0}\right)\right)} .
$$


Moreover, by the fact that $\int_{Q_{0}} a(x) d x=0$, Lemma $5.2(\mathrm{v})$ and Hölder's inequality, we know that for all $x \in R_{j}\left(Q_{0}\right)$,

$$
\begin{aligned}
\left|\nabla_{x} \mathbb{G}_{D}(\lambda a)(x)\right| & =\left|\lambda \int_{Q_{0}} \nabla_{x} G_{D}(x, y) a(y) d y\right| \\
& =\left|\lambda \int_{Q_{0}}\left[\nabla_{x} G_{D}(x, y)-\nabla_{x} G_{D}\left(x, x_{Q_{0}}\right)\right] a(y) d y\right| \\
& \leq|\lambda| \int_{Q_{0}}\left|y-x_{Q_{0}}\right| \sup _{y_{1} \in Q_{0}}\left|\nabla_{x} \nabla_{y} G_{D}\left(x, y_{1}\right)\right||a(y)| d y \\
& \lesssim \frac{|\lambda| l\left(Q_{0}\right)}{\left|x-x_{Q_{0}}\right|^{n}}\|a\|_{L^{1}\left(Q_{0}\right)} \lesssim \frac{|\lambda|\left[l\left(Q_{0}\right)\right]^{n+1}\|a\|_{L_{\omega}^{q}\left(Q_{0}\right)}}{\left|x-x_{Q_{0}}\right|^{n}\left[\omega\left(Q_{0}\right)\right]^{\frac{1}{q}}}
\end{aligned}
$$

which, together with the fact that for all $x \in \mathbb{R}^{n},\left|\nabla \varphi_{j}(x)\right| \lesssim\left[2^{j} l\left(Q_{0}\right)\right]^{-1}$, implies that

$$
\int_{\Omega}\left|\nabla \mathbb{G}_{D}(\lambda a)(x) \cdot \nabla \varphi_{j}(x)\right| \omega(x) d x \lesssim 2^{-j(n+1)} \frac{|\lambda| \omega\left(R_{j}\left(Q_{0}\right)\right)}{\omega\left(Q_{0}\right) \rho\left(\omega\left(Q_{0}\right)\right)} .
$$

Thus, by (5.11), (5.12) and (5.13), we conclude that for each $j \in \mathbb{N}$ with $j \geq 3$,

$$
\int_{R_{j}\left(Q_{0}\right)}|T(\lambda a)(x)| \omega(x) d x \lesssim 2^{-j(n+1)} \frac{|\lambda| \omega\left(R_{j}\left(Q_{0}\right)\right)}{\omega\left(Q_{0}\right) \rho\left(\omega\left(Q_{0}\right)\right)},
$$

which, together with Jensen's inequality, (iii) and (v) of Lemma 2.2. the lower type $p_{0}$ property of $\Phi$ and $n q_{0}<(n+1) p_{0}$, implies that

$$
\begin{aligned}
\sum_{j=3}^{\infty} \mathrm{J}_{j} & \leq \sum_{j=3}^{\infty} \omega\left(R_{j}\left(Q_{0}\right)\right) \Phi\left(\frac{1}{\omega\left(R_{j}\left(Q_{0}\right)\right)} \int_{R_{j}\left(Q_{0}\right)}|T(\lambda a)(x)| \omega(x) d x\right) \\
& \lesssim \sum_{j=3}^{\infty} \omega\left(R_{j}\left(Q_{0}\right)\right) \Phi\left(2^{-j(n+1)} \frac{|\lambda|}{\omega\left(Q_{0}\right) \rho\left(\omega\left(Q_{0}\right)\right)}\right) \\
& \lesssim \sum_{j=3}^{\infty} 2^{-j\left[(n+1) p_{0}-n q_{0}\right]} \omega\left(Q_{0}\right) \Phi\left(\frac{|\lambda|}{\omega\left(Q_{0}\right) \rho\left(\omega\left(Q_{0}\right)\right)}\right) \\
& \lesssim \omega\left(Q_{0}\right) \Phi\left(\frac{|\lambda|}{\omega\left(Q_{0}\right) \rho\left(\omega\left(Q_{0}\right)\right)}\right) .
\end{aligned}
$$

This, combined with (5.9) and (5.10), implies that (5.3) holds in this case and hence completes the proof of Theorem 1.8(i). let

Now we prove Theorem 1.8(ii). We borrow some ideas from [26] and [83]. First

$$
f \in h_{\omega, r}^{\Phi}(\Omega) \cap L^{2}(\Omega) .
$$

By the assumption that $r_{\omega}>\frac{2}{2-q_{\omega}}$ and the definition of $q_{\omega}$, we know that there exists $q_{2} \in\left(q_{\omega}, 2\right)$ such that

$$
\frac{2}{2-q_{2}}<r_{\omega}
$$

Then $\left(\frac{2}{q_{2}}\right)^{\prime}<r_{\omega}$ and hence $\omega \in A_{\left(2 / q_{2}\right)^{\prime}}\left(\mathbb{R}^{n}\right)$. Take $q_{3} \in\left(2 q_{\omega}, \infty\right)$. Then $\omega \in$ $A_{q_{3} / 2}\left(\mathbb{R}^{n}\right)$. Then by Theorem 1.7. we know that there exist a sequence $\left\{a_{1, k}\right\}_{k}$ of 
type $(a)$ local $\left(\rho, q_{2}, 0\right)_{\omega}$-atoms, a sequence $\left\{a_{2, m}\right\}_{m}$ of type $(b)$ local $\left(\rho, q_{3}, 0\right)_{\omega^{-}}$ atoms and $\left(\left\{\lambda_{1, k}\right\}_{k} \cup\left\{\lambda_{2, m}\right\}_{m}\right) \subset \mathbb{C}$ such that (5.1) and (5.2) hold. Moreover, by Lemma 5.3. we see that

$$
T(f)=\sum_{\text {type }(a) \text { atoms }} \lambda_{1, k} T\left(a_{1, k}\right)+\sum_{\text {type }(b) \text { atoms }} \lambda_{2, m} T\left(a_{2, m}\right) \text { in } L^{2}(\Omega) .
$$

To finish the proof of Theorem 1.8(ii), we only need to show that for all type $(a)$ local $\left(\rho, q_{2}, 0\right)_{\omega}$-atoms or type $(b)$ local $\left(\rho, q_{2}, 0\right)_{\omega}$-atoms $a$ supported in the cube $Q_{0}$ and all $\lambda \in \mathbb{C} \backslash\{0\}$, there exist a sequence $\left\{b_{s}\right\}_{s}$ of type $(a)$ local $\left(\rho, q_{1}, 0\right)$-atoms and type $(b)$ local $\left(\rho, q_{1}, 0\right)$-atoms, and $\left\{\mu_{s}\right\}_{s} \subset \mathbb{C}$ such that

$$
T(\lambda a)=\sum_{s} \mu_{s} b_{s}
$$

and for all $\mu \in(0, \infty)$,

$$
\sum_{s} \omega\left(Q_{s}\right) \Phi\left(\frac{\left|\mu_{s}\right|}{\mu \omega\left(Q_{s}\right) \rho\left(\omega\left(Q_{s}\right)\right)}\right) \lesssim \omega\left(Q_{0}\right) \Phi\left(\frac{|\lambda|}{\mu \omega\left(Q_{0}\right) \rho\left(\omega\left(Q_{0}\right)\right)}\right),
$$

where for each $s, \operatorname{supp}\left(b_{s}\right) \subset Q_{s}$.

Indeed, if (5.14) and (5.15) hold, then for each $k$ and $m$, there exist sequences $\left\{b_{1, k, s}\right\}_{k, s}$ and $\left\{b_{2, m, s}\right\}_{m, s}$ of type $(a)$ local $\left(\rho, q_{1}, 0\right)$-atoms and type $(b)$ local $\left(\rho, q_{1}, 0\right)$-atoms, and $\left\{\mu_{1, k, s}\right\}_{k, s} \cup\left\{\mu_{2, m, s}\right\}_{m, s} \subset \mathbb{C}$ such that

$$
T(f)=\sum_{k, s} \lambda_{1, k} \mu_{1, k, s} b_{1, k, s}+\sum_{m, s} \lambda_{2, m} \mu_{2, m, s} b_{2, m, s},
$$

and for all $\lambda \in(0, \infty)$,

$$
\begin{aligned}
& \sum_{k, s} \omega\left(Q_{1, k, s}\right) \Phi\left(\frac{\left|\lambda_{1, k} \mu_{1, k, s}\right|}{\lambda \omega\left(Q_{1, k, s}\right) \rho\left(\omega\left(Q_{1, k, s}\right)\right)}\right) \\
& \quad+\sum_{m, s} \omega\left(Q_{2, m, s}\right) \Phi\left(\frac{\left|\lambda_{2, m} \mu_{2, m, s}\right|}{\lambda \omega\left(Q_{2, m, s}\right) \rho\left(\omega\left(Q_{2, m, s}\right)\right)}\right) \\
& \lesssim \sum_{k} \omega\left(Q_{1, k}\right) \Phi\left(\frac{\left|\lambda_{1, k}\right|}{\lambda \omega\left(Q_{1, k}\right) \rho\left(\omega\left(Q_{1, k}\right)\right)}\right) \\
& \quad+\sum_{m} \omega\left(Q_{2, m}\right) \Phi\left(\frac{\left|\lambda_{2, m}\right|}{\lambda \omega\left(Q_{2, m}\right) \rho\left(\omega\left(Q_{2, m}\right)\right)}\right)
\end{aligned}
$$

where for each $k, m, s, \operatorname{supp}\left(a_{1, k}\right) \subset Q_{1, k}, \operatorname{supp}\left(a_{2, m}\right) \subset Q_{2, m}, \operatorname{supp}\left(b_{1, k, s}\right) \subset$ $Q_{1, k, s}$ and $\operatorname{supp}\left(b_{2, m, s}\right) \subset Q_{2, m, s}$, which, together with Theorem 1.7 and (5.2), implies that $T(f) \in h_{\omega, r}^{\Phi}(\Omega)$ and

$$
\|T(f)\|_{h_{\omega, r}^{\Phi}(\Omega)} \lesssim\|f\|_{h_{\omega, r}^{\Phi}(\Omega)} .
$$

From this, the fact that $h_{\omega, r}^{\Phi}(\Omega) \cap L^{2}(\Omega)$ is dense in $h_{\omega, r}^{\Phi}(\Omega)$ and a density argument, we deduce Theorem 1.8 (ii).

Now we prove (5.14) and (5.15) by considering the following two cases for $a$.

Case i) $a$ is a type (a) local $\left(\rho, q_{3}, 0\right)_{\omega}$-atom. Recall that the standard fundamental solution of the Laplace operator

$$
\Delta:=\sum_{i=1}^{n} \frac{\partial^{2}}{\partial x_{i}^{2}}
$$


on $\mathbb{R}^{n}(n \geq 2)$ is given by

$$
\Gamma(x):= \begin{cases}\frac{1}{2 \pi} \ln |x|, & \text { when } n=2, \\ \frac{c_{n}}{|x|^{n-2}}, & \text { when } n \geq 3,\end{cases}
$$

where $c_{n}:=\left[(n-2) \omega_{n}\right]^{-1}$ and $\omega_{n}$ denotes the area of the unit sphere in $\mathbb{R}^{n}$. This allows us to solve the Poisson problem for the Laplacian in the whole space via integral operators. Indeed, as is well known, the Newtonian potential

$$
E(f)(x):=\int_{\Omega} \Gamma(x-y) f(y) d y, x \in \Omega,
$$

satisfies $\Delta E(f)=f$ in $\Omega$ when $f \in C^{\infty}(\Omega)$.

For each $y \in \Omega$, let $U(\cdot, y)$ be the solution of the Dirichlet problem

$$
\begin{cases}\Delta U(\cdot, y)=0, & \text { in } \Omega, \\ U(x, y)=\Gamma(x-y), & \text { for } x \in \partial \Omega .\end{cases}
$$

Then the Green function $G_{D}$ for $\Delta_{D}$ on $\Omega$ (which is the integral kernel of the Dirichlet Green potential $\mathbb{G}_{D}$ ) can be expressed as

$$
G_{D}(x, y)=\Gamma(x-y)-U(x, y), x, y \in \Omega, x \neq y .
$$

As a consequence, the solution of the inhomogeneous Dirichlet problem (1.1) is given by

$$
\begin{aligned}
\mathbb{G}_{D}(f)(x) & =\int_{\Omega} G_{D}(x, y) f(y) d y \\
& =\int_{\Omega} \Gamma(x-y) f(y) d y-\int_{\Omega} U(x, y) f(y) d y \\
& =: E(f)(x)-U(f)(x),
\end{aligned}
$$

where $f \in C^{\infty}(\Omega)$ and $x \in \Omega$.

By abuse of notation, we denote by $a$ the zero extension of $a$ out of $\Omega$. Then similar to the proof of [86, Theorem 8.2], we know that

$$
\frac{\partial^{2} E(\lambda a)}{\partial x_{i} \partial x_{j}} \in h_{\omega}^{\Phi}\left(\mathbb{R}^{n}\right) \text { and }\left\|\frac{\partial^{2} E(\lambda a)}{\partial x_{i} \partial x_{j}}\right\|_{h_{\omega}^{\Phi}\left(\mathbb{R}^{n}\right)} \lesssim\|\lambda a\|_{h_{\omega}^{\Phi}\left(\mathbb{R}^{n}\right)},
$$

which, together with the definition of $h_{\omega, r}^{\Phi}(\Omega)$, implies that

$$
\left\|\frac{\partial^{2} E(\lambda a)}{\partial x_{i} \partial x_{j}}\right\|_{h_{\omega, r}^{\Phi}(\Omega)} \leq\left\|\frac{\partial^{2} E(\lambda a)}{\partial x_{i} \partial x_{j}}\right\|_{h_{\omega}^{\Phi}\left(\mathbb{R}^{n}\right)} \lesssim\|\lambda a\|_{h_{\omega}^{\Phi}\left(\mathbb{R}^{n}\right)} .
$$

For $i, j \in\{1, \cdots, n\}$, let

$$
H_{i, j}(\lambda a):=\frac{\partial^{2} U(\lambda a)}{\partial x_{i} \partial x_{j}} .
$$

From (5.17) and (5.18), we infer that $H_{i, j}(\lambda a)$ is a harmonic function in $\Omega$. Thus, an application of Theorem 1.7 in which we take the function $\varphi$ to be radial, yields, on account of the mean value property for harmonic functions, that for all $x \in \Omega$,

$$
\begin{aligned}
\left(H_{i, j}(\lambda a)\right)_{\Omega, \varphi}^{+}(x) & =\sup _{0<t<\delta(x) / c_{0}}\left|\int_{\Omega} H_{i, j}(\lambda a)(y) \varphi_{t}(x-y) d y\right| \\
& =\left|H_{i, j}(\lambda a)(x)\right| .
\end{aligned}
$$


Similar to the proof of Theorem 1.8(i), we know that

$$
\left\|\frac{\partial^{2} E(\lambda a)}{\partial x_{i} \partial x_{j}}\right\|_{L_{\omega}^{\Phi}\left(\mathbb{R}^{n}\right)} \lesssim\|\lambda a\|_{h_{\omega}^{\Phi}\left(\mathbb{R}^{n}\right)} .
$$

By this, (5.20), Theorem 1.7 and Theorem 1.8(i), we conclude that

$$
\begin{aligned}
\left\|H_{i, j}(\lambda a)\right\|_{h_{\omega, r}^{\Phi}(\Omega)} & =\left\|\left(H_{i, j}(\lambda a)\right)_{\Omega, \varphi}^{+}\right\|_{L_{\omega}^{\Phi}(\Omega)}=\left\|H_{i, j}(\lambda a)\right\|_{L_{\omega}^{\Phi}(\Omega)} \\
& \lesssim\left\|\frac{\partial^{2} E(\lambda a)}{\partial_{i} x_{i} \partial_{j} x_{j}}\right\|_{L_{\omega}^{\Phi}(\Omega)}+\left\|\frac{\partial^{2} \mathbb{G}_{D}(\lambda a)}{\partial x_{i} \partial x_{j}}\right\|_{L_{\omega}^{\Phi}(\Omega)} \lesssim\|\lambda a\|_{h_{\omega}^{\Phi}\left(\mathbb{R}^{n}\right)},
\end{aligned}
$$

which, together with (5.19), implies that $T(\lambda a) \in h_{\omega, r}^{\Phi}(\Omega)$ and

$$
\|T(\lambda a)\|_{h_{\omega, r}^{\Phi}(\Omega)} \lesssim\|\lambda a\|_{h_{\omega}^{\Phi}\left(\mathbb{R}^{n}\right)} .
$$

From this and Theorem 1.7, we infer that (5.14) and (5.15) hold in this case.

Case ii) $a$ is a type $(b)$ local $\left(\rho, q_{3}, 0\right)_{\omega}$-atom. Let

$$
R_{k}\left(Q_{0}\right):=\left(2^{k+1} Q_{0} \backslash 2^{k} Q_{0}\right) \cap \Omega
$$

when $k \geq 3, R_{0}\left(Q_{0}\right):=8 Q_{0} \cap \Omega$ and

$$
J_{\Omega}:=\left\{k \in \mathbb{N}: k \geq 3,\left|R_{k}\left(Q_{0}\right)\right|>0\right\} .
$$

For all $k \in \mathbb{N} \cup\{0\}$, let $\chi_{k}:=\chi_{R_{k} Q_{0}}, \tilde{\chi}_{k}:=\frac{\chi_{k}}{\left|R_{k}\left(Q_{0}\right)\right|}$ and

$$
m_{k}:=\int_{R_{k}\left(Q_{0}\right)} T(\lambda a) d x .
$$

Then, we have

$$
T(\lambda a)=T(\lambda a) \chi_{0}+\sum_{k \in J_{\Omega}} T(\lambda a) \chi_{k} .
$$

Let $\widetilde{Q}_{0} \subset \mathbb{R}^{n}$ satisfy $x_{\widetilde{Q}_{0}} \in \Omega^{\complement}, l\left(\widetilde{Q}_{0}\right)=l\left(Q_{0}\right)$ and $\operatorname{dist}\left(Q_{0}, \widetilde{Q}_{0}\right) \sim l\left(Q_{0}\right)$, which implies that there exists $Q_{0}^{*} \subset \mathbb{R}^{n}$ such that $\left(8 Q_{0} \cup \widetilde{Q}_{0}\right) \subset Q_{0}^{*}$ and $l\left(Q_{0}^{*}\right) \sim l\left(Q_{0}\right)$. Moreover, let

$$
H_{0}:=T(\lambda a) \chi_{0}-\frac{1}{\left|\widetilde{Q}_{0} \cap \Omega\right|}\left\{\int_{R_{0}\left(Q_{0}\right)} T(\lambda a)(y) d y\right\} \chi_{\widetilde{Q}_{0} \cap \Omega^{\mathrm{C}}} .
$$

We have

$$
\int_{\mathbb{R}^{n}} H_{0}(x) d x=0 \quad \text { and } \quad \operatorname{supp}\left(H_{0}\right) \subset Q_{0}^{*} .
$$

From $\omega \in R H_{\left(2 / q_{2}\right)^{\prime}}\left(\mathbb{R}^{n}\right) \cap A_{q_{3} / 2}\left(\mathbb{R}^{n}\right)$, Hölder's inequality and Lemma 5.3, it follows that

$$
\begin{aligned}
\left\|H_{0}\right\|_{L_{\omega}^{q_{2}}\left(\mathbb{R}^{n}\right)} & \lesssim\|T(\lambda a)\|_{L_{\omega}^{q_{2}}\left(R_{0}\left(Q_{0}\right)\right)} \\
& \leq\left\{\int_{R_{0}\left(Q_{0}\right)}|T(\lambda a)(x)|^{2} d x\right\}^{\frac{1}{2}}\left\{\left(\int_{R_{0}\left(Q_{0}\right)}[\omega(x)]^{\left(\frac{2}{q_{2}}\right)^{\prime}} d x\right)^{\frac{1}{\left(\frac{2}{q_{2}}\right)^{\prime}}}\right\}^{\frac{1}{q_{2}}} \\
& \leq\|\lambda a\|_{L^{2}(\Omega)}\left\{\frac{\omega\left(R_{0}\left(Q_{0}\right)\right.}{\left|Q_{0}\right|^{q_{2} / 2}}\right\}^{\frac{1}{q_{2}}} \leq\|\lambda a\|_{L_{\omega}^{q_{3}}(\Omega)} \frac{\left|Q_{0}\right|^{\frac{1}{2}}}{\left[\omega\left(Q_{0}\right)\right]^{\frac{1}{q_{3}}}} \frac{\left[\omega\left(R_{0}\left(Q_{0}\right)\right)\right]^{\frac{1}{q_{2}}}}{\left|Q_{0}\right|^{\frac{1}{2}}} \\
& \lesssim|\lambda|\left[\omega\left(Q_{0}\right)\right]^{\frac{1}{q_{2}}-1}\left[\rho\left(\omega\left(Q_{0}\right)\right)\right]^{-1} \sim|\lambda|\left[\omega\left(Q_{0}^{*}\right)\right]^{\frac{1}{q_{2}}-1}\left[\rho\left(\omega\left(Q_{0}^{*}\right)\right)\right]^{-1} .
\end{aligned}
$$


Thus, there exists a positive constant $\widetilde{C}$ such that $\frac{H_{0}}{\widetilde{C}|\lambda|}$ is a $\left(\rho, q_{1}, 0\right)_{\omega}$-atom.

If $k \in J_{\Omega}$, by Fact 1 in the proof of Proposition 3.13, we know that there exist $\left\{Q_{k, j}\right\}_{j} \subset \mathbb{R}^{n}$ such that for all $j, 2 Q_{k, j} \subset \Omega, 4 Q_{k, j} \cap \partial \Omega \neq \emptyset$ and

$$
R_{k}\left(Q_{0}\right)=\bigcup_{j} Q_{k, j}
$$

From Lemma 3.15] it follows that for each $k$ and $j$, there exists $\widetilde{Q}_{k, j} \subset \Omega^{\complement}$ such that $l\left(\widetilde{Q}_{k, j}\right)=l\left(Q_{k, j}\right)$ and $\operatorname{dist}\left(\widetilde{Q}_{k, j}, Q_{k, j}\right) \sim l\left(Q_{k, j}\right)$. For each $k$ and $j$, take cube $Q_{k, j}^{*} \subset \mathbb{R}^{n}$ such that

$$
Q_{k, j} \cup \widetilde{Q}_{k, j} \subset Q_{k, j}^{*} \text { and } l\left(Q_{k, j}^{*}\right) \sim l\left(Q_{k, j}\right) .
$$

By this, Hölder's inequality, Lemma 5.3 , (iii) and (v) of Lemma 2.2 we see that

$$
\begin{aligned}
&\left\|H_{k, i}\right\|_{L_{\omega}^{q_{2}}\left(\mathbb{R}^{n}\right)} \lesssim\|T(\lambda a)\|_{L_{\omega}^{q_{2}}\left(Q_{k, j}\right)} \\
&+\frac{\left[\omega\left(\widetilde{Q}_{k, j}\right)\right]^{1 / q_{2}}}{\left|\widetilde{Q}_{k, j}\right|}\|T(\lambda a)\|_{L_{\omega}^{q_{2}}\left(Q_{k, j}\right)}\left(\int_{\widetilde{Q}_{k, j}}[\omega(x)]^{-\frac{q_{2}^{\prime}}{q_{2}}} d x\right)^{1 / q_{2}{ }^{\prime}} \\
& \leq \widetilde{C}\|T(\lambda a)\|_{L_{\omega}^{q_{2}}\left(Q_{k, j}\right)},
\end{aligned}
$$

where $\widetilde{C}$ is a positive constant. Let

$$
\lambda_{k, i}:=\widetilde{C}\|T(\lambda a)\|_{L_{\omega}^{q_{2}}\left(Q_{k, j}\right)}\left[\omega\left(Q_{k, j}^{*}\right)\right]^{1-1 / q_{2}} \rho\left(\omega\left(Q_{k, j}^{*}\right)\right)
$$

and $b_{k, i}:=H_{k, i} / \lambda_{k, i}$. We then have $H_{k, i}=\lambda_{k, j} b_{k, j}$ and $b_{k, i}$ is a $\left(\rho, q_{2}, 0\right)$-atom. Moreover, by the assumption that $n q_{\omega}<(n+1) p_{\Phi}^{-}$and the definitions of $q_{\omega}$ and $p_{\Phi}^{-}$, we know that there exist $\widetilde{q} \in\left(q_{\omega}, \infty\right)$ and $p_{0} \in\left(0, p_{\Phi}^{-}\right)$such that $\Phi$ is of lower type $p_{0}$ and $n \widetilde{q}<(n+1) p_{0}$. Similar to the proof of (5.7), we know that

$$
\int_{R_{k}\left(Q_{0}\right)}|T(\lambda a)(x)|^{q_{2}} \omega(x) d x \lesssim 2^{-k(n+1) q_{2}} \frac{\|\lambda a\|_{L_{\omega}^{q_{2}}\left(Q_{0}\right)}^{q_{2}} \omega\left(R_{k}\left(Q_{0}\right)\right)}{\omega\left(Q_{0}\right)} .
$$

This, combined with Hölder's inequality, the lower type $p_{0}$ and the upper type 1 properties of $\Phi$, Lemma 2.2 (iii) and $n \widetilde{q}<(n+1) p_{0}$, implies that

$$
\begin{aligned}
& \sum_{k} \sum_{i} \omega\left(Q_{k, i}^{*}\right) \Phi\left(\frac{|\lambda|\|T(a)\|_{L_{\omega}^{q_{2}}\left(Q_{k, i}\right)}\left[\omega\left(Q_{k, i}^{*}\right)\right]^{1-1 / q_{2}} \rho\left(\omega\left(Q_{k, i}^{*}\right)\right)}{\left[\omega\left(Q_{k, i}^{*}\right)\right] \rho\left(\omega\left(Q_{k, i}^{*}\right)\right)}\right) \\
& \lesssim \sum_{k} \sum_{i} \omega\left(Q_{k, i}^{*}\right) \frac{\left[\omega\left(R_{k}\left(Q_{0}\right)\right)\right]^{1 / q_{2}}}{\left[\omega\left(Q_{k, i}\right)\right]^{1 / q_{2}}}\left[\frac{\|T(a)\|_{L_{\omega}^{q_{2}}\left(Q_{k, i}\right)}}{\|T(a)\|_{L_{\omega}^{q_{2}}\left(R_{k}\left(Q_{0}\right)\right)}}\right]^{p_{0}} \\
& \times \Phi\left(\frac{|\lambda|\|T(a)\|_{L_{\omega}^{q_{2}}\left(R_{k}\left(Q_{0}\right)\right)}}{\left[\omega\left(R_{k}\left(Q_{0}\right)\right)\right]^{1 / q_{2}}}\right) \\
& \lesssim \sum_{k}\left[\sum_{i} \omega\left(Q_{k, i}^{*}\right)\right]^{1 / q_{2}{ }^{\prime}} \frac{\left[\omega\left(R_{k}\left(Q_{0}\right)\right)\right]^{1 / q_{2}}}{\|T(a)\|_{L_{\omega}^{q_{2}}\left(R_{k}\left(Q_{0}\right)\right)}^{p_{0}}}\left[\sum_{i} \int_{Q_{k, i}}|T(a)(x)|^{q_{2}} \omega(x) d x\right]^{p_{0} / q_{2}} \\
& \times \Phi\left(\frac{2^{-k(n+1)}|\lambda|}{\omega\left(Q_{0}\right) \rho\left(\omega\left(Q_{0}\right)\right)}\right) \\
& \lesssim \sum_{k}\left[\int_{R_{k}\left(Q_{0}\right)} \omega(x) d x\right]^{1 / q_{2}^{\prime}} \frac{\left[\omega\left(R_{k}\left(Q_{0}\right)\right)\right]^{1 / q_{2}}}{\|T(a)\|_{L_{\omega}^{q_{2}}\left(R_{k}\left(Q_{0}\right)\right)}^{p_{0}}}
\end{aligned}
$$




$$
\begin{aligned}
& \times\|T(a)\|_{L_{\omega}^{q_{2}\left(R_{k}\left(Q_{0}\right)\right)}}^{p_{0}} \Phi\left(\frac{2^{-k(n+1)}|\lambda|}{\omega\left(Q_{0}\right) \rho\left(\omega\left(Q_{0}\right)\right)}\right) \\
\lesssim & \sum_{k} \omega\left(R_{k}\left(Q_{0}\right)\right) \Phi\left(\frac{2^{-k(n+1)}|\lambda|}{\omega\left(Q_{0}\right) \rho\left(\omega\left(Q_{0}\right)\right)}\right) \\
\lesssim & \sum_{k} 2^{-k\left[(n+1) p_{0}-n \widetilde{q}\right]} \omega\left(Q_{0}\right) \Phi\left(\frac{|\lambda|}{\omega\left(Q_{0}\right) \rho\left(\omega\left(Q_{0}\right)\right)}\right) \\
\lesssim & \omega\left(Q_{0}\right) \Phi\left(\frac{|\lambda|}{\omega\left(Q_{0}\right) \rho\left(\omega\left(Q_{0}\right)\right)}\right) .
\end{aligned}
$$

Therefore, we obtain $T(\lambda a) \in h_{\omega, r}^{\Phi}(\Omega)$ and

$$
\begin{aligned}
\|T(\lambda a)\|_{h_{\omega, r}^{\Phi}(\Omega)} \lesssim \inf \left\{s \in(0, \infty): \omega\left(Q_{0}^{*}\right) \Phi\left(\frac{|\lambda|}{s \omega\left(Q_{0}^{*}\right) \rho\left(\omega\left(Q_{0}^{*}\right)\right)}\right)\right. \\
\left.\quad+\sum_{k, i} \omega\left(Q_{k, i}^{*}\right) \Phi\left(\frac{\lambda_{k, i}}{s \omega\left(Q_{k, i}^{*}\right) \rho\left(\omega\left(Q_{k, i}^{*}\right)\right)}\right) \leq 1\right\} \\
\lesssim \inf \left\{s \in(0, \infty): \omega\left(Q_{0}\right) \Phi\left(\frac{|\lambda|}{s \omega\left(Q_{0}\right) \rho\left(\omega\left(Q_{0}\right)\right)}\right) \leq 1\right\} .
\end{aligned}
$$

From this and Theorem 1.7, we deduce that (5.14) and (5.15) hold in this case. This finishes the proof of Theorem 1.8 (ii) and hence Theorem 1.8

\section{Proof of Theorem 1.9}

In this section, we give the proof of Theorem 1.9. In what follows, we always assume that $\Omega$ is a bounded convex domain in $\mathbb{R}^{n}$. We first establish some auxiliary lemmas.

Let $L:=-\Delta$ with the Neumann boundary condition on $\Omega$. Denote by $\mathcal{D}(L)$ the domain of the operator $L$, and by $L^{k}$ the $k$-fold composition of $L$ with itself, in the sense of unbounded operators. In what follows, to simplify the notation, we just use $B^{\Omega}$ for $B\left(x_{B}, r_{B}\right) \cap \Omega$. For given $\lambda \in(0, \infty)$, we denote by $\lambda B^{\Omega}$ the set $B\left(x_{B}, \lambda r_{B}\right) \cap \Omega$. Let

$$
U_{0}^{\Omega}(B):=B^{\Omega} \text { and } U_{j}^{\Omega}(B):=\left(2^{j} B^{\Omega} \backslash 2^{j-1} B^{\Omega}\right) \text { for } j \in \mathbb{N} .
$$

Definition 6.1. Let $\Phi$ satisfy Assumption (A), $\omega \in A_{\infty}\left(\mathbb{R}^{n}\right)$ and $\Omega$ be an open subset of $\mathbb{R}^{n}$. Let $p_{\Phi}^{-}, \rho$ and $q_{\omega}$ be respectively as in (2.12), (2.14) and (2.5). Let

$$
M \in \mathbb{N} \text { and } M>\left\lfloor\frac{n}{2}\left(\frac{q_{\omega}}{p_{\Phi}^{-}}-1\right)\right\rfloor .
$$

A measurable function $a$ on $\Omega$ is called a local $(\rho, 2, M)_{\omega}$-atom if there exists a ball $B$ of $\mathbb{R}^{n}$ centered in $\Omega$ (but not necessarily included in $\Omega$ ) with radius $r_{B} \leq$ $2 \operatorname{diam}(\Omega)$ such that

$$
\|a\|_{L^{2}(\Omega)} \leq\left|B^{\Omega}\right|^{\frac{1}{2}}\left[\omega\left(B^{\Omega}\right) \rho\left(\omega\left(B^{\Omega}\right)\right)\right]^{-1}
$$

and either

(i) $r_{B}>1$, or 
(ii) $r_{B} \leq 1$ and there exists a function $b \in \mathcal{D}\left(L^{M}\right)$ such that $a=L^{M} b$, $\operatorname{supp}\left(L^{k} b\right) \subset B \cap \bar{\Omega}$ for all $k \in\{0,1, \cdots, M\}$, and

$$
\left\|\left(r_{B}^{2} L\right)^{k} b\right\|_{L^{2}(\Omega)} \leq r_{B}^{2 M}\left|B^{\Omega}\right|^{\frac{1}{2}}\left[\omega\left(B^{\Omega}\right) \rho\left(\omega\left(B^{\Omega}\right)\right)\right]^{-1}
$$

for all $k \in\{0,1, \cdots, M\}$.

Definition 6.2. Let $\Phi$ satisfy Assumption (A), $\omega \in A_{\infty}\left(\mathbb{R}^{n}\right), \Omega$ be a bounded, simply connected convex domain of $\mathbb{R}^{n}$ and $L=-\Delta$ with the Neumann boundary condition. Let $\rho$ and $M$ be respectively as in (2.14) and (6.1). A function $f \in L^{2}(\Omega)$ is said to be in $\widetilde{h}_{L, a t, M, \omega}^{\Phi}(\Omega)$ if there exist a sequence $\left\{a_{i}\right\}_{i}$ of $(\rho, 2, M)_{\omega}$-atoms and $\left\{\lambda_{i}\right\}_{i} \subset \mathbb{C}$ such that $f=\sum_{i} \lambda_{i} a_{i}$ in $L^{2}(\Omega)$ and

$$
\sum_{i} \omega\left(B_{i}^{\Omega}\right) \Phi\left(\frac{\left|\lambda_{i}\right|}{\omega\left(B_{i}^{\Omega}\right) \rho\left(\omega\left(B_{i}^{\Omega}\right)\right)}\right)<\infty,
$$

where for each $i, \operatorname{supp} a_{i} \subset B_{i}^{\Omega} \cap \bar{\Omega}$. Moreover, letting

$$
\Lambda\left(\left\{\lambda_{i} a_{i}\right\}_{i}\right):=\inf \left\{\lambda \in(0, \infty): \sum_{i} \omega\left(B_{i}^{\Omega}\right) \Phi\left(\frac{\left|\lambda_{i}\right|}{\lambda \omega\left(B_{i}^{\Omega}\right) \rho\left(\omega\left(B_{i}^{\Omega}\right)\right)}\right) \leq 1\right\},
$$

the quasi-norm of $f \in \widetilde{h}_{L, a t, M, \omega}^{\Phi}(\Omega)$ is defined by

$$
\|f\|_{h_{L, a t, M, \omega}^{\Phi}(\Omega)}:=\inf \left\{\Lambda\left(\left\{\lambda_{i} a_{i}\right\}_{i}\right)\right\},
$$

where the infimum is taken over all the decompositions of $f$ as above. The weighted local atomic Orlicz-Hardy space $h_{L, a t, M, \omega}^{\Phi}(\Omega)$ is defined to be the completion of $\widetilde{h}_{L, a t, M, \omega}^{\Phi}(\Omega)$ in the quasi-norm $\|\cdot\|_{h_{L, a t, M, \omega}^{\Phi}(\Omega)} \cdot$

Lemma 6.3. Let $\Phi, L, \Omega$ and $M$ be as in Definition 6.2 and $\omega$ as in Theorem 1.4. Then the spaces $h_{\omega, z}^{\Phi}(\Omega)$ and $h_{L, a t, M, \omega}^{\Phi}(\Omega)$ coincide with equivalent quasi-norms.

Proof. Similar to the proof of [26, Theorem 3.5], we have

$$
h_{\mathcal{N}_{h}, \omega}^{\Phi}(\Omega) \cap L^{2}(\Omega)=h_{L, a t, M, \omega}^{\Phi}(\Omega) \cap L^{2}(\Omega)
$$

with equivalent quasi-norms. From this, the following two facts that

$$
h_{\mathcal{N}_{h}, \omega}^{\Phi}(\Omega) \cap L^{2}(\Omega) \text { and } h_{L, a t, M, \omega}^{\Phi}(\Omega) \cap L^{2}(\Omega)
$$

are, respectively, dense in $h_{\mathcal{N}_{h}, \omega}^{\Phi}(\Omega)$ and $h_{L, a t, M, \omega}^{\Phi}(\Omega)$, and a density argument, we deduce that the spaces $h_{\mathcal{N}_{h}, \omega}^{\Phi}(\Omega)$ and $h_{L, a t, M, \omega}^{\Phi}(\Omega)$ coincide with equivalent quasinorms. By the assumption that $\Omega$ is a bounded convex domain of $\mathbb{R}^{n}$ and 26 , Lemma 2.8], we know that $\left(G_{1}\right)$ holds with $\mu=1$ for $L$. From this and Theorem 1.4(iii), we infer that the spaces $h_{\omega}^{\Phi}(\Omega)$ and $h_{L, a t, M, \omega}^{\Phi}(\Omega)$ coincide with equivalent quasi-norms. This finishes the proof of Lemma 6.3.

To show Theorem 1.9, we need the following useful estimates.

Lemma 6.4. Let $\Omega$ and $L$ be as in Definition 6.2. Denote by $\left\{K_{t}\right\}_{t \geq 0}$ the kernels of the semigroup $\left\{e^{-t L}\right\}_{t \geq 0}$. Let $q \in[1,2)$. Then there exist positive constants $\gamma$ and $C$ such that for all $y \in \Omega$ and $s, t \in(0, \infty)$,

$$
\left[\int_{\{x \in \Omega:|x-y| \geq \sqrt{s}\}}\left|\nabla_{x}^{2} K_{t}(x, y)\right|^{q} d x\right]^{\frac{1}{q}} \leq C t^{-1}\left|B^{\Omega}(y, \sqrt{t})\right|^{\frac{1}{q}-1} e^{-\gamma \frac{s}{t}} .
$$


Furthermore, for each $k \in \mathbb{N}$, there exist positive constants $\gamma_{k}$ and $C(k)$, depending on $k$, such that the $k$-th order time derivative $\frac{d^{k}}{d t^{k}} K_{t}$ of the kernel $K_{t}$ satisfies that for all $y \in \Omega$ and $s, t \in(0, \infty)$,

$$
\begin{aligned}
& {\left[\int_{\{x \in \Omega:|x-y| \geq \sqrt{s}\}}\left|\nabla_{x}^{2}\left(\frac{d^{k}}{d t^{k}} K_{t}(x, y)\right)\right|^{q} d x\right]^{\frac{1}{q}}} \\
& \quad \leq C(k) t^{-(k+1)}\left|B^{\Omega}(y, \sqrt{t})\right|^{\frac{1}{q}-1} e^{-\gamma_{k} \frac{s}{t}} .
\end{aligned}
$$

Proof. We first prove (6.2). It was shown in [26, Proposition 4.15] that for some $\gamma_{1} \in(0, \infty)$, there exists a positive constant $C\left(\gamma_{1}\right)$ such that for all $y \in \Omega$ and $t \in(0, \infty)$,

$$
\int_{\Omega}\left|\nabla_{x}^{2} K_{t}(x, y)\right|^{2} e^{\frac{\gamma_{1}|x-y|^{2}}{t}} d x \leq C\left(\gamma_{1}\right) t^{-2}|B(y, \sqrt{t})|^{-1} .
$$

Moreover, it was obtained in [26. Lemma 4.13] that for any $\gamma_{2} \in(0, \infty)$, there exists a positive constant $C\left(\gamma_{2}\right)$ such that for all $s \in[0, \infty), t \in(0, \infty)$ and $y \in \Omega$,

$$
\int_{\{x \in \Omega:|x-y| \geq \sqrt{s}\}} e^{-2 \gamma_{2} \frac{|x-y|^{2}}{t}} d x \leq C\left(\gamma_{2}\right)\left|B^{\Omega}(y, \sqrt{t})\right| e^{-\gamma_{2} \frac{s}{t}} .
$$

This, together with Hölder's inequality and (6.4), implies that

$$
\begin{aligned}
& {\left[\int_{\{x \in \Omega:|x-y| \geq \sqrt{s}\}}\left|\nabla_{x}^{2} K_{t}(x, y)\right|^{q} d x\right]^{\frac{1}{q}}} \\
& \quad \leq\left[\int_{\Omega}\left|\nabla_{x}^{2} K_{t}(x, y)\right|^{2} e^{\frac{\gamma_{1}|x-y|^{2}}{t}} d x\right]^{\frac{1}{2}}\left[\int_{\{x \in \Omega:|x-y| \geq \sqrt{s}\}} e^{-\frac{\gamma_{1} q}{2-q} \frac{|x-y|^{2}}{t}} d x\right]^{\frac{1}{q}-\frac{1}{2}} \\
& \lesssim t^{-1}\left|B^{\Omega}(y, \sqrt{t})\right|^{-\frac{1}{2}} e^{-\gamma s / t}\left|B^{\Omega}(y, \sqrt{t})\right|^{\frac{1}{q}-\frac{1}{2}} \lesssim t^{-1} e^{-\gamma s / t}\left|B^{\Omega}(y, \sqrt{t})\right|^{\frac{1}{q}-1}
\end{aligned}
$$

where $\gamma:=\gamma_{1} / 4$, which implies that (6.2) holds.

The proof of (6.3) is similar to that of [26, Lemma 4.12]. We omit the details. This finishes the proof of Lemma 6.4

The following Lemma 6.5] is just [26, Theorem 4.2].

Lemma 6.5. Let $\Omega$ be a bounded, simply connected semiconvex domain in $\mathbb{R}^{n}$ and $\mathbb{G}_{N}$ the Neumann Green operator for the problem (1.2). Then the operators in (1.4), originally defined on $C^{\infty}(\bar{\Omega})$, can be extended to bounded operators on $L^{p}(\Omega)$ for $p \in(1,2]$.

Now we prove Theorem 1.9 by using Lemmas 6.3 and 6.4 .

Proof of Theorem 1.9. We borrow some ideas from [26. Fix $m, s \in\{1, \cdots, n\}$ and denote by $T$ the operator $\frac{\partial^{2} \mathbb{G}_{N}}{\partial x_{m} \partial x_{s}}$. We first prove Theorem 1.9)(i). Let $f \in$ $h_{\omega, z}^{\Phi}(\Omega) \cap L^{2}(\Omega)$ and $M$ be as in (6.1) satisfying $M>\frac{n q_{\omega}}{2 p_{\Phi}^{-}}$. Then by Lemma 6.3, we know that there exist a sequence $\left\{a_{k}\right\}_{k}$ of $(\rho, 2, M)_{\omega}$-atoms and a sequence $\left\{\lambda_{k}\right\}_{k}$ of numbers such that

$$
f=\sum_{k} \lambda_{k} a_{k}
$$


and

$$
\|f\|_{h_{\omega, z}^{\Phi}(\Omega)} \sim \Lambda\left(\left\{\lambda_{k} a_{k}\right\}_{k}\right),
$$

where $\Lambda\left(\left\{\lambda_{k} a_{k}\right\}_{k}\right)$ is as in Definition 6.2,

To finish the proof of Theorem 1.9(i), we need to show that for any $(\rho, 2, M)_{\omega^{-}}$ atom $a$ supported in the ball $B\left(x_{0}, r_{0}\right) \cap \bar{\Omega}$ and any $\lambda \in \mathbb{C}$,

$$
\int_{\Omega} \Phi(T(\lambda a)(x)) \omega(x) d x \lesssim \omega\left(B_{0}^{\Omega}\right) \Phi\left(\frac{|\lambda|}{\omega\left(B_{0}^{\Omega}\right) \rho\left(\omega\left(B_{0}^{\Omega}\right)\right)}\right),
$$

where and in what follows, $B_{0}^{\Omega}:=B\left(x_{0}, r_{0}\right) \cap \Omega$.

Indeed, if (6.7) holds, then by (6.5) and the assumption that $\Phi$ is subadditive, we know that for all $\lambda \in(0, \infty)$,

$$
\begin{aligned}
\int_{\Omega} \Phi\left(T\left(\frac{f}{\lambda}\right)(x)\right) \omega(x) d x & \leq \sum_{k} \int_{\Omega} \Phi\left(T\left(\frac{\lambda_{k} a_{k}}{\lambda}\right)(x)\right) \omega(x) d x \\
& \lesssim \sum_{k} \omega\left(B_{k}^{\Omega}\right) \Phi\left(\frac{\left|\lambda_{k}\right|}{\lambda \omega\left(B_{k}^{\Omega}\right) \rho\left(\omega\left(B_{k}^{\Omega}\right)\right)}\right)
\end{aligned}
$$

where for each $k, B_{k}^{\Omega}:=B\left(x_{k}, r_{k}\right) \cap \Omega$ and supp $a_{k} \subset B\left(x_{k}, r_{k}\right) \cap \bar{\Omega}$, which, together with (6.6), implies that

$$
\|T(f)\|_{L_{\omega}^{\Phi}(\Omega)} \lesssim\|f\|_{h_{\omega, z}^{\Phi}(\Omega)} .
$$

From this, the fact that $h_{\omega, z}^{\Phi}(\Omega) \cap L^{2}(\Omega)$ is dense in $h_{\omega, z}^{\Phi}(\Omega)$ and a density argument, we deduce that Theorem 1.9)(i) holds.

Now we prove (6.7) by considering the following two cases for $r_{0}$.

Case i) $r_{0} \geq 1$. In this case, the proof of (6.7) is similar to the proof of (5.4). We omit the details.

Case ii) $r_{0}<1$. In this case, we have

$$
\int_{\Omega} \Phi(T(\lambda a)(x)) \omega(x) d x=\sum_{j=0}^{\infty} \int_{U_{j}^{\Omega}\left(B_{0}\right)} \Phi(T(\lambda a)(x)) \omega(x) d x=: \sum_{j=0}^{\infty} \mathrm{I}_{j} .
$$

For $j \in\{0,1,2\}$, similar to the estimate of (5.6), we know that

$$
\mathrm{I}_{j} \lesssim \omega\left(B_{0}^{\Omega}\right) \Phi\left(\frac{|\lambda|}{\omega\left(B_{0}^{\Omega}\right) \rho\left(\omega\left(B_{0}^{\Omega}\right)\right)}\right) .
$$

Now we deal with $\mathrm{I}_{j}$ for $j \in \mathbb{N}$ with $j \geq 3$. Take $q \in(1,2)$ such that $\omega \in R H_{q^{\prime}}\left(\mathbb{R}^{n}\right)$, where $\frac{1}{q}+\frac{1}{q^{\prime}}=1$. Then from Jensen's inequality, Hölder's inequality, and Lemma 2.2(v), we deduce that

$$
\begin{aligned}
\mathrm{I}_{j} \leq & \omega\left(U_{j}^{\Omega}\left(B_{0}\right)\right) \Phi\left(\frac{1}{\omega\left(U_{j}^{\Omega}\left(B_{0}\right)\right)}\left\{\int_{U_{j}^{\Omega}\left(B_{0}\right)}|T(\lambda a)(x)|^{q} d x\right\}^{\frac{1}{q}}\right. \\
& \left.\times\left\{\int_{U_{j}^{\Omega}\left(B_{0}\right)}[\omega(x)]^{q^{\prime}} d x\right\}^{\frac{1}{q^{\prime}}}\right) \\
\lesssim & \omega\left(U_{j}^{\Omega}\left(B_{0}\right)\right) \Phi\left(\frac{1}{\left|U_{j}^{\Omega}\left(B_{0}\right)\right|^{\frac{1}{q}}}\left\{\int_{U_{j}^{\Omega}\left(B_{0}\right)}|T(\lambda a)(x)|^{q} d x\right\}^{\frac{1}{q}}\right) .
\end{aligned}
$$


Moreover, since

$$
T(\lambda a)=2 \int_{0}^{\infty} \frac{\partial^{2} e^{-2 t L}(\lambda a)}{\partial x_{m} \partial x_{s}} d t
$$

we conclude that for each $j \in \mathbb{N}$ with $j \geq 3$,

(6.10) $\|T(\lambda a)\|_{L^{q}\left(U_{j}^{\Omega}\left(B_{0}\right)\right)}$

$$
\begin{aligned}
& \leq 2\left\|\int_{0}^{r_{0}^{2}} \frac{\partial^{2} e^{-2 t L}(\lambda a)}{\partial x_{m} \partial x_{s}} d t\right\|_{L^{q}\left(U_{j}^{\Omega}\left(B_{0}\right)\right)}+2\left\|\int_{r_{0}^{2}}^{[\operatorname{diam}(\Omega)]^{2}} \cdots d t\right\|_{L^{q}\left(U_{j}^{\Omega}\left(B_{0}\right)\right)} \\
& \quad+2\left\|\int_{[\operatorname{diam}(\Omega)]^{2}}^{\infty} \cdots d t\right\|_{L_{\left(U_{j}^{\Omega}\left(B_{0}\right)\right)}}=: \mathrm{I}+\mathrm{II}+\mathrm{III} .
\end{aligned}
$$

We first estimate I. By $j \geq 3$, we know that $\operatorname{dist}\left(U_{j}^{\Omega}\left(B_{0}\right), B_{0}^{\Omega}\right) \geq 2^{j-2} r_{0}$. From this and Minkowski's inequality, Lemma 3.9 and (6.2), we infer that

$$
\begin{aligned}
\left\|\frac{\partial^{2} e^{-2 t L}(\lambda a)}{\partial x_{m} \partial x_{s}}\right\|_{L^{q}\left(U_{j}^{\Omega}\left(B_{0}\right)\right)} & \leq \int_{B_{0}^{\Omega}}\left[\int_{U_{j}^{\Omega}\left(B_{0}\right)}\left|\nabla_{x}^{2} K_{t}(x, y)\right|^{q} d x\right]^{\frac{1}{q}}|\lambda a(y)| d y \\
& \lesssim|\lambda|\|a\|_{L^{1}(\Omega)} t^{\frac{n}{2}\left(\frac{1}{q}-1\right)-1} e^{-\gamma \frac{\left[^{j} r_{0}\right]^{2}}{t}}
\end{aligned}
$$

which, together with Minkowski's inequality, implies that, for any $M_{0} \in\left(\frac{n}{2}(1-\right.$ $\left.\left.\frac{1}{q}\right), \infty\right)$,

$$
\begin{aligned}
\mathrm{I} & \lesssim|\lambda|\|a\|_{L^{1}(\Omega)} \int_{0}^{r_{0}^{2}} e^{-\gamma \frac{\left[2^{j} r_{0}\right]^{2}}{t}} t^{\frac{n}{2}\left(\frac{1}{q}-1\right)-1} d t \\
& \lesssim|\lambda|\|a\|_{L^{2}(\Omega)}\left|B_{0}^{\Omega}\right|^{\frac{1}{2}} \int_{0}^{r_{0}^{2}}\left(\frac{t}{\left[2^{j} r_{0}\right]^{2}}\right)^{M_{0}} t^{\frac{n}{2}\left(\frac{1}{q}-1\right)-1} d t \\
& \lesssim 2^{-2 j M_{0}} \frac{|\lambda|\left|B_{0}\right|^{\frac{1}{q}}}{\omega\left(B_{0}^{\Omega}\right) \rho\left(\omega\left(B_{0}^{\Omega}\right)\right)} .
\end{aligned}
$$

Now we deal with II. Pick $M_{1} \in\left(\frac{n q_{\omega}}{2 p_{\Phi}^{-}}, M\right)$. By $a=L^{M} b$, the fact that for each $k \in \mathbb{N}$,

$$
(-1)^{k} L^{k} e^{-t L}=\frac{d^{k}}{d t^{k}} e^{-t L}
$$

Lemma 3.9 and (6.3), we conclude that

$$
\begin{aligned}
\mathrm{II} & \lesssim|\lambda| \int_{r_{0}^{2}}^{[\operatorname{diam}(\Omega)]^{2}}\left\|\frac{\partial^{2}}{\partial x_{m} \partial x_{s}}\left(\frac{d^{M}}{d t^{M}} e^{-2 t L} b\right)\right\|_{L^{q}\left(U_{j}^{\Omega}\left(B_{0}\right)\right)} d t \\
& \lesssim|\lambda| \int_{r_{0}^{2}}^{[\operatorname{diam}(\Omega)]^{2}} \int_{B_{0}^{\Omega}}\left[\int_{U_{j}^{\Omega}\left(B_{0}\right)}\left|\nabla_{x}^{2}\left(\frac{d^{M}}{d t^{M}} K_{t}\right)(x, y)\right|^{q} d x\right]^{\frac{1}{q}}|b(y)| d y d t \\
& \lesssim|\lambda|\|b\|_{L^{1}(\Omega)} \int_{r_{0}^{2}}^{[\operatorname{diam}(\Omega)]^{2}} e^{-\gamma^{\frac{\left[2^{j} r_{0}\right]^{2}}{t}} t^{\frac{n}{2}\left(\frac{1}{q}-1\right)-(M+1)} d t} \\
& \lesssim|\lambda|\|b\|_{L^{2}(\Omega)}\left|B_{0}^{\Omega}\right|^{\frac{1}{2}} \int_{r_{0}^{2}}^{[\operatorname{diam}(\Omega)]^{2}}\left(\frac{t}{\left[2^{j} r_{0}\right]^{2}}\right)^{M_{1}} t^{\frac{n}{2}\left(\frac{1}{q}-1\right)-(M+1)} d t
\end{aligned}
$$




$$
\lesssim 2^{-2 j M_{1}} \frac{|\lambda|\left|B_{0}\right|^{\frac{1}{q}}}{\omega\left(B_{0}^{\Omega}\right) \rho\left(\omega\left(B_{0}^{\Omega}\right)\right)} .
$$

For III, similar to (6.12), we have

$$
\begin{aligned}
\mathrm{III} & \lesssim|\lambda| \int_{[\operatorname{diam}(\Omega)]^{2}}^{\infty}\left\|\frac{\partial^{2}}{\partial x_{m} \partial x_{s}}\left(\frac{d^{M}}{d t^{M}} e^{-2 t L} b\right)\right\|_{L^{q}\left(U_{j}^{\Omega}\left(B_{0}\right)\right)} d t \\
& \lesssim|\lambda| \int_{[\operatorname{diam}(\Omega)]^{2}}^{\infty} \int_{B_{0}^{\Omega}}\left[\int_{U_{j}^{\Omega}\left(B_{0}\right)}\left|\nabla_{x}^{2}\left(\frac{d^{M}}{d t^{M}} K_{t}\right)(x, y)\right|^{q} d x\right]^{\frac{1}{q}}|b(y)| d y d t \\
& \lesssim|\lambda|\|b\|_{L^{1}(\Omega)} \int_{[\operatorname{diam}(\Omega)]^{2}}^{\infty} e^{-\gamma \frac{\left[2^{j} r_{0}\right]^{2}}{t}} t^{-(M+1)} d t \\
& \lesssim|\lambda|\|b\|_{L^{2}(\Omega)} \mid B_{0}^{\Omega||^{\frac{1}{2}}} \int_{[\operatorname{diam}(\Omega)]^{2}}^{\infty}\left(\frac{t}{\left[2^{j} r_{0}\right]^{2}}\right)^{M_{1}} t^{-(M+1)} d t \\
& \lesssim 2^{-2 j M_{1}} \frac{|\lambda|\left|B_{0}\right| r_{0}^{2\left(M-M_{1}\right)}}{\omega\left(B_{0}^{\Omega}\right) \rho\left(\omega\left(B_{0}^{\Omega}\right)\right)} \lesssim 2^{-2 j M_{1}} \frac{|\lambda|\left|B_{0}\right|^{\frac{1}{q}}}{\omega\left(B_{0}^{\Omega}\right) \rho\left(\omega\left(B_{0}^{\Omega}\right)\right)}
\end{aligned}
$$

where we used the assumption that $\Omega$ is bounded and $B(y, \sqrt{t}) \cap \Omega=\Omega$ for all $t \in\left([\operatorname{diam}(\Omega)]^{2}, \infty\right)$ in the third inequality, and the assumption that $r_{B}<1$ in the last inequality.

By $M_{0}>\frac{n q_{\omega}}{2 p_{\Phi}}$, we know that there exist $p_{0} \in\left(0, p_{\Phi}^{-}\right)$and $q_{0} \in\left(q_{\omega}, \infty\right)$ such that $\Phi$ is of lower type $p_{0}$ and $M_{0}>\frac{n q_{0}}{2 p_{0}}$. Thus, from (6.9), (6.10), (6.11), (6.12), (6.13), Lemma $2.2\left(\right.$ (iii), the lower type $p_{0}$ property of $\Phi$ and $M_{0}>\frac{n q_{0}}{2 p_{0}}$, we deduce that

$$
\begin{aligned}
\sum_{j=3}^{\infty} \mathrm{I}_{j} & \lesssim \sum_{j=3}^{\infty} \omega\left(U_{j}^{\Omega}\left(B_{0}\right)\right) \Phi\left(\frac{2^{-2 j M_{1}}|\lambda|}{\mid U_{j}^{\Omega}\left(B_{0}\right)^{\frac{1}{q}}} \frac{\left|B_{0}\right|^{\frac{1}{q}}}{\omega\left(B_{0}^{\Omega}\right) \rho\left(\omega\left(B_{0}^{\Omega}\right)\right)}\right) \\
& \lesssim \sum_{j=3}^{\infty} 2^{j n q_{0}} 2^{-\left(2 M_{1}+n / q\right) j p_{0}} \omega\left(B_{0}^{\Omega}\right) \Phi\left(\frac{|\lambda|}{\omega\left(B_{0}^{\Omega}\right) \rho\left(\omega\left(B_{0}^{\Omega}\right)\right)}\right) \\
& \lesssim \omega\left(B_{0}^{\Omega}\right) \Phi\left(\frac{|\lambda|}{\omega\left(B_{0}^{\Omega}\right) \rho\left(\omega\left(B_{0}^{\Omega}\right)\right)}\right)
\end{aligned}
$$

which, together with (6.8), implies that (6.7) holds in this case. This finishes the proof of Theorem 1.9(i).

Now we prove Theorem 1.9(ii). For each $y \in \Omega$, let $V(\cdot, y)$ be the solution of the Neumann problem

$$
\begin{cases}\Delta V(\cdot, y)=|\Omega|^{-1}, & \text { in } \Omega, \\ \partial_{\nu(x)}[V(x, y)]=\partial_{\nu(x)}[\Gamma(x-y)], & \text { for } x \in \partial \Omega\end{cases}
$$

where $\nu(x)$ denotes the outward unit normal to $\partial \Omega$ at $x \in \partial \Omega$. Then a convenient way of expressing the Green function $G_{N}$ for $L=-\Delta$ with the Neumann boundary condition on $\Omega$ (namely, the integral kernel of the Neumann Green operator $\mathbb{G}_{N}$ ) is

$$
G_{N}(x, y)=\Gamma(x-y)-V(x, y), \quad x, y \in \Omega, x \neq y .
$$


The Neumann problem (1.3) has a unique solution, up to an additive constant, given by

$$
\begin{aligned}
\mathbb{G}_{N}(f)(x) & =\int_{\Omega} G_{N}(x, y) f(y) d y \\
& =\int_{\Omega} \Gamma(x-y) f(y) d y-\int_{\Omega} V(x, y) f(y) d y \\
& =: E(f)(x)-V(f)(x),
\end{aligned}
$$

where $f \in C^{\infty}(\Omega)$ satisfies that

$$
\int_{\Omega} f(y) d y=0
$$

Let $f \in h_{\omega, z}^{\Phi}(\Omega) \cap L^{2}(\Omega)$. Then by Lemma 6.3, we know that there exist a sequence $\left\{a_{k}\right\}_{k}$ of $(\rho, 2, M)_{\omega}$-atoms and a sequence $\left\{\lambda_{k}\right\}_{k}$ of numbers such that (6.5) and (6.6) hold.

To finish the proof of Theorem 1.9(ii), we only need to show that for any $(\rho, 2, M)_{\omega}$-atom $a$ supported in the ball $B\left(x_{0}, r_{0}\right) \cap \bar{\Omega}$ and any $\lambda \in \mathbb{C}$,

$$
\int_{\Omega} \Phi\left([T(\lambda a)]_{\Omega, \varphi}^{+}(x)\right) \omega(x) d x \lesssim \omega\left(B_{0}^{\Omega}\right) \Phi\left(\frac{|\lambda|}{\omega\left(B_{0}^{\Omega}\right) \rho\left(\omega\left(B_{0}^{\Omega}\right)\right)}\right)
$$

where and in what follows, $B_{0}^{\Omega}:=B\left(x_{0}, r_{0}\right) \cap \Omega$, and $[T(\lambda a)]_{\Omega, \varphi}^{+}$is as in (1.7).

Indeed, if (6.16) holds, then by (6.5) and the assumption that $\Phi$ is subadditive, we conclude that for all $\lambda \in(0, \infty)$,

$$
\begin{aligned}
\int_{\Omega} \Phi\left(\left[T\left(\frac{f}{\lambda}\right)\right]_{\Omega, \varphi}^{+}(x)\right) \omega(x) d x & \leq \sum_{k} \int_{\Omega} \Phi\left(\left[T\left(\frac{\lambda_{k} a_{k}}{\lambda}\right)\right]_{\Omega, \varphi}^{+}(x)\right) \omega(x) d x \\
& \lesssim \sum_{k} \omega\left(B_{k}^{\Omega}\right) \Phi\left(\frac{\left|\lambda_{k}\right|}{\lambda \omega\left(B_{k}^{\Omega}\right) \rho\left(\omega\left(B_{k}^{\Omega}\right)\right)}\right)
\end{aligned}
$$

where for each $k, B_{k}^{\Omega}:=B\left(x_{k}, r_{k}\right) \cap \Omega$ and supp $a_{k} \subset B\left(x_{k}, r_{k}\right) \cap \bar{\Omega}$, which, together with Theorem 1.7 and (6.6), implies that

$$
\|T(f)\|_{h_{\omega, r}^{\Phi}(\Omega)} \lesssim\|f\|_{h_{\omega, z}^{\Phi}(\Omega)} .
$$

From this, the fact that $h_{\omega, z}^{\Phi}(\Omega) \cap L^{2}(\Omega)$ is dense in $h_{\omega, z}^{\Phi}(\Omega)$ and a density argument, we deduce that Theorem [1.9)(ii) holds.

Now we prove (6.16). By (6.15), it suffices to show

$$
\begin{aligned}
& \int_{\Omega} \Phi\left(\left[\frac{\partial^{2} E(\lambda a)}{\partial x_{m} \partial x_{s}}\right]_{\Omega, \varphi}^{+}(x)\right) \omega(x) d x+\int_{\Omega} \Phi\left(\left[\frac{\partial^{2} V(\lambda a)}{\partial x_{m} \partial x_{s}}\right]_{\Omega, \varphi}^{+}(x)\right) \omega(x) d x \\
& \quad \lesssim \omega\left(B_{0}^{\Omega}\right) \Phi\left(\frac{|\lambda|}{\omega\left(B_{0}^{\Omega}\right) \rho\left(\omega\left(B_{0}^{\Omega}\right)\right)}\right) .
\end{aligned}
$$


By the fact that $L$ conserves probability, namely, $e^{-t L} 1=1$ for all $t \in(0, \infty)$, we know that

$$
\int_{\Omega} a(x) d x=0
$$

(see also [26, (5.4)]). Then, similar to the proof of [86, Theorem 8.2], we have

$$
\int_{\Omega} \Phi\left(\left[\frac{\partial^{2} E(\lambda a)}{\partial x_{m} \partial x_{s}}\right]_{\Omega, \varphi}^{+}(x)\right) \omega(x) d x \sim\left\|\frac{\partial^{2} E(\lambda a)}{\partial x_{m} \partial x_{s}}\right\|_{h_{\omega, r}^{\Phi}(\Omega)} \lesssim\|\lambda a\|_{h_{\omega, r}^{\Phi}(\Omega)} .
$$

Moreover, by (6.14) and (6.15), we see that $\frac{\partial^{2} V(\lambda a)}{\partial x_{m} \partial x_{s}}$ is harmonic in $\Omega$. Hence, an application of Theorem 1.7. in which we take the function $\varphi$ to be radial, yields, on account of the mean value property for harmonic functions, that for all $x \in \Omega$,

$$
\left(\frac{\partial^{2} V(\lambda a)}{\partial x_{m} \partial x_{s}}\right)_{\Omega, \varphi}^{+}(x)=\left|\frac{\partial^{2} V(\lambda a)}{\partial x_{m} \partial x_{s}}(x)\right|,
$$

which, together with Theorems 1.7 and 1.9(i), and Lemma 6.3 implies that

$$
\begin{aligned}
\left\|\frac{\partial^{2} V(\lambda a)}{\partial x_{m} \partial x_{s}}\right\|_{h_{\omega, r}^{\Phi}(\Omega)} & \sim\left\|\left(\frac{\partial^{2} V(\lambda a)}{\partial x_{m} \partial x_{s}}\right)_{\Omega, \varphi}^{+}\right\|_{L_{\omega}^{\Phi}(\Omega)} \sim\left\|\frac{\partial^{2} V(\lambda a)}{\partial x_{m} \partial x_{s}}\right\|_{L_{\omega}^{\Phi}(\Omega)} \\
& \lesssim\left\|\frac{\partial^{2} E(\lambda a)}{\partial x_{m} \partial x_{s}}\right\|_{L_{\omega}^{\Phi}(\Omega)}+\left\|\frac{\partial^{2} \mathbb{G}_{N}(\lambda a)}{\partial x_{m} \partial x_{s}}\right\|_{L_{\omega}^{\Phi}(\Omega)} \lesssim\|\lambda a\|_{h_{\omega, r}^{\Phi}(\Omega)} .
\end{aligned}
$$

This, combined with (6.17) and Lemma 6.3 implies that (6.16) holds, which completes the proof of Theorem 1.9.

\section{ACKNOWLEDGEMENTS}

The authors would like to thank Professor Lixin Yan for some suggestive discussions on the topic of this paper.

\section{REFERENCES}

[1] V. Adolfsson, $L^{2}$-integrability of second-order derivatives for Poisson's equation in nonsmooth domains, Math. Scand. 70 (1992), 146-160. MR1174208 (93h:35047)

[2] V. Adolfsson, $L^{p}$-integrability of the second order derivatives of Green potentials in convex domains, Pacific J. Math. 159 (1993), 201-225. MR1214070(94c:35041)

[3] V. Adolfsson and D. Jerison, $L^{p}$-integrability of the second order derivatives for the Neumann problem in convex domains, Indiana Univ. Math. J. 43 (1994), 1123-1138. MR.1322613 (96e:35031)

[4] S. Agmon, A. Douglis and L. Nirenberg, Estimates near the boundary for solutions of elliptic partial differential equations satisfying general boundary conditions. I, Comm. Pure Appl. Math. 12 (1959), 623-727. MR0125307 (23:A2610)

[5] T. Aoki, Locally bounded linear topological space, Proc. Imp. Acad. Tokyo 18 (1942), 588-594. MR0014182 (7:250d)

[6] P. Auscher, X. T. Duong and A. McIntosh, Boundedness of Banach space valued singular integral operators and Hardy spaces, Unpublished Manuscript, 2005.

[7] P. Auscher, A. McIntosh and E. Russ, Hardy spaces of differential forms on Riemannian manifolds, J. Geom. Anal. 18 (2008), 192-248. MR2365673 (2009d:42053)

[8] P. Auscher and E. Russ, Hardy spaces and divergence operators on strongly Lipschitz domains of $\mathbb{R}^{n}$, J. Funct. Anal. 201 (2003), 148-184. MR1986158 (2004c:42049) 
[9] P. Auscher and Ph. Tchamitchian, Square root problem for divergence operators and related topics, Astérisque 249 (1998), viii+172 pp. MR1651262 (2000c:47092)

[10] P. Auscher and Ph. Tchamitchian, Gaussian estimates for second order elliptic divergence operators on Lipschitz and $C^{1}$ domains, Evolution equations and their applications in physical and life sciences (Bad Herrenalb, 1998), pp. 15-32, Lecture Notes in Pure and Applied Math., 215, Dekker, New York, 2001. MR.1816433 (2001m:35074)

[11] Z. Birnbaum and W. Orlicz, Über die verallgemeinerung des begriffes der zueinander konjugierten potenzen, Studia Math. 3 (1931), 1-67.

[12] H. Bui, Weighted Hardy spaces, Math. Nachr. 103 (1981), 45-62. MR653914 (83h:42026)

[13] S.-S. Byun, F. Yao and S. Zhou, Gradient estimates in Orlicz space for nonlinear elliptic equations, J. Funct. Anal. 255 (2008), 1851-1873. MR2462578(2010b:35153)

[14] A. Carbonaro, A. McIntosh and A. J. Morris, Local Hardy spaces of differential forms on Riemannian manifolds, J. Geom. Anal. 23 (2013), 106-169.

[15] D.-C. Chang, The dual of Hardy spaces on a bounded domain in $\mathbb{R}^{n}$, Forum Math. 6 (1994), 65-81. MR1253178 (95b:42022)

[16] D.-C. Chang, G. Dafni and E. M. Stein, Hardy spaces, BMO and boundary value problems for the Laplacian on a smooth domain in $\mathbb{R}^{n}$, Trans. Amer. Math. Soc. 351 (1999), 1605-1661. MR.1458319 (99f:46031)

[17] D.-C. Chang, S. G. Krantz and E. M. Stein, Hardy spaces and elliptic boundary value problems, Contemporary Math. 137 (1992), 119-131. MR.1190976

[18] D.-C. Chang, S. G. Krantz and E. M. Stein, $H^{p}$ theory on a smooth domain in $\mathbb{R}^{N}$ and elliptic boundary value problems, J. Funct. Anal. 114 (1993), 286-347. MR1223705 (94j:46032)

[19] R. R. Coifman, P.-L. Lions, Y. Meyer and S. Semmes, Compensated compactness and Hardy spaces, J. Math. Pures Appl. (9) 72 (1993), 247-286. MR.1225511 (95d:46033)

[20] R. R. Coifman, Y. Meyer and E. M. Stein, Some new function spaces and their applications to harmonic analysis, J. Funct. Anal. 62 (1985), 304-335. MR791851 (86i:46029)

[21] R. R. Coifman and G. Weiss, Analyse Harmonique Non-commutative sur Certains Espaces Homogènes, Lecture Notes in Math., 242, Springer, Berlin, 1971. MR0499948 (58:17690)

[22] R. R. Coifman and G. Weiss, Extensions of Hardy spaces and their use in analysis, Bull. Amer. Math. Soc. 83 (1977), 569-645. MR0447954 (56:6264)

[23] D. Cruz-Uribe and C. J. Neugebauer, The structure of the reverse Hölder classes, Trans. Amer. Math. Soc. 347 (1995), 2941-2960. MR1308005 (95m:42026)

[24] B. Dahlberg, $L^{q}$-estimates for Green potentials in Lipschitz domains, Math. Scand. 44 (1979), 149-170. MR544584 (81d:31007)

[25] B. Dahlberg, G. Verchota and T. Wolff, Unpublished manuscript.

[26] X. T. Duong, S. Hofmann, D. Mitrea, M. Mitrea and L. Yan, Hardy spaces and regularity for the inhomogeneous Dirichlet and Neumann problems, Rev. Mat. Iberoam. 29 (2013), $183-236$.

[27] X. T. Duong and L. Yan, On the atomic decomposition for Hardy spaces on Lipschitz domains of $\mathbb{R}^{n}$, J. Funct. Anal. 215 (2004), 476-486. MR2151301 (2006d:42040)

[28] X. T. Duong and L. Yan, Duality of Hardy and BMO spaces associated with operators with heat kernel bounds, J. Amer. Math. Soc. 18 (2005), 943-973. MR2163867 (2006d:42037)

[29] X. T. Duong, J. Xiao and L. Yan, Old and new Morrey spaces with heat kernel bounds, J. Fourier Anal. Appl. 13 (2007), 87-111. MR2296729 (2007k:42027)

[30] C. Fefferman and E. M. Stein, $H^{p}$ spaces of several variables, Acta Math. 129 (1972), 137193. MR0447953(56:6263)

[31] S. Fromm, Potential space estimates for Green potentials in convex domains, Proc. Amer. Math. Soc. 119 (1993), 225-233. MR1156467 (93k:35076)

[32] J. García-Cuerva, Weighted $H^{p}$ spaces, Dissertationes Math. (Rozprawy Mat.) 162 (1979), 1-63. MR549091 (82a:42018)

[33] J. García-Cuerva and J. Rubio de Francia, Weighted Norm Inequalities and Related Topics, Amsterdam, North-Holland, 1985. MR807149 (87d:42023)

[34] L. Grafakos, Modern Fourier Analysis, Second Edition, Graduate Texts in Math., No. 250, Springer, New York, 2008. MR2463316 (2011d:42001)

[35] P. Grisvard, Elliptic Problems in Nonsmooth Domains, Monographs and Studies in Mathematics, 24, Pitman (Advanced Publishing Program), Boston, MA, 1985. MR775683 (86m:35044) 
[36] M. Grüter and K.-O. Widman, The Green function for uniformly elliptic equations, Manuscripta Math. 37 (1982), 303-342. MR657523 (83h:35033)

[37] D. Goldberg, A local version of real Hardy spaces, Duke Math. J. 46 (1979), 27-42. MR.523600 (80h:46052)

[38] S. Hofmann, G. Lu, D. Mitrea, M. Mitrea and L. Yan, Hardy spaces associated to nonnegative self-adjoint operators satisfying Davies-Gaffney estimates, Mem. Amer. Math. Soc. 214 (2011), no. 1007, vi+78 pp. MR.2868142

[39] S. Hofmann and S. Mayboroda, Hardy and BMO spaces associated to divergence form elliptic operators, Math. Ann. 344 (2009), 37-116. MR2481054 (2009m:42038)

[40] S. Hofmann and S. Mayboroda, Correction to "Hardy and BMO spaces associated to divergence form elliptic operators", arXiv: 0907.0129v2.

[41] S. Hofmann, S. Mayboroda and A. McIntosh, Second order elliptic operators with complex bounded measurable coefficients in $L^{p}$, Sobolev and Hardy spaces, Ann. Sci. École Norm. Sup. (4) 44 (2011), 723-800. MR2931518

[42] G. Hu, D. Yang and Y. Zhou, Boundedness of singular integrals in Hardy spaces on spaces of homogeneous type, Taiwanese J. Math. 13 (2009), 91-135. MR2489309 (2010a:42048)

[43] J. Huang, Hardy spaces associated to the Schrödinger operator on strongly Lipschitz domains of $\mathbb{R}^{d}$, Math. Z. 266 (2010), 141-168. MR2670676 (2011e:42046)

[44] J. Huang, A characterization of Hardy space on strongly Lipschitz domains of $\mathbb{R}^{n}$ by Littlewood-Paley-Stein function, Commun. Contemp. Math. 12 (2010), 71-84. MR2649228 (2011c:42034)

[45] T. Iwaniec and J. Onninen, $\mathcal{H}^{1}$-estimates of Jacobians by subdeterminants, Math. Ann. 324 (2002), 341-358. MR.1933861 (2003i:42027)

[46] S. Janson, Generalizations of Lipschitz spaces and an application to Hardy spaces and bounded mean oscillation, Duke Math. J. 47 (1980), 959-982. MR596123 (83j:46037)

[47] D. Jerison and C. Kenig, The inhomogeneous Dirichlet problem in Lipschitz domains, J. Funct. Anal. 130 (1995), 161-219. MR.1331981 (96b:35042)

[48] R. Jiang and D. Yang, Orlicz-Hardy spaces associated with operators satisfying DaviesGaffney estimates, Commun. Contemp. Math. 13 (2011), 331-373. MR2794490 (2012e:42040)

[49] R. Jiang and D. Yang, New Orlicz-Hardy spaces associated with divergence form elliptic operators, J. Funct. Anal. 258 (2010), 1167-1224. MR2565837 (2011e:42047)

[50] R. Jiang, Da. Yang and Do. Yang, Maximal function characterizations of Hardy spaces associated with magnetic Schrödinger operators, Forum Math. 24 (2012), 471-494.

[51] R. Jiang, D. Yang and Y. Zhou, Orlicz-Hardy spaces associated with operators, Sci. China Ser. A 52 (2009), 1042-1080. MR2505009 (2011b:46054)

[52] R. Jiang, D. Yang and Y. Zhou, Localized Hardy spaces associated with operators, Appl. Anal. 88 (2009), 1409-1427. MR2574337(2011b:42071)

[53] R. Johnson and C. J. Neugebauer, Homeomorphisms preserving $A_{p}$, Rev. Mat. Ibero. 3 (1987), 249-273. MR990859 (90d:42013)

[54] J. Kadlec, The regularity of the solution of the Poisson problem in a domain whose boundary is similar to that of a convex domain, Czechoslovak Math. J. 14 (1964), 386-393. MR0170088 (30:329)

[55] T. Kato, Perturbation Theory for Linear Operators, Springer-Verlag, New York, 1966. MR0203473 (34:3324)

[56] K. Kurata, An estimate on the heat kernel of magnetic Schrödinger operators and uniformly elliptic operators with non-negative potentials, J. London Math. Soc. (2) 62 (2000), 885-903. MR.1794292 (2001j:35046)

[57] J.-L. Lions and E. Magenes, Non-homogeneous Boundary Value Problems and Applications, Vol. I, Springer-Verlag, New York-Heidelberg, 1972. MR 0350177 (50:2670)

[58] R. A. Macías and C. Segovia, A decomposition into atoms of distributions on spaces of homogeneous type, Adv. Math. 33 (1979), 271-309. MR546296 (81c:32017b)

[59] S. Martínez and N. Wolanski, A minimum problem with free boundary in Orlicz spaces, Adv. Math. 218 (2008), 1914-1971. MR2431665(2009h:35456)

[60] S. Mayboroda and M. Mitrea, Sharp estimates for Green potentials on non-smooth domains, Math. Res. Lett. 11 (2004), 481-492. MR2092902 (2005i:35059) 
[61] S. Mayboroda and M. Mitrea, The solution of the Chang-Krantz-Stein conjecture, Proceedings of the conference Harmonic Analysis and its Applications at Tokyo (Zempukuji, 2007), pp. 61-154, Tokyo Woman's Christian University, Tokyo, 2007.

[62] A. McIntosh, Operators which have an $H_{\infty}$ functional calculus, Miniconference on operator theory and partial differential equations (North Ryde, 1986), pp. 210-231, Proc. Centre Math. Anal. Austral. Nat. Univ., 14, Austral. Nat. Univ., Canberra, 1986. MR912940 (88k:47019)

[63] D. Mitrea, M. Mitrea and L. Yan, Boundary value problems for the Laplacian in convex and semiconvex domains, J. Funct. Anal. 258 (2010), 2507-2585. MR2593333 (2010m:42049)

[64] D. Mitrea, I. Mitrea, M. Mitrea and L. Yan, Coercive energy estimates for differential forms in semi-convex domains, Commun. Pure Appl. Anal. 9 (2010), 987-1010. MR2610257 (2011i:35052)

[65] A. Miyachi, Maximal functions for distributions on open sets, Hitotsubashi J. Arts Sci. 28 (1987), 45-58. MR.939226 (90k:42038)

[66] A. Miyachi, $H^{p}$ spaces over open subsets of $\mathbb{R}^{n}$, Studia Math. 95 (1990), 205-228. MR:1060724 (91m:42022)

[67] S. Müller, Hardy space methods for nonlinear partial differential equations, Tatra Mt. Math. Publ. 4 (1994), 159-168. MR.1298466 (95f:35191)

[68] W. Orlicz, Über eine gewisse Klasse von Räumen vom Typus B, Bull. Int. Acad. Pol. Ser. A 8 (1932), 207-220.

[69] E. M. Ouhabaz, Analysis of Heat Equations on Domains, Princeton University Press, Princeton, N. J., 2005. MR2124040 (2005m:35001)

[70] M. Rao and Z. Ren, Theory of Orlicz Spaces, Marcel Dekker, Inc., New York, 1991. MR.1113700(92e:46059)

[71] M. Rao and Z. Ren, Applications of Orlicz Spaces, Marcel Dekker, Inc., New York, 2002. MR $1890178(2003 \mathrm{e}: 46041)$

[72] S. Rolewicz, On a certain class of linear metric spaces, Bull. Acad. Polon. Sci. Cl. III. 5 (1957), 471-473. MR0088682(19:562d)

[73] E. Russ, The atomic decomposition for tent spaces on spaces of homogeneous type, CMA/AMSI Research Symposium "Asymptotic Geometric Analysis, Harmonic Analysis, and Related Topics", pp. 125-135, Proc. Centre Math. Appl., 42, Austral. Nat. Univ., Canberra, 2007. MR2328517 (2008m:46066)

[74] V. S. Rychkov, Littlewood-Paley theory and function spaces with $A_{p}^{\text {loc }}$ weights, Math. Nachr. 224 (2001), 145-180. MR.1821243 (2002k:42045)

[75] S. Semmes, A primer on Hardy spaces, and some remarks on a theorem of Evans and Müller, Comm. Partial Differential Equations 19 (1994), 277-319. MR1257006 (94j:46038)

[76] E. M. Stein, Harmonic Analysis: Real-variable Methods, Orthogonality, and Oscillatory Integrals, Princeton University Press, Princeton, N. J., 1993. MR.1232192 (95c:42002)

[77] E. M. Stein and G. Weiss, On the theory of harmonic functions of several variables. I. The theory of $H^{p}$-spaces, Acta Math. 103 (1960), 25-62. MR0121579 (22:12315)

[78] L. Tang, Weighted local Hardy spaces and their applications, Illinois J. Math. (to appear) or arXiv: 1004.5294.

[79] H. Triebel, Theory of Function Spaces, Birkhäuser Verlag, Basel, 1983. MR781540 (86j:46026)

[80] H. Triebel, Theory of Function Spaces II, Birkhäuser Verlag, Basel, 1992. MR.1163193 (93f:46029)

[81] H. Triebel and H. Winkelvoß, Intrinsic atomic characterizations of function spaces on domains, Math. Z. 221 (1996), 647-673. MR.1385173 (97b:46048)

[82] B. E. Viviani, An atomic decomposition of the predual of $\operatorname{BMO}(\rho)$, Rev. Mat. Iberoamericana 3 (1987), 401-425. MR.996824 (90e:46024)

[83] H. Wang and H. Jia, Potential space estimates in local Hardy spaces for Green potentials in convex domains, Anal. Theory Appl. 20 (2004), 342-349. MR2120009

[84] H. Wang and X. Yang, The characterization of the weighted local Hardy spaces on domains and its application, J. Zhejiang Univ. Sci. 9 (2004), 1148-1154.

[85] L. Yan, Classes of Hardy spaces associated with operators, duality theorem and applications, Trans. Amer. Math. Soc. 360 (2008), 4383-4408. MR2395177(2010f:42045)

[86] D. Yang and S. Yang, Weighted local Orlicz-Hardy spaces with applications to pseudodifferential operators, Dissertationes Math. (Rozprawy Mat.) 478 (2011), 1-78. MR2848094 (2012h:46056) 
[87] D. Yang and S. Yang, Real-variable characterizations of Orlicz-Hardy spaces on strongly Lipschitz domains of $\mathbb{R}^{n}$, Rev. Mat. Iberoam. 29 (2013), 237-292.

[88] D. Yang and S. Yang, Orlicz-Hardy spaces associated with divergence operators on unbounded strongly Lipschitz domains of $\mathbb{R}^{n}$, Indiana Univ. Math. J. 61 (2012), 81-129.

[89] K. Yosida, Functional Analysis, Springer-Verlag, Berlin, 1995. MR1336382 (96a:46001)

School of Mathematical Sciences, Beijing Normal University, Laboratory of Mathematics and Complex Systems, Ministry of Education, Beijing 100875, People's Republic OF CHINA

E-mail address: caojun1860@mail.bnu.edu.cn

Department of Mathematics and Department of Computer Science, Georgetown UniVERSity, WASHINGTON, DC 20057

E-mail address: chang@georgetown.edu

School of Mathematical Sciences, Beijing Normal University, Laboratory of Mathematics and Complex Systems, Ministry of Education, Beijing 100875, People's Republic OF CHINA

E-mail address: dcyang@bnu.edu.cn

School of Mathematical Sciences, Beijing Normal University, Laboratory of Mathematics and Complex Systems, Ministry of Education, Beijing 100875, People's Republic OF CHINA

E-mail address: yangsibei@mail.bnu.edu.cn 